\title{
Lógica de topos e aplicações
}

\author{
Arthur Francisco Schwerz Cahali \\ DiSSERTAÇÃO APRESENTADA \\ $\mathrm{AO}$ \\ Instituto DE MATEMÁticA E EstatísticA \\ DA \\ UniversidAde DE SÃo PAUlo \\ PARA \\ OBTENÇÃO DO TÍTULO \\ $\mathrm{DE}$ \\ Mestre em CiÊnCIAS \\ Programa: Matemática \\ Orientador: Hugo Luiz Mariano
}

Durante o desenvolvimento deste trabalho o autor recebeu auxílio financeiro da CAPES

São Paulo, maio de 2019 


\section{Lógica de topos e aplicações}

Esta versão da dissertação contém as correções e alterações sugeridas pela Comissão Julgadora durante a defesa da versão original do trabalho, realizada em 12/06/2019. Uma cópia da versão original está disponível no

Instituto de Matemática e Estatística da Universidade de São Paulo.

Comissão Julgadora:

- Prof. Dr. Hugo Luiz Mariano (orientador) - IME-USP

- Prof. Dr. Marcelo Esteban Coniglio - UNICAMP

- Prof. Dr. Vinícius Cifú Lopes - UFABC 


\section{Agradecimentos}

Primeiramente, gostaria de agradecer meus pais, Cláudia e Francisco, por todo apoio durante essa jornada, e ao meu pai, em particular, por me ajudar com a revisão do texto. Gostaria também de agradecer meus colegas pela amizade e convivência ao longo dos anos: Ana Tenório, Ivo Terek, Luiz Fushimi, Pedro Brack e Rafael Bordoni, dentre outros lembrados mas não aqui nominados.

Faço um agradecimento especial ao meu orientador, Hugo Luiz Mariano, por toda dedicação, cuidado e apoio constante no decorrer do mestrado, sem os quais este trabalho teria sido possível.

Aos professores Marcelo Coniglio e Vinícius Lopes, sou grato pela cuidadosa análise do trabalho e proveitosas sugestões fornecidas.

Por fim, agradeço a CAPES, Coordenação de Aperfeiçoamento de Pessoal de Nível Superior, pelo auxílio financeiro. 


\section{Resumo}

CAHALI, A. F. S. Lógica de topos e aplicações. 2019. 201 f. Dissertação (Mestrado) Instituto de Matemática e Estatística, Universidade de São Paulo, São Paulo, 2019.

A primeira noção de topos, a de topos de Grothendieck, surgiu há cerca de 50 anos a partir de uma generalização do conceito de feixe na geometria algébrica. Poucos anos mais tarde, uma axiomatização categorial de algumas das propriedades de um topos de Grothendieck deu origem a uma segunda noção de topos, a de topos elementar; e essa descrição permitiu estabelecer ligações entre essas categorias e teoria dos conjuntos e lógica. Neste trabalho, estudamos a teoria de topos com um foco especial na construção da lógica interna dos topoi, e exploramos sua relação com modelos Heyting-valorados.

Palavras-chave: topos, lógica categorial, modelos Heyting-valorados. 


\section{Abstract}

CAHAli, A. F. S. Topos Logic and Applications. 2019. 201 f. Dissertação (Mestrado) Instituto de Matemática e Estatística, Universidade de São Paulo, São Paulo, 2019.

The first definition of a topos, that of a Grothendieck topos, emerged roughly 50 years ago from a generalization of the notion of sheaves in algebraic geometry. Few years later, a categorical axiomatization of some properties of Grothendieck topoi gave rise to a second notion of topoi, that of an elementary topos; and this description made it possible to establish connections between these categories and set theory and logic. In this work, we study topos theory with a particular focus on the construction of the internal logic of topoi, and explore its relation to Heyting-valued models.

Keywords: topos, categorical logic, Heyting-valued models. 


\section{Sumário}

Introdução 1

1 Preliminares 5

1.1 Conceitos básicos de teoria das categorias . . . . . . . . . . . . . 6

1.2 Objetos e flechas especiais . . . . . . . . . . . . . . . . . . 13

1.3 Transformações naturais . . . . . . . . . . . . . . . . . . . . . . . 15

1.4 Limites . . . . . . . . . . . . . . . . . . . . . . . . . 19

1.5 Alguns resultados sobre limites . . . . . . . . . . . . . . . . 25

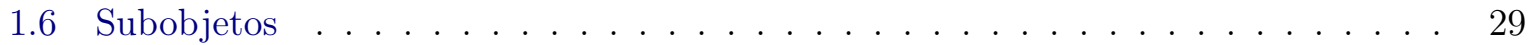

1.7 Adjunção . . . . . . . . . . . . . . . . . . . . . . . . 33

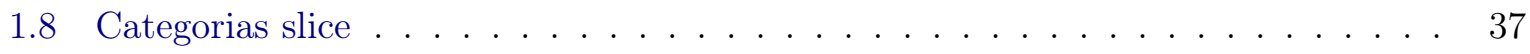

1.9 Objeto números naturais . . . . . . . . . . . . . . . . . . . . . . 41

2 Topos de Grothendieck 43

2.1 Feixes sobre espaços topológicos . . . . . . . . . . . . . . . . . . . . . . . 43

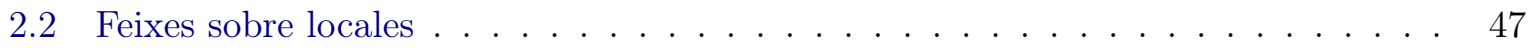

2.3 Feixes sobre sítios e topos de Grothendieck . . . . . . . . . . . . . . . 57

$\begin{array}{llr}3 & \text { Topos elementar } & 67\end{array}$

3.1 Categorias cartesianamente fechadas . . . . . . . . . . . . . . . . . 68

3.2 Classificador de subobjetos . . . . . . . . . . . . . . . . 73

3.3 Topos elementar . . . . . . . . . . . . . . . . . . . . . 77

3.4 Algumas propriedades de topoi . . . . . . . . . . . . . . . . . . . . 81

3.5 Exemplos de topoi . . . . . . . . . . . . . . . . . . . . . . . 91 
3.6 Tipos de topoi $\ldots \ldots \ldots \ldots \ldots$

3.7 Topologias e feixes num topos . . . . . . . . . . . . . . . . . 100

$\begin{array}{lll}4 & \text { Lógica de topos } & 103\end{array}$

4.1 Conjunção . . . . . . . . . . . . . . . . . . . . . . . . . . . . . . . 104

4.2 Disjunção . . . . . . . . . . . . . . . . . . . . 107

4.3 Implicação . . . . . . . . . . . . . . . . . . . . . . . . . . 113

4.4 Negação . . . . . . . . . . . . . . . . . . . . . . . . . . . . . . . 117

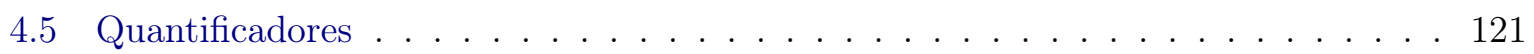

4.6 Linguagem de Mitchell-Bénabou . . . . . . . . . . . . . . . . . . . 123

4.7 Semântica de Kripke-Joyal . . . . . . . . . . . . . . . . . . . . . . . . 137

4.8 Objeto números naturais num topos f . . . . . . . . . . . . . . . 143

$5 \quad$ Modelos Heyting-valorados e topoi locálicos $\quad 147$

5.1 Topos locálico . . . . . . . . . . . . . . . . . . . . . . . . . . . 147

5.2 Modelos Heyting-valorados e IZF . . . . . . . . . . . . . . . . . . . . . . 149

$5.3 V^{(H)}$ e descrições equivalentes de $\mathbf{S h}(H) \ldots \ldots \ldots \ldots \ldots \ldots$

5.4 Forcing, modelos a valores booleanos e topoi booleanos . . . . . . . . . . . . . . 161

5.5 Morfismos induzidos em modelos Heyting-valorados . . . . . . . . . . . . . . . 164

5.6 Considerações finais . . . . . . . . . . . . . . . . . . . . . . 175

$\begin{array}{ll}\text { A Teoria dos reticulados } & 179\end{array}$

A.1 Reticulados . . . . . . . . . . . . . . . . . . . . 179

A.2 Álgebras de Heyting . . . . . . . . . . . . . . . . . . . . . . . . . 181

A.3 Álgebras de Boole . . . . . . . . . . . . . . . . . . . . . 183

$\begin{array}{ll}\text { Bibliografia } & 185\end{array}$

$\begin{array}{ll}\text { Lista de símbolos } & 188\end{array}$

$\begin{array}{lr}\text { Índice remissivo } & 190\end{array}$ 


\section{Introdução}

O conceito de topos foi criado por Grothendieck em 1963 no contexto da geometria algébrica, mais especificamente no estudo de cohomologia de espaços generalizados com coeficientes variando num feixe.

De acordo com Gray ([Gra79a]), a teoria dos feixes surge em 1945 com Leray dentro da topologia algébrica, como ferramenta para o estudo de complexos de cadeias com suporte num espaço topológico. Para ilustrar a ideia, utilizemos o exemplo dado em [LM94]: a grosso modo, um feixe $A$ de grupos abelianos sobre um espaço topológico $X$ é uma família de grupos abelianos $A_{x}$ parametrizada por pontos $x \in X$ de forma "adequadamente contínua". Em particular, isso significa que fazemos da união disjunta $\amalg A_{x}$ um espaço munido de uma topologia tal que o mapa $A_{x} \mapsto x$ é contínuo e étale (ou seja, é "localmente um homeomorfismo").

Dado um aberto $U \subseteq X$, pode-se considerar a seção $s$ do feixe $A$ sobre $U$, que é uma função (novamente "adequadamente contínua") que leva cada $x \in U$ a um elemento $s(x) \in A_{x}$. Assim, dado outro aberto $V \subseteq U$, podemos restringir $s$ a $V$; e reciprocamente, podemos recuperar uma seção $s$ sobre $U$ fazendo a colagem das restrições de $s$ aos $V_{i}$ 's tais que $U=\bigcup V_{i}$. Um feixe $A$, portanto, pode ser descrito apenas com as seções sobre os abertos de $X$, juntamente com as operações de restrição e colagem. Isso possibilitou definir a cohomologia correspondente de um espaço topológico com coeficientes num feixe.

Essa noção foi, então, adaptada à geometria algébrica, onde a construção de um feixe sobre um espaço topológico $X$ poderia ser feita a partir de seções definidas em objetos $U$, não mais necessariamente subconjuntos de $X$, mas simplesmente usando os mapas $U \rightarrow X$ de outro espaço $U$ em $X$. Com isso, ideias da teoria das categorias passaram a ser usadas para o estudo de feixes, levando Grothendieck a definir feixes num contexto mais geral: usando objetos de uma categoria $\mathcal{C}$ nos quais famílias adequadas de flechas $U_{i} \rightarrow X$ formam "coberturas" dos objetos $X$ de $\mathcal{C}$. Tal estrutura, que faz os objetos de $\mathcal{C}$ agirem como abertos de uma topologia, é chamada de topologia 
de Grothendieck. Dessa forma, um feixe tornou-se um funtor, cujos "estágios" são determinados coerentemente por colagem de subestágios sobre cada cobertura. É daí que obtemos nossa primeira noção de topos, o topos de Grothendieck: a categoria dos feixes de conjuntos sobre uma categoria munida de uma topologia de Grothendieck. Essa descrição permitiu importantes avanços na geometria algébrica nos anos seguintes.

Uma segunda abordagem dos topoi nasce mais diretamente da teoria das categorias. Categorias surgiram primeiramente como uma ferramenta no contexto da topologia algébrica, mas na década de 1940, com trabalhos de Eilenberg e Mac Lane, surgiu a teoria das categorias como disciplina abstrata. Como ressaltado por Goldblatt em [Gol79], um aspecto dessa teoria é que o conceito de flechas entre objetos (uma abstração de funções entre conjuntos) pode ser usada em lugar da relação de pertencimento como base para construções matemáticas, e tais flechas podem expressar propriedades de entidades matemáticas.

Lawvere, em 1963, começou a trabalhar em criar uma fundamentação puramente categorial da matemática (diferente da fundamentação conjuntista usual), iniciando com a axiomatização apropriada da categoria dos conjuntos. Ao conhecer propriedades do topos de Grothendieck, notou que tal topos permitia operações básicas da teoria dos conjuntos, como a construção de conjuntos de funções de um conjunto no outro, ou do conjunto das partes de um conjunto. $\mathrm{Na}$ mesma época, Tierney percebeu que o trabalho de Grothendieck poderia levar a um estudo axiomático de feixes. Trabalhando juntos, Lawvere e Tierney criaram, no início dos anos 1970, o conceito abstrato de topos elementar, formulado inteiramente com linguagem categorial, como axiomatização para categorias de feixes de conjuntos (usando o conceito importante - presente nos topoi de Grothendieck - do classificador de subobjeto).

Noções de topos adicionais surgiram posteriormente, algumas inclusive oriundas da ciência da computação teórica. O importante, como enfatiza Johnstone em [Joh02a], é que todas essas noções descrevem aspectos de uma mesma teoria, e mostrar isso é um dos objetivos de seu livro de três volumes. No presente projeto, focaremos o estudo mais especificamente no desenvolvimento da lógica no contexto dos topoi de Grothendieck e dos topoi elementares.

$\mathrm{Na}$ categoria dos conjuntos, as relações lógicas são representadas pelas operações de uma álgebra de Boole com dois elementos. O topos elementar, por sua vez, possui uma álgebra (agora uma álgebra de Heyting) que pode cumprir o mesmo papel de representar princípios lógicos; isso permite considerar, portanto, uma lógica interna ao topos. Uma diferença relevante é que num topos, a lógica não é mais, em geral, clássica, mas sim intuicionista. 
Aplicações interessantes em outras áreas da matemática surgiram dessa internalização da lógica num topos como uma maneira de sintetizar as relações entre objetos externamente constituídos (como feixes, por exemplo), ocorrendo primeiro em áreas como teoria dos conjuntos (provas de independência de axiomas), álgebra e geometria (como modelos para a geometria diferencial sintética), e mais recentemente em análise, aritmética e até em física quântica. Aqui exploraremos uma aplicação de forma um pouco mais detalhada: a relação da lógica de topos com modelos Heyting-valorados, que são (como o nome sugere) modelos em que os valores verdade estão numa álgebra de Heyting completa.

A motivação para escolha do tema, aliás, veio do estudo, durante a iniciação científica, da teoria algébrica dos conjuntos. Criada na década de 1990 por Joyal e Moerdijk, a teoria algébrica dos conjuntos é uma nova abordagem para a construção de modelos de teorias de conjuntos, onde os modelos para teorias de conjuntos são tratados como álgebras para uma teoria algébrica adequada. Os topoi foram inspiração para a criação de tal teoria, e são ferramentas importantes na sua construção.

Em relação à estrutura da dissertação, o primeiro capítulo é dedicado a uma introdução à teoria das categorias, dado que é com a linguagem de categorias que os topoi são definidos. Nele apresentamos as definições iniciais (categorias, funtores, transformações naturais) e construções importantes para o estudo dos topoi (como limites e adjunções) da teoria, além de registrar alguns resultados.

O objetivo do segundo capítulo é apresentar os topoi de Grothendieck. Primeiramente, descrevemos as duas noções equivalentes de feixes sobre espaços topológicos: feixes geométricos e feixes funtoriais. Em seguida, fazemos o mesmo para feixes sobre locales, indicando algumas das adaptações necessárias, e construímos os funtores que relacionam a categoria dos espaços topológicos com a categorias dos locales. Finalmente, generalizamos o conceito de feixes para um contexto ainda mais geral, o dos feixes sobre sítios (categorias munidas de uma noção de cobertura adequada); com isso, um topos de Grothendieck será definido como uma categoria equivalente à categoria dos feixes sobre um sítio.

Iniciamos o terceiro capítulo introduzindo categorias cartesianamente fechadas e classificadores de subobjetos, e utilizando esses dois conceitos definimos topoi elementares. Mostramos, também, uma forma equivalente de definir tais topoi, usando a noção de objeto partes, e então apresentamos alguns dos principais resultados (como o teorema fundamental e a fatoração epimono) e exemplos da teoria de topos. Para finalizar, registramos brevemente alguns tipos de 
topoi, e mostramos como definir topologias e feixes num topos.

Na primeira parte do quarto capítulo, dedicamo-nos a definir operações no conjunto dos subobjetos de um objeto num topos e mostrar que, com elas, os subobjetos adquirem uma estrutura de álgebra de Heyting. Esse resultado será importante para, na segunda parte do capítulo, introduzirmos uma linguagem para a lógica interna de um topos, com uma semântica correspondente.

Finalmente, no quinto capítulo investigamos a relação dos topoi com modelos a valores numa álgebra de Heyting. Primeiro definimos topoi locálicos e o modelo Heyting-valorado $V^{(H)}$, e mostramos como podemos obter equivalências de categorias que conectam ambas as noções. Em seguida, exploramos a relação entre forcing (da teoria dos conjuntos), a semântica de modelos a valores booleanos e topoi booleanos; e encerramos o capítulo estudando morfismos induzidos em modelos Heyting-valorados a partir de morfismos de álgebras de Heyting (algo que não foi encontrado na literatura).

Concluído o corpo principal do texto, como em seu decorrer serão usadas algumas noções e resultados da teoria de reticulados, incluímos um breve apêndice para registrar algumas definições e propriedades iniciais da teoria, com um foco em álgebras de Heyting e de Boole. 


\section{Capítulo 1}

\section{Preliminares}

Este capítulo visa introduzir, sucintamente, definições e propriedades de teorias das categorias que serão usadas nos capítulos posteriores. Primeiro definimos os conceitos básicos de categoria, funtor e transformação natural, além de apresentarmos algumas noções derivadas (como categorias produto e funtores hom) e alguns resultados importantes (como o Lema de Yoneda). Então, passamos a descrever e indicar propriedades de diversas construções categoriais relevantes para o estudo de topos: limites, subobjetos, adjunção, categorias slice e objetos números naturais.

Para não alongar o texto desnecessariamente, várias demonstrações e verificações serão omitidas; porém, algumas serão feitas (ou, no mínimo, esboçadas) para ilustrar argumentos que serão usados posteriormente (por exemplo na seção que trata de subobjetos).

Para uma apresentação mais completa de teoria das categorias, pode-se consultar [Bor08a] ou [Lan98]. É comum livros de teoria de topos também dedicarem capítulos iniciais a categorias, como fazem [BW85], [Bel88], [Gol79], [LM94] e [Mc195]. Outra referência interessante é o site nLab (https://ncatlab.org/), um wiki que serve como uma espécie de enciclopédia especializada em teorias das categorias ${ }^{1}$, sendo útil inclusive para buscar referências bibliográficas. ${ }^{2}$

\footnotetext{
${ }^{1} \mathrm{O}$ site é mantido pelo matemático Urs Schreiber, mas sendo um wiki possui contribuições de diversos autores (a lista completa deles pode ser consultada em https://ncatlab.org/nlab/authors).

${ }^{2}$ Aliás, diversas páginas (em inglês) da Wikipedia (https://en.wikipedia.org/) sobre categorias e topoi, além de serem úteis por listar bibliografia, são por si só razoavelmente bem escritas. Embora frequentemente não forneçam detalhes técnicos, podem ser boas para uma primeira introdução aos conceitos.
} 


\subsection{Conceitos básicos de teoria das categorias}

Antes de começar, há de ser feita uma breve observação em relação a questões de fundamentação matemática: a teoria das categorias trabalha frequentemente com objetos "grandes", isto é, que não são conjuntos (por exemplo, com a "coleção" dos conjuntos ou com a "coleção" dos espaços topológicos). Isso traz complicações para formalizar conceitos de teoria das categorias dentro de teoria dos conjuntos.

Para lidar com essa dificuldade, pode-se usar uma teoria como NBG. Nela, a noção primitiva é a de "classe". Nessa teoria, os conjuntos são as classes que pertencem a alguma outra classe; e uma classe que não é um conjunto é chamada de uma classe própria. Outra possibilidade seria usar "universos", um conjunto dentro do qual pode-se realizar as operações usuais de teoria dos conjuntos, e assumir que todo conjunto pertence a um universo (esse conceito pode ser formalizado através dos universos de Grothendieck ${ }^{3}$ ).

O foco aqui não é discutir e detalhar tais formalizações, apenas ter em mente que elas nos fornecem maneiras formais de tratar desses objetos "grandes" evitando complicações conjuntoteóricas.

Definição 1.1.1. Uma categoria $\mathcal{C}=\left(\mathcal{C}_{0}, \mathcal{C}_{1}\right.$, dom, $\left.\operatorname{cod}, u, k\right)$ consiste de:

- uma classe $\mathcal{C}_{0}$ de objetos;

- uma classe $\mathcal{C}_{1}$ de flechas;

- uma função domínio dom : $\mathcal{C}_{1} \rightarrow \mathcal{C}_{0}$ e uma função codomínio cod : $\mathcal{C}_{1} \rightarrow \mathcal{C}_{0}$. Quando $\operatorname{dom}(f)=a$ e $\operatorname{cod}(f)=b$, para $f \in \mathcal{C}_{1}$, denotaremos $f: a \rightarrow b$ ou $a \stackrel{f}{\rightarrow} b ;$

- uma função identidade $u: \mathcal{C}_{0} \rightarrow \mathcal{C}_{1}$, com $\operatorname{dom}\left((u(a))=\operatorname{cod}(u(a))=a\right.$, para cada $a \in \mathcal{C}_{0}$. Denotaremos $u(a):=i d_{a}$;

- uma função composição $k:\left\{(f, g) \in \mathcal{C}_{1} \times \mathcal{C}_{1} \mid \operatorname{dom}(g)=\operatorname{cod}(f)\right\} \rightarrow \mathcal{C}_{1}, \operatorname{com} \operatorname{dom}(k(f, g))=$ $\operatorname{dom}(f)$ e $\operatorname{cod}(k(f, g))=\operatorname{cod}(g)$, para cada $f, g \in \mathcal{C}_{1}$. Denotaremos $k(f, g):=g \circ f$ e $\operatorname{comp}(\mathcal{C}):=\left\{(f, g) \in \mathcal{C}_{1} \times \mathcal{C}_{1} \mid \operatorname{dom}(g)=\operatorname{cod}(f)\right\}$.

Essas funções devem satisfazer:

\footnotetext{
${ }^{3}$ A formulação dos universos de Grothendieck foi a originalmente utilizada por Grothendieck para introduzir a teoria dos topoi de Grothendieck; em especial, o apêndice de Bourbaki ao primeiro capítulo de [AGV72] explora esse tópico.
} 
- Associatividade: para quaisquer $a, b, c, d \in \mathcal{C}_{0}$ e $f: a \rightarrow b, g: b \rightarrow c, h: c \rightarrow d$ em $\mathcal{C}_{1}$, temos $h \circ(g \circ f)=(h \circ g) \circ f$. Pode-se representar isso através do seguinte diagrama comutativo ${ }^{4}$ :

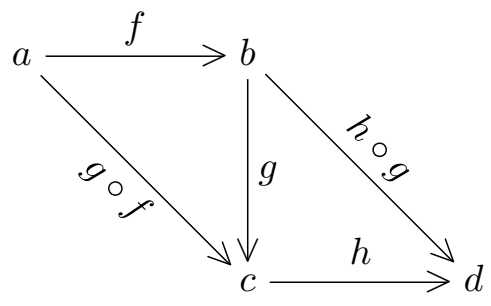

- Unidade: para quaisquer $a, b \in \mathcal{C}_{0}$ e $f: a \rightarrow b$ em $\mathcal{C}_{1}$, temos $i d_{b} \circ f=f$ e $f \circ i d_{a}=f$. Pode-se representar isso através do seguinte diagrama comutativo:

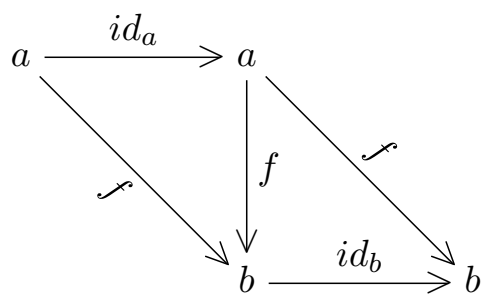

Exemplo 1.1.2. Alguns exemplos iniciais de categorias:

- Set: os objetos são os conjuntos, as flechas são as funções entre eles;

- Top: os objetos são os espaços topológicos, as flechas são as funções contínuas;

- Grp: os objetos são os grupos, as flechas são os homomorfismos de grupos.

Apenas as classes de objetos e flechas foram especificadas, mas o restante da estrutura de categoria pode ser facilmente inferido (por exemplo, a composição em Set é simplesmente a composição usual de funções).

Dada uma categoria $\mathcal{C}$, a classe das flechas entre dois objetos $a, b \in \mathcal{C}_{0}$ será denotada por $\mathcal{C}(a, b):=\left\{f \in \mathcal{C}_{1} \mid \operatorname{dom}(f)=a, \operatorname{cod}(f)=b\right\}$ (outra notação comum é $H_{o} m_{\mathcal{C}}(a, b)$ ).

Exemplo 1.1.3. Seja $(P, \leq)$ um conjunto preordenado, isto é, em que a relação $\leq$ é reflexiva e transitiva. Podemos vê-lo como uma categoria $\mathcal{C}$ fazendo $\mathcal{C}_{0}=P$ e, para cada $p, q \in P$ :

$$
\mathcal{C}(p, q)= \begin{cases}\{*\} & , \text { se } p \leq q \\ \emptyset & , \text { caso contrário }\end{cases}
$$

\footnotetext{
${ }^{4}$ Os diagramas comutativos serão tratados de forma "ingênua" como diagramas nos quais "todos os caminhos (por composição) com mesmos vértices inicial e final são iguais" (com exceção de flechas paralelas). Seria possível dar uma definição precisa (por exemplo, como feito em [Gro57]), mas não será necessário pois aqui serão usados mais de forma ilustrativa.
} 
onde $\{*\}$ denota qualquer conjunto de um elemento (essa notação para um conjunto unitário será usada frequentemente no decorrer do texto). A transitividade de $\leq$ nos permite definir a composição com as propriedades necessárias. A reflexividade garante a existência das identidades.

Definição 1.1.4. Sejam $\mathcal{C}$ uma categoria e $i: a \rightarrow b$ em $\mathcal{C}_{1}$. Dizemos que a flecha $i$ é um isomorfismo (ou apenas um iso) se existe $h: b \rightarrow a \mathrm{em} \mathcal{C}_{1}$ tal que $i \circ h=i d_{b}$ e $h \circ i=i d_{a}$. Quando existe um iso entre dois objetos $a$ e $b$, dizemos que esses objetos são isomorfos, e denotamos $a \cong b$.

De maneira menos formal, para economizar na redação, dizemos que um objeto $a$ com uma propriedade é "único a menos de isomorfismo" se, para todo objeto $b$ com essa propriedade, $a \cong b .^{5}$

Exemplo 1.1.5. Em Set, isos correspondem às funções bijetoras. Em Top, isos correspondem aos homeomorfismos.

Proposição 1.1.6. Sejam $\mathcal{C}$ uma categoria $e(i, j) \in \operatorname{comp}(\mathcal{C})$ um par de isos. Então:

1. existe uma única flecha $h \in \mathcal{C}_{1}$ tal que $i \circ h$ e $h \circ i$ são as identidades. Assim, podemos denotar $h=i^{-1}$;

2. $i^{-1}$ também é iso;

3. $j \circ i$ é iso $e(j \circ i)^{-1}=i^{-1} \circ j^{-1}$;

4. para todo $a \in \mathcal{C}_{0}, i d_{a}$ é iso.

Definição 1.1.7. Uma categoria $\mathcal{C}$ é dita pequena se $\mathcal{C}_{1}$ é conjunto (isso já implica em $\mathcal{C}_{0}$ também o ser). Uma categoria $\mathcal{C}$ é dita localmente pequena se, para todos $a, b \in \mathcal{C}_{0}, \mathcal{C}(a, b)$ é conjunto.

Definição 1.1.8. Sejam $\mathcal{A}, \mathcal{B}$ categorias. Um funtor $F: \mathcal{A} \rightarrow \mathcal{B}$ é um par de funções $F=$ $\left(F_{0}, F_{1}\right)$, onde $F_{0}: \mathcal{A}_{0} \rightarrow \mathcal{B}_{0}$ e $F_{1}: \mathcal{A}_{1} \rightarrow \mathcal{B}_{1}$ são tais que, para toda $f: a \rightarrow a^{\prime}$ em $\mathcal{A}_{1}$, $F_{1}(f): F_{0}(a) \rightarrow F_{0}\left(a^{\prime}\right)$; e vale:

$$
F_{1}\left(i d_{a}\right)=i d_{F_{0}(a)}, \text { para todo } a \in \mathcal{A}_{0}
$$

$$
F_{1}(g \circ f)=F_{1}(g) \circ F_{1}(f), \text { para todos } f, g \in \operatorname{comp}(\mathcal{A})
$$

\footnotetext{
${ }^{5}$ Ao estudar estruturas matemáticas (como grupos, espaços topológicos, etc.), é comum considerar a unicidade a menos de isomorfismo em vez da unicidade estrita (com igualdade). Isso porque, dada uma estrutura, é fácil definir uma "cópia" idêntica mas não exatamente igual a ela. Por exemplo, dado o anel dos inteiros $\mathbb{Z}=\{\ldots,-1,0,1, \ldots\}$, podemos considerar um anel $\mathbb{Z}^{\prime}=\left\{\ldots,-1^{\prime}, 0^{\prime}, 1^{\prime}, \ldots\right\}$ definindo as operações exatamente como nos inteiros; esse novo anel será idêntico aos inteiros do ponto de vista da teoria dos anéis, mas não igual pois o conjunto subjacente é diferente. A unicidade a menos de isomorfismo em categorias (onde agora estruturas são objetos de uma categoria) tenta capturar essa ideia: isos definem uma relação de equivalência entre os objetos.
} 
Assim, a ideia é que um funtor seja um "morfismo de categorias", no sentido de preservar a estrutura algébrica que define categorias.

\section{Exemplo 1.1.9.}

- Um exemplo simples é o funtor partes $\mathcal{P}$ : Set $\rightarrow$ Set, que leva cada conjunto $X$ ao seu conjunto de subconjuntos $\mathcal{P}_{0}(X):=\mathcal{P}(X)$, e cada função $f: X \rightarrow Y$ à função imagem direta $\mathcal{P}_{1}(f): \mathcal{P}(X) \rightarrow \mathcal{P}(Y)$ : a função tal que $\mathcal{P}_{1}(f)(S):=f(S)$, para todo $S \subseteq X$;

- um "tipo" de funtor que "esquece" parte da estrutura algébrica de uma categoria é chamado de um funtor esquecimento. Por exemplo, temos $U: \mathbf{A b} \rightarrow$ Set o funtor que leva cada grupo abeliano ao seu conjunto subjacente, e cada homomorfismo a si mesmo, agora entendido apenas como uma função;

- há também exemplos importantes que surgem em outras áreas da matemática, como na topologia algébrica: o mapa $X \mapsto H_{n}(X)$, que leva cada espaço topológico $X$ ao seu $n$ ésimo grupo de homologia (e cada função contínua a seu homomorfismo correspondente), define um funtor $\mathbf{T o p} \rightarrow \mathbf{A b}$;

- para um exemplo na álgebra, seja $n \in \mathbb{N}$ e considere, para cada anel comutativo $R$, $G L_{n}(R)$ o conjunto das matrizes $n \times n$ invertíveis com entradas em $R$ (também chamado de grupo linear geral). Para cada homomorfismo de aneis comutativos $f: R \rightarrow R^{\prime}$, defina $\left(G L_{n}\right)_{1}(f): G L_{n}(R) \rightarrow G L_{n}\left(R^{\prime}\right)$ como "aplicar $f$ a cada coordenada da matriz". Então, $G L_{n}: \mathbf{c R n g} \rightarrow$ Grp define um funtor.

Definição 1.1.10. Sejam $\mathcal{A}, \mathcal{B}, \mathcal{C}$ categorias e $F: \mathcal{A} \rightarrow \mathcal{B}, G: \mathcal{B} \rightarrow \mathcal{C}$ funtores. Definimos sua composição $G \circ F: \mathcal{A} \rightarrow \mathcal{C}$ fazendo

$$
\begin{aligned}
& (G \circ F)_{0}:=G_{0} \circ F_{0} \\
& (G \circ F)_{1}:=G_{1} \circ F_{1}
\end{aligned}
$$

O funtor identidade $i d_{\mathcal{A}}: \mathcal{A} \rightarrow \mathcal{A}$ é definido como a identidade pela composição de funtores.

Com isso definido, pode-se mostrar que as categorias pequenas e os funtores entre elas formam uma categoria Cat, a categoria das categorias pequenas. Note que Cat não é uma categoria pequena, portanto Cat $\notin \mathbf{C a t}$, e não temos aqui um problema com uma forma do "paradoxo de Russell".

Definição 1.1.11. Sejam $\mathcal{A}, \mathcal{B}$ categorias e $F: \mathcal{A} \rightarrow \mathcal{B}$ um funtor. Dados $a, a^{\prime} \in \mathcal{A}_{0}$, considere o mapa $\phi_{a, a^{\prime}}: \mathcal{A}\left(a, a^{\prime}\right) \rightarrow \mathcal{B}\left(F_{0}(a), F_{0}\left(a^{\prime}\right)\right)$ dado por $\phi_{a, a^{\prime}}(f)=F_{1}(f)$. Com isso: 
1. F é dito pleno se $\phi_{a, a^{\prime}}$ for sobrejetor, para todos $a, a^{\prime} \in \mathcal{A}_{0}$;

2. $F$ é dito fiel se $\phi_{a, a^{\prime}}$ for injetor, para todos $a, a^{\prime} \in \mathcal{A}_{0}$;

3. F é dito um isomorfismo de categorias se for pleno, fiel e $F_{0}$ for uma bijeção (equivalentemente, será isomorfismo se existir um funtor $G: \mathcal{B} \rightarrow \mathcal{A}$ tal que $G \circ F=i d_{\mathcal{A}}$ e $\left.F \circ G=i d_{\mathcal{B}}\right)$.

Semelhante ao caso para objetos, se existe um isomorfismo entre duas categorias $\mathcal{A}$ e $\mathcal{B}$, diremos que essas categorias são isomorfas, e denotaremos $\mathcal{A} \cong \mathcal{B}$. A existência de um isomorfismo de categorias, no entanto, é uma condição muito forte, que é raramente satisfeita na prática. Mais tarde, será introduzida a noção mais relevante de uma equivalência de categorias, em que será exigido que o isomorfismo $F$ tenha um inverso "a menos de isomorfismo de funtores".

Outrossim, dado um funtor $F: \mathcal{A} \rightarrow \mathcal{B}$, observe que $F$ ser pleno não implica as funções $F_{0}$ e $F_{1}$ serem sobrejetoras e, similarmente, $F$ ser fiel não implica as funções $F_{0}$ e $F_{1}$ serem injetoras. Porém, vale que se $F$ for pleno e fiel, então $F_{0}$ é "injetor a menos de isomorfismo" (o nome técnico para isso é que $F$ reflete isomorfismos). Significa dizer que, dados $a, a^{\prime} \in \mathcal{A}_{0}, F_{0}(a) \cong F_{0}\left(a^{\prime}\right)$ implica em $a \cong a^{\prime}$.

Definição 1.1.12. Seja $\mathcal{C}=\left(\mathcal{C}_{0}, \mathcal{C}_{1}\right.$, dom, cod, u,k) uma categoria. Uma subcategoria $\mathcal{C}^{\prime}$ de $\mathcal{C}$ é uma categoria $\mathcal{C}^{\prime}=\left(\mathcal{C}_{0}^{\prime}, \mathcal{C}_{1}^{\prime}, \operatorname{dom}^{\prime}, \operatorname{cod}^{\prime}, u^{\prime}, k^{\prime}\right)$ tal que

$$
\begin{array}{cc}
\mathcal{C}_{0}^{\prime} \subseteq \mathcal{C}_{0} & \mathcal{C}_{1}^{\prime} \subseteq \mathcal{C}_{1} \\
\operatorname{dom}^{\prime}=\operatorname{dom}_{\mid \mathcal{C}_{1}^{\prime}} & \operatorname{cod}^{\prime}=\operatorname{cod}_{\mid \mathcal{C}_{1}^{\prime}} \\
u^{\prime}=u_{\mid \mathcal{C}_{0}^{\prime}} & k^{\prime}=k_{\mid \operatorname{comp}\left(\mathcal{C}^{\prime}\right)}
\end{array}
$$

Denotaremos $\mathcal{C}^{\prime} \subseteq \mathcal{C}$. As funções $i_{0}: \mathcal{C}_{0}^{\prime} \rightarrow \mathcal{C}_{0}$ e $i_{1}: \mathcal{C}_{1}^{\prime} \rightarrow \mathcal{C}_{1}$ tais que

$$
\begin{aligned}
& i_{0}(a)=a, \text { para todo } a \in \mathcal{C}_{0}^{\prime} \\
& i_{1}(f)=f, \text { para todo } f \in \mathcal{C}_{1}^{\prime}
\end{aligned}
$$

formam um funtor $i=\left(i_{0}, i_{1}\right): \mathcal{C}^{\prime} \rightarrow \mathcal{C}$, chamado de funtor inclusão.

Definição 1.1.13. Sejam $\mathcal{C}$ uma categoria e $\mathcal{C}^{\prime} \subseteq \mathcal{C}$ uma subcategoria. $\mathcal{C}^{\prime}$ é dita uma subcategoria plena se o funtor inclusão $i: \mathcal{C}^{\prime} \rightarrow \mathcal{C}$ for pleno.

Definição 1.1.14. Seja $\mathcal{C}=\left(\mathcal{C}_{0}, \mathcal{C}_{1}, d o m, \operatorname{cod}, u, k\right)$ uma categoria. A categoria oposta a $\mathcal{C}$, denotada $\mathcal{C}^{o p}=\left(\mathcal{C}_{0}^{o p}, \mathcal{C}_{1}^{o p}, d_{o m}^{o p}, \operatorname{cod}^{o p}, u^{o p}, k^{o p}\right)$, é obtida fazendo-se 


$$
\begin{gathered}
\mathcal{C}_{0}^{o p}:=\mathcal{C}_{0} \quad \mathcal{C}_{1}^{o p}:=\mathcal{C}_{1} \\
\operatorname{dom}^{o p}:=\operatorname{cod} \quad \operatorname{cod}^{o p}:=\operatorname{dom} \quad u^{o p}:=u \\
k^{o p}(f, g):=k(g, f), \text { para todo }(f, g) \in \operatorname{comp}\left(\mathcal{C}^{o p}\right)
\end{gathered}
$$

Essencialmente, inverte-se o sentido das flechas, e para todos $a, b \in \mathcal{C}_{0}$,

$$
\mathcal{C}(a, b)=\mathcal{C}^{o p}(b, a)
$$

Quando $f: a \rightarrow b$ é uma flecha em $\mathcal{C}_{1}$, denotaremos por $f^{o p}: b \rightarrow a$ a flecha em $\mathcal{C}_{1}^{o p}$ correspondente.

Definição 1.1.15. Sejam $\mathcal{A}, \mathcal{B}$ categorias e $F: \mathcal{A} \rightarrow \mathcal{B}$ um funtor. Definimos o funtor oposto $F^{o p}: \mathcal{A}^{o p} \rightarrow \mathcal{B}^{o p}$ tomando:

$$
\begin{gathered}
F_{0}^{o p}(a)=F_{0}(a), \text { para todo } a \in \mathcal{A}_{0} \\
F_{1}^{o p}\left(f^{o p}\right)=F_{1}(f), \text { para toda } f \in \mathcal{A}_{1}
\end{gathered}
$$

Observação. Alguns autores chamam funtores da forma $F: \mathcal{A}^{o p} \rightarrow \mathcal{B}$ e $G: \mathcal{A} \rightarrow \mathcal{B}^{o p}$ de funtores contravariantes. Isso porque, se compreendidos como "funtores" $F, G: \mathcal{A} \rightarrow \mathcal{B}, F$ e $G$ invertem o sentido das flechas e trocam a ordem da composição.

Exemplo 1.1.16. Um exemplo simples, e semelhante ao funtor partes do exemplo 1.1.9, é o funtor imagem inversa $\mathcal{P}^{*}:$ Set $\rightarrow$ Set, que mais uma vez leva cada conjunto $X$ ao seu conjunto de subconjuntos $\mathcal{P}_{0}^{*}(A):=\mathcal{P}(X)$, e cada função $F: X \rightarrow Y$ à função imagem inversa $\mathcal{P}_{1}^{*}(f): \mathcal{P}(Y) \rightarrow \mathcal{P}(X):$ a função tal que $\mathcal{P}_{1}^{*}(f):=f^{-1}(S)$, para todo $S \subseteq Y$.

Mais adiante, trataremos de outras construções (e propriedades) em teoria das categorias que terão uma "noção dual": uma noção obtida invertendo-se a direção de todas as flechas e a ordem das composições na construção (ou propriedade). O princípio da dualidade (um metateorema na linguagem da teoria das categorias) garante que as construções duais também "façam sentido", isto é, também forneçam construções em teoria das categorias. Esse princípio também garante que se alguma propriedade (que dependa apenas dos axiomas elementares da teoria) é válida, então sua dual também é. ${ }^{6}$

Exemplo 1.1.17. Um tipo de funtor que aparecerá frequentemente no futuro é o chamado funtor hom. Sejam $\mathcal{C}$ uma categoria localmente pequena e $c \in \mathcal{C}_{0}$. Pode-se definir o funtor

\footnotetext{
${ }^{6}$ Para uma formulação mais precisa, recomenda-se consultar a seção II.1 de [Lan98]
} 
$\mathcal{C}(c,-): \mathcal{C} \rightarrow$ Set como a notação sugere:

$$
(\mathcal{C}(c,-))_{0}\left(c^{\prime}\right):=\mathcal{C}\left(c, c^{\prime}\right), \text { para todo } c^{\prime} \in \mathcal{C}_{0}
$$

$$
\begin{aligned}
(\mathcal{C}(c,-))_{1}(f):=\mathcal{C}(c, f): \mathcal{C}(c, a) \rightarrow \mathcal{C}(c, b), & \text { para toda } f: a \rightarrow b \text { em } \mathcal{C}_{1}, \\
& \text { onde } \mathcal{C}(c, f)(g):=f \circ g, \text { para toda } g \in \mathcal{C}(c, a)
\end{aligned}
$$

Similarmente, o funtor hom contravariante $\mathcal{C}(-, c): \mathcal{C}^{o p} \rightarrow$ Set pode ser definido fazendo-se:

$$
(\mathcal{C}(-, c))_{0}\left(c^{\prime}\right):=\mathcal{C}\left(c^{\prime}, c\right), \text { para todo } c^{\prime} \in \mathcal{C}_{0}
$$

$(\mathcal{C}(-, c))_{1}(f):=\mathcal{C}(f, c): \mathcal{C}(b, c) \rightarrow \mathcal{C}(a, c)$, para toda $f: a \rightarrow b$ em $\mathcal{C}_{1}$

$$
\text { onde } \mathcal{C}(f, c)(g):=g \circ f \text {, para toda } g \in \mathcal{C}(b, c)
$$

Além disso, dadas categorias $\mathcal{A}, \mathcal{B}$, um funtor $F: \mathcal{A} \rightarrow \mathcal{C}$ e um funtor $G: \mathcal{B} \rightarrow \mathcal{C}^{o p}$, podemos definir as composições:

$$
\begin{aligned}
& \mathcal{C}(c, F(-)):=\mathcal{C}(c,-) \circ F: \mathcal{A} \rightarrow \text { Set } \\
& \mathcal{C}(G(-), c):=\mathcal{C}(-, c) \circ G: \mathcal{B} \rightarrow \text { Set }
\end{aligned}
$$

Definição 1.1.18. Sejam $\mathcal{A}, \mathcal{B}$ categorias. A categoria produto (binário) de $\mathcal{A}$ por $\mathcal{B}$, denotada por $\mathcal{A} \times \mathcal{B}$, é definida tomando $(\mathcal{A} \times \mathcal{B})_{0}=\mathcal{A}_{0} \times \mathcal{B}_{0},(\mathcal{A} \times \mathcal{B})_{1}=\mathcal{A}_{1} \times \mathcal{B}_{1}$, e definindo as funções domínio, codomínio, identidade e composição componente a componente. ${ }^{7}$

Definição 1.1.19. Sejam $\mathcal{A}, \mathcal{B}, \mathcal{C}, \mathcal{D}$ categorias e $F: \mathcal{A} \rightarrow \mathcal{B}, G: \mathcal{C} \rightarrow \mathcal{D}$ funtores. Definimos o funtor produto $F \times G: \mathcal{A} \times \mathcal{C} \rightarrow \mathcal{B} \times \mathcal{D}$ fazendo:

$$
\begin{aligned}
& (F \times G)_{0}(a, c)=\left(F_{0}(a), G_{0}(b)\right), \text { para todos }(a, c) \in(\mathcal{A} \times \mathcal{C})_{0} \\
& (F \times G)_{0}(f, g)=\left(F_{1}(f), G_{1}(g)\right), \text { para todos }(f, g) \in(\mathcal{A} \times \mathcal{C})_{1}
\end{aligned}
$$

É comum chamar funtores que tenham como domínio uma categoria produto (binário) de um bifuntor.

Exemplo 1.1.20. Com isso, podemos definir o bifuntor hom $\mathcal{C}(-,-): \mathcal{C}^{o p} \times \mathcal{C} \rightarrow$ Set, para $\mathcal{C}$

\footnotetext{
${ }^{7}$ Produtos arbitrários de categorias usam a noção de produto como limite, que será definido mais adiante.
} 
uma categoria localmente pequena. Como a notação sugere:

$$
(\mathcal{C}(-,-))_{0}(a, b):=\mathcal{C}(a, b), \text { para todos } a, b \in \mathcal{C}_{0}
$$

$$
\begin{aligned}
(\mathcal{C}(-,-))_{1}\left(f^{o p}, g\right):=\mathcal{C}\left(f^{o p}, g\right): \mathcal{C}(b, c) & \rightarrow \mathcal{C}(a, d), \text { para todas } f: a \rightarrow b, g: c \rightarrow d \text { em } \mathcal{C}_{1}, \\
& \text { onde } \mathcal{C}\left(f^{o p}, g\right)(h):=g \circ h \circ f, \text { para toda } h \in \mathcal{C}(b, c)
\end{aligned}
$$

Além disso, dadas $\mathcal{A}, \mathcal{B}$ categorias e $F: \mathcal{A} \rightarrow \mathcal{C}$ e $G: \mathcal{B} \rightarrow \mathcal{C}$ funtores, podemos definir as composições:

$$
\begin{gathered}
\mathcal{C}(F(-),-):=\mathcal{C}(-,-) \circ\left(F^{o p} \times i d_{\mathcal{C}}\right): \mathcal{A}^{o p} \times \mathcal{C} \rightarrow \text { Set } \\
\mathcal{C}(-, G(-)):=\mathcal{C}(-,-) \circ\left(i d_{\mathcal{C}^{o p}} \times G\right): \mathcal{C}^{o p} \times \mathcal{B} \rightarrow \text { Set } \\
\mathcal{C}(F(-), G(-)):=\mathcal{C}(-,-) \circ\left(F^{o p} \times G\right): \mathcal{A}^{o p} \times \mathcal{B} \rightarrow \text { set }
\end{gathered}
$$

\subsection{Objetos e flechas especiais}

Definição 1.2.1. Sejam $\mathcal{C}$ uma categoria e $m, e \in \mathcal{C}_{1}$. A flecha $m: a \rightarrow b$ é dita um mono (ou monomorfismo) se, para todas $f, g \in \mathcal{C}_{1} \operatorname{com} f, g: c \rightarrow a, m \circ f=m \circ g$ implica em $f=g$. A flecha $e: a \rightarrow b$ é dita um epi (ou epimorfismo) se, para todas $f, g \in \mathcal{C}_{1} \operatorname{com} f, g: b \rightarrow c$, $f \circ e=g \circ e$ implica em $f=g$.

É comum denotar um mono $m$ por $m: a \longmapsto b$ e um epi $e$ por $e: a \rightarrow b$.

Exemplo 1.2.2. Em Set e em diversas outras categorias (como Top, Grp, Rng, etc.), monos correspondem às funções injetoras (porém existem categorias onde isso não vale, por exemplo na categoria dos grupos abelianos divisíveis e seus homomorfismos). Em Set e em algumas outras categorias (como Top e Grp), epis correspondem às funções sobrejetoras (isso não vale, por exemplo, em Ring).

Se uma flecha é um iso, também é um mono e um epi. Porém, a recíproca nem sempre é verdadeira, como ilustrado pelo exemplo em Top (funções contínuas bijetoras podem não ser homeomorfismos).

Proposição 1.2.3. Sejam $\mathcal{C}$ uma categoria $e(f, g) \in \operatorname{comp}(\mathcal{C})$. Então:

1. se $f, g$ são monos, então $g \circ f$ é mono; 
2. se $g \circ f$ é mono, então $f$ é mono;

3. se $f, g$ são epis, então $g \circ f$ é epi;

4. se $g \circ f$ é epi, então $g$ é epi.

Definição 1.2.4. Sejam $\mathcal{C}$ uma categoria e $m, e \in \mathcal{C}_{1}$. A flecha $m: a \rightarrow b$ é dita uma $\boldsymbol{s e c ̧ a ̃ o ~}$ se existe $f: b \rightarrow a$ em $\mathcal{C}_{1}$ tal que $f \circ m=i d_{a}$ (note que, em particular, $m$ é mono). A flecha $e: a \rightarrow b$ é dita uma retração se existe $g: b \rightarrow a$ em $\mathcal{C}_{1}$ tal que $e \circ g=i d_{b}$ (note que, em particular, e é epi).

Definição 1.2.5. Sejam $\mathcal{C}$ uma categoria e $f: a \rightarrow b$ em $\mathcal{C}_{1}$. Dizemos que $f$ possui fatoração epi-mono se existirem um epi $f^{\triangleright}: a \rightarrow f(a)$ e um mono $i m(f): f(a) \longmapsto b$ tais que $f=$ $i m(f) \circ f^{\triangleright}$; e para todo epi $u: a \rightarrow c$ e mono $v: c \longmapsto b$ tais que $f=v \circ u$, existe um único iso $\kappa: f(a) \rightarrow c$ que torna o diagrama abaixo comutativo:

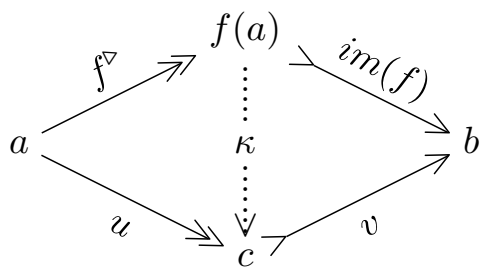

Como a notação sugere, é comum chamar a flecha $i m(f)$ de imagem de $f$.

Exemplo 1.2.6. Toda flecha em Set possui fatoração epi-mono: dada uma função $f: A \rightarrow B$, defina $f^{\triangleright}: A \rightarrow f(A)$ como a imagem direta de $f$ (isto é, $f(A)=\{f(a) \mid a \in A\}$ e $f^{\triangleright}(a)=f(a)$ ), e $i m(f): f(A) \rightarrow B$ como a inclusão $i_{f(A)}: f(A) \hookrightarrow B$.

Definição 1.2.7. Seja $\mathcal{C}$ uma categoria. Uma família $\mathcal{G} \subseteq \mathcal{C}_{0}$ é dita uma família (ou subclasse) geradora quando, dadas $f, g: a \rightarrow b$ em $\mathcal{C}_{1}$, se para todo $u \in \mathcal{G}$ e toda flecha $h: u \rightarrow a$ em $\mathcal{C}_{1}$ vale $f \circ h=g \circ h$, então $f=g$.

Exemplo 1.2.8. Em Set, todo conjunto unitário (aliás, todo conjunto não vazio) é gerador. Em Grp e $\mathbf{A b},\{(\mathbb{Z},+)\}$ é gerador. Dado um anel $R,\{R\}$ é conjunto gerador em $R$ - Mod, a categoria dos $R$-módulos e seus homomorfismos (tanto nesse exemplo como no anterior para grupos, a ideia é que homomorfismos com domínio no gerador serão determinados por como agem na unidade).

Definição 1.2.9. Sejam $\mathcal{C}$ uma categoria e $a, b \in \mathcal{C}_{0}$. O objeto $a$ é dito inicial se, para todo $c \in \mathcal{C}_{0}$, existe uma única flecha $f \in \mathcal{C}_{1}$ tal que $f: a \rightarrow c$. O objeto $b$ é dito terminal se, para todo $c \in \mathcal{C}_{0}$, existe uma única flecha $g \in \mathcal{C}_{1}$ tal que $g: c \rightarrow b$. 
É fácil ver que tanto objetos iniciais quanto objetos terminais são únicos a menos de isomorfismo. Por isso, será comum denotar, em uma dada categoria, o objeto inicial por $\mathbf{0}$ e o terminal por 1; e as flechas únicas da definição serão denotadas por $\mathrm{O}_{c}: \mathbf{0} \rightarrow c$ e $\mathrm{I}_{c}: c \rightarrow \mathbf{1}$.

Exemplo 1.2.10. Em Set, $\emptyset$ é o (único) objeto inicial, enquanto todo conjunto unitário é objeto terminal. Em Grp, o grupo trivial é tanto objeto inicial como terminal. Em Ring, $\mathbb{Z}$ é objeto inicial e o anel trivial é objeto terminal.

\subsection{Transformações naturais}

Tal como funtores podem ser compreendidos como morfismos de categorias, transformações naturais são definidas como um "morfismo entre funtores": uma forma de transformar um funtor em outro preservando a estrutura das categorias envolvidas.

Definição 1.3.1. Sejam $\mathcal{A}, \mathcal{B}$ categorias e $F, G: \mathcal{A} \rightarrow \mathcal{B}$ funtores. Uma transformação natural $\eta: F \Rightarrow G$ é uma família $\eta=\left(\eta_{a}\right)_{a \in \mathcal{A}_{0}}$, onde $\eta_{a}: F_{0}(a) \rightarrow G_{0}(a)$ em $\mathcal{B}_{1}$ para cada $a \in \mathcal{A}_{0}$, satisfazendo $\eta_{a^{\prime}} \circ F_{1}(f)=G_{1}(f) \circ \eta_{a}$ para todo $f: a \rightarrow a^{\prime}$ em $\mathcal{A}_{1}$, como ilustrado no diagrama comutativo:

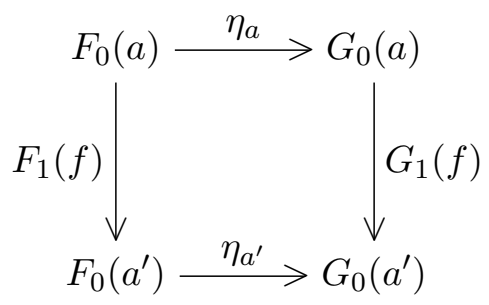

Se, para cada $a \in \mathcal{A}_{0}, \eta_{a}$ é um iso em $\mathcal{B}_{1}$, então $\eta$ é dita um isomorfismo natural.

\section{Exemplo 1.3.2.}

- Para cada conjunto $X$, podemos considerar o mapa unitário $\eta_{X}: X \rightarrow \mathcal{P}(X)$ tal que $\eta_{X}(x)=\{x\}$, para todo $x \in X$. Isso define uma transformação natural $\eta: i d_{\text {Set }} \Rightarrow \mathcal{P}$ entre o funtor identidade e o funtor partes;

- na categoria $\operatorname{Vect}_{K}$ dos $K$-espaços vetoriais (para $K$ um corpo) e transformações lineares, considere o funtor identidade $i d_{\mathbf{V e c t}_{K}}: \boldsymbol{V e c t}_{K} \rightarrow \operatorname{Vect}_{K}$ e o funtor bidual $(-)^{* *}$ : $\operatorname{Vect}_{K} \rightarrow \operatorname{Vect}_{K}$. Então, podemos definir uma transformação natural $\eta: i d_{\operatorname{Vect}_{K}} \Rightarrow(-)^{* *}$ fazendo $\eta_{V}=V \rightarrow V^{* *}, \eta_{V}(v)=e v_{v}$ (a avaliação em $v$ ), para todo $v \in V$;

- considere o funtor $(-)^{\times}: \mathbf{c R n g} \rightarrow \mathbf{G r p}$ o funtor que leva cada $R \in \mathbf{c R n g}_{0}$ ao grupo $R^{\times}$dos elementos invertíveis de $R$ (e os homomorfismos a sua restrição). Então, o determinante 


$$
\begin{aligned}
& \operatorname{det}_{R}: G L_{n}(R) \rightarrow R^{\times} \text {para cada } R \in \mathbf{c R n g}_{0} \text { define uma transformação natural det : } \\
& G L_{n} \Rightarrow(-)^{\times} .
\end{aligned}
$$

Definição 1.3.3. Sejam $\mathcal{C}$ uma categoria e $F: \mathcal{C} \rightarrow$ Set um funtor. Dizemos que $F$ é representável se existem $c \in \mathcal{C}_{0}$ e um isomorfismo natural $\eta: F \Rightarrow \mathcal{C}(c,-)$.

Definição 1.3.4. Sejam $\mathcal{A}, \mathcal{B}, \mathcal{C}, \mathcal{D}$ categorias, $F, G: \mathcal{A} \rightarrow \mathcal{B}, H: \mathcal{B} \rightarrow \mathcal{C}$ e $K: \mathcal{D} \rightarrow \mathcal{A}$ funtores, e $\eta: F \Rightarrow G$ uma transformação natural. Então, definimos a transformação natural $H \eta: H \circ F \Rightarrow H \circ G$ fazendo

$$
(H \eta)_{a}=H_{1}\left(\eta_{a}\right): H_{0}\left(F_{0}(a)\right) \rightarrow H_{0}\left(G_{0}(a)\right) \text {, para todo } a \in \mathcal{A}_{0}
$$

Similarmente, definimos a transformação natural $\eta K: F \circ K \Rightarrow G \circ K$ fazendo

$$
(\eta K)_{d}=\eta_{K_{0}(d)}: F_{0}\left(K_{0}(d)\right) \rightarrow G_{0}\left(K_{0}(d)\right), \text { para todo } d \in \mathcal{D}_{0}
$$

Definição 1.3.5. Sejam $\mathcal{A}, \mathcal{B}$ categorias, $F, G, H: \mathcal{A} \rightarrow \mathcal{B}$ funtores, e $\eta: F \Rightarrow G$ e $\theta: G \Rightarrow H$ transformações naturais. Definimos a composição $\theta \circ \eta: F \Rightarrow H$ fazendo, para cada $a \in \mathcal{A}$, $(\theta \circ \eta)_{a}:=\theta_{a} \circ \eta_{a}{ }^{8}$

Tendo definido a composição, é fácil definir a transformação natural identidade $i d_{F}: F \Rightarrow F$ : basta tomar $\left(i d_{F}\right)_{a}=i d_{F(a)}$ para todo $a \in \mathcal{A}$.

Definição 1.3.6. Sejam $\mathcal{A}, \mathcal{B}$ categorias. A categoria de funtores de $\mathcal{A}$ para $\mathcal{B}$, denotada $\mathcal{B}^{\mathcal{A}}($ ou $[\mathcal{A}, \mathcal{B}])$, é a categoria que tem como objetos os funtores $F: \mathcal{A} \rightarrow \mathcal{B}$ e como flechas as transformações naturais entre eles.

Observe que, dado um funtor $F: \mathcal{A} \rightarrow \mathcal{C}^{\mathcal{B}}$, para cada $b \in \mathcal{B}_{0}$ podemos definir um funtor $F(-)(b): \mathcal{A} \rightarrow \mathcal{C}$ fazendo:

$$
\begin{gathered}
(F(-)(b))_{0}(a):=\left(F_{0}(a)\right)_{0}(b), \text { para todo } a \in \mathcal{A}_{0} \\
(F(-)(b))_{0}(f):=\left(F_{1}(f)\right)_{b}:\left(F_{0}(a)\right)_{0}(b) \rightarrow\left(F_{0}\left(a^{\prime}\right)\right)_{0}(b), \text { para toda } f: a \rightarrow a^{\prime} \text { em } \mathcal{A}_{1}
\end{gathered}
$$

Como a notação com todos os subscritos (para diferenciar entre o funtor agindo nos objetos e o funtor agindo nas flechas) fica muito carregada, nesse caso será comum suprimi-los e denotar, por exemplo, $\left(F_{0}(a)\right)_{0}(b)$ simplesmente por $F(a)(b)$.

\footnotetext{
${ }^{8}$ Essa composição é às vezes chamada de composição vertical, para distinguir da composição horizontal entre transformações naturais $\eta: F \Rightarrow G$ e $\theta: H \Rightarrow K$, para funtores $F, G: \mathcal{A} \rightarrow \mathcal{B}$ e $H, K: \mathcal{B} \rightarrow \mathcal{C}$.
} 
Definição 1.3.7. Sejam $\mathcal{A}, \mathcal{B}$ categorias e $F: \mathcal{A} \rightarrow \mathcal{B}$ um funtor. $F$ é dito uma equivalência de categorias se existem $G: \mathcal{B} \rightarrow \mathcal{A}$ um funtor e isomorfismos naturais $\eta: i d_{\mathcal{A}} \Rightarrow G \circ F$ e $\theta: i d_{\mathcal{B}} \Rightarrow F \circ G$ (ou, equivalentemente, se $F$ for pleno, fiel e, para cada $b \in \mathcal{B}$, existe $a \in \mathcal{A}$ e um iso $g: b \rightarrow F(a))$. Nesse caso, denotamos $\mathcal{A} \simeq \mathcal{B}$.

Essencialmente, a diferença entre isomorfismo e equivalência de categorias é que, no segundo, podemos ter quantidades diferentes de objetos isomorfos entre si. Por exemplo, considere as categorias $\mathcal{A}$ e $\mathcal{B}$ tais que:

$$
\begin{array}{cc}
\mathcal{A}_{0}=\{a\} & \mathcal{A}_{1}=\left\{i d_{a}\right\} \\
\mathcal{B}_{0}=\{a, b\} & \mathcal{B}_{1}=\left\{i d_{a}, i d_{b}, f: a \rightarrow b, f^{-1}: b \rightarrow a\right\}
\end{array}
$$

com $a \neq b$ (e $f$, tendo inversa, é um iso). Apesar da semelhança, essas categorias não são isomorfas (para qualquer funtor $F: \mathcal{A} \rightarrow \mathcal{B}, F_{0}$ não tem como ser uma bijeção). Porém, é fácil definir uma equivalência de categorias: seja $F: \mathcal{A} \rightarrow \mathcal{B}$ o funtor tal que $F_{0}(a)=a$ e $F_{1}\left(i d_{a}\right)=i d_{a} . F$ é claramente pleno e fiel, e existem isos $i d_{a}: a \rightarrow F(a)$ e $f^{-1}: b \rightarrow F(a)$.

Para um exemplo mais concreto disso, $\mathbf{S e t}_{\text {fin }}$ e $\mathbf{O r d}_{f i n}$, a categoria dos conjuntos finitos e a categoria dos ordinais finitos (respectivamente), são categorias equivalentes, mas não isomorfas: ao contrário de $\mathbf{S e t}_{f i n}$, Ord $_{\text {fin }}$ é uma categoria pequena. ${ }^{9}$

Teorema 1.3.8. Lema de Yoneda. Sejam $\mathcal{C}$ uma categoria localmente pequena, $F: \mathcal{C} \rightarrow$ Set um funtor e $c \in \mathcal{C}_{0}$. Então, existe uma bijeção $y_{(F, c)}: \operatorname{Nat}(\mathcal{C}(c,-), F) \rightarrow F_{0}(c)$ entre as transformações naturais de $\mathcal{C}(c,-)$ em $F$ e o conjunto $F_{0}(c)$.

Explicitamente, a bijeção é dada por

$$
y_{(F, c)}(\eta)=\eta_{c}\left(i d_{c}\right), \text { para toda transformação natural } \eta: \mathcal{C}(c,-) \Rightarrow F
$$

Já sua inversa $z: F_{0}(c) \rightarrow \operatorname{Nat}(\mathcal{C}(c,-), F)$ é definida fazendo-se, para cada $a \in F_{0}(c), z(a)$ : $\mathcal{C}(c,-) \Rightarrow F$ é a transformação natural com componentes

$$
z(a)_{b}: \mathcal{C}(c, b) \rightarrow F_{0}(b), \text { para cada } b \in \mathcal{C}_{0}, \text { com }
$$

$$
z(a)_{b}(f)=F_{1}(f)(a), \text { para todo } f: c \rightarrow b \text { em } \mathcal{C}_{1}
$$

\footnotetext{
${ }^{9}$ Aliás, Ord $_{\text {fin }}$ é o "esqueleto" de Set $_{\text {fin }}$ : uma subcategoria esquelética (isto é, todo iso é igualdade) tal que o funtor inclusão é uma equivalência de categorias.
} 
Aliás, vale ainda mais: a bijeção do Lema de Yoneda é natural em $F$ e em $c$, de forma que obtemos uma versão mais geral.

Teorema 1.3.9. Seja $\mathcal{C}$ uma categoria localmente pequena. Considere os bifuntores $E, N$ : Set $^{\mathcal{C}} \times \mathcal{C} \rightarrow$ Set dados por:

$$
\begin{gathered}
E_{0}(F, c):=F_{0}(c) \\
E_{1}(\eta, f): F(c) \rightarrow G\left(c^{\prime}\right), E_{1}(\eta, f):=G_{1}(f) \circ \eta_{c} \\
N_{0}(F, c):=\operatorname{Nat}(\mathcal{C}(c,-), F) \\
N_{1}(\eta, f): N a t(\mathcal{C}(c,-), F) \rightarrow \operatorname{Nat}\left(\mathcal{C}\left(c^{\prime},-\right), G\right), N_{1}(\eta, f)(\theta):=\eta \circ \theta \circ \mathcal{C}(f,-)
\end{gathered}
$$

para todos $c, c^{\prime} \in \mathcal{C}_{0}, f: c \rightarrow c^{\prime}$ em $\mathcal{C}_{1}, F, G \in\left(\mathbf{S e t}^{\mathcal{C}}\right)_{0}, \eta: F \Rightarrow G$ em $\left(\mathbf{S e t}^{\mathcal{C}}\right)_{1}$ e $\theta \in$ $\operatorname{Nat}(\mathcal{C}(c,-), F)$. Então, $y: N \Rightarrow E$ é um isomorfismo natural (onde cada componente é a bijeção $y_{(F, c)}$ do Lema de Yoneda).

Para a demonstração detalhada, pode-se consultar o teorema 1.3.3 de [Bor08a] ou a seção 1.5 de [BW85].

Definição 1.3.10. Seja $\mathcal{C}$ uma categoria pequena. O Funtor de Yoneda $\mathcal{Y}_{\mathcal{C}^{o p}}: \mathcal{C}^{o p} \rightarrow$ Set $^{\mathcal{C}}$ é o funtor tal que:

$$
\left(\mathcal{Y}_{\mathcal{C}^{o p}}\right)_{0}(c):=\mathcal{C}(c,-), \text { para todo } c \in \mathcal{C}_{0}
$$

$$
\begin{aligned}
\left(\mathcal{Y}_{\mathcal{C}^{o p}}\right)_{1}(f):=\mathcal{C}(f,-): \mathcal{C}(c,-) \Rightarrow \mathcal{C}\left(c^{\prime},-\right), \text { para toda } f: c \rightarrow c^{\prime} \text { em } \mathcal{C}_{1}, \\
\text { onde } \mathcal{C}(f,-)_{a}:=\mathcal{C}(f, a), \text { para cada } a \in \mathcal{C}_{0}
\end{aligned}
$$

Às vezes esse funtor é chamado de Funtor de Yoneda contravariante, para distinguir de quando a categoria é trocada por sua oposta e obtemos o "Funtor de Yoneda covariante" $\mathcal{Y}_{\mathcal{C}}: \mathcal{C} \rightarrow$ Set $^{\mathcal{C}^{o p}}$. O Lema de Yoneda garante que esses funtores sejam plenos e fieis.

De fato, para todos $a, b \in \mathcal{C}_{0}$, temos:

$$
\operatorname{Set}^{\mathcal{C}}\left(\left(\mathcal{Y}_{\mathcal{C}^{o p}}\right)_{0}(a),\left(\mathcal{Y}_{\mathcal{C}^{o p}}\right)_{0}(b)\right)=\operatorname{Nat}(\mathcal{C}(a,-), \mathcal{C}(b,-)) \cong \mathcal{C}(b, a)=\mathcal{C}^{o p}(a, b)
$$

Como corolário imediato do Funtor de Yoneda ser pleno e fiel, temos:

Corolário 1.3.11. Sejam $\mathcal{C}$ uma categoria pequena e $a, a^{\prime} \in \mathcal{C}_{0}$. Se $\mathcal{C}(a, c) \cong \mathcal{C}\left(a^{\prime}, c\right)$ para todo $c \in \mathcal{C}_{0}$, então $a \cong a^{\prime}$. 
Naturalmente, esse resultado pode ser dualizado (basta substituir $\mathcal{C}$ por $\mathcal{C}^{o p}$ ).

\subsection{Limites}

Limites aparecem com frequência em diversos contextos matemáticos, porém sua definição precisa é bastante abstrata. Intuitivamente, um limite de um diagrama pode ser pensado como um "conjunto solução" de várias equações, onde cada coordenada é representada por um objeto do diagrama, e as equações são representadas pelos morfismos do diagrama.

Usamos cones para formalizar essa ideia de "conjunto solução", e definimos um limite como um cone "universal", isto é, como a "solução ótima". Os exemplos apresentados nessa seção ilustram o que a noção de limite busca generalizar.

Definição 1.4.1. Sejam $\mathcal{A}, \mathcal{B}$ categorias e $F: \mathcal{A} \rightarrow \mathcal{B}$ um funtor. Um cone sobre $F$ é um par $\left(b,\left(\varphi_{a}\right)_{a \in \mathcal{A}_{0}}\right)$, onde $b \in \mathcal{B}_{0}$ e $\varphi_{a}: b \rightarrow F_{0}(a)$ em $\mathcal{B}_{1}$, para cada $a \in \mathcal{A}_{0}$, tal que $\varphi_{a^{\prime}}=F_{1}(f) \circ \varphi_{a}$, para todo $f: a \rightarrow a^{\prime}$ em $\mathcal{A}_{1}$, como no diagrama comutativo:

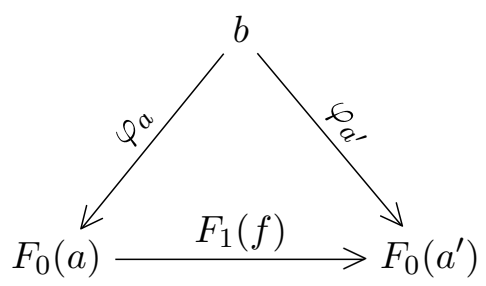

Um cocone sobre $F$ é a noção dual:

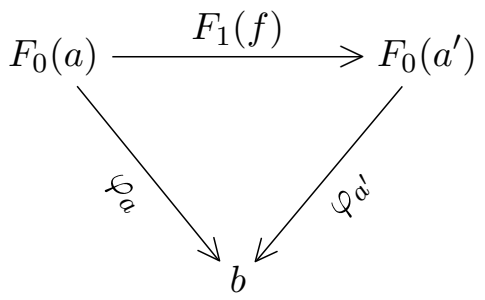

No restante dessa seção, será comum, como fizemos acima, apenas indicar o diagrama comutativo que ilustra a definição da noção dual, pois escrever as definições precisas apenas tornaria o texto desnecessariamente cansativo.

Definição 1.4.2. Sejam $\mathcal{A}, \mathcal{B}$ categorias e $F: \mathcal{A} \rightarrow \mathcal{B}$ um funtor. Um limite de $F$ (se existir) é um cone $\left(l,\left(\psi_{a}\right)_{a \in \mathcal{A}_{0}}\right)$ sobre $F$ tal que, para todo cone $\left(b,\left(\varphi_{a}\right)_{a \in \mathcal{A}_{0}}\right)$ sobre $F$, existe um único 
$\kappa: b \rightarrow l$ em $\mathcal{B}_{1}$ tal que $\varphi_{a}=\psi_{a} \circ \kappa$, para todo $a \in \mathcal{A}_{0}$, como no diagrama comutativo:

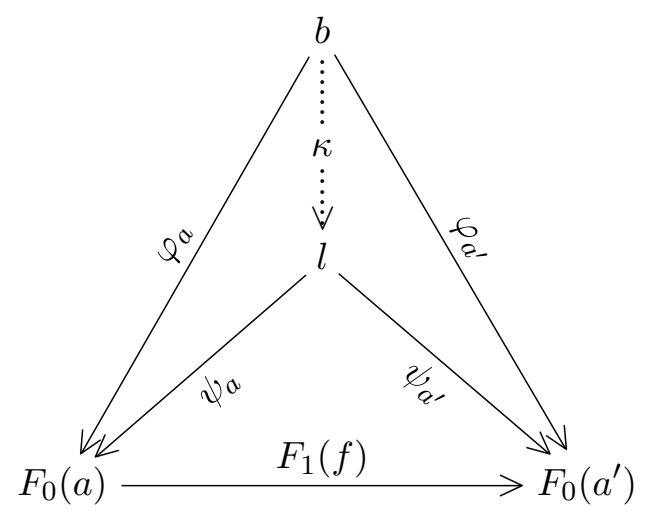

Similarmente, um colimite de $F$ (se existir) é a noção dual:

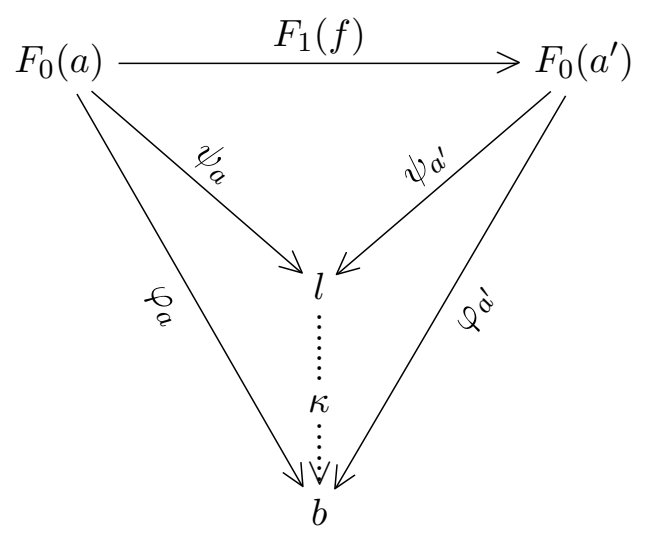

Podemos definir um morfismo de cones $\Phi:\left(b,\left(\varphi_{a}\right)_{a \in \mathcal{A}_{0}}\right) \rightarrow\left(b^{\prime},\left(\varphi_{a}^{\prime}\right)_{a \in \mathcal{A}_{0}}\right)$ como uma flecha $\Phi: b \rightarrow b^{\prime}$ em $\mathcal{B}_{1}$ tal que, para todo $a \in \mathcal{A}_{0}, \varphi_{a}=\varphi_{a}^{\prime} \circ \Phi$, como no diagrama comutativo:

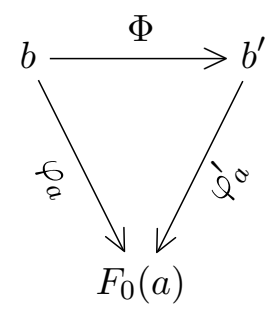

Dualmente, os morfismos de cocones são representados pelo diagrama comutativo:

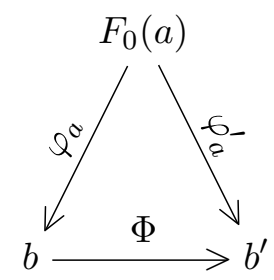

Dessa forma, podemos definir a categoria dos $\operatorname{Cone}(F)$ cones sobre $F$ e seus morfismos, e a categoria Cocone $(F)$ dos cocones sobre $F$ e seus morfismos. 
Observe, portanto, que os limites são objetos iniciais em $\operatorname{Cone}(F)$, e colimites são objetos iniciais em Cocone $(F)$. Isso garante que limites e colimites (quando existirem) sejam únicos a menos de isomorfismo.

Antes de começarmos os exemplos, registremos uma definição, que aparecerá mais à frente, de um tipo especial de família de flechas:

Definição 1.4.3. Seja $\mathcal{C}$ uma categoria. Uma família $\left\{h_{i}: u_{i} \rightarrow a \mid i \in I\right\} \subseteq \mathcal{C}_{1}$ é dita epimórfica quando, dadas $f, g: a \rightarrow b$ em $\mathcal{C}_{1}$, se $f \circ h_{i}=g \circ h_{i}$ para todo $i \in I$, então $f=g$.

Para um exemplo, note que a unicidade da definição de um colimite $\left(l,\left(\psi_{a}\right)_{a \in \mathcal{A}_{0}}\right)$ implica em as flechas $\left(\psi_{a}\right)_{a \in \mathcal{A}_{0}}$ formarem uma família epimórfica.

Além disso, é fácil ver que, se $\mathcal{G} \subseteq \mathcal{C}_{0}$ é uma família geradora, então para todo $a \in \mathcal{C}_{0}$ as flechas $\{f: u \rightarrow a \mid u \in \mathcal{G}\} \subseteq \mathcal{C}_{1}$ constituem uma família epimórfica.

Exemplo 1.4.4. Seja $I$ um conjunto. Definamos a categoria $\mathcal{I}$ tomando $\mathcal{I}_{0}=I$ (os objetos são os elementos do conjunto) e $\mathcal{I}_{1}=\left\{i d_{i} \mid i \in I\right\}$ (as flechas são apenas as identidades). Agora, sejam $\mathcal{C}$ uma categoria e $F: \mathcal{I} \rightarrow \mathcal{C}$ um funtor. Observe que podemos pensar no funtor $F$ como apenas representando uma família de objetos $\left(F_{0}(i)\right)_{i \in I}$ indexada por $I$ (observe, também, que como as flechas em $\mathcal{I}$ são apenas as identidades, a condição de cone é trivial). Nesse caso, o limite de $F$ é chamado de produto de $\left(F_{0}(i)\right)_{i \in I}$, e será denotado por $\left(\prod_{i \in I} F_{0}(i),\left(\pi_{i}\right)_{i \in I}\right) ; \pi_{i}$ serão chamadas de projeções.

Façamos explicitamente o produto binário: sejam $a, b \in \mathcal{C}_{0}$. Seu produto será um cone $\left(a \times b, \pi_{1}, \pi_{2}\right)$ com $\pi_{1}: a \times b \rightarrow a$ e $\pi_{2}: a \times b \rightarrow b$ em $\mathcal{C}_{1}$ tal que, para todo outro cone $\left(c, \gamma_{1}, \gamma_{2}\right)$ com $\gamma_{1}: c \rightarrow a$ e $\gamma_{2}: c \rightarrow b$ em $\mathcal{C}_{1}$, existe um único $\left\langle\gamma_{1}, \gamma_{2}\right\rangle: c \rightarrow a \times b$ em $\mathcal{C}_{1}$ tal que $\gamma_{1}=\pi_{1} \circ \kappa$ e $\gamma_{2}=\pi_{2} \circ \kappa$, como no diagrama comutativo:

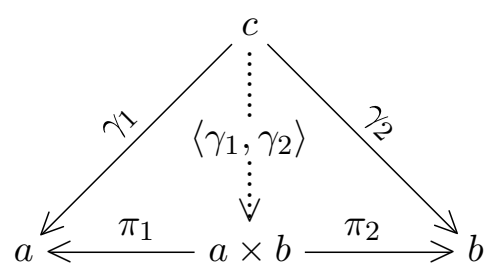

Dadas duas flechas $f: a \rightarrow c$ e $g: b \rightarrow d$, definimos a flecha produto como $f \times g:=\left\langle f \circ \pi_{1}, g \circ \pi_{2}\right\rangle$ : $a \times b \rightarrow c \times d$ (onde $\pi_{1}, \pi_{2}$ são as projeções do produto $a \times b$ ). Além disso, dados os produtos finitos $\left(\prod_{i=1}^{n} a_{i},\left(\pi_{i}\right)_{1 \leq i \leq n}\right)$ e $\left(\prod_{i=1}^{n+m} a_{i},\left(\rho_{i}\right)_{1 \leq i \leq n+m}\right)(\operatorname{com} n, m \in \mathbb{N})$, definimos a projeção nas $n$ primeiras coordenadas $\pi: \prod_{i=1}^{n+m} a_{i} \rightarrow \prod_{i=1}^{n} a_{i}$ como a flecha única tal que $\pi_{i} \circ \pi=\rho_{i}$ para todo $i \in\{1, \ldots, n\}$ (projeções em outras escolhas de coordenadas são definidas de forma análoga). 
Exemplo 1.4.5. Alguns exemplos de produtos:

- em Set, o produto corresponde ao produto cartesiano de conjuntos, com as projeções:

$$
\pi_{j}: \prod_{i \in I} X_{i} \rightarrow X_{j} \quad \pi_{j}\left(\left(x_{i}\right)_{i \in I}\right)=x_{j}
$$

- se $(P, \leq)$ é um conjunto preordenado considerado como uma categoria, então o produto corresponde ao ínfimo (as projeções correspondem a $\bigwedge_{i \in I} x_{i} \leq x_{j}$ );

- em Top, o produto corresponde ao produto cartesiano munido da topologia produto, que é a menor topologia na qual as projeções (definidas como em Set) são contínuas;

- em Grp, o produto corresponde ao produto direto de grupos (ou seja, produto cartesiano com operações definidas componente a componente), e novamente as projeções são definidas como em Set.

Exemplo 1.4.6. A noção dual ao produto é chamada de coproduto, que será denotado por $\left(\coprod_{i \in I} F_{0}(i),\left(\iota_{i}\right)_{i \in I}\right)$; dizemos que $\iota_{i}$ são as injeções canônicas. O coproduto binário de dois objetos $a, b \in \mathcal{C}_{0}$ será denotado por $\left(a+b, \iota_{1}, \iota_{2}\right)$, e podemos representá-lo pelo diagrama:

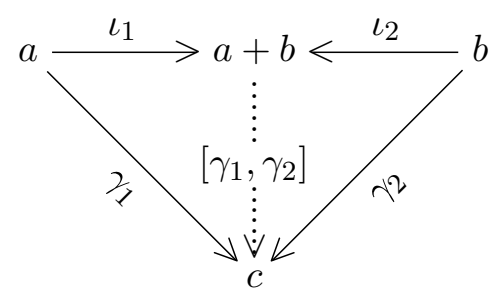

Mais uma vez, dadas duas flechas $f: a \rightarrow c$ e $g: b \rightarrow d$, definimos a flecha coproduto como $f+g:=\left[\iota_{1} \circ f, \iota_{2} \circ g\right]: a+b \rightarrow c+d$ (onde $\iota_{1}, \iota_{2}$ são as injeções canônicas do coproduto $c+d$ ).

Exemplo 1.4.7. Alguns exemplos de coprodutos:

- em Set, o coproduto corresponde à união disjunta conjuntos, com as injeções canônicas:

$$
\iota_{j}: X_{j} \rightarrow \bigsqcup_{i \in I} X_{i} \quad \iota_{j}(x)=(x, j)
$$

- se $(P, \leq)$ é um conjunto preordenado considerado como uma categoria, então o coproduto corresponde ao supremo (as injeções canônicas correspondem a $x_{j} \leq \bigvee_{i \in I} x_{i}$ );

- em Ab, o coproduto corresponde à soma direta de grupos abelianos, com as injeções 
canônicas:

$$
\iota_{j}: A_{j} \rightarrow \bigoplus_{i \in I} A_{i} \quad \iota_{j}(a)=\left(a_{i}\right)_{i \in I}
$$

com $a_{j}=a$ e $a_{i}=0$, para $i \neq j$.

Exemplo 1.4.8. Considere a categoria $\mathcal{A}$ com três objetos $\mathcal{A}_{0}=\left\{a_{1}, a_{2}, a_{3}\right\}$ e com duas flechas diferentes das identidades $\mathcal{A}_{1}=\left\{i d_{a_{1}}, i d_{a_{2}}, i d_{a_{3}}, \alpha_{1}: a_{1} \rightarrow a_{3}, \alpha_{2}: a_{2} \rightarrow a_{3}\right\}$. Dada uma categoria $\mathcal{B}$, um funtor $F: \mathcal{A} \rightarrow \mathcal{B}$ basicamente fornece os objetos $F_{0}\left(a_{1}\right)=a, F_{0}\left(a_{2}\right)=b, F_{0}\left(a_{3}\right)=c$ em $\mathcal{B}_{0}$ e duas flechas $f=F_{1}\left(\alpha_{1}\right)$ e $g=F_{1}\left(\alpha_{2}\right)$ em $\mathcal{B}_{1}$. O limite de $F$ é chamado de pullback de $f$ e $g$, e será denotado por $\left(p, f^{\prime}, g^{\prime}\right)$, onde $f^{\prime}: p \rightarrow b$ e $g^{\prime}: p \rightarrow a$ em $\mathcal{B}_{1}$ são tais que $f \circ g^{\prime}=g \circ f^{\prime}$ (para garantir a condição de cone). A rigor, essa tripla sozinha não é um cone, mas está determinada por ela: a flecha $\psi_{c}$ que falta será $f \circ g^{\prime}$.

Assim, para qualquer outra tripla $\left(d, \delta_{1}, \delta_{2}\right)$ com $\delta_{1}: d \rightarrow a$ e $\delta_{2}: d \rightarrow b$ em $\mathcal{B}_{1}$ tais que $f \circ \delta_{1}=g \circ \delta_{2}$, existe uma única $\kappa: d \rightarrow p$ em $\mathcal{B}_{1}$ tal que $\delta_{1}=g^{\prime} \circ \kappa$ e $\delta_{2}=f^{\prime} \circ \kappa$, como no diagrama comutativo:

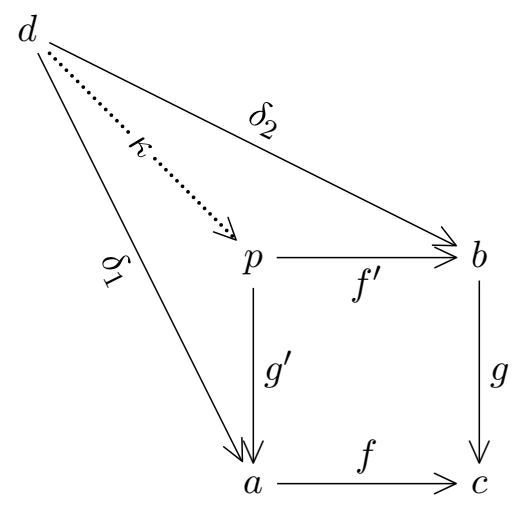

Algumas denominações usuais: dizemos que o diagrama formado pelas flechas $f, g, f^{\prime}, g^{\prime}$, com vértices $p, a, b, c$, forma um quadrado (ou retângulo, se a figura do diagrama assim o sugerir) de pullback; e dizemos que $f^{\prime}$ é o pullback de $f$ ao longo de $g$.

Exemplo 1.4.9. Em Set, o pullback de duas funções $f: a \rightarrow c, g: b \rightarrow c$ será:

$$
\begin{aligned}
& p=\{(x, y) \in a \times b \mid f(x)=g(y)\} \\
& f^{\prime}(x, y)=y \quad g^{\prime}(x, y)=x
\end{aligned}
$$

Exemplo 1.4.10. Similarmente, podemos obter o pushout, a noção dual ao pullback. Deno- 
tando o pushout pela tripla $\left(q, i_{1}, i_{2}\right)$, podemos representá-lo pelo diagrama:

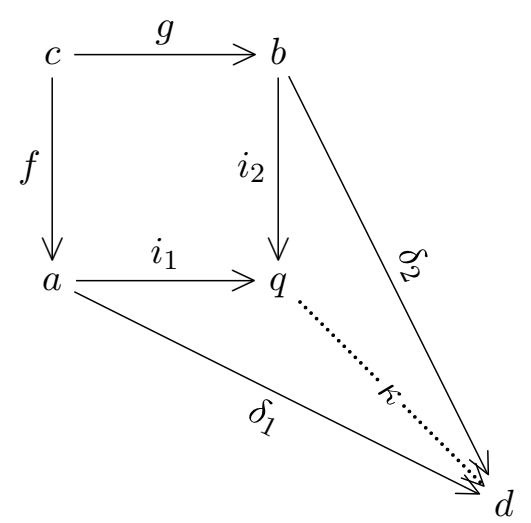

Exemplo 1.4.11. Em Set, dadas duas funções $f: c \rightarrow a, g: c \rightarrow b$, considere a relação de equivalência $\equiv$ dada por, para todos $x \in a$ e $y \in b$,

$$
x \equiv y \quad \text { se, e somente se, } \quad \text { existe } z \in c \text { tal que } f(z)=x \text { e } g(z)=y
$$

Então, o pushout de $f$ e $g$ será:

$$
\begin{gathered}
q=\frac{a \sqcup b}{\equiv} \\
i_{1}(x)=[x] \quad i_{2}(y)=[y]
\end{gathered}
$$

Exemplo 1.4.12. Considere a categoria $\mathcal{A}$ com dois objetos $\mathcal{A}_{0}=\left\{a_{1}, a_{2}\right\}$ e duas flechas diferentes das identidades $\mathcal{A}_{1}=\left\{i d_{a_{1}}, i d_{a_{2}}, \alpha_{1}, \alpha_{2}: a_{1} \rightarrow a_{2}\right\}$. Dada uma categoria $\mathcal{B}$, um funtor $F: \mathcal{A} \rightarrow \mathcal{B}$ basicamente fornece os objetos $F_{0}\left(a_{1}\right)=a, F_{0}\left(a_{2}\right)=b$ em $\mathcal{B}_{0}$ e duas flechas $F_{1}\left(\alpha_{1}\right)=f, F_{1}\left(\alpha_{2}\right)=g$ em $\mathcal{B}_{1}$. O limite de $F$ é chamado de equalizador de $f$ e $g$, e será denotado por $(e, \varepsilon)$, onde $\varepsilon: e \rightarrow a$ satisfaz $f \circ \varepsilon=g \circ \varepsilon$ (para garantir condição de cone). Novamente, esse par não é, a rigor, um cone, a flecha $\psi_{b}$ que falta será $f \circ \varepsilon$.

Assim, para qualquer outro par $(d, \delta), \operatorname{com} \delta: d \rightarrow a$ tal que $f \circ \delta=g \circ \delta$ em $\mathcal{B}_{1}$, existe uma única $\kappa: d \rightarrow e$ em $\mathcal{B}_{1}$ tal que $\delta=\varepsilon \circ \kappa$, como no diagrama comutativo:

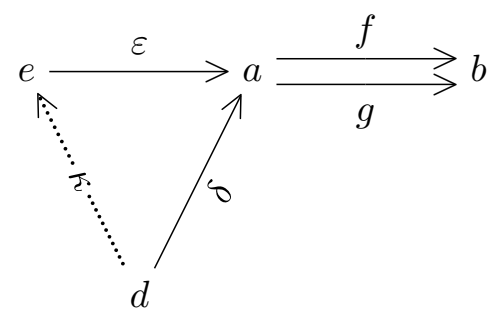

Exemplo 1.4.13. Novamente, com uma construção semelhante, poderíamos obter a noção dual da definição anterior: o coequalizador. Denotando o coequalizador por $(q, \varrho)$, podemos 
representá-lo pelo diagrama comuitativo:

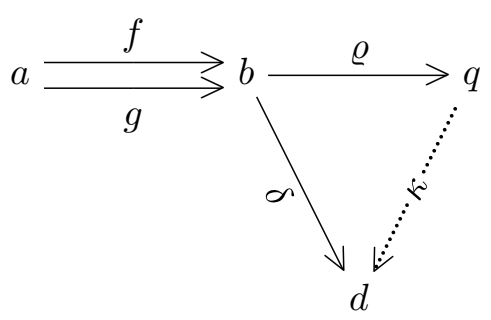

Exemplo 1.4.14. Objetos terminais e iniciais também podem ser vistos como limites e colimites. De fato, seja $\mathcal{C}$ uma categoria, $\mathbb{O}$ a categoria vazia e $F: \mathbb{O} \rightarrow \mathcal{C}$ um funtor. Dessa forma, cones e cocones sobre $F$ são simplesmente objetos de $\mathcal{C}$. Assim, um limite de $F$ é um objeto $b \in \mathcal{C}_{1}$ tal que, para todo objeto $c \in \mathcal{C}_{0}$, existe uma única flecha $f: c \rightarrow b$; ou seja, exatamente a definição de $b$ ser um objeto terminal. Similarmente, um colimite será um objeto inicial.

Definição 1.4.15. Seja $\mathcal{C}$ uma categoria. Dizemos que $\mathcal{C}$ é completa se todo funtor $F: \mathcal{A} \rightarrow \mathcal{C}$, $\operatorname{com} \mathcal{A}$ uma categoria pequena, possui limite. Dizemos que $\mathcal{C}$ é finitamente completa se todo funtor $F: \mathcal{B} \rightarrow \mathcal{C}, \operatorname{com} \mathcal{B}$ uma categoria finita, possui limite.

De forma similar poderíamos definir as noções duais de categorias cocompletas e finitamente cocompletas.

Definição 1.4.16. Sejam $\mathcal{A}, \mathcal{B}$ categorias e $F: \mathcal{A} \rightarrow \mathcal{B}$ um funtor. Dizemos que $F$ preserva limites quando, para toda categoria pequena $\mathcal{C}$ e todo funtor $G: \mathcal{C} \rightarrow \mathcal{A}$, se $G$ possui um limite $\left(l,\left(\psi_{c}\right)_{c \in \mathcal{C}_{0}}\right)$, então $\left(F_{0}(l),\left(F_{1}\left(\psi_{c}\right)\right)_{c \in \mathcal{C}_{0}}\right)$ é um limite de $F \circ G$.

Uma notação frequente e sugestiva é denotar o limite de um funtor $G: \mathcal{C} \rightarrow \mathcal{A}$ por $\lim _{c \in \mathcal{C}_{0}} G(c)$, de forma $F: \mathcal{A} \rightarrow \mathcal{B}$ preservar limites pode ser denotado por:

$$
F\left(\lim _{c \in \mathcal{C}_{0}} G(c)\right)=\lim _{c \in \mathcal{C}_{0}} F(G(c))
$$

onde, como esperado, $\lim _{c \in \mathcal{C}_{0}} F(G(c))$ é o limite de $F \circ G$.

\subsection{Alguns resultados sobre limites}

Listamos aqui resultados diversos sobre limites, mas, como mencionado no início da seção, omitimos a maioria das demonstrações. Aos leitores interessados nas provas, recomendamos o capítulo 2 de [Bor08a] por reunir resultados sobre limites de forma sistemática (embora também possam ser encontrados nos outros livros citados acima como referência bibliográfica). 
Proposição 1.5.1. Lema do pullback. Considere o diagrama:

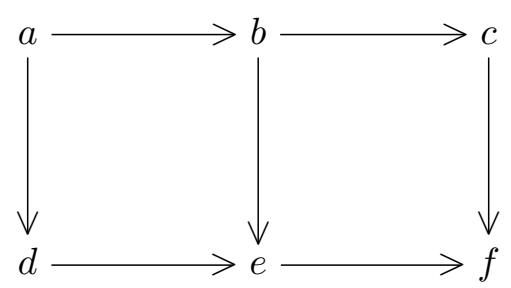

para quaisquer objetos $a, b, c, d, e, f$ de uma categoria $\mathcal{C}$. Então,

1. se $(a, b, e, d)$ e $(b, c, f, e)$ forem quadrados de pullback, então $(a, c, f, d)$ é um retângulo de pullback;

2. se $(b, c, f, e)$ for um quadrado de pullback e $(a, c, f, d)$ for um retângulo de pullback, então $(a, b, e, d)$ é um quadrado de pullback.

Proposição 1.5.2. Sejam $\mathcal{C}$ uma categoria, e $f: a \rightarrow c$ mono e $g: b \rightarrow c$ qualquer em $\mathcal{C}_{1}$. Então, o pullback $f^{\prime}$ de $f$ ao longo de g também é mono.

Proposição 1.5.3. Sejam $\mathcal{C}$ uma categoria e $f, g: a \rightarrow b$ em $\mathcal{C}_{1}$. Se $f$ e g possuem um equalizador $(e, \varepsilon)$, então $\varepsilon$ é um mono. Dualmente, se $f$ e g possuem um coequalizador $(q, \varrho)$, então $\varrho$ é um epi.

Com essa proposição, podemos definir tipos especiais de monos e epis:

Definição 1.5.4. Sejam $\mathcal{C}$ uma categoria e $m, e \in \mathcal{C}_{1}$. Dizemos que $m$ é um mono regular se for (a flecha de) um equalizador, e que e é um epi regular se for (a flecha de) um coequalizador.

Proposição 1.5.5. Sejam $\mathcal{C}$ uma categoria e $f \in \mathcal{C}_{1}$. Então,

1. se f for um mono regular e um epi, então $f$ é um iso;

2. se f for um epi regular e um mono, então $f$ é um iso.

Outra noção que será útil mais à frente é o kernel e o cokernel par:

Definição 1.5.6. Sejam $\mathcal{C}$ uma categoria e $f: a \rightarrow b$ em $\mathcal{C}_{1}$. O kernel par de $f$ é o pullback $(p, \alpha, \beta)$ de $f$ pela própria $f$, quando existir. Analogamente, o cokernel par de $f$ é o pushout $\left(q, i_{1}, i_{2}\right)$ de $f$ pela própria $f$.

Façamos alguns resultados sobre o kernel par, que naturalmente podem ser dualizados para o cokernel par. 
Proposição 1.5.7. Sejam $\mathcal{C}$ uma categoria e $f: a \rightarrow b$ em $\mathcal{C}_{1}$. Se o kernel par $(p, \alpha, \beta)$ de $f$ existir, então $\alpha$ e $\beta$ são epis.

Proposição 1.5.8. Sejam $\mathcal{C}$ uma categoria e $f: a \rightarrow b$ em $\mathcal{C}_{1}$. Então, são equivalentes:

1. $f$ é mono;

2. o kernel par de $f$ existe e é $\left(p, i d_{a}, i d_{a}\right)$;

3. o kernel par $(p, \alpha, \beta)$ de $f$ existe e $\alpha=\beta$.

Proposição 1.5.9. Sejam $\mathcal{C}$ uma categoria e $f: a \rightarrow b$ em $\mathcal{C}_{1}$ com kernel par $(p, \alpha, \beta)$. Se existe $(q, \varrho)$ um coequalizador de $\alpha$ e $\beta$, então $(p, \alpha, \beta)$ é o kernel par de $\varrho$.

Proposição 1.5.10. Sejam $\mathcal{C}$ uma categoria e $(q, \varrho)$ um coequalizador de duas flechas. Se $\varrho$ possui um kernel par $(p, \alpha, \beta)$, então $(q, \varrho)$ é um coequalizador de $\alpha$ e $\beta$.

Agora, resultados importantes sobre categorias completas e cocompletas:

Proposição 1.5.11. Seja $\mathcal{C}$ uma categoria. Então, $\mathcal{C}$ é completa se, e somente se, possui produtos arbitrários e equalizadores.

Proposição 1.5.12. Seja $\mathcal{C}$ uma categoria. Então, $\mathcal{C}$ é cocompleta se, e somente se, possui coprodutos arbitrários e coequalizadores.

Proposição 1.5.13. Seja $\mathcal{C}$ uma categoria. Então, são equivalentes:

1. $\mathcal{C}$ é finitamente completa;

2. $\mathcal{C}$ possui objeto terminal e pullbacks;

3. $\mathcal{C}$ possui objeto terminal, produtos binários e equalizadores.

Proposição 1.5.14. Seja $\mathcal{C}$ uma categoria. Então, são equivalentes:

1. $\mathcal{C}$ é finitamente cocompleta;

2. $\mathcal{C}$ possui objeto inicial e pushouts;

3. $\mathcal{C}$ possui objeto inicial, coprodutos binários e coequalizadores.

Finalmente, alguns resultados sobre funtores que preservam limites: 
Proposição 1.5.15. Sejam $\mathcal{C}$ uma categoria localmente pequena e $c \in \mathcal{C}_{0}$. Então, o funtor $\mathcal{C}(c,-): \mathcal{C} \rightarrow$ Set preserva limites.

Como esse resultado será bastante importante mais à frente, façamos um esboço de sua demonstração:

Demonstração. Sejam $\mathcal{B}$ uma categoria pequena, $F: \mathcal{B} \rightarrow \mathcal{C}$ um funtor com limite $\left(l,\left(\psi_{b}\right)_{b \in \mathcal{B}_{0}}\right)$, e $\left(X,\left(\varphi_{b}\right)_{b \in \mathcal{B}_{0}}\right)$ um cone sobre $\mathcal{C}(c, F(-)): \mathcal{B} \rightarrow$ Set. Para cada $x \in X,\left(c,\left(\varphi_{b}(x)\right)_{b \in \mathcal{B}_{0}}\right)$ é um cone sobre $F$, portanto existe um único $\kappa(x): c \rightarrow l$ em $\mathcal{C}_{1}$ tal que $\psi_{b} \circ \kappa(x)=\varphi_{b}(x)$, para todo $b \in \mathcal{B}_{0}$. Assim, podemos definir $\kappa: X \rightarrow \mathcal{C}(c, l)$ tal que $\mathcal{C}\left(c, \psi_{b}\right) \circ \kappa=\varphi_{b}$, para todo $b \in \mathcal{B}_{0}$, e a unicidade decorre da unicidade de cada $\kappa(x)$. Logo, $\left(\mathcal{C}(c, l),\left(\mathcal{C}\left(c, \psi_{b}\right)\right)_{b \in \mathcal{B}_{0}}\right)$ é um limite de $\mathcal{C}(c, F(-))$.

Dualmente, o funtor hom contravariante $\mathcal{C}(-, c): \mathcal{C}^{o p} \rightarrow$ Set leva colimites de $\mathcal{C}$ em limites de Set.

Teorema 1.5.16. Sejam $\mathcal{A}, \mathcal{B}$ categorias pequenas, $\mathcal{C}$ uma categoria qualquer e $F: \mathcal{A} \rightarrow \mathcal{C}^{\mathcal{B}}$ um funtor. Se para cada $b \in \mathcal{B}_{0}$ o funtor $F(-)(b): \mathcal{A} \rightarrow \mathcal{C}$ possui um limite, então $F$ também possui um limite, que é computado ponto a ponto.

Denotando o limite do funtor $F(-)(b)$ por $\lim _{a \in \mathcal{A}_{0}}(F(a)(b))$ e o limite de $F$ por $\lim _{a \in \mathcal{A}_{0}} F(a)$, isso significa que

$$
\lim _{a \in \mathcal{A}_{0}}(F(a)(b))=\left(\lim _{a \in \mathcal{A}_{0}} F(a)\right)(b)
$$

Mais precisamente, lembrando que um limite de $F$ é um par $\left(l,\left(\varphi_{a}\right)_{a \in \mathcal{A}_{0}}\right)$, com $l \in\left(\mathcal{C}^{\mathcal{B}}\right)_{1}$, o lado direito representa $\left(l_{0}(b),\left(\left(\varphi_{a}\right)_{b}\right)_{a \in \mathcal{A}_{0}}\right)$. Ou seja, como a notação sugere, isto significa calcular $l$ em $b$ e considerar cada componente em $b$ de cada transformação natural $\varphi_{a}$.

Em outras palavras, podemos entender essa igualdade como: o valor do limite de $F$ calculado num objeto $b$ é o limite dos valores de $F(a)$ calculados em $b$. Como consequência imediata desse teorema, temos:

Corolário 1.5.17. Sejam $\mathcal{B}$ uma categoria pequena e $\mathcal{C}$ uma categoria completa. Então, a categoria $\mathcal{C}^{\mathcal{B}}$ é completa, e seus limites são computados ponto a ponto.

Dualmente, podemos obter resultados análogos para colimites.

Outro corolário, usando que os funtores hom preservam limites, é: 
Corolário 1.5.18. Seja $\mathcal{C}$ uma categoria pequena. O Funtor de Yoneda (covariante) $\mathcal{Y}_{\mathcal{C}}: \mathcal{C} \rightarrow$ Set $^{\mathcal{C}^{o p}}$ preserva limites.

Teorema 1.5.19. Sejam $\mathcal{C}$ uma categoria pequena e $F: \mathcal{C} \rightarrow$ Set um funtor. Então, $F$ pode ser apresentado como o colimite de funtores representáveis. ${ }^{10}$

Sendo um pouco mais preciso, esse teorema diz que $F$ é objeto do colimite de um funtor $\mathbf{F}: \mathcal{A} \rightarrow \operatorname{Set}^{\mathcal{C}}$ (para $\mathcal{A}$ uma categoria pequena) tal que $\mathbf{F}_{0}(a)$ é um funtor representável, para todo $a \in \mathcal{A}_{0}$. Ou seja, existem $c_{a} \in \mathcal{C}_{0}$ tais que:

$$
F=\operatorname{colim}_{a \in \mathcal{A}_{0}} \mathbf{F}(a) \cong \operatorname{colim}_{a \in \mathcal{A}} \mathcal{C}\left(c_{a},-\right)
$$

\subsection{Subobjetos}

A ideia dos subobjetos é, de certa forma, generalizar noções como subconjuntos e subgrupo; isto é, um objeto que "está dentro" de outro objeto na mesma categoria. Para definir esse conceito dentro da teorias das categorias, usamos monomorfismos:

Sejam $\mathcal{C}$ uma categoria e $a \in \mathcal{C}_{0}$. Defina $\operatorname{Mono}(a):=\left\{f \in \mathcal{C}_{1} \mid f\right.$ é $\operatorname{mono}$ e $\left.\operatorname{cod}(f)=a\right\}$ e a relação $f \preceq f^{\prime} \Leftrightarrow \exists g: x \rightarrow x^{\prime}\left(f^{\prime} \circ g=f\right)$, para $f: x \rightarrow a$ e $f^{\prime}: x^{\prime} \rightarrow a$ em $\operatorname{Mono}(a)$. É fácil ver que tal $g$ é mono (pois $f$ é mono, usando a proposição 1.2.3) e, quando existe, é única (pois $f^{\prime}$ é mono). Essa relação $\preceq$ é reflexiva e transitiva, e pode-se definir em $M o n o(a)$ a relação de equivalência $f \sim f^{\prime} \Leftrightarrow f \preceq f^{\prime}$ e $f^{\prime} \preceq f$ (ou, equivalentemente, se existe um iso $\kappa$ tal que $\left.f^{\prime} \circ \kappa=f\right)$. Fazendo o quociente, em $\frac{\operatorname{Mono}(a)}{\sim}$ definimos a relação de ordem $[f] \leq\left[f^{\prime}\right] \Leftrightarrow f \preceq f^{\prime}$.

Definição 1.6.1. Sejam $\mathcal{C}$ uma categoria e $a \in \mathcal{C}_{0}$. Considerando o que foi definido no parágrafo anterior, a família de subobjetos de $a$ é definida por $(\operatorname{Sub}(a), \leq):=\left(\frac{\operatorname{Mono}(a)}{\sim}, \leq\right)$. É comum dizer que um objeto $b \in \mathcal{C}_{0}$ é um subobjeto de $a$ se existe $f \in \mathcal{C}_{1}$ tal que $\operatorname{dom}(f)=b$ e $[f] \in \operatorname{Sub}(a)$.

Exemplo 1.6.2. Em Set, como esperado um conjunto $B$ é um subobjeto de um conjunto $A$ se $B \subseteq A$, basta considerar a inclusão $i: B \hookrightarrow A$. Talvez seja interessante mencionar, no entanto, que os subobjetos não traduzirão exatamente a noção de subconjunto em Set, e nem seria interessante sê-lo, pois subconjuntos não são invariantes por isomorfismos (que, em Set, são apenas funções bijetoras). De fato, pensando em conjuntos dentro de teoria das categorias, a

\footnotetext{
${ }^{10}$ Colocando de outra maneira, esse teorema diz que o Funtor de Yoneda $\mathcal{Y}_{\mathcal{C}^{o p}}: \mathcal{C}^{o p} \rightarrow$ Set $^{\mathcal{C}}$ é codenso (para mais detalhes, ver seção X.6 de [Lan98]).
} 
distinção entre, por exemplo, $\{0,1\}$ e $\{a, b\}$ (dois conjuntos com dois elementos) não é relevante: ambos possuem as mesmas propriedades categoriais. Porém, vale que todo mono $m: C \longmapsto A \mathrm{em}$ Set é equivalente a uma inclusão: mais especificamente à inclusão da imagem $m(C) \hookrightarrow A$.

Exemplo 1.6.3. Dadas duas caegorias $\mathcal{A}, \mathcal{B}$ e um funtor $F: \mathcal{A} \rightarrow \mathcal{B}$, um subfuntor de $F$ é um subobjeto de $F$ na categoria $\mathcal{B}^{\mathcal{A}}$ : um funtor $G: \mathcal{A} \rightarrow \mathcal{B}$ tal que existe uma transformação natural $\eta: G \Rightarrow F$ que é um mono (ou seja, cada $\eta_{a}$ é um mono). Para funtores $F: \mathcal{C}^{o p} \rightarrow$ Set, isso significa um funtor $G: \mathcal{C}^{o p} \rightarrow$ Set tal que $G_{0}(c) \subseteq F_{0}(c)$, para todo $c \in \mathcal{C}$, e para toda $f: c^{\prime} \rightarrow c$ em $\mathcal{C}_{1}, G_{1}(f)$ é a restrição de $F_{1}(f)$ a $G_{0}(c)$.

Agora, definamos a intersecção e a união de subobjetos, que serão importantes mais adiante quando estudarmos a lógica de topos.

Definição 1.6.4. Sejam $\mathcal{C}$ uma categoria, $a \in \mathcal{C}_{0}$ e $A=\left\{f_{i} \in M o n o(a) \mid i \in I\right\}$ uma família de monos com codomínio $a$ (indexada por um conjunto $I$ ). Chamando $[A]=\left\{\left[f_{i}\right] \in S u b(a) \mid i \in I\right\}$, temos:

1. a intersecção da família $A$ será o ínfimo de $[A] \operatorname{em~} S u b(a): \bigcap_{i \in I} f_{i}:=\bigwedge[A]$;

2. a união da família $A$ será o supremo de $[A] \operatorname{em} \operatorname{Sub}(a): \bigcup_{i \in I} f_{i}:=\bigvee[A]$

Observe que as operações estão bem definidas: dada outra família $B=\left\{g_{i} \in \operatorname{Mono}(a) \mid i \in\right.$ $I\}$ tal que $f_{i} \sim g_{i}$ para todo $i \in I$, então $[A]=[B]$. Logo, $\bigcap_{i \in I} f_{i}=\bigcap_{i \in I} g_{i}$ e $\bigcup_{i \in I} f_{i}=\bigcup_{i \in I} g_{i}$.

Se a categoria considerada tiver mais estrutura, será possível obter descrições explícitas da união e da intersecção de subobjetos. Faremos apenas o caso binário, pois é o que será relevante neste trabalho.

Teorema 1.6.5. Sejam $\mathcal{C}$ uma categoria com pullbacks binários, $a \in \mathcal{C}_{0}$, e $f: b \longmapsto a$ e $g: c \longmapsto a$ em $\mathcal{C}_{1}$. Então, a intersecção de $f$ e $g$ existe e $f \cap g=\left[f \circ g^{\prime}\right]$, a classe de equivalência da flecha obtida no pullback $\left(p, f^{\prime}, g^{\prime}\right)$ de $f$ e $g$.

Demonstração. Primeiro, observe que $f \circ g^{\prime}=g \circ f^{\prime}$ é, de fato, um mono (pois pullback de mono é mono), e é imediato da definição de $\preceq$ que $f \circ g^{\prime} \preceq f$ e $f \circ g^{\prime} \preceq g$. Agora, seja $h: d \longmapsto a$ tal que existem $h_{1}, h_{2}$ com $f \circ h_{1}=h$ e $g \circ h_{2}=h$. Pela definição do pullback, existe uma única 
flecha $\kappa: d \rightarrow p$ que torna o diagrama abaixo comutativo:

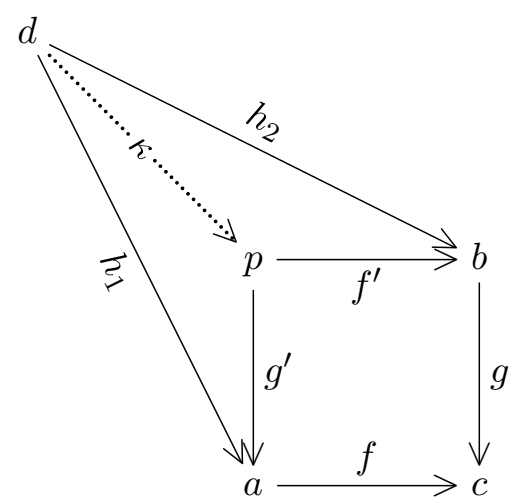

Logo, $h=f \circ h_{1}=\left(f \circ g^{\prime}\right) \circ \kappa$, e $h \preceq f \circ g^{\prime}$. Ou seja, $\left[f \circ g^{\prime}\right]$ é a maior das cotas inferiores, portanto o ínfimo.

Para intersecções finitas, precisaríamos considerar uma categoria com limites finitos, e a intersecção seria o limite da família de monos.

Teorema 1.6.6. Seja $\mathcal{C}$ uma categoria com coprodutos finitos e fatoração epi-mono. Sejam $a \in \mathcal{C}_{0}$ e $f: b \longmapsto a$ e $g: c \longmapsto a$ em $\mathcal{C}_{1}$. Considere a fatoração epi-mono im $([f, g]) \circ[f, g]^{\triangleright} d a$ flecha $[f, g]: b+c \rightarrow a$ do coproduto. Então, a união de $f$ e $g$ existe e $f \cup g=[i m([f, g])], a$ classe de equivalência da imagem de $[f, g]$.

Demonstração. Pela definição do coproduto de $f$ e $g$, temos que o diagrama abaixo é comutativo (onde já substituímos $[f, g]$ por sua fatoração epi-mono):

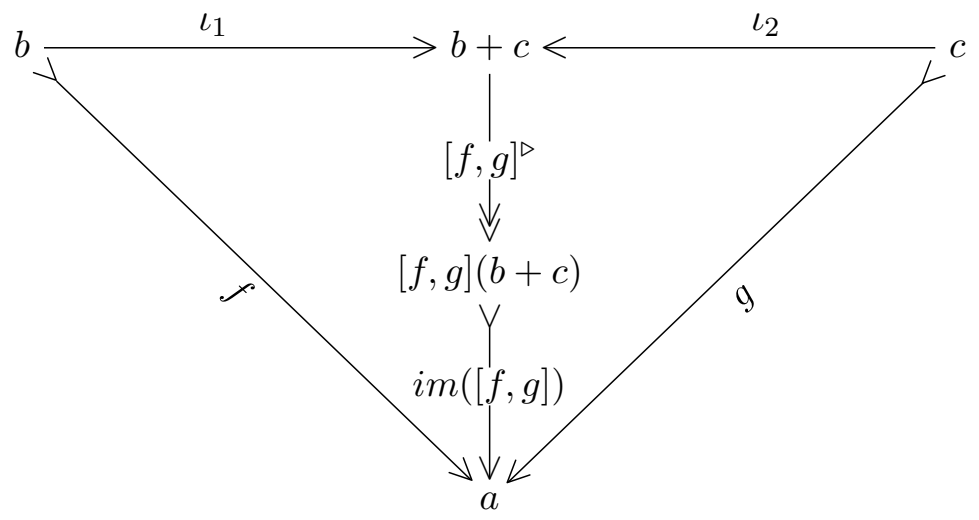

Isto é, $f=i m([f, g]) \circ\left([f, g]^{\triangleright} \circ \iota_{1}\right)$ e $g=i m([f, g]) \circ\left([f, g]^{\triangleright} \circ \iota_{2}\right)$, donde $f \preceq i m([f, g])$ e $g \preceq i m([f, g])$. Agora, seja $h: d \longmapsto a$ tal que existem $h_{1}, h_{2}$ com $f=h \circ h_{1}$ e $g=h \circ h_{2}$. Usando agora a definição do coproduto para $\left[h_{1}, h_{2}\right]$ e fazendo sua fatoração epi-mono, obtemos 
o seguinte diagrama comutativo:

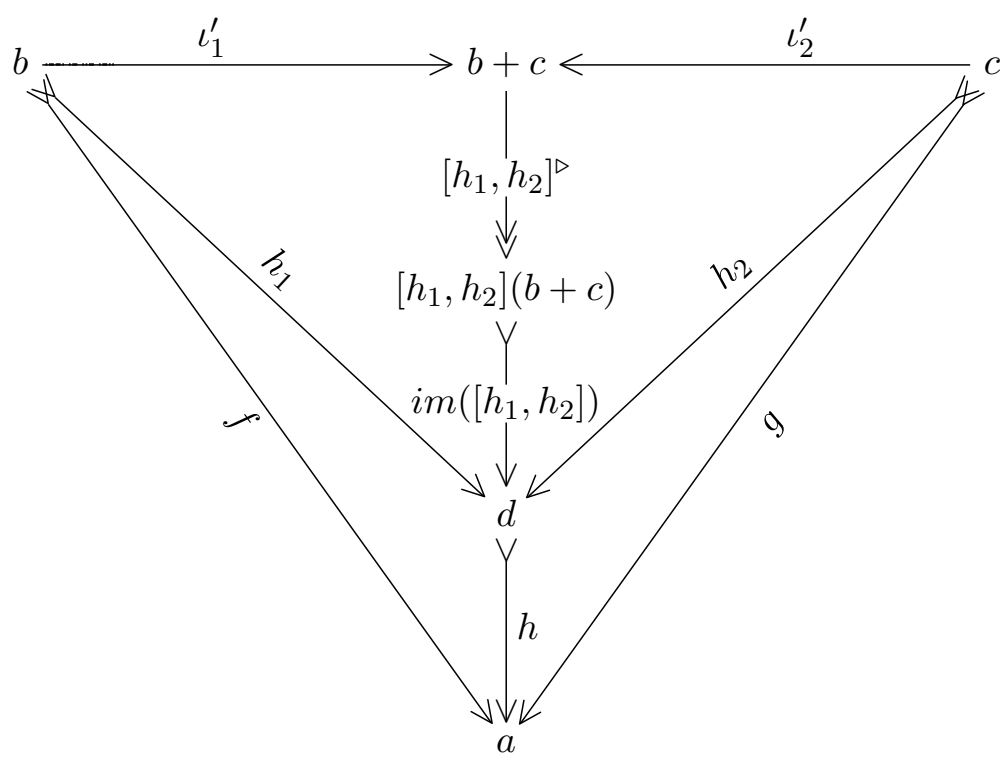

Assim, pela unicidade da definição do coproduto, vale $[f, g]=\left(h \circ \mathrm{im}\left(\left[h_{1}, h_{2}\right]\right)\right) \circ\left[h_{1}, h_{2}\right]^{\triangleright}$. Agora, pela unicidade a menos de isomorfismo da fatoração epi-mono de $[f, g]$, existe um único iso $\kappa:[f, g](b+c) \rightarrow\left[h_{1}, h_{2}\right](b+c)$ que torna o diagrama abaixo comutativo:

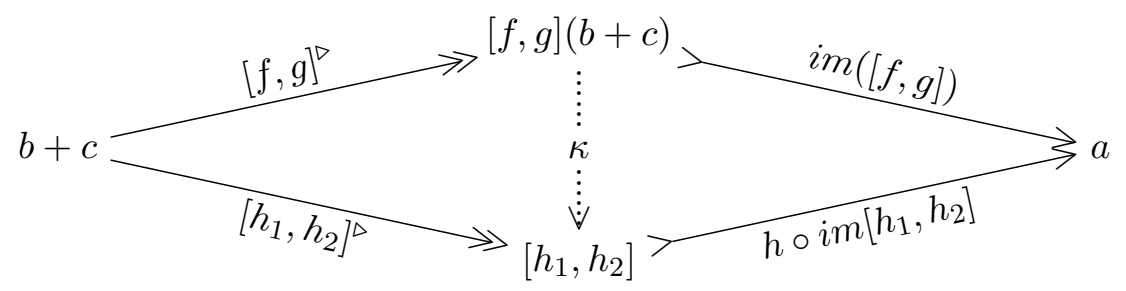

Dessa forma, $i m([f, g])=h \circ\left(i m\left(\left[h_{1}, h_{2}\right]\right) \circ \kappa\right)$, e $i m([f, g]) \preceq h$. Logo, $[i m([f, g])]$ é a menor das cotas superiores, isto é, o supremo.

A união finita consiste simplesmente em tomar a imagem do coproduto finito.

Exemplo 1.6.7. Em Set, essas operações correspondem à intersecção e união de subconjuntos. De fato, sejam $A, B \subseteq X$ com $i_{A}: A \hookrightarrow X$ e $i_{B}: B \hookrightarrow X$ as inclusões correspondentes. Assim, é fácil verificar que

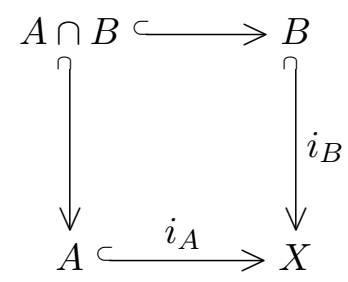

forma um pullback, pois $A \cap B=\left\{x \in X \mid i_{A}(x)=i_{B}(x)\right\}$. 
Agora, consideremos a união disjunta $A \sqcup B$ e a flecha coproduto $\left[i_{A}, i_{B}\right]: A \sqcup B \rightarrow X$, que leva $(a, 0) \mapsto a$ e $(b, 1) \mapsto b$. Fazendo sua fatoração epi-mono, $\left[i_{A}, i_{B}\right]^{\triangleright}$ é simplesmente a imagem direta, $\log \left[i_{A}, i_{B}\right](A \sqcup B)=A \cup B$, e $i m\left(\left[i_{A}, i_{B}\right]\right)$ é exatamente a inclusão $A \cup B \hookrightarrow X$.

Definição 1.6.8. Seja $\mathcal{C}$ uma categoria. Dizemos que $\mathcal{C}$ é bem potenciada se, para todo $a \in \mathcal{C}_{0}$, $S u b(a)$ é um conjunto.

Em categorias bem potenciadas com pullbacks, o mapa $a \mapsto S u b(a)$ define um funtor:

Definição 1.6.9. Seja $\mathcal{C}$ uma categoria bem potenciada que possui pullbacks. Definimos o funtor $S u b(-): \mathcal{C}^{o p} \rightarrow$ Set fazendo:

$$
S u b(-)_{0}(a):=\operatorname{Sub}(a), \text { para todo } a \in \mathcal{C}_{0}
$$

$$
\begin{aligned}
S u b(-)_{1}(f):=S u b(f): S u b(b) \rightarrow S u b(a), \text { para todo } f: a \rightarrow b \text { em } \mathcal{C}_{1}, \text { com } \\
\qquad S u b(f)([m]):=\left[m^{\prime}\right], \text { para todo }[m] \in S u b(b)
\end{aligned}
$$

onde $m^{\prime}$ é o pullback de $m$ ao longo de $f$ (e ser pullback garante que isso está bem definido).

\subsection{Adjunção}

Definição 1.7.1. Sejam $\mathcal{A}, \mathcal{B}$ categorias localmente pequenas. Uma adjunção de $\mathcal{A}$ em $\mathcal{B}$ é uma tripla $(L, R, \Phi)$, onde $L: \mathcal{B} \rightarrow \mathcal{A}$ e $R: \mathcal{A} \rightarrow \mathcal{B}$ são funtores e $\Phi: \mathcal{A}(L(-),-) \Rightarrow \mathcal{B}(-, R(-))$ é um isomorfismo natural. Nesse caso, dizemos que $L$ é um adjunto à esquerda de $R$ (e $R$ um adjunto à direita de $L$ ), e denotamos $L \dashv R$.

$\Phi$ ser um isomorfismo natural significa que, para cada $a \in \mathcal{A}_{0}$ e $b \in \mathcal{B}_{0}, \Phi_{a, b}: \mathcal{A}\left(L_{0}(b), a\right) \rightarrow$ $\mathcal{B}\left(b, R_{0}(a)\right)$ é uma bijeção "natural em $a$ e $b$ ", isto é, para todas flechas $f: a \rightarrow a^{\prime}$ em $\mathcal{A}_{1}$ e $g: b^{\prime} \rightarrow b$ em $\mathcal{B}_{1}^{o p}$, o seguinte diagrama é comutativo ${ }^{11}$ :

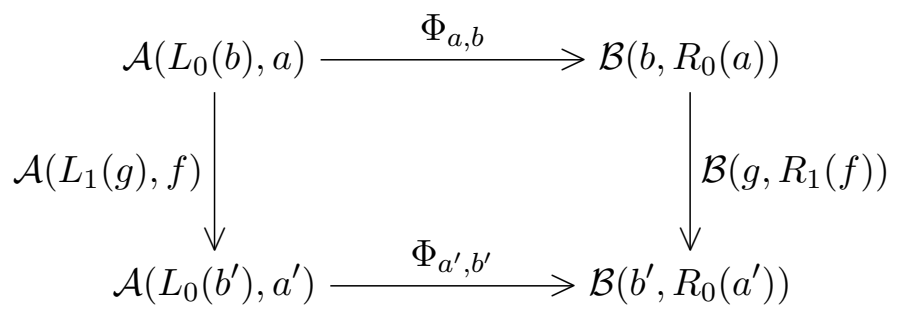

\footnotetext{
${ }^{11}$ Escrevemos $L_{1}(g)$ no diagrama para simplificar a notação, porém tecnicamente o mais correto seria $\left(L_{1}\left(g^{o p}\right)\right)^{o p}$ (para ser compatível com os domínios dos funtores).
} 
Todos os adjuntos à esquerda de um funtor são naturalmente isomorfos, e, analogamente, todos os adjuntos à direita de um funtor são naturalmente isomorfos.

Uma outra forma de definir adjunção é usando a unidade e a counidade da adjunção:

Definição 1.7.2. Sejam $\mathcal{A}, \mathcal{B}$ categorias localmente pequenas e $(L, R, \Phi)$ uma adjunção de $\mathcal{A}$ em $\mathcal{B}$. Com isso, definimos:

1. a unidade dessa adjunção como a transformação natural $\eta: i d_{\mathcal{B}} \Rightarrow R \circ L$ tal que, para todo $b \in \mathcal{B}_{0}, \eta_{b}:=\Phi_{L_{0}(b), b}\left(i d_{L_{0}(b)}\right): b \rightarrow R\left(L_{0}(b)\right)$;

2. a counidade dessa adjunção como a transformação natural $\theta: L \circ R \Rightarrow i d_{\mathcal{A}}$ tal que, para todo $a \in \mathcal{A}_{0}, \theta_{a}:=\Phi_{a, R_{0}(a)}\left(i d_{R_{0}(a)}\right): L\left(R_{0}(a)\right) \rightarrow a$.

Dessa forma, para cada $a \in \mathcal{A}$ e $b \in \mathcal{B}$, vale:

$$
\begin{gathered}
i d_{L}=\theta L \circ L \eta \\
i d_{R}=R \theta \circ \eta R
\end{gathered}
$$

A existência de transformações naturais $\eta: i d_{\mathcal{B}} \Rightarrow R \circ L$ e $\theta: L \circ R \Rightarrow i d_{\mathcal{A}}$ satisfazendo essas duas equações é equivalente a $L \dashv R$.

Exemplo 1.7.3. Alguns exemplos de adjunção:

- o funtor esquecimento $U:$ Mon $\rightarrow$ Set (que leva cada monoide a seu conjunto subjacente, e cada homomorfismo a ele mesmo, considerado como função apenas) possui um adjunto à esquerda $F$ : Set $\rightarrow$ Mon, o chamado funtor livre, que leva cada conjunto $X$ ao monoide livre sobre $X$;

- o funtor esquecimento $U: \mathbf{A b} \rightarrow \mathbf{G r p}$ possui como adjunto à esquerda o funtor abelianização $A: \operatorname{Grp} \rightarrow \mathbf{A b}:$ o funtor que leva cada grupo $G$ a $G /[G, G]$, o quociente de $G$ pelo seu subgrupo comutador;

- o funtor inclusão $I$ : CHaus $\rightarrow$ Top (que insere os espaços compactos Hausdorff e seus morfismos em Top) possui um adjunto à esquerda $\beta$ : Top $\rightarrow$ CHaus, que leva cada espaço topológico $X$ à sua compatificação de Stone-Čech $\beta X$;

- $\operatorname{sejam}(P, \leq)$ e $\left(P^{\prime}, \leq^{\prime}\right)$ conjuntos parcialmente ordenados e $L: P^{\prime} \rightarrow P$ e $R: P \rightarrow P^{\prime}$ funções decrescentes (ou seja, podemos considerá-las como funtores $L: P^{\prime} \rightarrow P^{o p}$ e $R$ : 
$\left.P^{o p} \rightarrow P^{\prime}\right)$. Então, $L \dashv R$ se, e somente se, $L$ e $R$ formam uma conexão de Galois; isto é, $p \leq L\left(p^{\prime}\right)$ se, e somente se, $p^{\prime} \leq^{\prime} R(p)$, para todos $p \in P$ e $p^{\prime} \in P^{\prime}$.

Proposição 1.7.4. Sejam $\mathcal{A}, \mathcal{B}, \mathcal{C}$ categorias e $L: \mathcal{B} \rightarrow \mathcal{A}, R: \mathcal{A} \rightarrow \mathcal{B}, L^{\prime}: \mathcal{C} \rightarrow \mathcal{B}, R^{\prime}: \mathcal{B} \rightarrow \mathcal{C}$ funtores tais que $L \dashv R$ e $L^{\prime} \dashv R^{\prime}$. Então, $L \circ L^{\prime} \dashv R^{\prime} \circ R$.

Isso é fácil usando as bijeções naturais entre os conjuntos hom:

$$
\mathcal{A}\left(\left(L \circ L^{\prime}\right)_{0}(c), a\right) \cong \mathcal{B}\left(L_{0}^{\prime}(c), R_{0}(a)\right) \cong \mathcal{C}\left(c,\left(R^{\prime} \circ R\right)_{0}(a)\right)
$$

para todos $a \in \mathcal{A}_{0}, b \in \mathcal{B}_{0}, c \in \mathcal{C}_{0}$.

Teorema 1.7.5. Sejam $\mathcal{A}, \mathcal{B}$ categorias e $R: \mathcal{A} \rightarrow \mathcal{B}$ e $L: \mathcal{B} \rightarrow \mathcal{A}$ funtores tais que $L \dashv R$. Então, $R$ preserva todos os limites em $\mathcal{A}$ e $L$ preserva todos os colimites em $\mathcal{B}$.

Façamos apenas um esboço, usando que o funtores hom preservam limites. Sejam $\mathcal{C}$ uma categoria pequena e $F: \mathcal{C} \rightarrow \mathcal{A}$ um funtor com limite que será denotado por $\lim _{c \in \mathcal{C}_{0}} F(c)$. Então:

$$
\begin{aligned}
\mathcal{B}\left(b, R_{0}\left(\lim _{c \in \mathcal{C}_{0}} F(c)\right)\right) \cong \mathcal{A}\left(L_{0}(b), \lim _{c \in \mathcal{C}_{0}} F(c)\right) & \cong \lim _{c \in \mathcal{C}_{0}} \mathcal{A}\left(L_{0}(b), F(c)\right) \cong \\
& \cong \lim _{c \in \mathcal{C}_{0}} \mathcal{B}\left(b, R_{0}(F(c))\right) \cong \mathcal{B}\left(b, \lim _{c \in \mathcal{C}_{0}}(R \circ F)(c)\right)
\end{aligned}
$$

Como as bijeções são naturais, pelo Lema de Yoneda (corolário 1.3.11) temos que

$$
R_{0}\left(\lim _{c \in \mathcal{C}_{0}} F(c)\right) \cong \lim _{c \in \mathcal{C}_{0}}(R \circ F)(c)
$$

como queríamos. O argumento para $L$ preservar colimites em $\mathcal{B}$ é análogo (e usa que o funtor hom contravariante leva colimites em limites).

Enunciemos, agora, dois teoremas importantes sobre a existência de funtores adjuntos.

Lema 1.7.6. Existência de objetos iniciais. Seja $\mathcal{C}$ uma categoria localmente pequena e pequena completa. Então, são equivalentes:

1. $\mathcal{C}$ possui objeto inicial;

2. $\mathcal{C}$ possui objeto inicial fraco, isto é, existe $\mathbf{0}_{w} \in \mathcal{C}_{0}$ tal que, para todo $c \in \mathcal{C}_{0}$, existe $h: \mathbf{0}_{w} \rightarrow c$ (ou seja, a flecha não precisa ser única);

3. $\mathcal{C}$ possui família pequena inicial fraca, isto é, existem um conjunto I e uma família $\left\{\mathbf{0}_{i} \in\right.$ $\left.\mathcal{C}_{0} \mid i \in I\right\}$ tais que, para todo $c \in \mathcal{C}_{0}$, existe $h: \mathbf{0}_{i} \rightarrow c$, para algum $i \in I$. 
Teorema 1.7.7. Teorema geral do funtor adjunto (Freyd). Sejam $\mathcal{A}, \mathcal{B}$ categorias, com $\mathcal{A}$ localmente pequena e pequena completa, e $F: \mathcal{A} \rightarrow \mathcal{B}$ um funtor que preserva limites pequenos. Então, são equivalentes:

1. F possui adjunto à esquerda;

2. condição conjunto solução: para todo $b \in \mathcal{B}_{0}$, existem um conjunto $I$ e uma família $\left\{f_{i}\right.$ : $b \rightarrow F_{0}\left(a_{i}\right)$ em $\left.\mathcal{B}_{1} \mid i \in I\right\}$ tais que toda flecha $g: b \rightarrow F_{0}(a)$ em $\mathcal{B}_{1}$ (para $a \in \mathcal{A}_{0}$ ) pode ser escrita como uma composição $g=F_{1}(h) \circ f_{i}$, para algum $i \in I$ e alguma $h: a_{i} \rightarrow$ a em $\mathcal{A}_{1}$.

Quando introduzirmos a categoria $a \downarrow F$ mais adiante, a condição conjunto solução será equivalente a $a \downarrow F$ possuir família pequena inicial fraca para todo $a \in \mathcal{A}_{0}$.

Lema 1.7.8. Existência de objetos iniciais (especial). Seja $\mathcal{C}$ uma categoria localmente pequena, pequena completa e com família pequena de cogeradores. Então, são equivalentes:

1. $\mathcal{C}$ possui objeto inicial;

2. para todo $c \in \mathcal{C}_{0}$, toda família de subobjetos de c possui intersecção.

Teorema 1.7.9. Teorema especial do funtor adjunto. Sejam $\mathcal{A}, \mathcal{B}$ categorias localmente pequenas e $F: \mathcal{A} \rightarrow \mathcal{B}$ um funtor que preserva limites pequenos. Suponha que $\mathcal{A}$ seja pequena completa, com família pequena de cogeradores, e que para todo a $\in \mathcal{A}_{0}$ o conjunto de subobjetos de a possua pullback (logo, intersecção). Então, são equivalentes:

1. F possui adjunto à esquerda;

2. F preserva pullbacks de famílias de monos.

Corolário 1.7.10. Sejam $\mathcal{A}, \mathcal{B}$ categorias localmente pequenas e $F: \mathcal{A} \rightarrow \mathcal{B}$ um funtor. Suponha que $\mathcal{A}$ seja pequeno completa, bem potenciada e possua família pequena de cogeradores. Então, $F$ possui adjunto à esquerda se, e somente se, $F$ preserva limites.

Esses resultados podem ser dualizados para obtermos condições para a existência de adjuntos à direita.

Finalmente, finalizamos a seção com uma caracterização de equivalências de categorias usando adjunção:

Proposição 1.7.11. Sejam $\mathcal{A}, \mathcal{B}$ categorias e $F: \mathcal{A} \rightarrow \mathcal{B}$ um funtor. Então, são equivalentes:

1. F é uma equivalência de categorias; 
2. F é pleno e fiel e possui um adjunto à esquerda pleno e fiel. ${ }^{12}$

\subsection{Categorias slice}

Categorias slice são essencialmente uma categoria de flechas sobre uma categoria. São caso particular de uma noção mais geral: a da categoria comma $F \downarrow G$ de dois funtores $F: \mathcal{A} \rightarrow \mathcal{C}$ e $G: \mathcal{B} \rightarrow \mathcal{C}$, onde todas as flechas (objetos nessa categoria) tem domínio na imagem de $F$ e codomínio na imagem de $G$. Porém, essa versão mais geral não será usada no presente trabalho, então apenas apresentamos o caso intermediário $F \downarrow c$ (quando $\mathcal{B}$ é a categoria terminal, com um objeto e um morfismo) e o caso especial da categoria slice $\mathcal{C} \downarrow c$ (quando $F=i d_{\mathcal{C}}$ ), além de suas versões duais.

Definição 1.8.1. Sejam $\mathcal{A}, \mathcal{B}$ categorias e $F: \mathcal{A} \rightarrow \mathcal{B}$ um funtor. Dado $b \in \mathcal{B}_{0}$, definimos a categoria $F \downarrow b$ que tem como objetos os pares $(a, f)$, com $a \in \mathcal{A}_{0}$ e $f: F_{0}(a) \rightarrow b$ em $\mathcal{B}_{1}$, e como flechas $\alpha:(a, f) \rightarrow\left(a^{\prime}, f^{\prime}\right)$ tais que $\alpha: a \rightarrow a^{\prime}$ é uma flecha em $\mathcal{A}_{1}$ e $f^{\prime} \circ F_{1}(\alpha)=f$, como no diagrama comutativo:

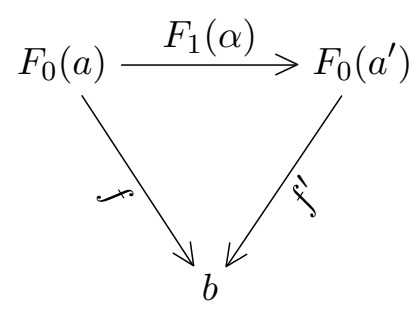

Analogamente, definimos a categoria $b \downarrow F$ que tem como objetos os pares $(a, f)$, com $a \in \mathcal{A}_{0}$ e $f: b \rightarrow F_{0}(a)$ em $\mathcal{B}_{1}$, e como flechas $\alpha:(a, f) \rightarrow\left(a^{\prime}, f^{\prime}\right)$ tais que $\alpha: a \rightarrow a^{\prime}$ é uma flecha em $\mathcal{A}_{1}$ e $F_{1}(\alpha) \circ f=f^{\prime}$, como no diagrama comutativo:

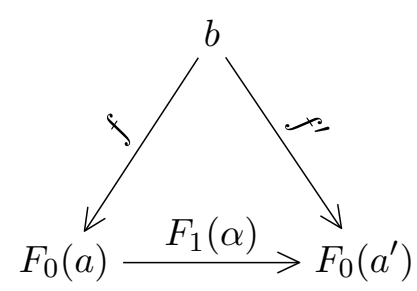

Essa definição nos permite definir adjunção de outra forma:

Proposição 1.8.2. Sejam $\mathcal{A}, \mathcal{B}$ categorias e $R: \mathcal{A} \rightarrow \mathcal{B}$ e $L: \mathcal{B} \rightarrow \mathcal{A}$ funtores. Então, são equivalentes:

\footnotetext{
${ }^{12}$ A implicação $(2 \Rightarrow 1)$ é estabelecida usando o axioma da escolha global em NBG.
} 
1. $L \dashv R$;

2. para todo $b \in \mathcal{B}_{0}$, existe uma transformação natural $\eta: i d_{\mathcal{B}} \Rightarrow R \circ L$ tal que $\left(L_{0}(b), \eta_{b}\right)$ é um objeto inicial em $b \downarrow R$;

3. para todo a $\in \mathcal{A}_{0}$, existe uma transformação natural $\theta: L \circ R \Rightarrow i d_{\mathcal{A}}$ tal que $\left(R_{0}(a), \theta_{a}\right)$ é um objeto terminal em $L \downarrow a$.

Definição 1.8.3. Sejam $\mathcal{C}$ uma categoria e $c \in \mathcal{C}_{0}$. A categoria slice $\mathcal{C} \downarrow c$ é definida tomando-se como objetos os pares $(a, f)$, com $a \in \mathcal{C}_{0}$ e $f: a \rightarrow c$ em $\mathcal{C}_{1}$, e como flechas $\alpha:(a, f) \rightarrow\left(a^{\prime}, f^{\prime}\right)$ tais que $\alpha: a \rightarrow a^{\prime}$ é uma flecha em $\mathcal{C}_{1}$ e $f^{\prime} \circ \alpha=f$, como no diagrama comutativo:

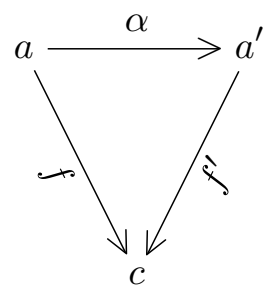

Analogamente, a categoria coslice $c \downarrow \downarrow C$ é definida tomando-se como objetos os pares $(a, f)$, com $a \in \mathcal{C}_{0}$ e $f: c \rightarrow a$ em $\mathcal{C}_{1}$, e como flechas $\alpha:(a, f) \rightarrow\left(a^{\prime}, f^{\prime}\right)$ tais que $\alpha: a \rightarrow a^{\prime}$ é uma flecha em $\mathcal{C}_{1}$ e $\alpha \circ f=f^{\prime}$, como no diagrama comutativo:

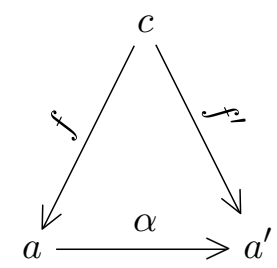

Observe que é fácil definir um funtor esquecimento $\Sigma_{c}: \mathcal{C} \downarrow c \rightarrow \mathcal{C}$, basta tomar $\left(\Sigma_{c}\right)_{0}(a, f):=$ $a$ e $\left(\Sigma_{c}\right)_{1}\left(\alpha:(a, f) \rightarrow\left(a^{\prime}, f^{\prime}\right)\right):=\alpha: a \rightarrow a^{\prime}$. Além disso, se $\mathcal{C}$ possui objeto inicial 0, então $\left(\mathbf{0}, \mathrm{O}_{c}\right)$ é inicial em $\mathcal{C} \downarrow c$, e $\mathcal{C} \downarrow c$ sempre possui objeto terminal: $\left(c, i d_{c}\right)$.

Exemplo 1.8.4. Para todo conjunto $I$, a categoria $\mathbf{S e t} \downarrow I$ pode ser pensada como a categoria dos conjuntos indexados por $I$. De fato, para cada objeto $(X, f)$ da categoria slice, podemos definir o conjunto $\left\{X_{i}=f^{-1}(\{i\}) \mid i \in I\right\}$; e para cada flecha $\alpha:(X, f) \rightarrow(Y, g)$, temos $f=g \circ \alpha$, de forma que para cada $i \in I$ podemos definir $\alpha_{i}: X_{i} \rightarrow Y_{i}$ como $\alpha_{i}(x)=\alpha(x)$.

Reciprocamente, dada uma família indexada $\left\{X_{i} \mid i \in I\right\}$, podemos definir $X:=\bigsqcup_{i \in I} X_{i}$ e $f: X \rightarrow I$ fazendo $f(x, i)=i$, para todo $(x, i) \in X$; e para cada $\beta_{i}: X_{i} \rightarrow Y_{i}$ (para outra família indexada $\left.\left\{Y_{i} \mid i \in I\right\}\right)$, podemos definir uma flecha $\beta:(X, f) \rightarrow(Y, g)$ fazendo $\beta(x, i):=\left(\beta_{i}(x), i\right)$, para todo $(x, i) \in X$, de forma que $f(x, i)=i=g\left(\beta_{i}(x), i\right)=g(\beta(x, i))$. 
Proposição 1.8.5. Sejam $\mathcal{C}$ uma categoria completa (resp. finitamente completa) e $c \in \mathcal{C}_{0}$. Então, $\mathcal{C} \downarrow c$ é uma categoria completa (resp. finitamente completa).

Proposição 1.8.6. Sejam $\mathcal{C}$ uma categoria cocompleta (resp. finitamente cocompleta) e $c \in \mathcal{C}_{0}$. Então, $\mathcal{C} \downarrow c$ é uma categoria cocompleta (resp. finitamente cocompleta).

Definição 1.8.7. Sejam $\mathcal{C}$ uma categoria com produtos binários e $c \in \mathcal{C}_{0}$. Definimos o funtor $c^{\star}: \mathcal{C} \rightarrow \mathcal{C} \downarrow c$ fazendo:

$$
c_{0}^{\star}(a):=\left(c \times a, \pi_{1}\right), \text { para todo } a \in \mathcal{C}_{0}, \text { onde }
$$

$\pi_{1}: c \times a \rightarrow c$ é a projeção na primeira coordenada

$$
c_{1}^{\star}(f):\left(c \times a, \pi_{1}\right) \rightarrow\left(c \times a^{\prime}, \pi_{1}^{\prime}\right), \text { para toda } f: a \rightarrow a^{\prime} \text { em } \mathcal{C}_{1}, \operatorname{com} c_{1}^{\star}(f)=i d_{c} \times f
$$

Teorema 1.8.8. Sejam $\mathcal{C}$ uma categoria localmente pequena com produtos binários e $c \in \mathcal{C}_{0}$. Então, temos a adjunção $\Sigma_{c} \dashv c^{\star}$.

Demonstração. Primeiramente, para cada $a \in \mathcal{C}_{0}$ e $(b, g) \in(\mathcal{C} \downarrow c)_{0}$, temos $\left(\Sigma_{c}\right)_{0}(b, g)=b$ e $c_{0}^{\star}(a)=\left(c \times a, \pi_{1}\right)$, e definimos o isomorfismo $\Phi_{a,(b, g)}: \mathcal{C}(b, a) \rightarrow \mathcal{C} \downarrow c\left((b, g),\left(c \times a, \pi_{1}\right)\right)$ fazendo:

$$
\Phi_{a,(b, g)}(f):=\langle g, f\rangle:(b, g) \rightarrow\left(c \times a, \pi_{1}\right), \text { para toda } f: b \rightarrow a \text { em } \mathcal{C}_{1}
$$

É fácil verificar que isso é, de fato, uma bijeção: dada $\alpha:(b, g) \rightarrow\left(c \times a, \pi_{1}\right)$, defina $h=\pi_{2} \circ \alpha$ : $b \rightarrow a$ (para $\pi_{2}: c \times a \rightarrow a$ a projeção na segunda coordenada). Assim, $\pi_{1} \circ \alpha=g$ e $\pi_{2} \circ \alpha=h, \mathrm{e}$ pela unicidade da flecha do produto, $\alpha=\langle g, h\rangle=\Phi_{a,(b, g)}(h)$. Além disso, se $h, h^{\prime}: b \rightarrow a$ em $\mathcal{C}_{1}$ são tais que $\Phi_{a,(b, g)}(h)=\Phi_{a,(b, g)}\left(h^{\prime}\right)$, então $\langle g, h\rangle=\left\langle g, h^{\prime}\right\rangle$, e $h=\pi_{2} \circ\langle g, h\rangle=\pi_{2} \circ\left\langle g, h^{\prime}\right\rangle=h^{\prime}$.

Agora, sejam $h: a \rightarrow a^{\prime}$ em $\mathcal{C}_{1}$ e $\beta:\left(b^{\prime}, g^{\prime}\right) \rightarrow(b, g)$ em $(\mathcal{C} \downarrow c)_{1}^{o p}$, de forma que $\Sigma_{c}(\beta)=\beta$ : $b^{\prime} \rightarrow b$ e $c^{\star}(h)=i d_{c} \times h:\left(c \times a, \pi_{1}\right) \rightarrow\left(c \times a^{\prime}, \pi_{1}^{\prime}\right)$. Com isso, temos as funções:

$$
\mathcal{C}(\beta, h): \mathcal{C}(b, a) \rightarrow\left(b^{\prime}, a^{\prime}\right) \text { tal que } f \mapsto h \circ f \circ \beta
$$

$\mathcal{C} \downarrow c\left(\beta, i d_{c} \times h\right): \mathcal{C} \downarrow c\left((b, g),\left(c \times a, \pi_{1}\right)\right) \rightarrow \mathcal{C} \downarrow c\left(\left(b^{\prime}, g^{\prime}\right),\left(c \times a^{\prime}, \pi_{1}^{\prime}\right)\right)$ tal que $\alpha \mapsto\left(i d_{c} \times h\right) \circ \alpha \circ \beta$ Agora, seja $f: b \rightarrow a$ em $\mathcal{C}_{1}$. Então, tendo em mente o diagrama da definição de adjunção,

$$
\left(\mathcal{C} \downarrow c\left(\beta, i d_{c} \times h\right) \circ \Phi_{a,(b, g)}\right)(f)=\left(i d_{c} \times h\right) \circ\langle g, f\rangle \circ \beta=\left\langle g^{\prime}, h \circ f \circ \beta\right\rangle=\left(\Phi_{a^{\prime},\left(b^{\prime}, g^{\prime}\right)} \circ \mathcal{C}(\beta, h)\right)(f)
$$

Ou seja, mostramos a naturalidade de $\Phi$, donde podemos concluir que $\Sigma_{c} \dashv c^{\star}$. 
Observe que produtos binários na categoria slice são pullbacks na categoria original. De fato, sejam $(a, f),(b, g) \in(\mathcal{C} \downarrow c)_{0}$ e considere o pullback de $f$ por $g$ :

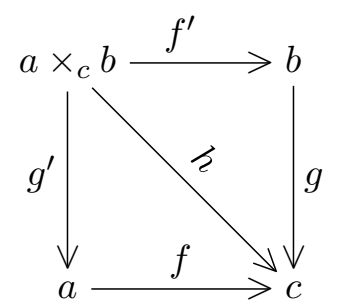

Com isso, para todas flechas $\alpha:(d, k) \rightarrow(a, f), \beta:(d, k) \rightarrow(g, b)$ em $(\mathcal{C} \downarrow c)_{1}$, temos $f \circ \alpha=k=$ $g \circ \beta$. Portanto, pelo pullback, existe uma única $\kappa: d \rightarrow c$ tal que $g^{\prime} \circ \kappa=\alpha$ e $f^{\prime} \circ \kappa=\beta$, donde $h \circ \kappa=k$. Ou seja, $\kappa$ define uma flecha única $(d, k) \rightarrow\left(a \times_{c} b, h\right)$ :

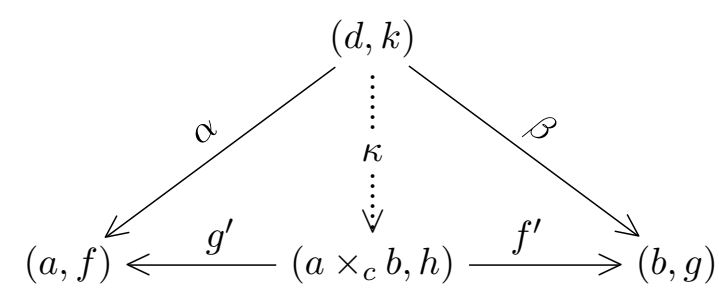

e $\left(\left(a \times_{c} b, h\right), g^{\prime}, f^{\prime}\right)$ é o produto de $(a, f)$ e $(b, g)$.

Dessa forma, podemos considerar um caso especial do funtor $c^{\star}$ acima quando tomamos como base uma categoria slice. Primeiramente, note que dado $(a, f) \in(\mathcal{C} \downarrow c)_{0}$ é fácil construir um isomorfismo entre $(\mathcal{C} \downarrow c) \downarrow(a, f)$ e $\mathcal{C} \downarrow a$, basta fazer:

$$
((b, g), \beta) \quad \mapsto \quad(b, \beta) \quad \mapsto \quad((b, f \circ \beta), \beta)
$$

Nesse caso, o funtor esquecimento $\Sigma_{f}:(\mathcal{C} \downarrow c) \downarrow(a, f) \cong \mathcal{C} \downarrow a \rightarrow \mathcal{C} \downarrow c$ será dado por precomposição $\operatorname{com} f$ :

$$
\left(\Sigma_{f}\right)_{0}(b, g)=(b, f \circ g), \text { para todo }(b, g) \in(\mathcal{C} \downarrow a)_{0}
$$

$$
\left(\Sigma_{f}\right)_{1}(\beta)=\beta:(b, f \circ g) \rightarrow(d, f \circ h), \text { para toda } \beta:(b, g) \rightarrow(d, h) \text { em }(\mathcal{C} \downarrow a)_{1}
$$

Assim, dado $(a, f) \in(\mathcal{C} \downarrow c)_{0}$, o funtor $f^{\star}: \mathcal{C} \downarrow c \rightarrow(\mathcal{C} \downarrow c) \downarrow(a, f) \cong \mathcal{C} \downarrow a$ levará $(b, g)$ ao pullback $g^{\prime}$ de $g$ ao longo de $f$. Para as flechas, dada $\beta:(b, g) \rightarrow(d, h)$, temos $h \circ \beta=g$. Fazendo o pullback $\left(b \times_{c} a, f_{g}^{\prime}, g^{\prime}\right)$ de $f$ e $g$, temos $f \circ g^{\prime}=g \circ f_{g}^{\prime}=h \circ \beta \circ f_{g}^{\prime}$. Dessa forma, fazendo agora o pullback $\left(d \times_{c} a, f_{h}^{\prime}, h^{\prime}\right)$ de $f$ e $h$, temos que existe uma única $\kappa: b \times_{c} a \rightarrow d \times_{c} a$ fazendo o 
diagrama abaixo comutar:

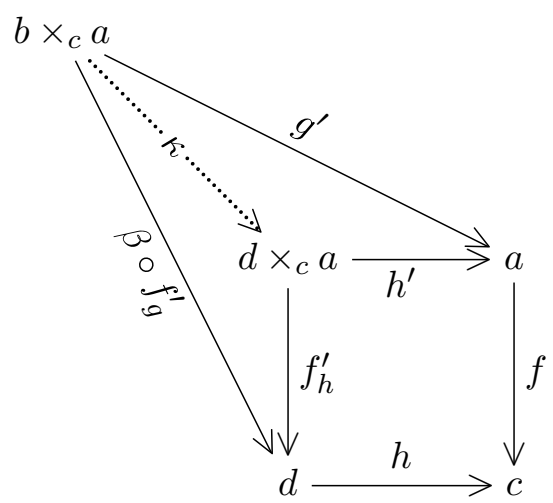

donde $h^{\prime} \circ \kappa=g^{\prime}$, e $\kappa$ define uma flecha $\left(b \times_{c} a, g^{\prime}\right) \rightarrow\left(d \times_{c} a, h^{\prime}\right)$ em $(\mathcal{C} \downarrow a)_{1}$.

Com isso, podemos definir o funtor $f^{\star}$ de forma mais direta:

Definição 1.8.9. Sejam $\mathcal{C}$ uma categoria com pullbacks e $f: a \rightarrow c$ em $\mathcal{C}_{1}$. Definimos o funtor $f^{\star}: \mathcal{C} \downarrow c \rightarrow \mathcal{C} \downarrow a$ fazendo:

$$
f_{0}^{\star}(b, g):=\left(b \times_{c} a, g^{\prime}\right), \text { para todo }(b, g) \in(\mathcal{C} \downarrow c)_{0}
$$

$f_{1}^{\star}(\beta):\left(b \times_{c} a, g^{\prime}\right) \rightarrow\left(d \times_{c} a, h^{\prime}\right)$, para toda $\beta:(b, g) \rightarrow(d, h)$ em $(\mathcal{C} \downarrow c)_{1}, \operatorname{com} f_{1}^{\star}(\beta):=\kappa$

onde $g^{\prime}$ e $h^{\prime}$ são os pullbacks de $g$ e $h$ ao longo de $f$ (respectivamente), e com $\kappa: b \times{ }_{c} a \rightarrow d \times{ }_{c} a$ obtida como nos comentários acima.

Como caso particular do teorema anterior, temos novamente uma adjunção, usando agora as descrições mais simples de $\Sigma_{f}$ e $f^{\star}$.

Proposição 1.8.10. Sejam $\mathcal{C}$ uma categoria localmente pequena com pullbacks e $f: a \rightarrow c$ em $\mathcal{C}_{1}$. Então, temos a adjunção $\Sigma_{f} \dashv f^{\star}$.

\subsection{Objeto números naturais}

Para finalizar o capítulo, introduzimos uma formulação categorial para o axioma do infinito. Tal definição faz sentido num contexto bem geral, mas é num topos que será possível mostrar como os objetos números naturais se comportam de forma semelhante a $\mathbb{N}$ em Set.

Definição 1.9.1. Seja $\mathcal{C}$ uma categoria com objeto terminal 1. Um objeto números naturais em $\mathcal{C}$ é uma tripla $\left(\mathbb{N}_{\mathcal{C}}, 0_{\mathcal{C}}, s\right)$, onde $\mathbb{N}_{\mathcal{C}} \in \mathcal{C}_{0}$ e $0_{\mathcal{C}}: \mathbf{1} \rightarrow \mathbb{N}_{\mathcal{C}}, s: \mathbb{N}_{\mathcal{C}} \rightarrow \mathbb{N}_{\mathcal{C}}$ são flechas em $\mathcal{C}_{1}$, tal 
que, para toda outra tripla $(M, m, \sigma) \operatorname{com} M \in \mathcal{C}_{0}$ e $m: \mathbf{1} \rightarrow M, \sigma: M \rightarrow M$ em $\mathcal{C}_{1}$, existe uma única $\mu: \mathbb{N}_{\mathcal{C}} \rightarrow M \in \mathcal{C}_{1}$ que torna o diagrama abaixo comutativo:

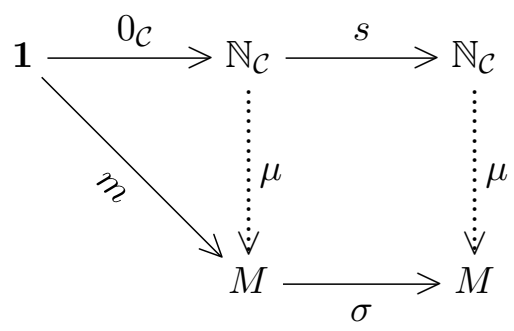

É fácil ver que, se uma categoria admite objeto números naturais, então é único a menos de isomorfismo.

Exemplo 1.9.2. O fato de os números naturais em Set constituírem um objeto números naturais é exatamente o teorema da recursão: dado $a \in M$ (isso equivale a considerar a função $m:\{*\} \rightarrow$ $M \operatorname{com} m(*)=a$ ) e uma função $\sigma: M \rightarrow M$, por recursão existe uma única função $\mu: \mathbb{N} \rightarrow M$ tal que $\mu(0)=a$ e $\mu(n+1)=\sigma(\mu(n))$, o que corresponde precisamente à comutatividade do diagrama da definição de objeto números naturais.

Aliás, o teorema da recursão é frequentemente introduzido como uma consequência dos axiomas de Peano, porém pode-se mostrar a recíproca também vale: o teorema da recursão implica nos axiomas de Peano ${ }^{13}$. Veremos, mais adiante, que num topos também vale uma equivalência semelhante entre duas noções de números naturais.

${ }^{13}$ Ver seção 11 do segundo capítulo de [BL67] para a demonstração desse fato 


\section{Capítulo 2}

\section{Topos de Grothendieck}

Neste capítulo, apresentamos a noção "original" de topos: como uma categoria de feixes de conjuntos, começando com feixes sobre espaços topológicos. Então, introduzimos locales, uma forma de "topologia sem pontos" que retém estrutura suficiente para definir feixes sem precisar de um espaço topológico usual, e mostramos como as construções feitas para espaços topológicos podem ser adaptadas para locales. Além disso, apresentamos brevemente os funtores que relacionam a categorias dos espaços topológicos e a categoria dos locales. Ao final, generalizamos a noção de feixe para feixes sobre sítios (usando uma noção de cobertura chamada de topologia de Grothendieck), para, enfim, podermos definir um topos de Grothendieck.

A ideia aqui será apresentar uma visão geral do assunto, por isso não nos alongamos muito nos aspectos mais técnicos. Uma exposição mais detalhada é feita em [Bor08c] (capítulos 1 a 3), [Joh02b] (capítulos C1 e C2) e [LM94] (capítulos II e III).

\subsection{Feixes sobre espaços topológicos}

Intuitivamente, um feixe (sobre um espaço topológico) é uma maneira de "atribuir dados" a um espaço topológico de forma que os dados atribuídos (de modo "compatível") para cada cobertura de um aberto possam ser "colados" para o aberto inteiro em questão. Em certo sentido, feixes fornecem uma "tradução" entre dados definidos localmente e dados definidos globalmente.

Há duas maneiras de construir a categoria de feixes sobre um espaço topológico, uma com feixes "geométricos" (que usa homeomorfismos locais) e outra com feixes "funtoriais" (que usa funtores da forma $F: \mathcal{O}(X)^{o p} \rightarrow$ Set, onde $\mathcal{O}(X)$ denota o reticulado dos abertos de um espaço 
topológico $X)$. Comecemos com os feixes geométricos, cuja definição é menos abstrata, embora os feixes funtoriais ilustrem de forma mais clara a intuição descrita acima.

Definição 2.1.1. Sejam $(X, \mathcal{O}(X))$ e $(Y, \mathcal{O}(Y))$ espaços topológicos. Uma função $f: X \rightarrow Y$ é dita um homeomorfismo local (de espaços topológicos) se, para todo $x \in X$, existem abertos $U \in \mathcal{O}(X), \operatorname{com} x \in U$, e $V \in \mathcal{O}(Y), \operatorname{com} f(x) \in V$, tais que a restrição $f_{\mid U}: U \rightarrow V$ é um homeomorfismo.

Observe que todo homeomorfismo local é uma função contínua e aberta.

Definição 2.1.2. Sejam $(X, \mathcal{O}(X))$ e $(Y, \mathcal{O}(Y))$ espaços topológicos. Um feixe geométrico sobre $X$ é um homeomorfismo local $f: Y \rightarrow X$. Como composição de homeomorfismos locais também é um homeomorfismo local, podemos definir a categoria $\mathbf{L H}_{t o p}$ dos espaços topológicos e homeomorfismos locais como flechas. A categoria slice $\mathbf{L H}_{t o p} \downarrow X$ é chamada de categoria dos feixes geométricos sobre $X$.

Exemplo 2.1.3. É claro que todo homeomorfismo também é um homeomorfismo local. Seja $(X, \mathcal{O}(X))$ um espaço topológico e $U \in \mathcal{O}(X)$ com a topologia induzida. Então, a inclusão $i_{U}: U \hookrightarrow X$ é um homeomorfismo local.

Exemplo 2.1.4. Seja $(Y, \mathcal{O}(Y))$ um espaço topológico e $(K, \mathcal{O}(K))$ um espaço munido da topologia discreta. Então, $\pi_{2}: K \times Y \rightarrow Y$, a projeção na segunda coordenada, é um homeomorfismo local: dado $(k, y) \in K \times Y$, temos $\{k\} \in \mathcal{O}(K)$ e, para toda vizinhança aberta $V \in \mathcal{O}(Y)$ de $\pi_{2}(k, y)=y$, a restrição $\pi_{2 \mid\{a\} \times V}:\{a\} \times V \rightarrow V$ é homeomorfismo.

Definição 2.1.5. Sejam $(X, \mathcal{O}(X))$ e $(Y, \mathcal{O}(Y))$ espaços topológicos e $f: Y \rightarrow X$ contínua. A fibra de $f$ num ponto $p \in X$ é o conjunto $X_{p}:=f^{-1}(\{p\})$.

Definição 2.1.6. Sejam $(X, \mathcal{O}(X))$ e $(Y, \mathcal{O}(Y))$ espaços topológicos e $f: X \rightarrow Y$ contínua. Uma seção contínua de $f$ sobre $V \in \mathcal{O}(Y)$ é uma função contínua $s: V \rightarrow X$ tal que $f \circ s=i_{V}$, onde $i_{V}: V \hookrightarrow Y$ é a inclusão. Denotamos por $\Gamma(f)(V)$ o conjunto das seções contínuas de $f$ sobre $V$.

Se $f: X \rightarrow Y$ for um homeomorfismo local, então as seções contínuas de $f$ sobre $V \in \mathcal{O}(Y)$ são funções abertas, e o conjunto

$$
\left\{s(V) \mid s \in \bigcup_{V \in \mathcal{O}(V)} \Gamma(f)(V)\right\} \subseteq \mathcal{O}(X)
$$

forma uma base para a topologia de $X$. 
Definição 2.1.7. Seja $(X, \mathcal{O}(X))$ um espaço topológico. Um prefeixe de conjuntos ${ }^{1}$ sobre $X$ é um funtor $F: \mathcal{O}(X)^{o p} \rightarrow$ Set (considerando $\mathcal{O}(X)$ como uma categoria de preordem, ver exemplo 1.1.3). Para cada $U \in \mathcal{O}(X)$, chamamos $F_{0}(U)$ de o conjunto das seções de $F$ sobre $U$. Para cada inclusão $i_{V, U}: V \hookrightarrow U$ em $\mathcal{O}(X)$, a função $F_{1}\left(i_{V, U}\right): F_{0}(U) \rightarrow F_{0}(V)$ é chamada de restrição e, para cada $s \in F_{0}(U)$, denotamos $\left.s\right|_{V} ^{U}:=F_{1}\left(i_{V, U}\right)(s)$. $\operatorname{Set}^{\mathcal{O}(X)^{o p}}$ é a categoria dos prefeixes funtoriais (em $X$ ) e das transformações naturais entre eles.

Observe que, pela funtorialidade de $F$, vale que $F_{1}\left(i_{U, U}\right)=i d_{F(U)}$ e, para $W \subseteq V \subseteq U$, temos $F_{1}\left(i_{W, V}\right) \circ F_{1}\left(i_{V, U}\right)=F_{1}\left(i_{W, U}\right)$, ou seja, para todo $s \in F_{0}(U),\left.\left.s\right|_{W} ^{V} \circ s\right|_{V} ^{U}=\left.s\right|_{W} ^{U}$.

Definição 2.1.8. Sejam $(X, \mathcal{O}(X))$ um espaço topológico e $F: \mathcal{O}(X)^{o p} \rightarrow$ Set um prefeixe. $F$ é dito um feixe (funtorial) de conjuntos sobre $X$ se, para todo $U \in \mathcal{O}(X)$, para toda cobertura $\left\{U_{i} \in \mathcal{O}(X) \mid i \in I\right\}$ de $U$ e para toda família $F$-compatível $\left\{s_{i} \in F_{0}\left(U_{i}\right) \mid i \in I\right\}$ (isto é, para todos $\left.i, j \in I,\left.s_{i}\right|_{U_{i} \cap U_{j}} ^{U_{i}}=\left.s_{j}\right|_{U_{i} \cap U_{j}} ^{U_{j}}\right)$, existe um único $s \in F_{0}(U)$ tal que $\left.s\right|_{U_{i}} ^{U}=s_{i}$, para todo $i \in I$. Em outras palavras, toda família compatível admite uma única colagem. A categoria dos feixes e transformações naturais entre eles será denotada por $\mathbf{S h}(X)$.

Há uma forma mais concisa de definir um feixe usando equalizadores:

Proposição 2.1.9. Sejam $(X, \mathcal{O}(X))$ um espaço topológico e $F: \mathcal{O}(X)^{o p} \rightarrow$ Set um prefeixe. Então, são equivalentes:

1. Fé feixe;

2. para todo $U \in \mathcal{O}(X)$ e para toda cobertura $\left\{U_{i} \in \mathcal{O}(X) \mid i \in I\right\}$ de $U$,

$$
F_{0}(U) \stackrel{d}{\longrightarrow} \prod_{i \in I} F_{0}\left(U_{i}\right) \stackrel{r}{\longrightarrow} \prod_{(i, j) \in I \times I} F_{0}\left(U_{i} \cap U_{j}\right)
$$

é um diagrama de equalizador, onde:

$$
\begin{gathered}
d(s)=\left(\left.s\right|_{U_{i}} ^{U}\right)_{i \in I} \\
r\left(\left(s_{i}\right)_{i \in I}\right)=\left(\left.s_{j}\right|_{U_{i} \cap U_{j}} ^{U_{j}}\right)_{(i, j) \in I \times I} \\
e\left(\left(s_{i}\right)_{i \in I}\right)=\left(\left.s_{i}\right|_{U_{i} \cap U_{j}} ^{U_{i}}\right)_{(i, j) \in I \times I}
\end{gathered}
$$

\footnotetext{
${ }^{1}$ Seria possível definir um prefeixe sobre uma categoria qualquer. Inclusive, historicamente aplicações iniciais de feixes usavam categorias algébricas, como Ab (dos grupos abelianos) ou cRing (dos anéis comutativos com unidade). Porém, para o desenvolvimento da teoria de topos, focaremos em prefeixes de conjuntos.
} 
Exemplo 2.1.10. Como exemplo de feixe, considere o funtor $C(-, \mathbb{R}): \mathcal{O}(X)^{o p} \rightarrow$ Set que leva cada aberto $U$ de $X$ ao conjunto das funções contínuas $C(U, \mathbb{R})$. Dada $f \in C(U, \mathbb{R})$ e $V \subseteq U$, $f \uparrow_{V}^{U}: V \rightarrow \mathbb{R}$ é apenas a restrição da função $f$ a $V$. Com isso, toda família compatível de funções $f_{i} \in C\left(U_{i}, \mathbb{R}\right)$, com $\left\{U_{i} \in \mathcal{O}(X) \mid i \in I\right\}$ cobertura de $U$, existe uma única função $f$ tal que $f \uparrow_{U_{i}}^{U}=f_{i}$; basta tomar $f(x)=f_{i}(x)$, para cada $x \in U_{i}, i \in I .^{2}$

Definição 2.1.11. Sejam $(X, \mathcal{O}(X))$ um espaço topológico e $F: \mathcal{O}(X) \rightarrow$ Set um prefeixe. A fibra de $F$ num ponto $x \in X$ é

$$
F_{x}:=\frac{\coprod\left\{F_{0}(U) \mid U \in \mathcal{O}(X), x \in U\right\}}{\approx_{x}}
$$

onde $\approx_{x}$ é a relação de equivalência $(r, U) \approx_{x}(s, V) \Leftrightarrow$ existe $W \in \mathcal{O}(X)$ com $x \in W$ e $W \subseteq U, V$ tal que $r \uparrow_{W}^{U}=s \uparrow_{W}^{V}{ }^{3}$

Podemos, enfim, começar a construir os funtores que relacionarão as categorias de feixes geométricos e feixes funtoriais.

Proposição 2.1.12. Seja $(X, \mathcal{O}(X))$ um espaço topológico. Então, $\Gamma: \operatorname{Top} \downarrow X \rightarrow \operatorname{Set}^{\mathcal{O}(X)^{o p}}$ é um funtor, onde, para todo $f \in(\mathbf{T o p} \downarrow X)_{0}, \Gamma_{0}(f): \mathcal{O}(X)^{o p} \rightarrow$ Set é o funtor que leva cada $U \in L$ ao conjunto $\Gamma_{0}(f)(U)$ das seções contínuas de $f$ sobre $X$ (e agindo nas flechas por precomposição). Além disso, a imagem de $\Gamma$ está em $\mathbf{S h}(X)$.

É fácil ver que $F=\Gamma_{0}(f)$ é feixe funtorial: para cada $U \in \mathcal{O}(X),\left\{U_{i} \in \mathcal{O}(X) \mid i \in I\right\}$ cobertura de $U$ e $\left\{s_{i} \in F_{0}\left(U_{i}\right) \mid i \in I\right\}$ família $F$-compatível, basta definir $s: U \rightarrow X$ fazendo, para cada $x \in X$, existe $i \in I$ tal que $x \in U_{i}$, e $s(x)=s_{i}(x)$. A família ser compatível garante que está bem definido e garante a unicidade. $s$ ser uma seção contínua de $f$ sobre $U$ decorre do fato de cada $s_{i}$ o ser (sobre cada aberto da cobertura).

Agora, seja $F: \mathcal{O}(X)^{o p} \rightarrow$ Set um prefeixe. Podemos definir um espaço topológico fazendo $E^{(F)}:=\coprod_{x \in X} F_{x}$. Dado $U \in \mathcal{O}(X)$, para toda seção $s \in F_{0}(U)$, definimos $\hat{s}: U \rightarrow E^{(F)}$ fazendo $\hat{s}(a)=\left(s_{a}, a\right) \in F_{a} \times\{a\}$ (onde $s_{a}:=[(s, U)]_{a}$ denota a classe de equivalência). Com isso, podemos munir $E^{(F)}$ da menor topologia que contém $\left\{\hat{s}(U) \subseteq E^{(F)} \mid U \in \mathcal{O}(X), s \in F_{0}(U)\right\}$.

Proposição 2.1.13. Seja $(X, \mathcal{O}(X))$ um espaço topológico. Então, $\Theta: \operatorname{Set}^{\mathcal{O}(X)^{o p}} \rightarrow \operatorname{Top} \downarrow X$ é um funtor, onde, para todo $F \in\left(\operatorname{Set}^{\mathcal{O}(X)^{o p}}\right)_{0}, \Theta_{0}(F): E^{(F)} \rightarrow X$ é tal que $\Theta(F)\left(s_{x}, x\right)=x$, para todo $x \in X$. Além disso, a imagem de $\Theta$ está em $\mathbf{L H}_{t o p} \downarrow X$.

\footnotetext{
${ }^{2}$ Aliás, embora tenhamos apresentado o funtor $C$ como um feixe de conjuntos, ele é também um feixe de $\mathbb{R}$-álgebras (ou $\mathbb{R}$-módulos), pois cada $C(U, X)$ é uma $\mathbb{R}$-álgebra.

${ }^{3}$ Em inglês, $F_{x}$ é chamado de "stalk", cuja tradução literal seria "caule".
} 
Como esses resultados sugerem, esses funtores definem a relação entre as categorias de feixes geométricos e feixes funtoriais, o que é expresso pelos seguintes dois teoremas:

Teorema 2.1.14. Seja $(X, \mathcal{O}(X))$ um espaço topológico. Então, os funtores

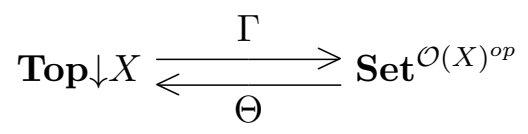

definem uma adjunção $\Theta \dashv \Gamma$.

Teorema 2.1.15. Seja $(X, \mathcal{O}(X))$ um espaço topológico. Os funtores $\Gamma$ e $\Theta$ se restringem a uma equivalência de categorias $\mathbf{L H}_{t o p} \downarrow X \simeq \mathbf{S h}(X)$.

Para a demonstração detalhada desses resultados, pode-se consultar as seções 2.3 e 2.4 de [Ten75].

\section{$2.2 \quad$ Feixes sobre locales}

Na definição usual de um espaço topológico, temos um par $(X, \mathcal{O}(X))$, em que $X$ é um conjunto, o "conjunto dos pontos", e $\mathcal{O}(X)$ é um conjunto de subconjuntos de $X$, os chamados abertos de $X$; que satisfaz: $X, \emptyset \in \mathcal{O}(X)$, união arbitrária de abertos é aberto e intersecção finita de abertos é aberto. O importante para as construções de feixes sobre espaços topológicos, no entanto, são os abertos, e não o conjunto de pontos em si. Assim, os locales são definidos para reter parte da estrutura do conjunto de abertos.

Definição 2.2.1. Seja $(L, \leq)$ um reticulado completo ${ }^{4}$. $L$ é dito um locale se, para todo $a \in L$ e toda família $\left\{b_{i} \in L \mid i \in I\right\}$, vale

$$
a \wedge\left(\bigvee_{i \in I} b_{i}\right)=\bigvee_{i \in I}\left(a \wedge b_{i}\right)
$$

Exemplo 2.2.2. Dado um espaço topológico $(X, \mathcal{O}(X))$, é evidente que $(\mathcal{O}(X), \subseteq)$ é um locale; as operações de supremo arbitrário e ínfimo finito coincidem com as operações de união e interseç̧ão (e resultam em abertos por ser um espaço topológico), e o ínfimo arbitrário é o interior da interseç̧ão arbitrária (pois, ao contrário do caso finito, interseç̧ão infinita de abertos pode

\footnotetext{
${ }^{4}$ Poderíamos definir usando apenas um reticulado com supremos arbitrários e ínfimos finitos em vez de um reticulado completo, o que talvez deixasse mais clara a relação com os abertos de um espaço topológico, e seria mais compatível com a definição de morfismo de locales a seguir. Porém, pelo teorema do funtor adjunto (1.7.7), um reticulado assim também possui ínfimos arbitrários.
} 
não ser aberto). Dessa forma, a distributividade exigida na definição decorre da distributividade das operações de conjuntos.

Proposição 2.2.3. Seja $(L, \leq)$ um reticulado. Então, são equivalentes:

1. $(L, \leq)$ é um locale;

2. $(L, \leq)$ é uma álgebra de Heyting completa.

Demonstração. $(1 \Rightarrow 2)$ Para todos $a, b \in L$, defina $a \rightarrow b=\bigvee\{c \in L \mid c \wedge a \leq b\}$. Assim, seja $c \in L$. É imediato que se $c \wedge a \leq b$, então $c \leq(a \rightarrow b)$. Por outro lado, se $c \leq(a \rightarrow b)$, então:

$$
c \wedge a \leq(a \rightarrow b) \wedge a=(\bigvee\{d \in L \mid d \wedge a \leq b\}) \wedge a=\bigvee\{d \wedge a \mid d \in L \text { e } d \wedge a \leq b\} \leq b
$$

Logo $c \wedge a \leq b$ se, e somente se, $c \leq(a \rightarrow b)$, como desejado.

(2 $\Rightarrow 1$ ) Sejam $a \in L$ e $\left\{b_{i} \in L \mid i \in I\right\}$ uma família de elementos de $L$. Primeiramente, $\bigvee_{i \in I} b_{i} \geq b_{i}$ para todo $i \in I$, logo $a \wedge \bigvee_{i \in I} b_{i} \geq a \wedge b_{i}$, para todo $i \in I$, donde obtemos $a \wedge \bigvee_{i \in I} b_{i} \geq$ $\bigvee_{i \in I}^{i \in I}\left(a \wedge b_{i}\right)$. Reversamente, chamando $S=\bigvee_{i \in I}\left(a \wedge b_{i}\right)$, temos, para todo $i \in I, a \wedge b_{i} \leq S$, o que é equivalente a $b_{i} \leq a \rightarrow S$. Portanto, $\bigvee_{i \in I} b_{i} \leq a \rightarrow S$, e $a \wedge \bigvee_{i \in I} b_{i} \leq S$. Assim, $a \wedge \bigvee_{i \in I} b_{i}=\bigvee_{i \in I}\left(a \wedge b_{i}\right)$, e concluímos que $(L, \leq)$ é um locale.

Definição 2.2.4. Sejam $(L, \leq),\left(L^{\prime}, \leq^{\prime}\right)$ locales. Um morfismo de locales $f: L \rightarrow L^{\prime}$ é um funtor que possui um adjunto à esquerda $f^{*}: L^{\prime} \rightarrow L$ que preserva ínfimos finitos. Assim, definimos a categoria Loc, que tem locales como objetos e morfismos de locales como flechas.

Note que, como isos são a igualdade em categorias de ordens parciais, $f^{*}$ é único.

Proposição 2.2.5. Sejam $(L, \leq),\left(L^{\prime}, \leq^{\prime}\right)$ locales. Então, existe uma bijeção entre os morfismos de locales $f: L \rightarrow L^{\prime}$ e as funções $g: L^{\prime} \rightarrow L$ que preservam supremos arbitrários e ínfimos finitos. Isto é, para todos $a^{\prime}, b^{\prime} \in L^{\prime}$ e toda família $\left\{a_{i}^{\prime} \in L^{\prime} \mid i \in I\right\}, g$ satisfaz:

$$
\begin{gathered}
g\left(\bigvee_{i \in I}^{\prime} a_{i}^{\prime}\right)=\bigvee_{i \in I} g\left(a_{i}^{\prime}\right) \\
g\left(a^{\prime} \wedge^{\prime} b^{\prime}\right)=g\left(a^{\prime}\right) \wedge g\left(b^{\prime}\right) \\
g\left(1^{\prime}\right)=1
\end{gathered}
$$

Demonstração. Dado um morfismo de locales $f: L \rightarrow L^{\prime}$, por hipótese $f$ determina unicamente 
uma função $f^{*}$ que preserva ínfimos finitos (inclusive o ínfimo vazio, que é a unidade do locale), e a adjunção $f^{*} \dashv f$ garante que $f^{*}$ preserve colimites (logo supremos) arbitrários.

Agora, seja $g: L^{\prime} \rightarrow L$ como no enunciado. Note que $g: L^{\prime} \rightarrow L$ é um funtor e satisfaz a as condições do resultado dual ao teorema do funtor adjunto 1.7.7. De fato, por definição $L^{\prime}$ é uma categoria pequena e cocompleta, e $g$ preserva colimites (supremos). Para a condição conjunto solução (dual), dado $a \in L$, basta tomar a família de todas as flechas $\left\{g\left(a^{\prime}\right) \rightarrow a\right.$ em $L^{o p} \mid a^{\prime} \in$ $\left.L^{\prime}\right\}$, pois uma flecha $g\left(a^{\prime}\right) \rightarrow a$ em $L^{o p}$ indica simplesmente a desigualdade $a \leq g\left(a^{\prime}\right)$. Logo, $g$ possui um (único) adjunto à direita $g_{*}$, e $g_{*}: L \rightarrow L^{\prime}$ é um morfismo de locales.

Por conta dessa proposição, alguns autores definem a categoria Loc de outra maneira: primeiramente, considera-se Frm a categoria dos frames, que tem como objetos locales e flechas as funções que preservam supremos arbitrários e ínfimos finitos. Com isso, a categoria dos locales é definida como Loc $:=$ Frm $^{o p}$.

Exemplo 2.2.6. Considere uma função contínua $f: X \rightarrow X^{\prime}$, para $(X, \mathcal{O}(X))$ e $\left(X^{\prime}, \mathcal{O}\left(X^{\prime}\right)\right)$ espaços topológicos. Essa função induz uma função imagem inversa $f^{-1}: \mathcal{O}\left(X^{\prime}\right) \rightarrow \mathcal{O}(X)$, que leva cada aberto $U^{\prime} \in \mathcal{O}\left(X^{\prime}\right)$ a um aberto $f^{-1}\left(U^{\prime}\right) \in \mathcal{O}(X)$. Tal função $f^{-1}$ preserva supremos arbitrários e ínfimos finitos. Assim, podemos definir um morfismo de locales $\varphi: \mathcal{O}(X) \rightarrow \mathcal{O}\left(X^{\prime}\right)$ correspondente à função $f$ fazendo $\varphi(U)=\bigcup\left\{V \in \mathcal{O}\left(X^{\prime}\right) \mid f^{-1}(V) \subseteq U\right\}$, e o adjunto à esquerda é $\varphi^{*}=f^{-1}$.

Definição 2.2.7. Seja $(L, \leq)$ um locale e $a \in L$. Definimos o segmento superior e o segmento inferior de $a$, respectivamente, como: $\uparrow a:=\{b \in L \mid a \leq b\}$ e $\downarrow a:=\{b \in L \mid b \leq a\}$. É fácil verificar que ambos são sublocales de $L$ (não preservam a unidade de $L$, mas preservam ínfimos binários e supremos arbitrários).

As definições de seções, e de prefeixe e feixe funtorial podem ser ser reescritas quase sem modificações para locales, dado que usam apenas abertos e coberturas de abertos. Por outro lado, usamos pontos para definir feixes geométricos, porém não seria necessário: o mais importante para definir eram as vizinhanças abertas, e não os pontos. Façamos com detalhes abaixo.

Definição 2.2.8. Sejam $(L, \leq),\left(L^{\prime}, \leq^{\prime}\right)$ locales. Um feixe geométrico sobre $L^{\prime}$ é um homeomorfismo local $f: L \rightarrow L^{\prime}$ de locales, isto é, existem famílias $\left\{U_{j} \in L \mid j \in J\right\}$ com $\bigvee_{j \in J} U_{j}=1$ e $\left\{U_{j}^{\prime} \in L^{\prime} \mid j \in J\right\}$ tais que a restrição $f_{\mid \downarrow U_{j}}: \downarrow U_{j} \rightarrow \downarrow U_{j}^{\prime}$ é um iso, para todo $j \in J$. Denotaremos por $\mathbf{L H}_{l o c}$ a categoria dos locales e homeomorfismos locais de locales. A categoria slice $\mathbf{L H}_{l o c} \downarrow L^{\prime}$ é chamada de categoria dos feixes geométricos sobre $L^{\prime}$. 
Outra forma de definir seria usando um recobrimento de $L$ por uma família de sublocales $\left\{L_{j} \subseteq L \mid j \in J\right\}$ (no sentido de para todo $U \in L$ existe uma família $\left\{U_{j} \in L \mid j \in J\right\}$ tal que $\bigvee_{j \in J} U_{j}=U$ e $U_{j} \in L_{j}$, para todo $\left.j \in J\right)$. A definição de homeomorfismo local de locales $f$, então, seria que existem um recobrimento $\left\{L_{j} \subseteq L \mid j \in J\right\}$ de $L$ e uma família $\left\{L_{j}^{\prime} \subseteq L^{\prime} \mid j \in J\right\}$ de sublocales de $L^{\prime}$ tais que $f \circ i_{L_{j}} \cong i_{L_{j}^{\prime}}$, onde $i_{L_{j}}: L_{j} \hookrightarrow L$ e $i_{L_{J}^{\prime}}: L_{j}^{\prime} \hookrightarrow L^{\prime}$ denotam as inclusões, para cada $j \in J$.

Exemplo 2.2.9. Verifiquemos que homeomorfismos locais entre espaços topológicos dão origem a homeomorfismos locais de locales entre as respectivas topologias. Sejam $(X, \mathcal{O}(X))$ e $(Y, \mathcal{O}(Y))$ espaços topológicos e $f: X \rightarrow Y$ um homeomorfismo local. Para cada $x \in X$, considere $U_{x} \in \mathcal{O}(X)$ o aberto com $x \in U_{x}$ tal que existe $V_{x} \in \mathcal{O}(Y)$ com $f(x) \in V_{x}$ satisfazendo $f_{\mid U}: U_{x} \rightarrow V_{x}$ ser um homeomorfismo, como na definição. Dessa forma, obtemos as famílias de abertos $\left\{U_{x} \in \mathcal{O}(X) \mid x \in X\right\}$, com $\bigcup_{x \in X} U_{x}=X$, e $\left\{V_{x} \in \mathcal{O}(Y) \mid x \in X\right\}$. Defina $\varphi: \mathcal{O}(X) \rightarrow \mathcal{O}(Y)$ e $\varphi^{*}: \mathcal{O}(Y) \rightarrow \mathcal{O}(X)$ como no exemplo 2.2.6 e denote as restrições por $\varphi_{x}:=\varphi_{\mid \downarrow U_{x}}: \downarrow U_{x} \rightarrow \downarrow V_{x}$ e $\varphi_{x}^{*}:=\varphi_{\mid \downarrow V_{x}}^{*}: \downarrow V_{x} \rightarrow \downarrow U_{x}$. Mostremos que, para todo $x \in X$, $\varphi_{x}^{-1}=\varphi_{x}^{*}$, isto é, que $\varphi_{x}$ é um iso.

Primeiramente, seja $U^{\prime} \in \downarrow U_{x}$. Então:

$$
\varphi_{x}^{*}\left(\varphi_{x}\left(U^{\prime}\right)\right)=\varphi_{x}^{*}\left(\bigcup\left\{v \in \downarrow V_{x} \mid f^{-1}(v) \subseteq U^{\prime}\right\}\right)=f^{-1}\left(\bigcup\left\{v \in \downarrow V_{x} \mid f^{-1}(v) \subseteq U^{\prime}\right\}\right)
$$

Com isso, para todo $a \in X$, temos:

$$
\begin{aligned}
a \in f^{-1}\left(\bigcup\left\{v \in \downarrow V_{x} \mid f^{-1}(v) \subseteq U^{\prime}\right\}\right) & \Leftrightarrow f(a) \in \bigcup\left\{v \in \downarrow V_{x} \mid f^{-1}(v) \subseteq U^{\prime}\right\} \\
& \Leftrightarrow \exists v \in \downarrow V_{x}\left(f(a) \in v \text { e } f^{-1}(v) \subseteq U^{\prime}\right) \\
& \Leftrightarrow a \in U^{\prime}
\end{aligned}
$$

(usando que $f_{\mid U_{x}}$ é homeomorfismo na última implicação $\Leftarrow$ ). Ou seja, $\varphi_{x}^{*} \circ \varphi_{x}=i d_{\downarrow U_{x}}$.

Agora, seja $V^{\prime} \in \downarrow V_{x}$. Então,

$$
\varphi_{x}\left(\varphi_{x}^{*}\left(V^{\prime}\right)\right)=\varphi_{x}\left(f^{-1}\left(V^{\prime}\right)\right)=\bigcup\left\{v \in \downarrow V_{x} \mid f^{-1}(v) \subseteq f^{-1}\left(V^{\prime}\right)\right\}
$$

Ademais, para todo $b \in V_{x}$, como $f_{\mid U_{x}}$ é homeomorfismo, existe $a \in U_{x}$ tal que $f(a)=b$. Então, para todo $v \in \downarrow V_{x}$ tal que $f^{-1}(v) \subseteq f^{-1}\left(V^{\prime}\right)$, temos

$$
b \in v \Leftrightarrow f(a) \in v \Rightarrow f(a) \in V^{\prime} \Leftrightarrow b \in V^{\prime}
$$


isto é, $v \subseteq V^{\prime}$. E como é claro que $f^{-1}\left(V^{\prime}\right) \subseteq f^{-1}\left(V^{\prime}\right)$, vale:

$$
\bigcup\left\{v \in \downarrow V_{x} \mid f^{-1}(v) \subseteq f^{-1}\left(V^{\prime}\right)\right\}=V^{\prime}
$$

de forma que $\varphi_{x} \circ \varphi_{x}^{*}=i d_{\downarrow} V_{x}$.

As fibras $F_{x}$ e o espaço topológico $E^{(F)}$, cujas definições dependiam de pontos, foram usados para mostrar a equivalência entre ambas as noções de feixes no caso topológico, então não é possível proceder da mesma forma com locales. Porém, existe uma noção que ajuda a transpor a equivalência no caso dos locales:

Definição 2.2.10. Sejam $(L, \leq)$ um locale e $F: L^{o p} \rightarrow$ Set um prefeixe. Um subprefeixe ${ }^{5}$ $G: L^{o p} \rightarrow$ Set de $F$ é dito um subprefeixe fechado se para todo $U \in L$, para toda família $\left\{U_{j} \in L \mid j \in J\right\}$ com $\bigvee_{j \in J} U_{j}=U$ e todo $s \in F_{0}(U)$, temos: se para todo $j \in J,\left.s\right|_{U_{j}} ^{U} \in G_{0}\left(U_{j}\right)$, então $s \in G_{0}(U)$. O conjunto dos subprefeixes de $F$ constitui um locale.

A proposição a seguir mostra a relação dos subprefeixes fechados com o espaço $E^{(F)}$, de forma que pode-se usar os subprefeixes fechados em vez das fibras para mostrar a equivalência no caso dos locales.

Proposição 2.2.11. Sejam $(X, \mathcal{O}(X))$ um espaço topológico e $F: \mathcal{O}(X)^{o p} \rightarrow$ Set um prefeixe. Então, o locale dos abertos de $E^{(F)}$ é isomorfo ao locale dos subprefeixes fechados de $F$.

Uma importante motivação para considerar feixes sobre locales é que, ao contrário do que ocorre com espaços topológicos, a passagem da categoria dos locales para a categoria dos feixes sobre locales dá origem a um funtor pleno e fiel. Façamos aqui uma breve apresentação desses resultados, tendo como base as seções 1.8 e 1.9 de [Bor08c], e as seções IX.2 e IX.3 de [LM94].

Comecemos definindo pontos de um locale, para podermos introduzir o par de funtores adjuntos entre espaços topológicos e locales. Há três formas equivalentes de definir pontos:

Definição 2.2.12. Seja $(L, \leq)$ um locale. Um ponto de $L$ é um morfismo $p:\{0,1\} \rightarrow L$ (é fácil verificar que $\{0,1\}$ é o locale terminal). O conjunto de pontos de um locale será denotado por $p t(L):=\operatorname{Loc}(\{0,1\}, L)$.

Definição 2.2.13. Sejam $(L \leq)$ um locale e $a \in L$. Dizemos que $a$ é primo se $a \neq 1$ e, para todos $b, c \in L$, vale: $b \wedge c \leq a$ implica em $b \leq a$ ou $c \leq a$.

\footnotetext{
${ }^{5}$ Ou seja, um subfuntor, como definido em 1.6.3
} 
Definição 2.2.14. Sejam $(L, \leq)$ um locale e $A \subseteq L$. Dizemos que $A$ é um filtro (próprio) se:

1. $1 \in A$;

2. $0 \notin A$;

3. para todos $a \in A$ e $b \in L$, se $a \leq b$ então $b \in A$;

4. para todos $a, a^{\prime} \in A, a \wedge a^{\prime} \in A$.

A é dito um filtro primo se, além disso, satisfaz:

5. para todos $a, a^{\prime} \in A$, se $a \vee a^{\prime} \in A$, então $a \in A$ ou $a^{\prime} \in A$.

A é dito um filtro completamente primo se, além disso, satisfaz:

6. para toda família $\left\{a_{i} \in L \mid i \in I\right\}$, se $\bigvee_{i \in I} a_{i} \in A$, então existe $i \in I$ tal que $a_{i} \in A$.

Proposição 2.2.15. Seja $(L, \leq)$ um locale. Então, existe uma bijeção entre:

1. os pontos de L;

2. os elementos primos de $L$;

3. os filtros completamente primos de L.

Não façamos a demonstração completa aqui, mas indiquemos as bijeções. Dado um ponto $p$ de $L$, podemos definir um elemento primo $a$ de $L$ fazendo:

$$
a=\bigvee\left\{b \in L \mid p^{*}(b)=0\right\}
$$

Reversamente, podemos construir um ponto $p$ a partir de um elemento primo $a$ definindo $p^{*}$ : $L \rightarrow\{0,1\}$ como:

$$
p^{*}(b)= \begin{cases}0 & , \text { se } b \leq a \\ 1 & , \text { caso contrário }\end{cases}
$$

(ou seja, $p(0)=a$ e $p(1)=1$ ). Agora, seja $a$ um elemento primo de $L$, e considere $A \subseteq L$ dado por $A=\{b \in L \mid b \not \leq a\}$, que será um filtro completamente primo. Finalmente, dado um filtro completamente primo $A$, podemos definir um elemento primo $a$ de $L$ como:

$$
a=\bigvee\{b \in L \mid b \notin A\}
$$


Exemplo 2.2.16. Sejam $(X, \mathcal{O}(X))$ um espaço topológico e $x \in X$. Podemos definir um ponto $p_{x}:\{0,1\} \rightarrow \mathcal{O}(X)$ correspondente a $x$ tomando

$$
p_{x}(0)=\bigcup\{U \in \mathcal{O}(X) \mid x \notin U\} \quad \text { e } \quad p_{x}(1)=X
$$

com o adjunto à esquerda satisfazendo, para todo $U \in \mathcal{O}(X)$,

$$
p_{x}^{*}(U)=1 \quad \text { se, e somente se, } \quad x \in U
$$

Observe que $p_{x}$ é exatamente o morfismo de locales correspondente a uma função contínua $f_{x}:\{*\} \rightarrow X$ tal que $f_{x}(*)=x$ (com $\{*\}$ um espaço topológico com um elemento, de forma que sua topologia é o locale terminal). Note, também, que $\bigcup\{U \in \mathcal{O}(X) \mid x \notin U\}$ é um elemento primo de $\mathcal{O}(X)$, e é o complemento de $\overline{\{x\}}$.

Esse exemplo sugere uma forma de definir uma topologia no conjunto de pontos de um locale:

Lema 2.2.17. Seja $(L, \leq)$ um locale. Para cada $a \in L$, defina

$$
\mathcal{O}_{a}:=\left\{p \in p t(L) \mid p^{*}(a)=1\right\}
$$

Então, para todos $a, b \in L$ e para toda família $\left\{a_{i} \in L \mid i \in I\right\}$, vale:

1. $\mathcal{O}_{0}=\emptyset e \mathcal{O}_{1}=p t(L)$;

2. $\mathcal{O}_{i \in I} a_{i}=\bigcup_{i \in I} \mathcal{O}_{a_{i}}$

3. $\mathcal{O}_{a \wedge b}=\mathcal{O}_{a} \cap \mathcal{O}_{b}$.

Ou seja, $\left\{\mathcal{O}_{a} \subseteq p t(L) \mid a \in L\right\}$ define uma topologia em $p t(L)$.

Teorema 2.2.18. Seja $\mathcal{O}:$ Top $\rightarrow$ Loc o funtor que leva cada espaço topológico $(X, \mathcal{O}(X))$ a seu locale de abertos $\mathcal{O}(X)$, e cada função continua a seu morfismo de locales correspondente (como no exemplo 2.2.6). Seja pt: Loc $\rightarrow$ Top o funtor que leva cada locale $L$ a seu conjunto de pontos pt $(L)$, munido da topologia $\left\{\mathcal{O}_{a} \mid a \in L\right\}$, e cada morfismo de locales $f: L \rightarrow L^{\prime} a$ uma função contínua $p t_{1}(f): p t(L) \rightarrow p t\left(L^{\prime}\right)$ com $p t_{1}(f)(p)=f \circ p$. Esses funtores definem uma $\operatorname{adjunção~} \mathcal{O} \dashv p t$. 
A unidade $\eta: i d_{\text {Top }} \Rightarrow p t \circ \mathcal{O}$ da adjunção é dada por, para cada $X \in \mathbf{T o p}_{0}$,

$$
\eta_{X}: X \rightarrow p t(\mathcal{O}(X)), \operatorname{com} \eta_{X}(x)=p_{x}, \text { para todo } x \in X
$$

e a counidade $\theta: \mathcal{O} \circ p t \Rightarrow i d_{\text {Loc }}$ da adjunção é dada por, para cada $L \in \mathbf{L o c}_{0}$,

$$
\theta_{L}: \mathcal{O}(p t(L)) \rightarrow L, \operatorname{com} \theta_{L}^{*}(a)=\mathcal{O}_{a}, \text { para todo } a \in L
$$

Observe, no entanto, que $\mathcal{O}$ não é pleno nem fiel. Por exemplo considere os espaços topológicos $X=\{*\}$ e $Y=\{0,1\}$ munidos das topologias $\mathcal{O}(X)=\{\emptyset,\{*\}\}$ e $\mathcal{O}(Y)=\{\emptyset,\{0,1\}\}$. Sejam $f, g: X \rightarrow Y$ dadas por $f(*)=0$ e $g(*)=1$, ambas contínuas (pois $Y$ está munido da topologia trivial). Agora, as funções imagem inversa $f^{-1}, g^{-1}: \mathcal{O}(Y) \rightarrow \mathcal{O}(X)$, que definem morfismos entre os locales de abertos, são ambas dadas por $f^{-1}(\emptyset)=\emptyset=g^{-1}(\emptyset)$ e $f^{-1}(\{0,1\})=\{*\}=g^{-1}(\{0,1\})$. Ou seja, $\mathcal{O}_{1}(f)=\mathcal{O}_{1}(g)$, mas $f \neq g$, logo $\mathcal{O}$ não é fiel.

Ademais, considere $\varphi^{*}: \mathcal{O}(Y) \rightarrow \mathcal{O}(X)$ dada por $\varphi^{*}(\emptyset)=\varphi^{*}(\{0,1\})=\emptyset$. Claramente isso define um morfismo de locales $\varphi: \mathcal{O}(X) \rightarrow \mathcal{O}(Y)$, mas como vimos toda função $X \rightarrow Y$ em $\mathbf{T o p}_{1}$ é levada ao mesmo morfismo em $\mathbf{L o c}_{1}$ (o morfismo com adjunto à esquerda $f^{-1}$ como acima), então não existe $h: X \rightarrow Y$ tal que $\varphi=\mathcal{O}_{1}(h)$, donde concluímos que $\mathcal{O}$ não é pleno.

Não obstante, a adjunção restringe-se a uma equivalência de categorias para locales e espaços topológicos específicos.

Definição 2.2.19. Seja $(L, \leq)$ um locale. Dizemos que $L$ tem suficientes pontos se, para todos $a, b \in L, \mathcal{O}_{a}=\mathcal{O}_{b}$ implica $a=b$. Denotamos a categoria dos locales com suficientes pontos e seus morfismos por spLoc.

Essa definição remonta ao fato de que, num espaço topológico, conjuntos abertos são iguais se possuem os mesmos pontos. Na proposição abaixo, enunciamos formas equivalentes da definição.

Proposição 2.2.20. Sejam $(L, \leq)$ um locale e $a, b \in L$. Então, são equivalentes:

\section{L tem suficientes pontos;}

2. $\theta_{L}: \mathcal{O}(p t(L)) \rightarrow L$ é um isomorfismo;

3. se $a \not \leq b$, então existe $p \in p t(L)$ tal que $p^{*}(a)=1$ e $p^{*}(b)=0$;

4. se $a \not \leq b$, então existe $c \in L$ primo tal que $a \not \leq c$ e $b \leq c$; 
5. se $a \not \leq b$, então existe $A \subseteq L$ um filtro completamente primo tal que $a \in A$ e $b \notin A$.

Definição 2.2.21. Seja $(X, \mathcal{O}(X))$ um espaço topológico. Um conjunto fechado $C \subseteq X$ é dito um fechado irredutivel se $C \neq \emptyset$ e satisfaz, para todos fechados $C_{1}, C_{2} \subseteq X$,

$$
\text { se } C \subseteq C_{1} \cup C_{2} \text {, então } C \subseteq C_{1} \text { ou } C \subseteq C_{2}
$$

Observe que essa noção é dual à noção de ser primo num locale. Assim, um fechado é irredutível se seu complemento é um elemento primo no locale dos abertos.

Ademais, note que, para todo $x \in X, \overline{\{x\}}$ é um fechado irredutível.

Definição 2.2.22. Seja $(X, \mathcal{O}(X))$ um espaço topológico. Dizemos que $X$ é sóbrio se, para todo fechado irredutível $C \subseteq X$, existe um único $x \in X$ tal que $C=\overline{\{x\}}$. Denotamos a categoria dos espaços sóbrios e funções contínuas entre eles por sbTop.

Todo espaço Hausdorff $\left(T_{2}\right)$ é sóbrio, e todo espaço sóbrio é $T_{0}$, porém as recíprocas não são verdadeiras.

Novamente, temos formas equivalentes de definir espaços sóbrios:

Proposição 2.2.23. Seja $(X, \mathcal{O}(X))$ um espaço topológico. Então, são equivalentes:

1. X é sóbrio;

2. $\eta_{X}: X \rightarrow p t(\mathcal{O}(X))$ é um homeomorfismo;

3. $\eta_{X}: X \rightarrow p t(\mathcal{O}(X))$ é uma bijeção;

Com isso, podemos enunciar os resultados que mostram a relação entre espaços sóbrios e locales com suficientes pontos.

Proposição 2.2.24. Seja $(L, \leq)$ um locale. Então, pt $(L)$ é um espaço sóbrio.

Proposição 2.2.25. Seja $(X, \mathcal{O}(X))$ um espaço topológico. Então, $\mathcal{O}(X)$ é um locale com suficientes pontos.

Teorema 2.2.26. A adjunção $\mathcal{O} \dashv p t$ restringe-se a uma equivalência de categorias 
Enfim, consideremos a passagem de um locale para o conjunto dos feixes sobre tal locale.

Definição 2.2.27. Considere $\mathcal{C}$ a categoria das categorias localmente pequenas com flechas os funtores que possuem um adjunto à esquerda que preserva limites finitos (esses funtores serão chamados mais adiante de morfismos geométricos). Assim, definimos o funtor $\mathbf{S h}:$ Loc $\rightarrow \mathcal{C}$ como o funtor que leva cada locale $L$ à categoria $\mathbf{S h}(L)$ dos feixes sobre $L$, e cada morfismo de locales $f: L \rightarrow L^{\prime}$ ao funtor $\mathbf{S h}_{1}(f): \mathbf{S h}(L) \rightarrow \mathbf{S h}\left(L^{\prime}\right)$ tal que, para todo $F \in \mathbf{S h}(L)$, $\mathbf{S h}_{1}(f)(F)=F \circ f^{*}$.

Teorema 2.2.28. O funtor $\mathbf{S h}:$ Loc $\rightarrow \mathcal{C}$ é pleno e fiel.

A demonstração desse teorema será postergada até o último capítulo, onde estaremos interessados em topoi equivalentes à categoria de feixes sobre um locale.

Como consequência desse teorema, usando que $\mathcal{O}$ não é nem pleno nem fiel, o funtor $\mathbf{S h} \circ \mathcal{O}$ : Top $\rightarrow \mathcal{C}$ também não é nem pleno nem fiel.

Para finalizar essa seção, introduzimos a noção de uma cobertura hereditária, que será conveniente para fazer analogias de construções em locales com construções mais gerais na seção seguinte.

Definição 2.2.29. Sejam $(P, \leq)$ um conjunto parcialmente ordenado e $a \in P$. Um subconjunto $S \subseteq P$ é dito hereditário (ou fechado para baixo) se para todo $p \in P$ e para todo $u \in S$, se $p \leq u$ então $p \in C$. Um subconjunto $C \subseteq P$ é dito uma cobertura hereditária de $a$ se $C$ for hereditário e $\bigvee C=a$.

Observe que $\downarrow a$ é sempre uma cobertura hereditária de $a$.

Lema 2.2.30. Sejam $(P, \leq)$ um conjunto parcialmente ordenado e a $\in P$. Então, existe uma bijeção entre os subconjuntos hereditários de $\downarrow$ a e os subfuntores de $P(-, a)$.

Isso é fácil se pensarmos na estrutura de $P$ como uma categoria e na definição de subfuntor. O conjunto $P(b, a)$ será unitário se $b \leq a$ e vazio caso contrário, assim um subfuntor $G$ de $P(-, a)$ consiste em escolher $b \leq a$ tais que $G_{0}(b)=\{*\}$ e, para todo $c \leq b, G_{0}(b)=\{*\}$ implica $G_{0}(c)=\{*\}$ (pela funtorialidade de $G$ ), como na definição de subconjunto hereditário. Naturalmente, dessa forma $\downarrow a$ corresponde ao próprio funtor $P(-, a)$.

Lema 2.2.31. Sejam $(L, \leq)$ um locale, $F: L^{o p} \rightarrow$ Set um prefeixe e a $\in$ L. Para todo subconjunto hereditário $S \subseteq \downarrow$ a, com subfuntor $\tilde{S}$ de $L(-, a)$ correspondente (pela proposição anterior), 
existe uma bijeção entre as famílias F-compativeis $\left\{s_{b} \in F_{0}(b) \mid b \in S\right\}$ e as transformações naturais $\eta: \tilde{S} \Rightarrow F$.

De fato, uma transformação natural $\eta: \tilde{S} \Rightarrow F$ consiste em escolher, para todo $b \in L$, um elemento $\eta_{b}(*) \in F_{0}(b)$ tal que $\tilde{S}_{0}(b)=\{*\}$ (logo $b \in S$ ), e a naturalidade corresponde à compatibilidade da família $\left\{\eta_{b}(*) \in F_{0}(b) \mid b \in S\right\}$.

Com esses lemas, obtemos uma outra forma de definir feixes:

Proposição 2.2.32. Sejam $(L, \leq)$ um locale e $F$ um prefeixe sobre L. Então, são equivalentes:

1. F é um feixe sobre $L$;

2. para todo $a \in L$ e todo subfuntor $G$ de $L(-, a)$, toda transformação natural $\eta: G \Rightarrow F$ pode ser estendida unicamente a uma transformação natural $\tilde{\eta}: L(-, a) \Rightarrow F$.

Pelas proposições anteriores, para todo $b \leq a$, as escolhas únicas das $\tilde{\eta}_{b}(*) \in F_{0}(b)$ (para $\tilde{\eta}_{b}$ as componentes da transformação natural) devem corresponder exatamente à colagem única $s \in F_{0}(a)$ tal que $\left.s\right|_{b} ^{a}=s_{b} \in F_{0}(b)$ obtida da condição de feixe. Portanto, as condições (1) e (2) são equivalentes para coberturas hereditárias.

Para uma cobertura $\left\{a_{i} \in L \mid i \in I\right\}$ de a qualquer, dada uma família F-compaível $\left\{s_{i} \in\right.$ $\left.F_{0}\left(a_{i}\right) \mid i \in I\right\}$, podemos definir uma cobertura hereditária de $a$ fazendo:

$$
C=\left\{b \in L \mid \text { existe } i \in I \text { tal que } v \leq a_{i}\right\}
$$

e, por ser compatível, a família $\left\{s_{i} \in F_{0}\left(a_{i}\right) \mid i \in I\right\}$ pode ser estendida a uma família F-compaível $\left\{s_{b} \in F_{0}(b) \mid b \in C\right\}$.

\subsection{Feixes sobre sítios e topos de Grothendieck}

Vimos, até agora, como construir feixes usando espaços topológicos e locales. Porém, em outros contextos matemáticos, inclusive em geometria algébrica (onde surgiu a primeira noção de topos), uma certa "intuição topológica" estava presente, mas não dava origem a um locale. Essa intuição topológica referia-se a algo que se assemelhava a coberturas, e é essa noção que será formalizada a seguir. 
Definição 2.3.1. Seja $\mathcal{C}$ uma categoria pequena com pullbacks binários. Uma pretopologia de Grothendieck (ou base de Grothendieck) em $\mathcal{C}$ é uma função $\operatorname{cov}: \mathcal{C}_{0} \rightarrow \mathcal{P}\left(\mathcal{P}\left(\mathcal{C}_{1}\right)\right.$ ), $\operatorname{com} \operatorname{cov}(c) \in$ $\mathcal{P}\left(\mathcal{P}\left(\bigcup_{a \in \mathcal{C}_{0}} \mathcal{C}(a, c)\right)\right)$ para todo $\left.c \in \mathcal{C}_{0}\right)$ (de forma que $E \in \operatorname{cov}(c)$ implica $E \subseteq \bigcup_{a \in \mathcal{C}_{0}} \mathcal{C}(a, c)$ ), satisfazendo:

1. iso: se $f: a \rightarrow c$ é iso, então $\{f\} \in \operatorname{cov}(c)$;

2. fechado por composição: se $\left\{f_{i}: b_{i} \rightarrow c \mid i \in I\right\} \in \operatorname{cov}(c)$ e, para todo $i \in I,\left\{g_{i j}: a_{i j} \rightarrow\right.$ $\left.b_{i} \mid j \in J\right\} \in \operatorname{cov}\left(b_{i}\right)$, então $\left\{f_{i} \circ g_{i j}: a_{i j} \rightarrow c \mid i \in I, j \in J\right\} \in \operatorname{cov}(c)$;

3. estabilidade por pullbacks: para toda família $\left\{f_{i}: b_{i} \rightarrow c \mid i \in I\right\} \in \operatorname{cov}(c)$ e para toda flecha $g: a \rightarrow c$, temos $\left\{f_{i}^{\prime}: p_{i} \rightarrow a \mid i \in I\right\} \in \operatorname{cov}(a)$ (onde $\left(p_{i}, f_{i}^{\prime}, g^{\prime}\right)$ é o pullback de $f_{i}$ e $g$, para cada $i \in I)$;

4. monotonicidade: para toda família $\left\{f_{i}: b_{i} \rightarrow c \mid i \in I\right\} \subseteq \bigcup_{a \in \mathcal{C}_{0}} \mathcal{C}(a, c)$, se existir uma família $\left\{g_{j}: a_{j} \rightarrow c \mid j \in J\right\} \in \operatorname{cov}(c)$ tal que $\forall j \in J, \exists i \in I, \exists h_{i j}: a_{j} \rightarrow b_{i}\left(f_{i} \circ h_{i j}=g_{j}\right)$, então $\left\{f_{i}: b_{i} \rightarrow c \mid i \in I\right\} \in \operatorname{cov}(c)$.

Exemplo 2.3.2. A pretopologia de Grothendieck associada a um locale $(L, \leq)$ é dada por $\operatorname{cov}(c)=\left\{f: a_{i} \rightarrow c \mid i \in I, \bigvee_{i \in I} a_{i}=c\right\}$. Lembrando que $f: a_{i} \rightarrow c$ significa $a_{i} \leq c$, e o pullback em $L$ é dado pelo ínfimo $\wedge$.

Essa noção de cobertura, porém, não satisfaz certas propriedades importantes de "saturação" ou "fechamento" que estão presentes em espaços topológicos e locales, embora não sejam estritamente necessárias para definir feixes. ${ }^{6}$ Antes de definir a noção final de cobertura (as topologias de Grothendieck), precisamos definir crivos.

Definição 2.3.3. Sejam $\mathcal{C}$ uma categoria pequena e $c \in \mathcal{C}_{0}$. Um subconjunto $S \subseteq \bigcup_{a \in \mathcal{C}_{0}} \mathcal{C}(a, c)$ é dito um crivo em $c$ se, para todo $g \in S$ e todo $f: b \rightarrow \operatorname{dom}(g)$ em $\mathcal{C}_{1}, g \circ f \in S$.

Ou seja, um crivo é um ideal à direita para a composição.

Definição 2.3.4. Sejam $\mathcal{C}$ uma categoria pequena, $c \in \mathcal{C}_{0}, S$ um crivo em $c$ e $f: a \rightarrow c$ em $\mathcal{C}_{1}$. O pullback de $S$ por $f$ é um novo crivo $f^{*} S$ em $a$ definido por:

$$
f^{*} S:=\bigcup_{b \in \mathcal{C}_{0}}\left\{g: b \rightarrow a \mathrm{em} \mathcal{C}_{1} \mid f \circ g \in S\right\}
$$

\footnotetext{
${ }^{6}$ Aliás, para definir feixes apenas, a única condição importante é a estabilidade por pullback; e seria possível ainda considerar uma forma mais fraca dela para categorias sem pullbacks, como é feito em [Joh02b] (capítulo C2). Escolhemos aqui apresentar a forma mais usual da definição, que é também mais próxima da originalmente usada por Grothendieck.
} 
Agora, se pensarmos em crivos em locales, veremos que crivos são exatamente os conjuntos hereditários. Mais uma vez, vale:

Proposição 2.3.5. Sejam $\mathcal{C}$ uma categoria pequena e $c \in \mathcal{C}_{0}$. Então, existe uma bijeção entre os crivos $S$ em c e os subfuntores $\tilde{S}$ de $\mathcal{C}(-, c)$.

De fato, dado um crivo $S$ em $c$, podemos definir o subfuntor $\tilde{S}$ de $\mathcal{C}(-, c)$ fazendo:

$$
\begin{array}{r}
\tilde{S}_{0}(a):=\{f: a \rightarrow c \mid f \in S\}, \text { para todo } a \in \mathcal{C}_{0} \\
\tilde{S}_{1}(g): \tilde{S}_{0}(a) \rightarrow \tilde{S}_{0}\left(a^{\prime}\right), \text { para toda } g: a^{\prime} \rightarrow a \text { em } \mathcal{C}_{1}^{o p}, \\
\text { onde } \tilde{S}_{1}(g)(f):=f \circ g: a^{\prime} \rightarrow c, \text { para toda } f: a \rightarrow c \text { em } S
\end{array}
$$

Por outro lado, dado um subfuntor $F$ de $\mathcal{C}(-, c)$, definimos o crivo em $c$ :

$$
S_{F}:=\bigcup_{a \in \mathcal{C}_{0}} F(a)
$$

Com isso, vale $\widetilde{S_{F}}=S$ e $S_{\tilde{F}}=F$.

Agora talvez chamar $f^{*} S$ de pullback do crivo $S$ por $f: a \rightarrow c$ faça mais sentido: o funtor correspondente ao pullback de um crivo é o pullback da inclusão $i_{\tilde{S}}: \tilde{S} \Rightarrow \mathcal{C}(-, c)$ e $\mathcal{C}(-, f): \mathcal{C}(-, a) \Rightarrow \mathcal{C}(-, c)$ :

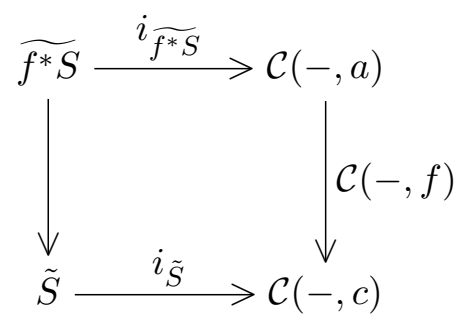

Definição 2.3.6. Seja $\mathcal{C}$ uma categoria pequena. Uma topologia de Grothendieck em $\mathcal{C}$ é uma função $J: \mathcal{C}_{0} \rightarrow \mathcal{P}\left(\mathcal{P}\left(\mathcal{C}_{1}\right)\right)$ tal que, para todo $c \in \mathcal{C}_{0}$ e para todo $S \in J(c), S$ é um crivo em $c$, e satisfaz:

1. identidade (ou crivo maximal): para todo $c \in \mathcal{C}_{0}, \bigcup_{a \in \mathcal{C}_{0}} \mathcal{C}(a, c) \in J(c)$;

2. mudança de base (ou estabilidade por pullback): dado $c \in \mathcal{C}_{0}$, para todo $S \in J(c)$, para toda $f: a \rightarrow c$ em $\mathcal{C}_{1}, f^{*} S \in J(a)$

3. caráter local (ou transitividade): dado $c \in \mathcal{C}_{0}$ e $S \in J(c)$, se $R$ é um crivo em $c$ tal que, 
para toda $f: a \rightarrow c$ em $S, f^{*} R$ é um crivo em $a$, então $R \in J(c)$.

Para fazer analogia com coberturas em locales, a primeira condição corresponde à ideia de que $\downarrow a$ é uma cobertura de $a$. A segunda condição corresponde à ideia que se $\left\{a_{i} \mid i \in I\right\}$ é uma cobertura de $a$ (isto é, $\bigvee_{i \in I} a_{i}=a$ ), então $\left\{a_{i} \wedge b \mid i \in I\right\}$ é uma cobertura de $a \wedge b$. Finalmente, a terceira condição correponde à ideia de se $\left\{a_{i} \mid i \in I\right\}$ é uma cobertura de $a$ e, para cada $i \in I$, $\left\{a_{i j} \mid j \in J_{i}\right\}$ é uma cobertura de $a_{i}$, então $\left\{a_{i j} \mid i \in I, j \in J_{i}\right\}$ é também uma cobertura de $a$.

Proposição 2.3.7. Sejam $\mathcal{C}$ uma categoria pequena e J uma topologia de Grothendieck em $\mathcal{C}$. Então, para todo $c \in \mathcal{C}_{0},(J(c), \subseteq)$ é um filtro no conjunto dos crivos sobre $c$.

Demonstração. Dado $c \in \mathcal{C}_{0}$, o crivo maximal sempre pertence a $J(c)$, por definição.

Sejam $S \in J(c)$ e $R$ um crivo. Se $S \subseteq R$, então para toda $f: b \rightarrow c$ em $S, f \in R$, $\operatorname{logo} f^{*} R=\bigcup_{a \in \mathcal{C}_{0}} \mathcal{C}(a, b)$ (pois $R$ é crivo). Pela primeira condição de topologia de Grothendieck, $f^{*} R \in J(b)$, e pela terceira condição, $R \in J(c)$.

Agora, sejam $S_{1}, S_{2} \in J(c)$. Para todos $f_{1}: b \rightarrow c$ em $S_{1}$ e $g: a \rightarrow b$ em $\mathcal{C}_{1}, f_{1} \circ g \in S_{1}$. Portanto, $f_{1} \circ g \in S_{2}$ se, e somente se, $f_{1} \circ g \in S_{1} \cap S_{2}$; ou seja, $f_{1}^{*} S_{2}=f_{1}^{*}\left(S_{1} \cap S_{2}\right)$. Assim, pela segunda condição de topologia de Grothendieck, $f_{1}^{*}\left(S_{1} \cap S_{2}\right) \in J(b)$, e, novamente usando a última condição, concluímos que $S_{1} \cap S_{2} \in J(c)$.

Proposição 2.3.8. Sejam $\mathcal{C}$ uma categoria pequena com pullbacks binários e cov uma pretopologia de Grothendieck. Então, a menor topologia de Grothendieck que contém cov é dada por:

$$
\begin{array}{r}
J_{\operatorname{cov}}(c):=\left\{S \subseteq \bigcup_{a \in \mathcal{C}_{0}} \mathcal{C}(a, c) \mid S \text { é crivo e existe } C=\left\{f_{i}: b_{i} \rightarrow c \mid i \in I\right\} \in \operatorname{cov}(c)\right. \\
\quad \text { tal que crivo }(C) \subseteq S\}
\end{array}
$$

para todo $c \in \mathcal{C}_{0}$, onde: $\operatorname{crivo}(C)=\left\{g \in \mathcal{C}_{1} \mid \exists i \in I, \exists h_{i}: \operatorname{dom}(g) \rightarrow b_{i}\left(g=f_{i} \circ h_{i}\right)\right\}$

Finalmente, pois, podemos definir:

Definição 2.3.9. Sejam $\mathcal{C}$ uma categoria pequena e $J$ uma topologia de Grothendieck em $\mathcal{C}$. Então, o par $(\mathcal{C}, J)$ é chamado de um sítio (pequeno).

Novamente, um prefeixe sobre um sítio $(\mathcal{C}, J)$ é simplesmente um funtor $F: \mathcal{C}^{o p} \rightarrow$ Set. Dessa forma: 
Definição 2.3.10. Sejam $(\mathcal{C}, J)$ um sítio e $F: \mathcal{C}^{o p} \rightarrow$ Set um prefeixe. $F$ é dito um feixe (de conjuntos) sobre $(\mathcal{C}, J)$ se, para todo $c \in \mathcal{C}_{0}$, para todo $\left\{f_{i}: a_{i} \rightarrow c \mid i \in I\right\} \in J(c)$ e para toda família $F$-compatível $\left\{s_{i} \in F_{0}\left(a_{i}\right) \mid i \in I\right\}$ (isto é, para todos $i, j \in I$ e para todas $g: b \rightarrow a_{i}, h: b \rightarrow a_{j}$ em $\mathcal{C}_{1}$ tais que $f_{i} \circ g=f_{j} \circ h$, temos $\left.F_{1}(g)\left(s_{i}\right)=F_{1}(h)\left(s_{j}\right)\right)$, existe um único $s \in F_{0}(c)$ tal que $F_{1}\left(f_{i}\right)(s)=s_{i}$, para todo $i \in I$. A categoria dos feixes sobre $(\mathcal{C}, J)$ e transformações naturais entre eles será denotada por $\mathbf{S h}(\mathcal{C}, J)$.

Definição 2.3.11. Sejam $(\mathcal{C}, J)$ um sítio e $F: \mathcal{C}^{o p} \rightarrow$ Set um prefeixe. $F$ é dito um prefeixe separado (ou um monoprefeixe) se, para todo $c \in \mathcal{C}_{0}$, para todo $\left\{f_{i}: a_{i} \rightarrow c \mid i \in I\right\} \in J(c)$ e para toda família $F$-compatível $\left\{s_{i} \in F_{0}\left(a_{i}\right) \mid i \in I\right\}$, existe no máximo um $s \in F_{0}(c)$ tal que $F_{1}\left(f_{i}\right)(s)=s_{i}$, para todo $i \in I$ (ou seja, exigimos apenas a unicidade, não a existência, da condição de feixe).

Consideremos então o caso de uma categoria com pullbacks binários, de forma que podemos usar pretopologias para obtermos uma condição mais familiar para feixes.

Primeiramente, sejam $\mathcal{C}$ uma categoria com pullbacks binários, cov uma pretopologia de Grothendieck em $\mathcal{C}$ e $F: \mathcal{C}^{o p} \rightarrow$ Set um prefeixe. Para $c \in \mathcal{C}_{0}$, considere o crivo $S=\left\{f_{i}:\right.$ $\left.a_{i} \rightarrow c \mid i \in I\right\} \in J_{\text {cov }}(c)$. Então, para toda família $F$-compatível $\left\{s_{i} \in F_{0}\left(a_{i}\right) \mid i \in I\right\}$, temos $F_{1}\left(f_{j}^{\prime}\right)\left(s_{i}\right)=F_{1}\left(f_{i}^{\prime}\right)\left(s_{j}\right)$, para todos $i, j \in I$, onde $\left(a_{i} \times_{c} a_{j}, f_{i}^{\prime}, f_{j}^{\prime}\right)$ é o pullback de $f_{i}$ e $f_{j}$ :

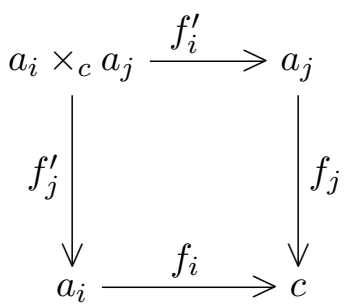

Aliás, toda família $F$-compatível é dessa forma: seja $\left\{s_{i} \in F_{0}\left(a_{i}\right) \mid i \in I\right\}$ uma família tal que $F_{1}\left(f_{j}^{\prime}\right)\left(s_{i}\right)=F_{1}\left(f_{i}^{\prime}\right)\left(s_{j}\right)$, para todos $i, j \in I$, e considere $g: b \rightarrow a_{i}, h: b \rightarrow a_{j}$ em $\mathcal{C}_{1}$ tais que $f_{i} \circ g=f_{j} \circ h$. Então, usando que o quadrado acima é um pullback, existe um único $\kappa: b \rightarrow a_{i} \times_{c} a_{j}$ tal que $f_{j}^{\prime} \circ \kappa=g$ e $f_{i}^{\prime} \circ \kappa=h$. Logo:

$$
\begin{aligned}
& F_{1}(g)\left(s_{i}\right)=F_{1}\left(f_{j}^{\prime} \circ \kappa\right)\left(s_{i}\right)=F_{1}(\kappa)\left(F_{1}\left(f_{j}^{\prime}\right)\left(s_{i}\right)\right)= \\
& \quad=F_{1}(\kappa)\left(F_{1}\left(f_{i}^{\prime}\right)\left(s_{j}\right)\right)=F_{1}\left(f_{i}^{\prime} \circ \kappa\right)\left(s_{j}\right)=F_{1}(h)\left(s_{i}\right)
\end{aligned}
$$

Teorema 2.3.12. Sejam $\mathcal{C}$ uma categoria com pullbacks binários, cov uma pretopologia de Grothendieck em $\mathcal{C}$ e $F: \mathcal{C}^{o p} \rightarrow$ Set um prefeixe. Então, são equivalentes: 
1. F é um feixe sobre $\left(\mathcal{C}, J_{\text {cov }}\right)$;

2. para todo $c \in \mathcal{C}_{0}$, para toda família $\left\{f_{i}: a_{i} \rightarrow c \mid i \in I\right\} \in \operatorname{cov}(c)$,

$$
F_{0}(c) \stackrel{d}{\longrightarrow} \prod_{i \in I} F_{0}\left(a_{i}\right) \stackrel{r}{\mathrm{e}} \underset{(i, j) \in I \times I}{\longrightarrow} \prod_{0} F_{0}\left(a_{i} \times{ }_{c} a_{j}\right)
$$

é um diagrama de equalizador, onde:

$$
\begin{aligned}
d(x) & =\left(F_{1}\left(f_{i}\right)(x)\right)_{i \in I} \\
r\left(\left(x_{i}\right)_{i \in I}\right) & =\left(F_{1}\left(f_{j}^{\prime}\right)\left(x_{i}\right)\right)_{(i, j) \in I \times I} \\
e\left(\left(x_{i}\right)_{i \in I}\right) & =\left(F_{1}\left(f_{i}^{\prime}\right)\left(x_{j}\right)\right)_{(i, j) \in I \times I}
\end{aligned}
$$

(no caso de F ser apenas um prefeixe separado, basta que d seja um mono)

Uma característica especial das coberturas em locales é que todo prefeixe representável é um feixe. Para sítios, isso nos leva à definição:

Definição 2.3.13. Seja $(\mathcal{C}, J)$ um sítio pequeno. A topologia $J$ é dita subcanônica se, para todo $c \in \mathcal{C}_{0}$, o funtor $\mathcal{C}(-, c)$ é um feixe sobre $(\mathcal{C}, J)$. A maior topologia subcanônica é chamada de topologia canônica.

Além das coberturas em locales, outro exemplo importante é a Topologia de Zariski ${ }^{7}$

Em categorias com pullbacks binários, as topologias subcanônicas correspondem às famílias epimórficas efetivas, isto é, famílias $\left\{f_{i}: a_{i} \rightarrow b \mid i \in I\right\}$ tais que, para toda família $\left\{g_{i}: a_{i} \rightarrow c \mid\right.$ $i \in I\}$ satisfazendo para todos $i, j \in I, g_{i} \circ e_{i}^{j}=g_{j} \circ e_{j}^{i}$, onde $e_{i}^{j}$ e $e_{j}^{i}$ são definidas pelo pullback:

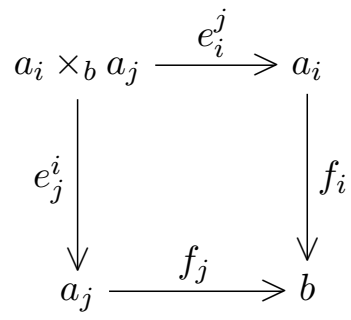

existe um único morfismo $g: b \rightarrow c$ tal que $g_{i}=g \circ f_{i}$, para todo $i \in I$.

\footnotetext{
${ }^{7}$ A Topologia de Zariski é uma topologia no espectro primo de um anel comutativo, e é bastante importante em geometria algébrica. Como referência, uma breve apresentação da Topologia de Zariski é feita na seção III.3 de [LM94].
} 
Nesse caso, a topologia canônica é dada pelas famílias estavelmente epimórficas efetivas: uma família $\left\{f_{i}: a_{i} \rightarrow b \mid i \in I\right\}$ é dita estavelmente epimórfica efetiva se para qualquer morfismo $h: b^{\prime} \rightarrow b$, a família $\left\{f_{i}^{\prime}: a_{i} \times_{b} b^{\prime} \rightarrow b^{\prime} \mid i \in I\right\}$, obtida pelo pullback:

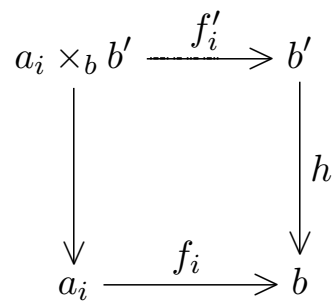

é também epimórfica efetiva. ${ }^{8}$

Semelhante ao caso para feixes sobre locales, como temos uma bijeção entre crivos e subfuntores do funtor hom, obtemos outra forma de definir feixes.

Proposição 2.3.14. Sejam $(\mathcal{C}, J)$ um sítio e $F: \mathcal{C}^{o p} \rightarrow$ Set um prefeixe. Para todo $S=\left\{f_{i}\right.$ : $\left.a_{i} \rightarrow c \mid i \in I\right\} \in J(c)$, com subfuntor $\tilde{S}$ de $\mathcal{C}(-, c)$ correspondente (pela proposição 2.3.5), existe uma bijeção entre as famílias $F$-compativeis $\left\{s_{i} \in F_{0}\left(a_{i}\right) \mid i \in I\right\}$ e as transformações naturais $\eta: \tilde{S} \Rightarrow F$.

De fato, novamente a família compatível é exatamente imagem da transformação natural. Agora, como um elemento $s \in F_{0}(c)$ corresponde, pelo Lema de Yoneda, a uma transformação natural $\mathcal{C}(-, c) \Rightarrow F$, mais uma vez obtemos:

Proposição 2.3.15. Sejam $(\mathcal{C}, J)$ um sítio e $F: \mathcal{C}^{o p} \rightarrow$ Set um prefeixe. Então, são equivalentes:

1. F é um feixe sobre $(\mathcal{C}, J)$;

2. para todos $c \in \mathcal{C}_{0}$ e $S \in J(c)$ com $\tilde{S}$ subfuntor de $\mathcal{C}(-, c)$, toda transformação natural $\eta: \tilde{S} \Rightarrow F$ pode ser estendida unicamente a uma transformação natural $\tilde{\eta}: \mathcal{C}(-, c) \Rightarrow F$.

Definição 2.3.16. Um topos de Grothendieck $\mathcal{E}$ é uma categoria (localmente pequena) equivalente à categoria $\mathbf{S h}(\mathcal{C}, J)$, para um sítio $(\mathcal{C}, J)$.

Os morfismos de topoi de Grothendieck são definidos de forma semelhante aos morfismos de locales:

\footnotetext{
${ }^{8}$ Para mais detalhes, ver proposição 1.1.9 de [MR77].
} 
Definição 2.3.17. Sejam $\mathcal{E}, \mathcal{E}^{\prime}$ topoi de Grothendieck. Um morfismo geométrico é um par de funtores $\left(\varphi_{*}, \varphi^{*}\right): \mathcal{E} \rightarrow \mathcal{E}^{\prime}, \operatorname{com} \varphi_{*}: \mathcal{E} \rightarrow \mathcal{E}^{\prime}$ e $\varphi^{*}: \mathcal{E}^{\prime} \rightarrow \mathcal{E}$, tal que $\varphi^{*} \dashv \varphi_{*}$ e $\varphi^{*}$ preserva limites finitos.

Observe que a adjunção garante que $\varphi^{*}$ (geralmente chamada de imagem inversa) preserve todos os colimites, e que $\varphi_{*}$ (geralmente chamada de imagem direta) preserve todos os limites.

Exemplo 2.3.18. Seja $f: L \rightarrow L^{\prime}$ um morfismo de locales. Defina $\varphi_{*}: \operatorname{Sh}(L) \rightarrow \operatorname{Sh}\left(L^{\prime}\right)$ fazendo, para cada feixe $F: L^{o p} \rightarrow$ Set, $\left(\varphi_{*}\right)_{0}(F)=F \circ f^{*}: L^{\prime o p} \rightarrow$ Set. $\varphi_{*}$ possui um adjunto à esquerda $\varphi^{*}$, e o par $\left(\varphi_{*}, \varphi^{*}\right)$ é um morfismo geométrico ${ }^{9}$.

Exemplo 2.3.19. Seja $(\mathcal{C}, J)$ um sítio. Para cada prefeixe $F: \mathcal{C}^{o p} \rightarrow$ Set, definimos o funtor $F^{+}: \mathcal{C}^{o p} \rightarrow$ Set fazendo:

$$
F^{+}(c):=\underset{R \in J(c)}{\operatorname{colim}} \operatorname{Nat}(\tilde{R}, F), \text { para todo } c \in \mathcal{C}_{0}
$$

(ou seja, o colimite do funtor obtido por composição de $\operatorname{Set}^{\mathcal{C}^{o p}}(-, F)$ com a inclusão $\widetilde{J(c)} \hookrightarrow$ $\left.\mathbf{S e t}^{\mathcal{C}^{o p}}\right)$. Essa construção se estende a um funtor $(-)^{+}: \mathbf{S e t}^{\mathcal{C}^{o p}} \rightarrow \mathbf{S e t}^{\mathcal{C o p}^{\circ}}$ que preserva limites finitos. Além disso, denotando $\eta: i d_{\text {Set }^{c^{o p}}} \Rightarrow(-)^{+}$, vale: $F^{+}$é sempre prefeixe separado; se $F$ é prefeixe separado, então $\eta_{F}$ é mono; se $F$ é prefeixe separado, então $F^{+}$é feixe; se $F$ é feixe, então $\eta_{F}$ é iso.

Assim, podemos definir o funtor $a:=(-)^{++}:=(-)^{+} \circ(-)^{+}: \operatorname{Set}^{\mathcal{C}^{o p}} \rightarrow \mathbf{S h}(\mathcal{C}, J)$, chamado de funtor feixe associado (ou feixificação). Tal funtor preserva limites finitos ${ }^{10}$, e, chamando de $i: \mathbf{S h}(\mathcal{C}, J) \hookrightarrow \mathbf{S e t}^{\mathcal{C}^{o p}}$ a inclusão, também vale $a \dashv i$. Agora, note que $\mathbf{S e t}^{\mathcal{C}^{o p}}$ é um topos de Grothendieck: considere a topologia de Grothendieck dada por $J^{\prime}(c)=\left\{S_{\mathcal{C}(-, c)}\right\}$, para cada $c \in \mathcal{C}_{0}$, de forma que é imediato da proposição 2.3 .15 que todo prefeixe é um feixe sobre $\left(\mathcal{C}, J^{\prime}\right)$. Assim, obtemos que $(a, i): \mathbf{S e t}^{\mathcal{C}^{o p}} \rightarrow \mathbf{S h}(\mathcal{C}, J)$ forma um morfismo geométrico. ${ }^{11}$

Existe também uma caracterização axiomática dos topoi de Grothendieck dada pelo teorema de Giraud, como categorias satisfazendo certas propriedades de tamanho, de existência de limites e de interação entre limites (e colimites). Recomenda-se consultar o teorema 3.6.1 de [Bor08a] ou o teorema C2.2.8 de [Joh02b] para sua formulação precisa e demonstração.

\footnotetext{
${ }^{9} \mathrm{~A}$ existência desse adjunto decorre do teorema do funtor adjunto (ver 1.7.7). Porém, existe também uma forma mais fácil de obtê-lo usando feixes geométricos: dado $g \in \mathbf{L} \mathbf{H}_{l o c} \downarrow L^{\prime}$, defina $\varphi^{*}(g)$ como o (feixe funtorial correspondente ao) pullback de $g$ ao longo de $f$.

${ }^{10}$ Pois limites finitos comutam com colimites filtrantes em topoi de Grothendieck.

${ }^{11} \mathrm{~A}$ construção detalhada do funtor e a verificação de suas propriedades pode ser consultada na seção 3.3 de [Bor08c] ou na seção III.5 de [LM94].
} 
Para finalizar, apenas registremos um fato sobre objetos números naturais:

Proposição 2.3.20. Todo topos de Grothendieck possui objeto números naturais.

O objeto números naturais de um topos de Grothendieck é obtido dos números naturais em Set. Mais precisamente, pode-se mostrar que se $\left(\varphi_{*}, \varphi^{*}\right): \mathcal{E} \rightarrow \mathcal{E}^{\prime}$ é um morfismo geométrico entre dois topoi (não necessariamente de Grothendieck, aliás) e $\mathcal{E}^{\prime}$ possui objeto números naturais $\mathbb{N}_{\mathcal{E}^{\prime}}$, então $\varphi^{*}: \mathcal{E}^{\prime} \rightarrow \mathcal{E}$ o preserva, isto é, $\varphi_{0}^{*}\left(\mathbb{N}_{\mathcal{E}^{\prime}}\right)$ é objeto números naturais em $\mathcal{E}$. Com isso, como para todo topos de Grothendieck $\mathcal{T}$ sempre existe um morfismo geométrico $\left(\varphi_{*}, \varphi^{*}\right): \mathcal{T} \rightarrow$ Set e existe objeto números naturais em Set, $\mathcal{T}$ também possui objeto números naturais. Ver o corolário 8.1.5 de [Bor08c] para mais detalhes. 


\section{Capítulo 3}

\section{Topos elementar}

Apresentamos, neste capítulo, os topoi elementares: com inspiração nos topoi de Grothendieck do capítulo anterior, um topos elementar é definido como uma categoria que terá certas propriedades categoriais em comum com Set.

Existem duas formas de definir um topos elementar: uma delas é mais concisa e usa o objeto partes, um objeto que captura a noção do conjunto partes em Set. Outra, útil por ressaltar propriedades importantes do topos (principalmente para a lógica), usa os conceitos de categoria cartesianamente fechada e classificador de subobjeto. Adotamos a segunda como definição principal, por isso iniciamos o capítulo apresentando categorias cartesianamente fechadas (categorias onde definimos uma noção de exponenciação) e o classificador de subobjeto (uma forma de generalizar a ideia do "conjunto de valores verdade", inspirando-se nas funções características em Set). Na seção seguinte, definimos um topos elementar e sua descrição equivalente usando objeto partes, e introduzimos as duas noções de morfismos de topoi: funtores lógicos e morfismos geométricos.

Passamos então a apresentar diversos resultados e exemplos principais de topoi; e em seguida definimos alguns tipos de topoi e listamos algumas de suas propriedades. Para finalizar o capítulo, mostramos como podemos generalizar a noção de topologia e feixes no contexto dos topoi de Grothendieck para um topos elementar.

Como bibliografia para esse tópico, recomendamos os capítulos 2 e 5 de [BW85], o capítulo 5 de [Bor08c] (com categorias cartesianamente fechadas feitas na seção 6.1 de [Bor08b]), e os capítulos A1 e A2 de [Joh02a]. Outras referências são [Gol79] (capítulos 4 e 5) e [Mc195] (parte III), ambas com um foco maior para a construção da lógica de topos. 


\subsection{Categorias cartesianamente fechadas}

A ideia por trás de definir a exponenciação numa categoria é fazer com que um morfismo definido num produto corresponda a um morfismo definido em apenas um de seus fatores. Para a formulação precisa, usamos funtores adjuntos:

Definição 3.1.1. Seja $\mathcal{C}$ uma categoria localmente pequena com produtos binários. Para cada $b \in \mathcal{C}_{0}$, considere o funtor $-\times b: \mathcal{C} \rightarrow \mathcal{C}$, dado por:

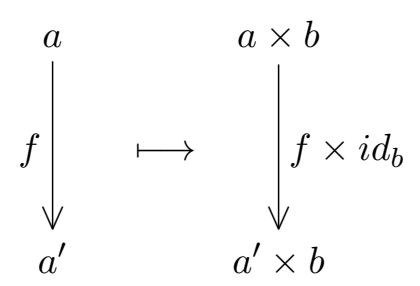

para todos $a, a^{\prime} \in \mathcal{C}_{0}$ e $f \in \mathcal{C}_{1}$. Dizemos que $\mathcal{C}$ é cartesianamente fechada se, para todo $b \in \mathcal{C}_{0}$, o funtor $-\times b$ possui um adjunto à direita.

É comum denotarmos esse adjunto por $(-)^{b}: \mathcal{C} \rightarrow \mathcal{C}$, chamado de funtor exponenciação, de forma que para todos $a, b, c \in \mathcal{C}_{0}$ temos a bijeção natural:

$$
\mathcal{C}(a \times b, c) \cong \mathcal{C}\left(a, c^{b}\right)
$$

Uma forma mais elementar de apresentar a exponenciação é usar a counidade da adjunção: para cada $c \in \mathcal{C}_{0}$, chamemos a counidade $e v_{c}: c^{b} \times b \rightarrow c$ de avaliação. Assim, para toda flecha $h: a \times b \rightarrow c$, existe uma única flecha $\exp (h): a \rightarrow c^{b}$, chamada de transposta exponencial, tal que o diagrama abaixo é comutativo:

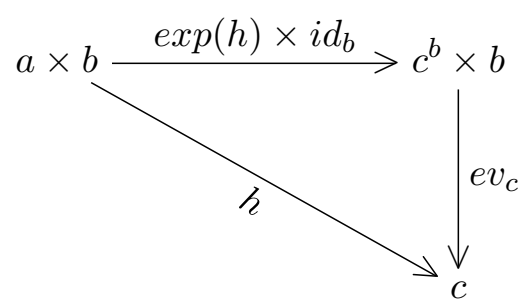

Com isso, também podemos definir a exponencial de duas flechas: sejam $f: a \rightarrow a^{\prime}$ e $g: b^{\prime} \rightarrow b$ em $\mathcal{C}_{1}$. Definimos a flecha $f^{g}: a^{b} \rightarrow a^{\prime b^{\prime}}$ como a transposta exponencial da flecha $e v_{a^{\prime}} \circ f^{b} \times g$ :

$$
a^{b} \times b^{\prime} \stackrel{f^{b} \times g}{\longrightarrow} a^{b} \times b \stackrel{e v_{a^{\prime}}}{\longrightarrow} a^{\prime}
$$

Exemplo 3.1.2. Como as notações sugerem, Set e Cat são categorias cartesianamente fechadas 
onde as exponenciações são dadas, respectivamente, por $b^{a}$ (o conjunto das funções $a \rightarrow b$ ) e $\mathcal{B}^{\mathcal{A}}$ (o conjunto dos funtores $\mathcal{A} \rightarrow \mathcal{B}$ ).

Façamos o caso em Set com mais detalhes: a flecha avaliação em Set é dada por, como o nome sugere, $e v_{C}^{B}: C^{B} \times B \rightarrow C$ com $e v_{C}^{B}(f, b)=f(b)$, para toda $f: B \rightarrow C$ e $b \in B$. Agora, para toda flecha $h: A \times B \rightarrow C$, queremos definir a transposta exponencial $\exp (h): A \rightarrow C^{B}$. A ideia é que podemos definir uma função $B \rightarrow C$ a partir de $h$ se fixarmos um $a \in A$ e observarmos a função obtida fazendo a variável percorrer apenas $B$ : para cada $a \in A$, defina a função $h_{a}: B \rightarrow C$ como $h_{a}(b)=h(a, b)$, para todo $b \in B$.

Com isso, defina $\exp (h): A \rightarrow C^{B} \operatorname{como} \exp (h)(a)=h_{a}$, para todo $a \in A$, de forma que, para todo $(a, b) \in A \times B$ :

$$
e v_{C}^{B}\left(\exp (h)(a), i d_{B}(b)\right)=e v_{C}^{B}\left(h_{a}, b\right)=h_{a}(b)=h(a, b)
$$

Agora, para unicidade, se uma $g: A \rightarrow C^{B}$ também satisfaz

$$
e v_{C}^{B}\left(g(a), i d_{B}(b)\right)=e v_{C}^{B}(g(a), b)=g(a)(b)=h(a, b)
$$

para todos $(a, b) \in A \times B$, então $g(a)(b)=h_{a}(b)$. Logo, $g(a)=h_{a}$, para todo $a \in A$, e $g=\exp (h)$.

Exemplo 3.1.3. Se $(H, \leq)$ é uma álgebra de Heyting, então, considerada como uma categoria, é cartesianamente fechada. No caso, como o produto corresponde ao ínfimo, a exponenciação é dada pela implicação: para todos $a, b, c \in H$,

$$
H(a \wedge b, c) \cong H(a, b \rightarrow c)
$$

Proposição 3.1.4. Seja $\mathcal{C}$ uma categoria cartesianamente fechada. Então:

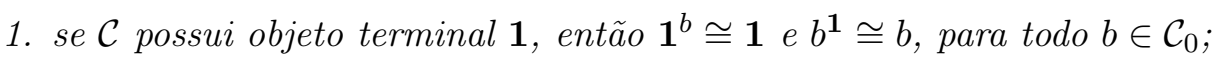

2. para todos $a, b, c \in \mathcal{C}_{0},(a \times b)^{c} \cong a^{c} \times b^{c}$;

3. se $\mathcal{C}$ possui objeto terminal $\mathbf{1}$ e inicial $\mathbf{0}$, então $b^{\mathbf{0}} \cong \mathbf{1}$, para todo $b \in \mathcal{C}_{0}$;

4. se $\mathcal{C}$ possui coprodutos binários, $a^{b} \amalg^{c} \cong a^{b} \times a^{c}$;

5. objetos iniciais são estritos, isto é, se $\mathcal{C}$ possui objeto inicial $\mathbf{0}$ e existe flecha $f: a \rightarrow \mathbf{0}$ em $\mathcal{C}_{1}$, então $a \cong \mathbf{0}$. 
A verificação desses resultados é fácil usando a bijeção natural $\mathcal{C}(a \times b, c) \cong \mathcal{C}\left(a, c^{b}\right)$ e o corolário 1.3.11 do Lema de Yoneda (além de propriedades de produto e coproduto, é claro). Por exemplo, no primeiro item, para todo $d \in \mathcal{C}_{0}$ temos

$$
\mathcal{C}\left(d, \mathbf{1}^{b}\right) \cong \mathcal{C}(d \times b, \mathbf{1}) \cong \mathcal{C}(d, \mathbf{1})
$$

donde $\mathbf{1}^{b} \cong \mathbf{1}$. Similarmente, obtemos $b^{\mathbf{1}} \cong b$ fazendo:

$$
\mathcal{C}\left(d, b^{\mathbf{1}}\right) \cong \mathcal{C}(d \times \mathbf{1}, b) \cong \mathcal{C}(d, b)
$$

Para finalizar essa seção, retomemos objetos números naturais. Em categorias cartesianamente fechadas, objetos números naturais permitem fazer um teorema da recursão generalizado:

Proposição 3.1.5. Recursão com parâmetros. Sejam $\mathcal{C}$ uma categoria cartesianamente fechada com objeto terminal 1 e $\left(\mathbb{N}_{\mathcal{C}}, 0_{\mathcal{C}}, s\right)$ um objeto números naturais em $\mathcal{C}$. Então, para todos $a, b \in \mathcal{C}_{0}$ e para todas flechas $g: a \rightarrow b$ e $h: a \times \mathbb{N}_{\mathcal{C}} \times b \rightarrow b$ em $\mathcal{C}_{1}$, existe uma única flecha $f: a \times \mathbb{N}_{\mathcal{C}} \rightarrow b$ tal que o diagrama abaixo é comutativo:

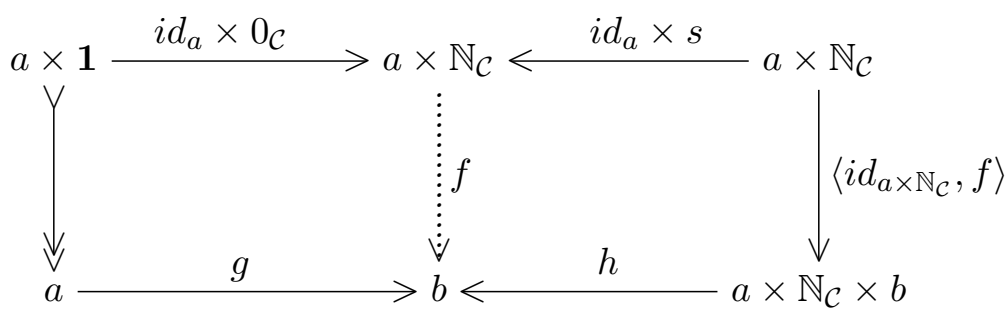

Intuitivamente, o quadrado esquerdo do diagrama diz que $f(x, 0)=g(x)$ e o lado direito diz que $f(x, n+1)=h(x, n, f(x, n))$. Façamos a demonstração desse resultado para ilustrar como se trabalha com categorias cartesianamente fechadas num nível mais técnico.

Demonstração. Primeiramente façamos o caso para $a=\mathbf{1}$ (e já omitamos os isomorfismos $d \times \mathbf{1} \cong$ $1 \times d \cong d$, para todo $d \in \mathcal{C})$. Como $\left(\mathbb{N}_{\mathcal{C}}, 0_{\mathcal{C}}, s\right)$ é objeto números naturais, existe uma única $k: \mathbb{N}_{\mathcal{C}} \rightarrow \mathbb{N}_{\mathcal{C}} \times b$ tornando o diagrama abaixo comutativo:

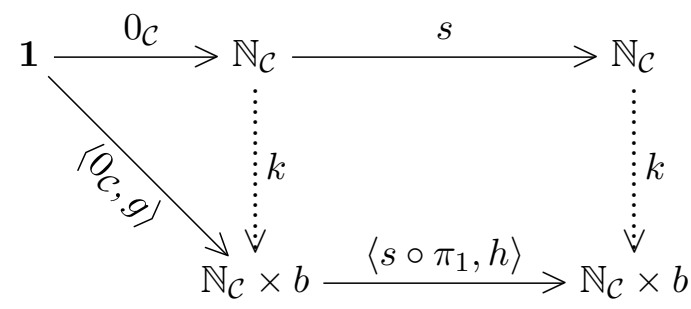


onde $\pi_{1}, \pi_{2}$ denotam as projeções do produto $\mathbb{N}_{\mathcal{C}} \times b$. Note que, dessa forma, a flecha $\pi_{1} \circ k$ : $\mathbb{N}_{\mathcal{C}} \rightarrow \mathbb{N}_{\mathcal{C}}$ satisfaz:

$$
\begin{gathered}
\pi_{1} \circ k \circ 0_{\mathcal{C}}=\pi_{1} \circ\left\langle 0_{\mathcal{C}}, g\right\rangle=0_{\mathcal{C}} \\
\pi_{1} \circ k \circ s=\pi_{1} \circ\left\langle s \circ \pi_{1}, h\right\rangle \circ k=s \circ \pi_{1} \circ k
\end{gathered}
$$

como na definição do objeto números naturais. Mas $i d_{\mathbb{N}_{\mathcal{C}}}: \mathbb{N}_{\mathcal{C}} \rightarrow \mathbb{N}_{\mathcal{C}}$ também satisfaz essas identidades; logo, por unicidade, $\pi_{1} \circ k=i d_{\mathbb{N}_{\mathcal{C}}}$.

Com isso, defina $f=\pi_{2} \circ k: \mathbb{N}_{\mathcal{C}} \rightarrow b$. Primeiramente, do diagrama acima temos $k \circ 0_{\mathcal{C}}=$ $\left\langle 0_{\mathcal{C}}, g\right\rangle$, portanto:

$$
f \circ 0_{\mathcal{C}}=\pi_{2} \circ k \circ 0_{\mathcal{C}}=\pi_{2} \circ\left\langle 0_{\mathcal{C}}, g\right\rangle=g
$$

Do mesmo diagrama também obtemos $\left\langle s \circ \pi_{1}, h\right\rangle \circ k=k \circ s$, ou seja, $h \circ k=\pi_{2} \circ k \circ s$. Assim, usando que $\pi_{1} \circ k=i d_{\mathbb{N}_{\mathcal{C}}}$ vale:

$$
h \circ\left\langle i d_{\mathbb{N}_{\mathcal{C}}}, f\right\rangle=h \circ\left\langle\pi_{1} \circ k, \pi_{2} \circ k\right\rangle=h \circ k=\pi_{2} \circ k \circ s=f \circ s
$$

de forma que obtemos exatamente a comutatividade do diagrama do enunciado para $a=\mathbf{1}$.

Para a unicidade, seja $f^{\prime}: \mathbb{N}_{\mathcal{C}} \rightarrow b$ tal que $f^{\prime} \circ 0_{\mathcal{C}}=g$ e $f^{\prime} \circ s=h \circ\left\langle i d_{\mathbb{N}_{\mathcal{C}}}, f^{\prime}\right\rangle$. Então, temos:

$$
\begin{gathered}
\left\langle i d_{\mathbb{N}_{\mathcal{C}}}, f^{\prime}\right\rangle \circ 0_{\mathcal{C}}=\left\langle 0_{\mathcal{C}}, f^{\prime} \circ 0_{\mathcal{C}}\right\rangle=\left\langle 0_{\mathcal{C}}, g\right\rangle \\
\left\langle s \circ \pi_{1}, h\right\rangle \circ\left\langle i d_{\mathbb{N}_{\mathcal{C}}}, f^{\prime}\right\rangle=\left\langle s \circ i d_{\mathbb{N}_{\mathcal{C}}}, h \circ\left(\left\langle i d_{\mathbb{N}_{\mathcal{C}}}, f^{\prime}\right\rangle\right)\right\rangle=\left\langle s, f^{\prime} \circ s\right\rangle=\left\langle i d_{\mathbb{N}_{\mathcal{C}}}, f^{\prime}\right\rangle \circ s
\end{gathered}
$$

Portanto, pela unicidade de $k$, temos $k=\left\langle i d_{\mathbb{N}_{\mathcal{C}}}, f^{\prime}\right\rangle$, donde $f=\pi_{2} \circ k=f^{\prime}$.

Podemos reduzir o caso geral a esse usando o fato da categoria ser cartesianamente fechada (mais uma vez, os isomorfismos no produto com o terminal serão omitidos). Sejam $\pi_{1}^{\prime}, \pi_{2}^{\prime}, \pi_{3}^{\prime}$ as projeções do produto $\mathbb{N}_{\mathcal{C}} \times b^{a} \times a$, e $\pi_{23}^{\prime}: \mathbb{N}_{\mathcal{C}} \times b^{a} \times a \rightarrow b^{a} \times a$ a projeção nas duas últimas coordenadas. Considere então as transpostas exponenciais $\exp (g): \mathbf{1} \rightarrow b^{a}$ e $\exp (\tilde{h}): \mathbb{N}_{\mathcal{C}} \times b^{a} \rightarrow$ $b^{a}$, onde chamamos $\tilde{h}:=h \circ\left\langle\pi_{3}^{\prime}, \pi_{1}^{\prime}, e v_{b} \circ \pi_{23}^{\prime}\right\rangle$. Pelo caso $a=\mathbf{1}$ acima, existe uma única $\hat{\kappa}: \mathbb{N}_{\mathcal{C}} \rightarrow b^{a}$ tornando o diagrama comutativo:

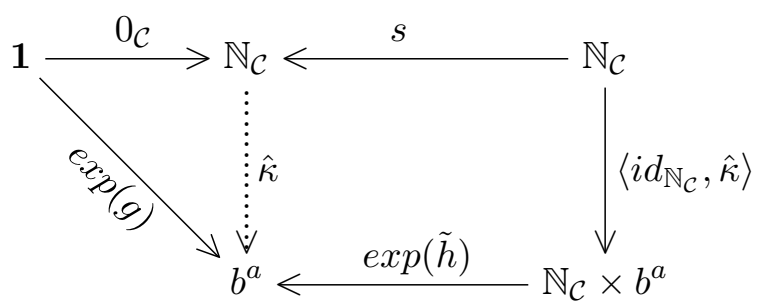


Com isso, seja $\kappa: \mathbb{N}_{\mathcal{C}} \times a \rightarrow b$ correspondente a $\hat{\kappa}$, isto é, $\hat{\kappa}=\exp (\kappa)$. Agora, não é difícil ver que, chamando de $\rho$ o iso $\mathbb{N}_{\mathcal{C}} \times a \cong a \times \mathbb{N}_{\mathcal{C}}$, vale:

$$
\left\langle\pi_{3}^{\prime}, \pi_{1}^{\prime}, e v_{b} \circ \pi_{23}^{\prime}\right\rangle \circ\left(\left\langle i d_{\mathbb{N}_{\mathcal{C}}}, \hat{\kappa}\right\rangle \times i d_{a}\right)=\langle\rho, \kappa\rangle
$$

(basta pensar em como cada flecha age em cada componente do produto). Assim, temos o diagrama comutativo:

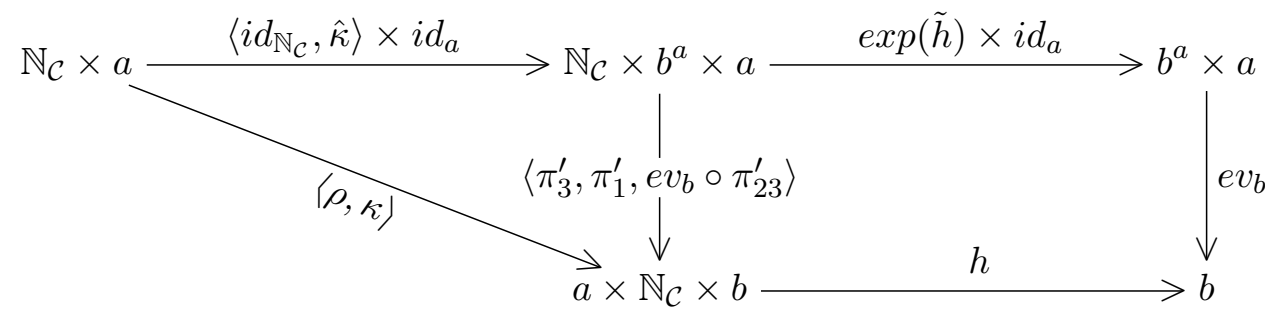

E, pela unicidade da transposta exponencial, $\exp (\tilde{h}) \circ\left\langle i d_{\mathbb{N}_{\mathcal{C}}}, \hat{\kappa}\right\rangle=\exp (h \circ\langle\rho, \kappa\rangle)$.

Além disso, é imediato que ambos os diagramas abaixo são comutativos:
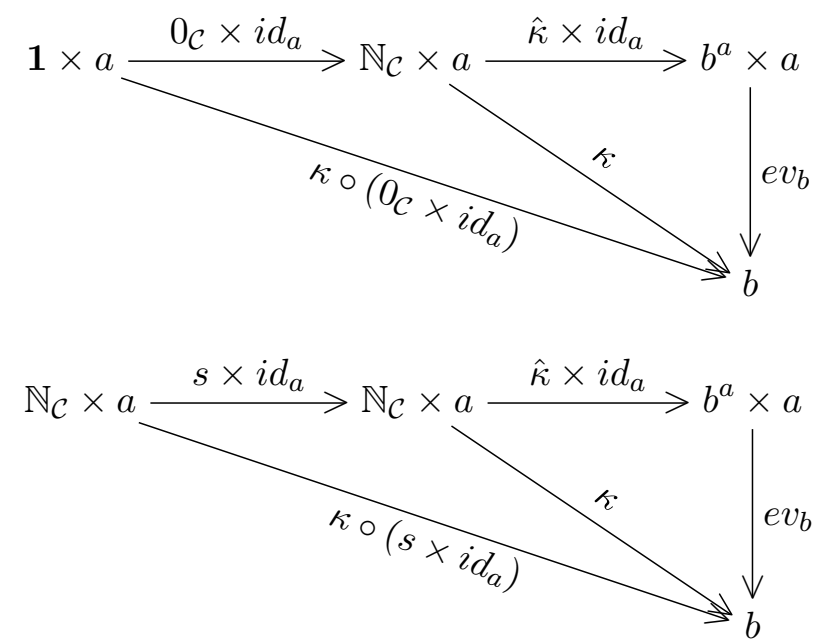

Donde, novamente pela unicidade das transpostas exponenciais, $\hat{\kappa} \circ 0_{\mathcal{C}}=\exp \left(\kappa \circ\left(0_{\mathcal{C}} \times i d_{a}\right)\right)$ e $\hat{\kappa} \circ s=\exp \left(\kappa \circ\left(s \times i d_{a}\right)\right)$. Ou seja, o diagrama (1) nos fornece as seguintes igualdades:

$$
\begin{gathered}
\exp (g)=\hat{\kappa} \circ 0_{\mathcal{C}}=\exp \left(\kappa \circ\left(0_{\mathcal{C}} \times i d_{a}\right)\right) \\
\exp (h \circ\langle\rho, \kappa\rangle)=\exp (\tilde{h}) \circ\left\langle i d_{\mathbb{N}_{\mathcal{C}}}, \hat{\kappa}\right\rangle=\hat{\kappa} \circ s=\exp \left(\kappa \circ\left(s \times i d_{a}\right)\right)
\end{gathered}
$$

Portanto, concluímos que $g=\kappa \circ\left(0_{\mathcal{C}} \times i d_{a}\right)$ e $h \circ\langle\rho, \kappa\rangle=\kappa \circ\left(s \times i d_{a}\right)$. Finalmente, definindo $f=\kappa \circ \rho^{-1}$, obtemos:

$$
g=\kappa \circ\left(0_{\mathcal{C}} \times i d_{a}\right)=f \circ \rho \circ\left(0_{\mathcal{C}} \times i d_{a}\right)=f \circ\left(i d_{a} \times 0_{\mathcal{C}}\right)
$$




$$
\begin{aligned}
& h \circ\left\langle i d_{a \times \mathbb{N}_{\mathcal{C}}}, f\right\rangle=h \circ\left\langle\rho \circ \rho^{-1}, \kappa \circ \rho^{-1}\right\rangle=h \circ\langle\rho, \kappa\rangle \circ \rho^{-1}= \\
& \quad=\kappa \circ\left(s \times i d_{a}\right) \circ \rho^{-1}=f \circ \rho \circ\left(s \times i d_{a}\right) \circ \rho^{-1}=f \circ\left(i d_{a} \times s\right)
\end{aligned}
$$

o que corresponde à comutatividade do diagrama do enunciado.

\subsection{Classificador de subobjetos}

Os classificadores de subobjeto são definidos de maneira a adaptar a noção das funções características em Set para um topos: a ideia é que cada subobjeto de um objeto $a$ corresponda a um morfismo de $a$ a um objeto especial.

Definição 3.2.1. Seja $\mathcal{C}$ uma categoria com pullbacks. Um classificador de subobjetos em $\mathcal{C}$ é um mono universal $\top: u \longmapsto \Omega$, isto é, para todo mono $m: a \longmapsto b$, existe um único $\chi_{m}: b \rightarrow \Omega$ tal que o diagrama abaixo é um quadrado de pullback:

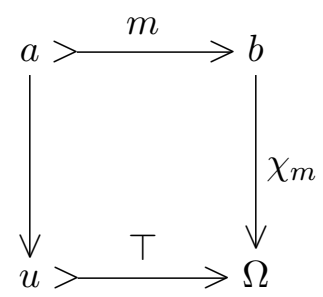

É comum chamar $\chi_{m}$ de flecha característica de $m$.

Exemplo 3.2.2. Podemos definir o classificador de subobjetos em Set como a função $T:\{*\} \rightarrow$ $\{0,1\}$ tal que $\top(*)=1$. Assim, para cada mono $f: S \hookrightarrow X$, a flecha característica é dada pela função característica $\chi_{f}:=\chi_{S}: X \rightarrow\{0,1\}$ :

$$
\chi_{S}(x)= \begin{cases}1 & , \text { se } x \in S \\ 0 & , \text { se } x \notin S\end{cases}
$$

É fácil ver que isso torna o diagrama da flecha característica um pullback em Set: lembrando o exemplo 1.4.9, temos, por definição,

$$
S=\left\{x \in X \mid \chi_{S}(x)=1\right\}=\left\{(*, x) \in\{*\} \times X \mid \chi_{S}(x)=\top(*)\right\}
$$

Em relação à unicidade, seja $g: X \rightarrow\{0,1\}$ tornando o diagrama um pullback. Então, como 
acima, temos:

$$
S=\{(*, x) \in\{*\} \times X \mid g(x)=\top(*)\}=\{x \in X \mid g(x)=1\}
$$

Logo, $g(x)=1=\chi_{S}(x)$ para todo $x \in S$, e $g(x)=0=\chi_{S}(x)$ para $x \in X \backslash S$. Ou seja, $g=\chi_{S}$ e a flecha característica é única.

Proposição 3.2.3. Seja $\mathcal{C}$ uma categoria com classificador de subobjetos $\top: u \longmapsto \Omega$. Então:

1. u é um objeto terminal;

2. se $T^{\prime}: u^{\prime} \longmapsto \Omega^{\prime}$ é classificador de subobjeto, então $\Omega \cong \Omega^{\prime}$;

3. sejam $A \in \mathcal{C}_{0}$ e $f, g \in M o n o(A)$. Então, $[f]=[g]$ se, e somente se, $\chi_{f}=\chi_{g}$;

4. todo mono em $\mathcal{C}$ é regular, e $\mathcal{C}$ é uma categoria balanceada (isto é, se uma flecha é um mono e um epi, então é um iso).

Demonstração. 1. Para todo $a \in \mathcal{C}_{0}$, a flecha $i d_{a}$ é mono, logo existe uma única $\chi_{i d_{a}}$ tal que o diagrama abaixo é um pullback:

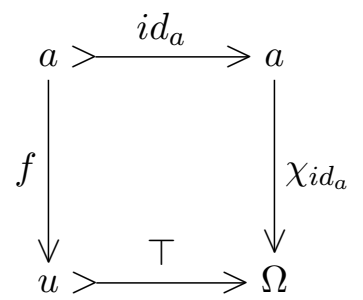

Assim, seja $g: a \rightarrow u$ em $\mathcal{C}_{1}$. Então, pela unicidade da flecha característica, temos $\top \circ g=\chi_{i d_{a}}=\top \circ f$, e como $\top$ é mono, concluímos que $g=f$. Ou seja, existe uma única flecha $a \rightarrow u$, e $u$ é objeto terminal.

2. Por definição, temos que os quadrados nos diagramas abaixo são pullbacks, de forma que, pelo lema do pullback, os retângulos também o são:
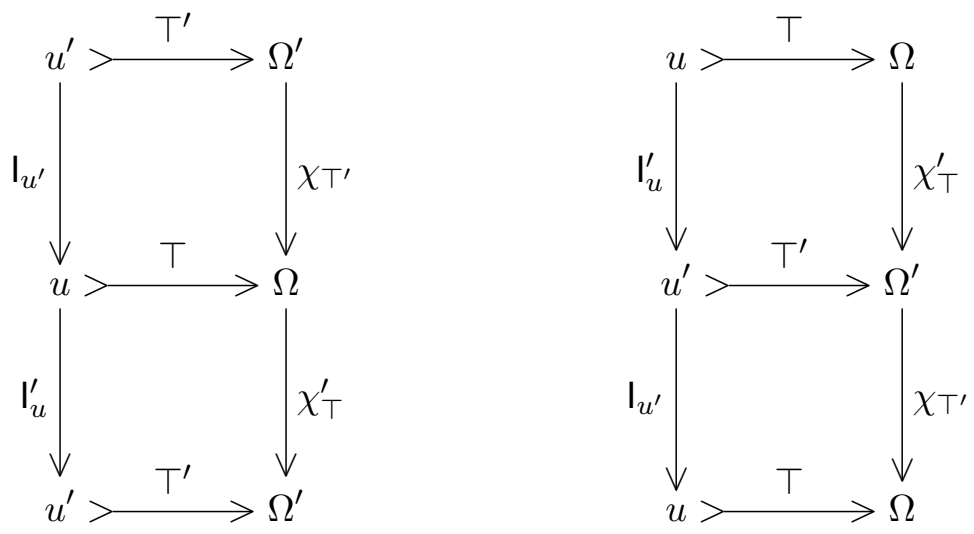
Assim, pela unicidade das flechas características, temos $\chi_{\top^{\prime}}^{\prime} \circ \chi_{T^{\prime}}=\chi_{\top^{\prime}}^{\prime}=i d_{\Omega^{\prime}}$ e $\chi_{T^{\prime}} \circ \chi_{\top}^{\prime}=$ $\chi_{\top}=i d_{\Omega}$, donde $\Omega \cong \Omega^{\prime}$.

3. Sejam $f: a \longmapsto A$ e $g: b \longmapsto A$. Então, $(\Rightarrow)$ por hipótese, existe iso $\kappa: b \rightarrow a$ tal que $f \circ \kappa=g$. Mostremos que o diagrama abaixo é um pullback:

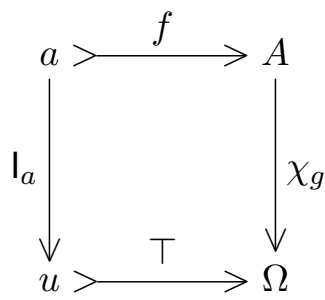

de forma que, por unicidade da flecha característica, temos $\chi_{f}=\chi_{g}$.

Primeiramente, usando o fato de $u$ ser terminal e a definição de $\chi_{g}$, temos:

$$
\top \circ \mathrm{I}_{a}=\top \circ \mathrm{I}_{b} \circ \kappa^{-1}=\chi_{g} \circ g \circ \kappa^{-1}=\chi_{g} \circ f
$$

e o diagrama é, de fato, comutativo.

Agora, seja $h: c \rightarrow A$ tal que $\chi_{g} \circ h=\top \circ \mathrm{I}_{c}$. Devemos achar $\rho: c \rightarrow a$ única que torna o diagrama comutativo:

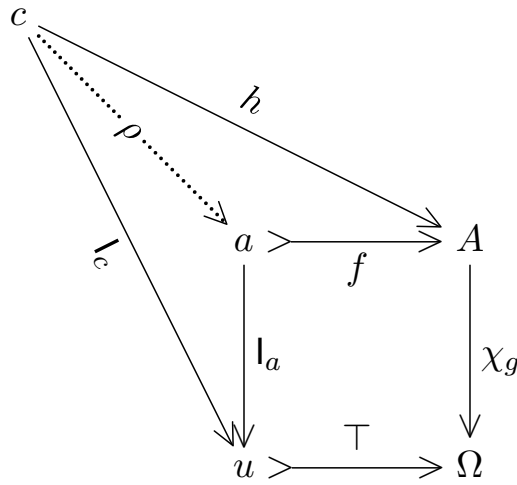

Pelo pullback da definição de $\chi_{g}$, temos que existe única $\rho^{\prime}: c \rightarrow b$ tal que $g \circ \rho^{\prime}=h$ (e $\mathrm{I}_{b} \circ \rho^{\prime}=\mathrm{I}_{c}$ ). Com isso, defina $\rho=\kappa \circ \rho^{\prime}$, para obtermos $f \circ \rho=f \circ \kappa \circ \rho^{\prime}=g \circ \rho^{\prime}=h$ (a igualdade $\mathrm{I}_{a} \circ \rho=\mathrm{I}_{c}$ decorre de $u$ ser terminal). A unicidade decorre facilmente do fato de $f$ ser um mono: se existe $\xi: c \rightarrow a$ tal que $f \circ \xi=h$, então $f \circ \rho=h=f \circ \xi$, e $\rho=\xi$.

$(\Leftarrow)$ se $\chi_{f}=\chi_{g}$, então $\chi_{f} \circ f=\chi_{g} \circ f=\top \circ \mathbf{I}_{a}$ e $\chi_{g} \circ g=\chi_{f} \circ g=\top \circ \mathbf{I}_{b}$. Assim, usamos os pullbacks da definição das flechas características para obter $\kappa: b \rightarrow a$ e $\rho: a \rightarrow b$ que tornam os diagramas comutativos: 

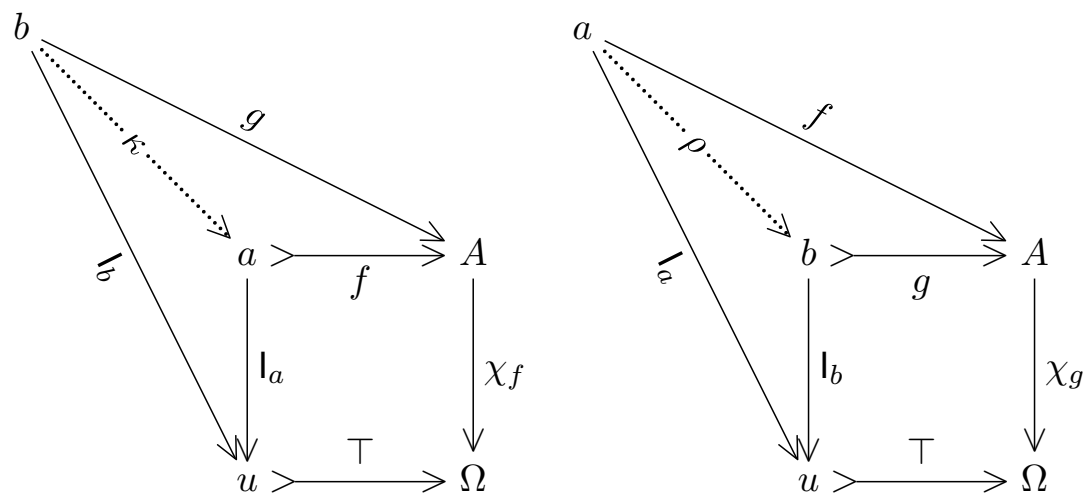

e, portanto, $[f]=[g]$.

4. Seja $f: a \longmapsto A$ em $\mathcal{C}_{1}$. Mostremos que $(a, f)$ é o equalizador de $\chi_{f}$ e $\top_{A}:=\top \circ \mathrm{I}_{A}$. Primeiramente, $\mathrm{I}_{a}=\mathrm{I}_{A} \circ f$, pois $u$ é terminal, e pela definição da flecha característica vale $\chi_{f} \circ f=\top \circ \mathbf{I}_{a}=\top \circ \mathbf{I}_{A} \circ f=\top_{A} \circ f$.

Agora, seja $g: b \rightarrow A$ tal que $\chi_{f} \circ g=\top_{A} \circ g$. Note que $\top_{A} \circ g=\top \circ \mathbf{I}_{A} \circ g=\top \circ \mathrm{I}_{b}$, novamente usando o fato de $u$ ser terminal. Assim, do pullback da definição de $\chi_{f}$, existe uma única $\kappa: b \rightarrow a$ tal que $f \circ \kappa=g$, como queríamos.

Assim, a categoria $\mathcal{C}$ ser balanceada é consequência da proposição 1.5.5.

Tendo em vista o item 3 da última proposição, dada uma categoria $\mathcal{C}$ com classificador de subobjeto $\top: \mathbf{1} \longmapsto \Omega$ e $a \in \mathcal{C}_{0}$, podemos definir um mapa tal que:

$$
[f] \mapsto \chi_{f} \in \mathcal{C}(a, \Omega), \text { para todo }[f] \in S u b(a)
$$

Por outro lado, dado $g: a \rightarrow \Omega$, podemos considerar $\check{g}: p \longmapsto a$ o pullback de $\top$ ao longo de $g$ (que será mono por ser pullback de mono). Assim, definimos um mapa:

$$
g \mapsto[\check{g}] \in \operatorname{Sub}(a), \text { para todo } g \in \mathcal{C}(a, \Omega)
$$

É fácil verificar que esses mapas são inversos (as flechas que fazem $f \sim \check{\chi_{f}}$ são obtidas dos pullbacks, e $g=\chi_{\breve{g}}$ decorre da unicidade da flecha característica), de forma que obtemos:

Lema 3.2.4. Seja $\mathcal{C}$ uma categoria localmente pequena com classificador de subobjetos $\top: u \longmapsto$ $\Omega$. Então, para todo $a \in \mathcal{C}_{0}, \mathcal{C}(a, \Omega) \cong S u b(a)$.

Em particular, vale que a categoria $\mathcal{C}$ dessa proposição é bem potenciada. Assim, a relação entre subobjetos e flechas com codomínio $\Omega$ é ainda mais forte: 
Teorema 3.2.5. Seja $\mathcal{C}$ uma categoria localmente pequena com classificador de subobjeto $\top$ : $\mathbf{1} \longmapsto \Omega$. Então, existe um isomorfismo natural $\eta: S u b(-) \Rightarrow \mathcal{C}(-, \Omega)$.

Demonstração. Pelo lema anterior, já temos os isomorfismos $\eta_{a}: S u b(a) \rightarrow \mathcal{C}(a, \Omega)$, para todo $a \in \mathcal{C}_{0}$, basta tomar $\eta_{a}([f])=\chi_{f}$. Falta a naturalidade: para toda $f: b \rightarrow a$ em $\mathcal{C}_{1}$, queremos mostrar que $\eta_{b} \circ S u b(f)=\mathcal{C}(f, \Omega) \circ \eta_{a}$. Isto é, abrindo as definições, devemos mostrar que $\chi_{m} \circ m=\chi_{m^{\prime}}\left(\right.$ com $m^{\prime}$ o pullback de $m$ ao longo de $f$ ), para toda $m: c \longmapsto a$ em $\mathcal{C}_{1}$. Para tanto, juntemos os diagramas de pullback que definem $\chi_{m}$ e $m^{\prime}$ :

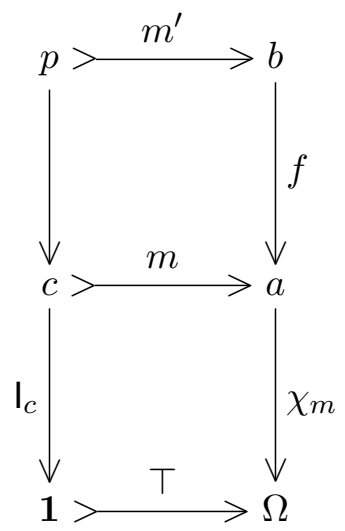

Pelo lema do pullback, o retângulo também será um pullback, e pela unicidade de flecha característica, $\chi_{m} \circ m=\chi_{m^{\prime}}$.

Aliás, a recíproca desse teorema também é verdadeira; porém, como não será usada no presente trabalho, apenas recomendamos consultar uma referência para tal resultado (por exemplo a proposição I.3.1 de [LM94]).

\subsection{Topos elementar}

Definição 3.3.1. Um topos (elementar) é uma categoria $\mathcal{E}$ cartesianamente fechada que possui limites finitos e classificador de subobjeto.

A definição original de topos elementar (de Lawvere e Tierney) também exigia que $\mathcal{E}$ possuísse colimites finitos, porém mais tarde mostrou-se que essa condição não era necessária. Existe também outra forma de definir um topos elementar, usando um objeto chamado objeto partes.

Definição 3.3.2. Seja $\mathcal{C}$ uma categoria com limites finitos. Um objeto partes de $b \in \mathcal{C}_{0}$ é um objeto $\mathcal{P}(b)$ com um mono $E_{b}: \epsilon_{b} \longmapsto \mathcal{P}(b) \times b$ universal, isto é, para todo mono $m: c \rightarrow a \times b$, 
existe um único $\hat{m}: a \rightarrow \mathcal{P}(b)$ tal que o diagrama abaixo é um quadrado de pullback:

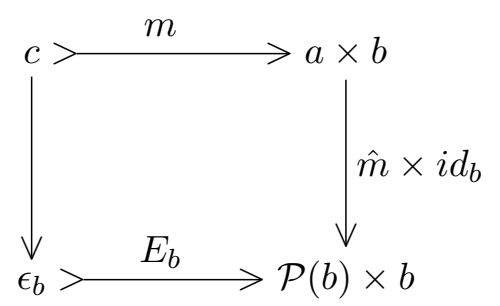

Observe que, chamando o objeto terminal de $\mathbf{1}$, o objeto partes de $\mathbf{1}$ é um classificador de subobjeto (isso é imediato considerando que $d \times \mathbf{1} \cong d$, para todo $d \in \mathcal{C}_{0}$ ). Além disso, o objeto partes e seu mono correspondente são únicos a menos de isomorfismo.

Exemplo 3.3.3. Como deve ser novamente esperado por conta da notação, em Set o objeto partes corresponde exatamente ao conjunto partes com a inclusão $E_{A}: \epsilon_{A}=\{(S, a) \in \mathcal{P}(A) \times A \mid$ $a \in S\} \hookrightarrow \mathcal{P}(A) \times A$. De fato, para todo mono $f: C^{\prime} \times A^{\prime} \hookrightarrow C \times A$, defina $\hat{f}: C \rightarrow \mathcal{P}(A)$ tomando, para todo $c \in C, \hat{f}(c)=\left\{a \in A \mid(c, a) \in C^{\prime} \times A^{\prime}\right\}$. Dessa forma, lembrando a forma dos pullbacks em Set (exemplo 1.4.9), o (objeto do) pullback $P$ de $\hat{f} \times i d_{a}$ e $E_{a}$ será:

$$
\begin{aligned}
P & =\left\{((S, a),(c, a)) \in \epsilon_{A} \times(C \times A) \mid(\hat{f}(c), a)=E_{A}(S, a)\right\} \\
& =\left\{((S, a),(c, a)) \in \epsilon_{A} \times(C \times A) \mid(\hat{f}(c), a) \in \epsilon_{A}\right\} \\
& =\left\{((S, a),(c, a)) \in \epsilon_{A} \times(C \times A) \mid a \in \hat{f}(c)\right\} \\
& =\left\{((S, a),(c, a)) \in \epsilon_{A} \times(C \times A) \mid(c, a) \in C^{\prime} \times A^{\prime}\right\}
\end{aligned}
$$

Assim, podemos definir funções inversas entre $P$ e $C^{\prime} \times A^{\prime}$ fazendo:

$$
((S, a),(c, a)) \mapsto(c, a) \quad \text { e } \quad(c, a) \mapsto((\hat{f}(c), a),(c, a))
$$

de forma que $C^{\prime} \times A^{\prime}$ é isomorfo ao pullback. Finalmente, para a unicidade, seja $g: C \rightarrow \mathcal{P}(A)$ tornando o diagrama um pullback. Então, como nas igualdades de $P$ acima, temos:

$$
\begin{aligned}
P=\left\{((S, a),(c, a)) \in \epsilon_{A} \times(C \times A) \mid(c, a) \in C^{\prime} \times A^{\prime}\right\}= \\
=\left\{((S, a),(c, a)) \in \epsilon_{A} \times(C \times A) \mid a \in g(c)\right\}
\end{aligned}
$$

Ou seja, para todos $a \in A, c \in C, a \in g(c)$ se, e somente se, $(c, a) \in C^{\prime} \times A^{\prime}$, que é exatamente a definição de $\hat{f}(c)$. Logo, $\hat{f}=g$, como queríamos.

Teorema 3.3.4. Seja $\mathcal{E}$ uma categoria com limites finitos. Então, são equivalentes: 
1. $\mathcal{E}$ é um topos (elementar);

2. $\mathcal{E}$ possui objeto partes.

Demonstração. Em relação à implicação $(2 \Rightarrow 1)$, apesar de ser fácil definir o classificador de subobjeto (como feito na observação acima), mostrar que a categoria é cartesianamente fechada requer um detalhamento técnico que pouco contribuiria ao presente trabalho. Assim, façamos aqui apenas a implicação $(1 \Rightarrow 2)$; para a recíproca, recomenda-se consultar a seção 5.4 de [BW85], ou a seção A2.3 de [Joh02a].

$(1 \Rightarrow 2)$ Seja $\top: \mathbf{1} \rightarrow \Omega$ o classificador de subobjeto de $\mathcal{E}$. Para cada $b \in \mathcal{C}_{0}$, tome $\mathcal{P}(b)=\Omega^{b}$ e $E_{b}: \epsilon_{b} \longmapsto \Omega^{b} \times b$ como o pullback de $\top$ ao longo de $e v_{\Omega}^{b}$ (logo um mono, pois $\top$ é mono). Para mostrar que isso define um objeto partes, seja $m: c \longmapsto a \times b$ um mono, e defina $\hat{m}=\exp \left(\chi_{m}\right)$, a transposta exponencial da flecha característica de $m$, como nos diagramas:
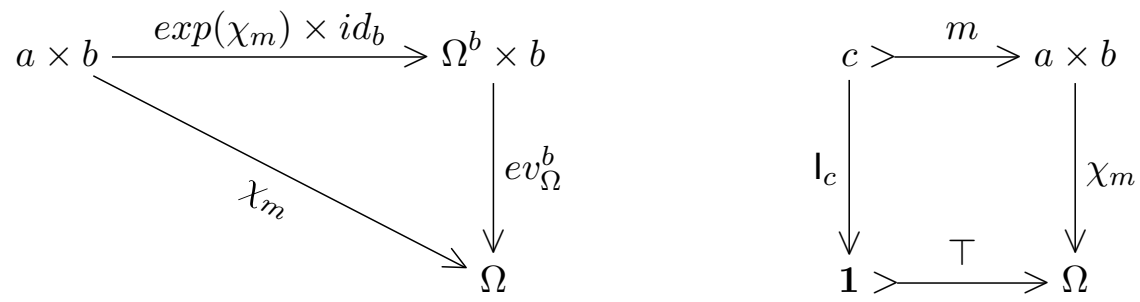

Assim, temos a igualdade:

$$
e v_{\Omega}^{b} \circ\left(\exp \left(\chi_{m}\right) \times i d_{b}\right) \circ m=\chi_{m} \circ m=\top \circ \mathbf{I}_{c}
$$

e, usando a definição de $E_{b}$ pelo pullback, existe uma única $\kappa: c \rightarrow \epsilon_{b}$ tornando o diagrama abaixo comutativo:

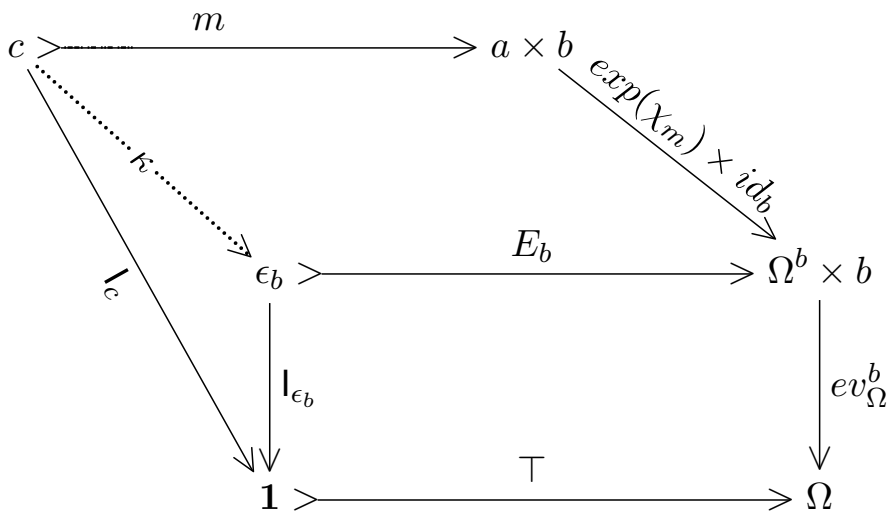

Como os diagramas com vértices $\epsilon_{b}, \Omega^{b} \times b, \Omega, \mathbf{1}$ (retângulo inferior) e $c, a \times b, \Omega, \mathbf{1}$ (fronteira do 
diagrama acima sem $I_{c}$ ) são pullbacks, pelo lema do pullback o diagrama:

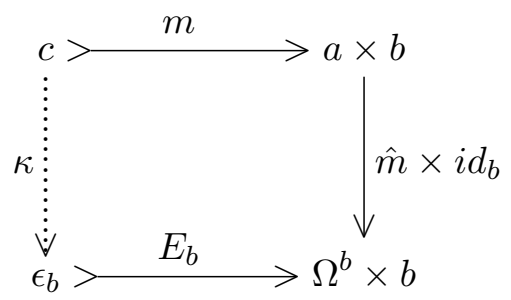

também é um pullback. Agora a unicidade: seja $n: a \rightarrow \Omega^{b}$ tal que o quadrado superior no diagrama abaixo é um pullback:

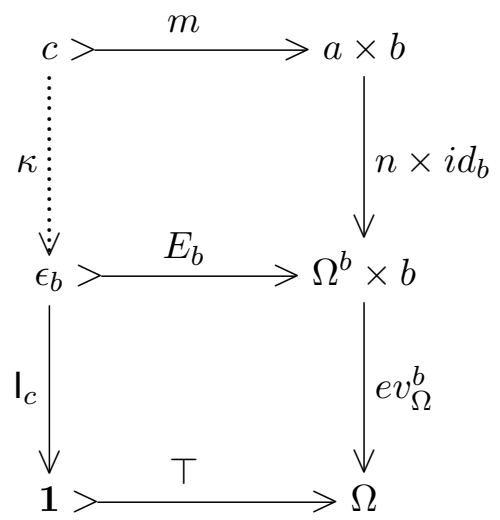

Como o quadrado inferior também é um pullback, novamente usamos o lema do pullback e obtemos que

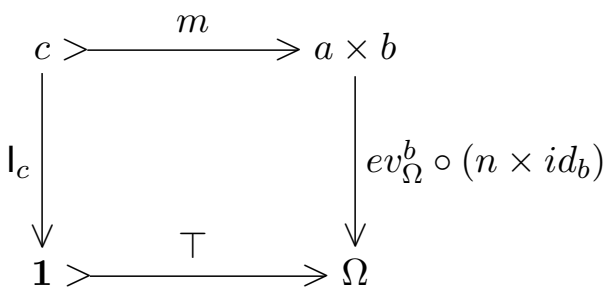

é um pullback. Portanto, pela unicidade da flecha característica, $e v_{\Omega}^{b} \circ\left(n \times i d_{b}\right)=\chi_{m}$, e pela unicidade da transposta exponencial, $n=\exp \left(\chi_{m}\right)$, como queríamos.

Para topoi elementares, temos duas noções de morfismos, o que reflete o fato da teoria de topos ter influências tanto da lógica quanto da geometria. O primeiro dos morfismos é definido de forma a preservar a estrutura da definição de topos elementar:

Definição 3.3.5. Sejam $\mathcal{E}$ e $\mathcal{E}^{\prime}$ topoi, com objetos partes $\mathcal{P}$ e $\mathcal{P}^{\prime}$ e monos correspondentes $E_{(-)}$ e $E_{(-)}^{\prime}$, respectivamente. Um funtor lógico $F: \mathcal{E} \rightarrow \mathcal{E}^{\prime}$ é um funtor que preserva limites finitos e objeto partes, isto é, para todo $a \in \mathcal{E}_{0}$, existe um iso de flechas entre $F_{1}\left(E_{a}\right)$ e $E_{F_{0}(a)}^{\prime}(\mathrm{em}$ particular, $\left.F_{0}(\mathcal{P}(a)) \cong \mathcal{P}^{\prime}\left(F_{0}(a)\right)\right)$. 
Proposição 3.3.6. Sejam $\mathcal{E}$ e $\mathcal{E}^{\prime}$ topoi e $F: \mathcal{E} \rightarrow \mathcal{E}^{\prime}$ um funtor que preserva limites finitos. Então, são equivalentes:

1. F preserva classificador de subobjeto e exponenciação;

2. F é um funtor lógico.

Essa proposição usa as construções da demonstração do teorema anterior: a implicação (1 $\Rightarrow 2$ ) pode ser indicada facilmente:

$$
F_{0}(\mathcal{P}(a))=F_{0}\left(\Omega^{a}\right) \cong F_{0}(\Omega)^{F_{0}(a)} \cong \Omega^{\prime F_{0}(a)}=\mathcal{P}^{\prime}\left(F_{0}(a)\right)
$$

tal como a parte da recíproca que se refere ao classificador de subobjeto:

$$
F_{0}(\Omega) \cong F_{0}(\mathcal{P}(\mathbf{1})) \cong \mathcal{P}^{\prime}\left(F_{0}(\mathbf{1})\right) \cong \mathcal{P}^{\prime}\left(\mathbf{1}^{\prime}\right) \cong \Omega^{\prime}
$$

O fato de funtores lógicos preservarem exponenciação está relacionado com o trecho que não fizemos da demonstração do teorema acima. Esse resultado é mostrado na proposição A2.3.7 de [Joh02a].

No último capítulo, definimos morfismos geométricos entre topoi de Grothendieck, que usava apenas adjunção e preservação de limites. Assim, podemos definir morfismos geométricos para topoi elementares também:

Definição 3.3.7. Sejam $\mathcal{E}, \mathcal{E}^{\prime}$ topoi (elementares). Um morfismo geométrico é um par de funtores $\left(\varphi_{*}, \varphi^{*}\right): \mathcal{E} \rightarrow \mathcal{E}^{\prime}, \operatorname{com} \varphi_{*}: \mathcal{E} \rightarrow \mathcal{E}^{\prime}$ e $\varphi^{*}: \mathcal{E}^{\prime} \rightarrow \mathcal{E}$, tal que $\varphi^{*} \dashv \varphi_{*}$ e $\varphi^{*}$ preserva limites finitos.

\subsection{Algumas propriedades de topoi}

Teorema 3.4.1. Todo topos é finitamente cocompleto.

A forma mais comum de provar isso usa que o objeto partes define um funtor $\mathcal{P}: \mathcal{E}^{o p} \rightarrow \mathcal{E}$, e que esse funtor é monádico. Mônadas estão um pouco fora do escopo do presente trabalho, mas façamos um breve resumo da ideia do argumento.

Primeiramente, para definir o funtor $\mathcal{P}: \mathcal{E}^{o p} \rightarrow \mathcal{E}$, dada $f: a \rightarrow b$ em $\mathcal{E}_{1}$, considere o mono 
$m_{f}: E_{f} \longmapsto \mathcal{P}(b) \times a$ obtido pelo pullback:

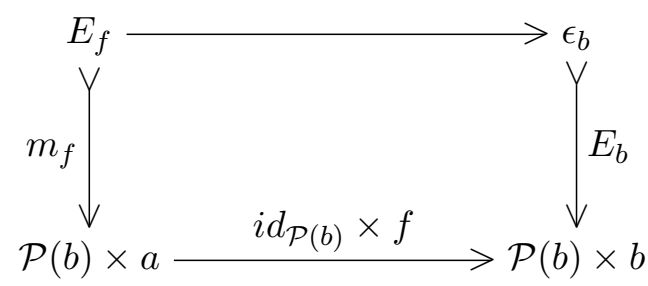

Assim, definimos

$$
\begin{gathered}
\mathcal{P}_{0}(a):=\mathcal{P}(a), \text { para todo } a \in \mathcal{E}_{0} \\
\mathcal{P}_{1}(f): \mathcal{P}(b) \rightarrow \mathcal{P}(a) \text {, para toda } f: a \rightarrow b \text { em } \mathcal{E}_{1} \text {, onde } \mathcal{P}_{1}(f):=\hat{m}_{f}
\end{gathered}
$$

com $\hat{m}_{f}$ a flecha única usando o mono $m_{f}$ acima na definição de objeto partes.

Agora, uma mônada numa categoria $\mathcal{C}$ é uma tripla $\mathbb{T}=(T, \eta, \theta)$, com $T: \mathcal{C} \rightarrow \mathcal{C}$ um funtor e $\eta: i d_{\mathcal{C}} \Rightarrow T$ e $\mu: T \circ T \Rightarrow T$ transformações naturais, tal que os diagramas abaixo são comutativos:
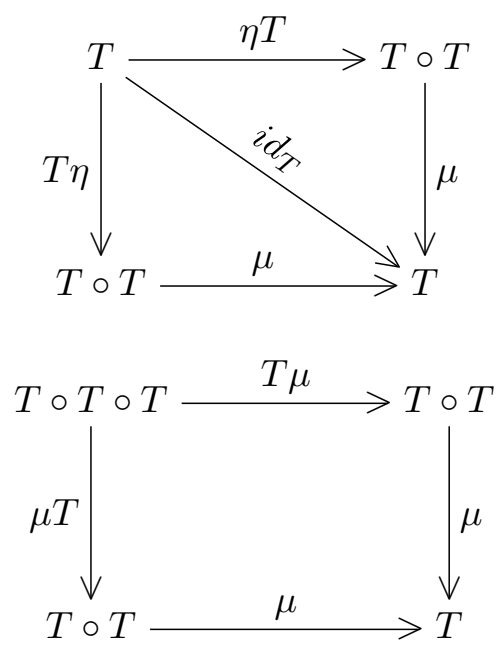

Pensando em $\mu$ como a operação de um monoide, o primeiro diagrama representa a existência da identidade (dada por $\eta$ ) para essa operação, e o segundo representa a associatividade.

A importância das mônadas para o estudo de topos reside em como elas se relacionam com funtores adjuntos. Toda adjunção $L \dashv R$ dá origem a uma mônada $(R \circ L, \eta, R \theta L)$, com $\eta$ a unidade e $\theta$ a counidade da adjunção. Aliás, toda mônada pode ser obtida de uma adjunção (geralmente não única); uma das construções dessa adjunção usa a categoria das álgebras $\mathcal{C}^{\mathbb{T}}$ e o funtor esquecimento $U_{\mathbb{T}}: \mathcal{C}^{\mathbb{T}} \rightarrow \mathcal{C}$. Essa categoria possui algumas características interessantes, dentre elas o fato de que se $\mathcal{C}$ possui um certo tipo de limite, então $\mathcal{C}^{\mathbb{T}}$ também o possui, e esse limite é preservado por $U_{\mathbb{T}}$. 
Finalmente, um funtor $F: \mathcal{A} \rightarrow \mathcal{B}$ é dito monádico se existem uma mônada $\mathbb{T}$ em $\mathcal{B}$ e uma equivalência de categorias $G: \mathcal{A} \rightarrow \mathcal{B}^{\mathbb{T}}$ tais que $U_{\mathbb{T}} \circ G$ é isomorfo a $F$. Assim, em particular, se $\mathcal{B}$ é completa, a equivalência $G$ garante que $\mathcal{A}$ também o seja. Para o caso específico desse teorema, dado um topos $\mathcal{E}$, se o funtor partes $\mathcal{P}: \mathcal{E}^{o p} \rightarrow \mathcal{E}$ é monádico, então, como todo topos é finitamente completo por definição, $\mathcal{E}^{o p}$ também é uma categoria finitamente completa; isto é, $\mathcal{E}$ é finitamente cocompleto.

Para uma apresentação mais completa da teoria de mônadas, pode-se consultar o capítulo 4 de [Bor08b], ou os capítulos 4 e 9 de [BW85] (sendo o capítulo 4 deste último dedicado mais especificamente aos aspectos da teoria usados na teoria de topos). Já a construção de que o funtor partes é monádico é feita com detalhes na seção 5.1 de [BW85], na seção 5.7 de [Bor08c] e na seção A2.2 de [Joh02a].

Teorema 3.4.2. Teorema fundamental da teoria de topos. Sejam $\mathcal{E}$ um topos e a $\in \mathcal{E}_{0}$. Então, a categoria slice $\mathcal{E} \downarrow$ a também é um topos.

Como em resultados anteriores, mostrar que a categoria slice é cartesianamente fechada é bastante longo, então façamos aqui apenas o classificador de subobjeto (o fato de possuir limites finitos é a proposição 1.8.5). A demonstração completa, porém, pode ser encontrada em diversas referências, dentre as quais citamos: o início da seção A2.3 de [Joh02a], o teorema IV.7.1 de [LM94], ou o teorema 17.4 de [Mc195].

Demonstração. Seja $T: \mathbf{1} \longmapsto \Omega$ o classificador de subobjeto de $\mathcal{E}$. Mostremos que a a flecha $\left\langle i d_{a}, \top_{a}\right\rangle:\left(a, i d_{a}\right) \rightarrow\left(a \times \Omega, \pi_{1}\right)$ é o classificador de subobjetos de $\mathcal{E} \downarrow a$, onde $\pi_{1}: a \times \Omega \rightarrow a$ é a projeção na primeira coordenada.

Primeiramente, observe que se uma flecha $h:(b, f) \rightarrow(c, g)$ é um mono em $\mathcal{E} \downarrow a$, então para todas flechas tais que o diagrama abaixo comuta:

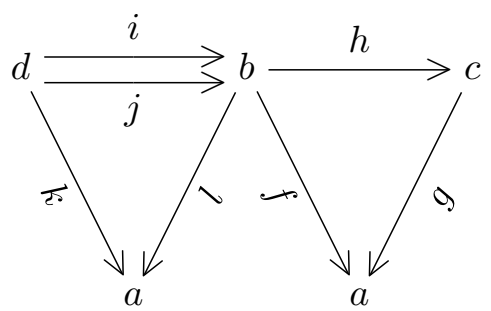

temos $i=j$. Logo, $h$ é um mono em $\mathcal{E}$.

Com isso, nosso objetivo será mostrar que, para qualquer mono $h:(b, f) \rightarrow(c, g)$ o seguinte 
diagrama em $\mathcal{E} \downarrow a$ é um pullback:

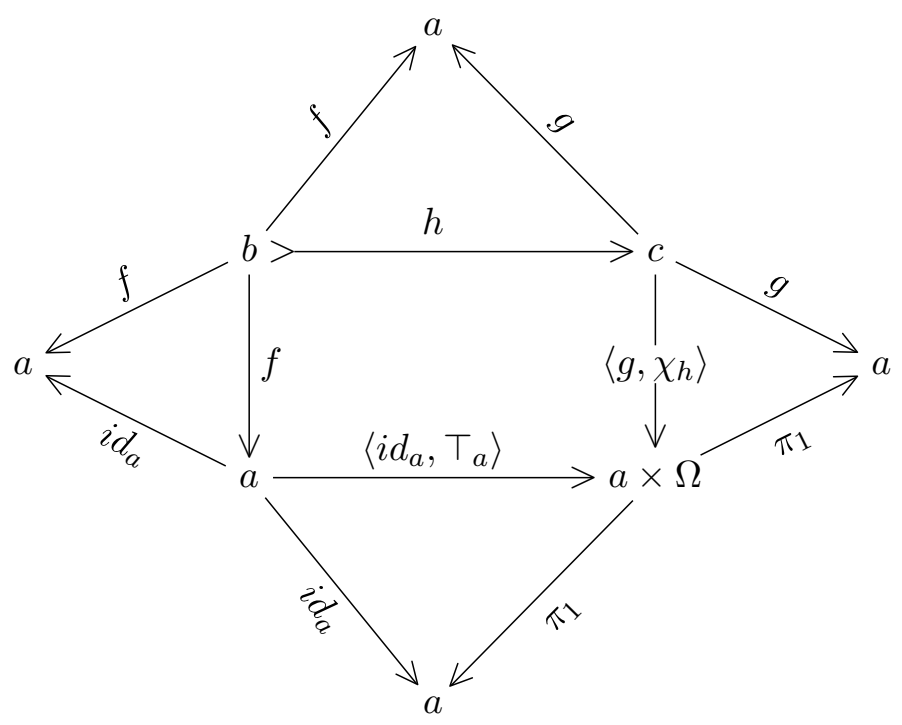

Note que esse diagrama, de fato, comuta, pois $g \circ h=i d_{a} \circ f=f$ pela definição de $h$, e pela definição de $\chi_{h}$ temos:

$$
\chi_{h} \circ h=\top \circ \mathrm{I}_{b}=\top \circ \mathrm{I}_{a} \circ f=\top_{a} \circ f
$$

(usamos aqui, também, a unicidade de $\mathrm{I}_{b}: b \rightarrow \mathbf{1}$ ).

Agora, considere flechas: $\alpha:(d, \alpha) \rightarrow\left(a, i d_{a}\right), \gamma:(d, \alpha) \rightarrow(c, g)$ tais que $\left\langle g, \chi_{h}\right\rangle \circ \gamma=$ $\left\langle i d_{a}, \top_{a}\right\rangle \circ \alpha$. Isso implica em (usando argumentação semelhante à acima):

$$
\chi_{h} \circ \gamma=\top_{a} \circ \alpha=\top \circ \mathbf{I}_{a} \circ \alpha=\top \circ \mathbf{I}_{d}
$$

o que nos permite usar o pullback da definição de $\chi_{h}$ para obter a única $\beta: d \rightarrow b$ que torna o diagrama abaixo comutativo:

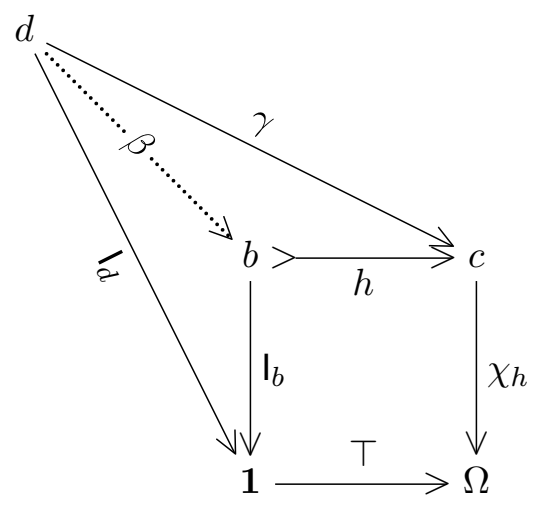

Note que $h \circ \beta=\gamma$ implica em $g \circ h \circ \beta=g \circ \gamma$, logo $f \circ \beta=\alpha$ (por causa das definições das flechas $h$ e $\gamma$ ). Portanto, $\beta$ é uma flecha em $\mathcal{E} \downarrow a$ e também serve para o diagrama (1) (na 
categoria comma):

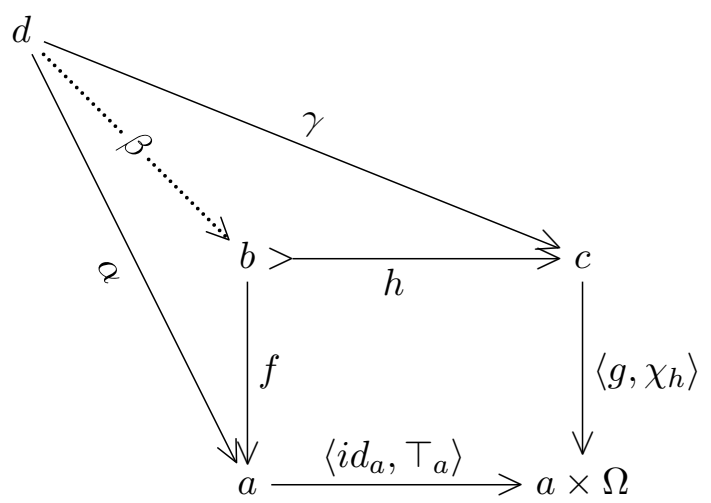

A unicidade de $\beta$ decorre facilmente de $h$ ser mono; explicitamente, seja $k$ que também torna o diagrama acima comutativo, então $h \circ \kappa=\gamma=h \circ \beta$, donde $\kappa=\beta$. Logo, o diagrama é um pullback.

Falta apenas mostrar a unicidade de $\left\langle g, \chi_{h}\right\rangle$. Suponha que a flecha $\langle\mu, \lambda\rangle:(c, g) \rightarrow\left(a \times \Omega, \pi_{1}\right)$ também torne o diagrama um pullback. De imediato da projeção, já temos $\mu=g$. Agora, ser pullback garante que o diagrama

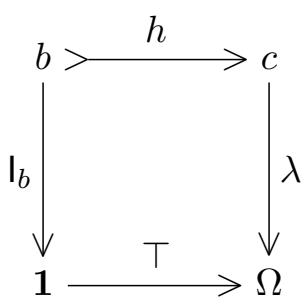

também seja um pullback (novamente fazendo $\lambda \circ h=\top_{a} \circ f=\top \circ \mathbf{I}_{b}$ ), e a unicidade na definição da flecha característica nos fornece $\chi_{h}=\lambda$.

Proposição 3.4.3. Sejam $\mathcal{E}, \mathcal{E}^{\prime}$ topoi e $F: \mathcal{E} \rightarrow \mathcal{E}^{\prime}$ um funtor lógico. Então:

\section{F preserva colimites;}

2. F possui adjunto à esquerda se, e somente se, $F$ possui adjunto à direita.

Ambos os itens usam que os funtores partes no diagrama abaixo são monádicos:

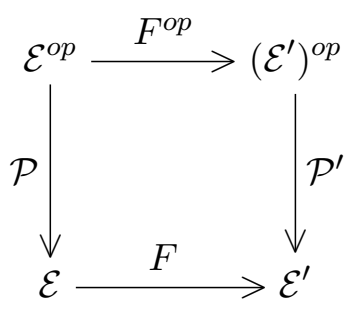


Ver, por exemplo, a proposição 5.3.4 de [BW85] ou a proposição 5.11.3 de [Bor08c] para o item (1), e a proposição 5.3.5 de [BW85] para o item (2)

Proposição 3.4.4. Sejam $\mathcal{E}$ um topos e $a \in \mathcal{E}_{0}$. Considere o funtor $a^{\star}: \mathcal{E} \rightarrow \mathcal{E} \downarrow$ a (como definido em 1.8.7). Então:

1. $a^{\star}$ é um funtor lógico;

2. $a^{\star}$ possui adjuntos $\Sigma_{a} \dashv a^{\star} \dashv \Pi_{a}$.

Demonstração. 1. A preservação do classificador de subobjeto é imediata do fato de que o classificador em $\mathcal{E} \downarrow a$ é $\left\langle i d_{a}, \top_{a}\right\rangle:\left(a, i d_{a}\right) \rightarrow\left(a \times \Omega, \pi_{1}\right)$. Para a exponenciação, precisamos provar que, para todos $c, d \in \mathcal{E}_{0}, a_{0}^{*}\left(d^{c}\right)=\left(a \times d^{c}, \pi_{1}\right)$ corresponde à $c_{0}^{*}(d)^{c_{0}^{*}(c)}$; chamemos $c_{0}^{*}(c)=\left(a \times c, \pi_{1}^{c}\right)$ e $c_{0}^{*}(d)=\left(a \times d, \pi_{1}^{d}\right)$. Note que, para toda $f: b \rightarrow a$ em $\mathcal{E}_{1}$, o diagrama abaixo é um pullback:

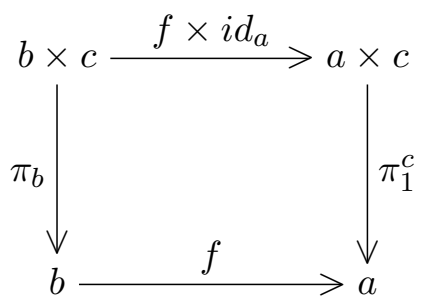

(para quaisquer $\gamma_{1}, \gamma_{2}$ tais que $f \circ \gamma_{1}=\pi_{1}^{c} \circ \gamma_{2}$, basta tomar a flecha única como $\left\langle\gamma_{1}, \pi_{2}^{c} \circ \gamma_{2}\right\rangle$ ). Como vimos na seção de categorias slice, produtos na categoria slice são os pullbacks, logo o diagrama acima nos fornece o produto de $f$ por $\pi_{1}^{c}$ em $\mathcal{E} \downarrow a$. Ou seja, dar uma flecha $(b, f) \times\left(a \times c, \pi_{1}^{c}\right) \rightarrow\left(a \times d, \pi_{1}^{d}\right)$ corresponde a dar uma flecha $\alpha:\left(b \times c, f \circ \pi_{b}\right) \rightarrow\left(a \times d, \pi_{1}\right) ;$ que por sua vez corresponde a uma flecha $b \times c \rightarrow d$ (isto porque o "valor de $\alpha$ na primeira coordenada" já está determinado pelo fato de $\left.\pi_{1} \circ \alpha=f \circ \pi_{b}\right)$.

Assim, é fácil obter as correspondências:

$$
\mathcal{E} \downarrow a\left((b, f),\left(a \times d^{c}, \pi_{1}\right)\right) \cong \mathcal{E}\left(b, d^{c}\right) \cong \mathcal{E}(b \times c, d) \cong \mathcal{E} \downarrow a\left((b, f) \times\left(a \times c, \pi_{1}^{c}\right),\left(a \times d, \pi_{1}^{d}\right)\right)
$$

e, usando que $\mathcal{E} \downarrow a$ é cartesianamente fechada, concluímos que $a_{0}^{*}\left(d^{c}\right)=c_{0}^{*}(d)^{c_{0}^{*}(c)}$.

2. Já mostramos que $a^{*}$ possui adjunto à esquerda no teorema 1.8.8, e pela proposição anterior, $a^{*}$ também possui adjunto à direita. 
Como consequência disso, e usando que um slice de um topos também é topos, obtemos resultado semelhante para o funtor $f^{\star}$.

Proposição 3.4.5. Sejam $\mathcal{E}$ um topos e $f: a \rightarrow b$ em $\mathcal{E}_{1}$. Considere o funtor $f^{\star}: \mathcal{E} \downarrow b \rightarrow \mathcal{E} \downarrow a$ (como definido em 1.8.9). Então:

1. $f^{\star}$ é um funtor lógico;

2. $f^{\star}$ possui adjuntos $\Sigma_{f} \dashv f^{\star} \dashv \Pi_{f}$.

Corolário 3.4.6. Pullbacks de epis são epis num topos.

Demonstração. Sejam $\mathcal{E}$ um topos e $f: a \rightarrow b$ em $\mathcal{E}_{1}$. Então, para todos $h, h^{\prime}: b \rightarrow b^{\prime}$ em $\mathcal{E}$, $h \circ f=h^{\prime} \circ f$ implica $h=h^{\prime}$, ou $h \circ i d_{b}=h=h^{\prime}=h^{\prime} \circ i d_{b}$. Assim, o diagrama

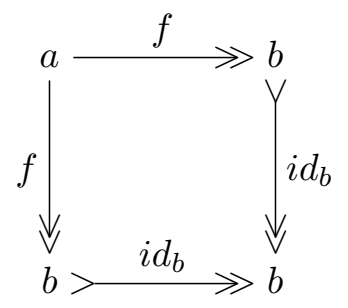

é um pushout em $\mathcal{E}$. Dessa forma, é fácil verificar que seguinte diagrama também é um pushout em $\mathcal{E} \downarrow b$ :

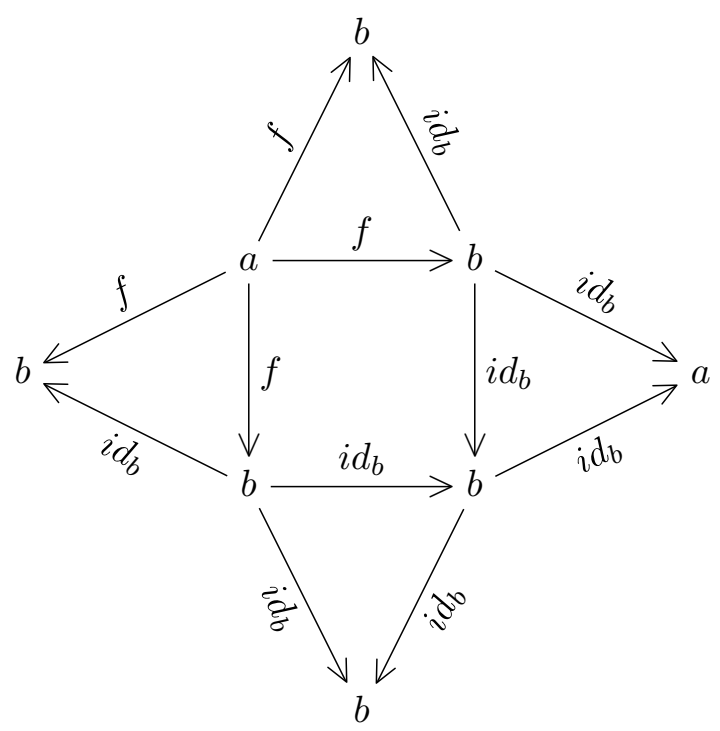

Agora, para qualquer flecha $g: c \rightarrow b$ em $\mathcal{E}_{1}$, o funtor $g^{\star}: \mathcal{E} \downarrow b \rightarrow \mathcal{E} \downarrow c$ preserva colimites. Logo, aplicando $g^{\star}$ ao diagrama acima, obtemos um pushout em $\mathcal{E} \downarrow c$, e, como o funtor esquecimento $\Sigma_{c}: \mathcal{E} \downarrow c \rightarrow \mathcal{E}$ preserva colimites (pois possui adjunto à direita, ver teorema 1.7.5), concluímos 
que o seguinte diagrama em $\mathcal{E}$

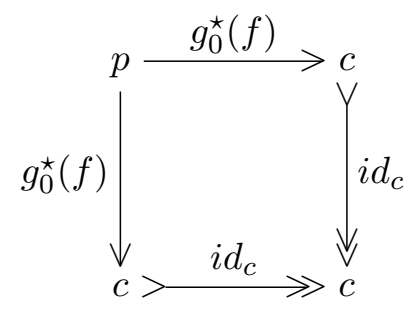

também é um pushout. Com isso, sejam $k, k^{\prime}: c \rightarrow c^{\prime}$ tais que $k \circ g_{0}^{\star}(f)=k^{\prime} \circ g_{0}^{\star}(f)$. Então, existe uma única flecha $\kappa: c \rightarrow c^{\prime}$ tal que $\kappa \circ i d_{c}=k$ e $\kappa \circ i d_{c}=k^{\prime}$, ou seja, $k=k^{\prime}$. Logo, o pullback de $f$ ao longo de $g$ é um epi.

Corolário 3.4.7. Coprodutos são estáveis sob pullbacks num topos.

O significado preciso desse enunciado ficará claro na demonstração:

Demonstração. Sejam $\mathcal{E}$ um topos e $f: a \rightarrow b$ em $\mathcal{E}_{1}$. Para cada $i \in I$, sejam $\alpha_{i}: a_{i} \rightarrow a$ e $\beta_{i}: b_{i} \rightarrow b$ flechas em $\mathcal{E}_{1}$ tais que $\alpha_{i}$ é o pullback de $\beta_{i}$ ao longo de $f$. Consideremos o coproduto $\left(\coprod_{i \in I} b_{i},\left(\iota_{i}\right)_{i \in I}\right)$ e a flecha única $\kappa: \coprod_{i \in I} b_{i} \rightarrow b$ tal que $\kappa \circ \iota_{i}=\beta_{i}$, para todo $i \in I$. Então, façamos o pullback de $\kappa$ e $f$ :

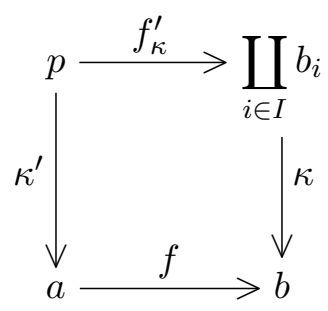

Agora, chamando de $\left(a_{i}, f_{i}^{\prime}, \alpha_{i}\right)$ o pullback de $f$ e $\beta_{i}$ (para cada $i \in I$ ), temos $f \circ \alpha_{i}=\beta_{i} \circ f_{i}^{\prime}=$ $\kappa \circ \iota_{i} \circ f_{i}^{\prime}$. Logo, usando que o diagrama acima é um pullback, para cada $i \in I$ existe uma flecha única $\kappa_{i}: a_{i} \rightarrow p$ tal que $f_{\kappa}^{\prime} \circ \kappa_{i}=\iota_{i} \circ f_{i}^{\prime}\left(\mathrm{e} \kappa^{\prime} \circ \kappa=\alpha_{i}\right)$. Assim, para todo $i \in I$ o quadrado superior do diagrama abaixo é comutativo:

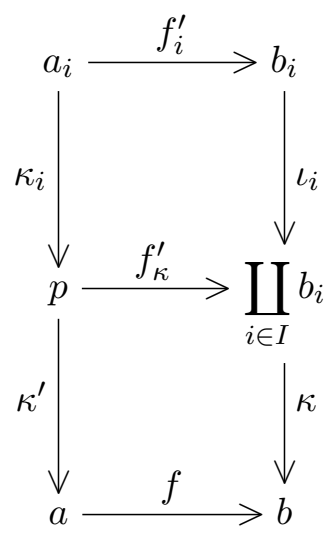


O retângulo e o quadrado inferior são pullbacks por definição, portanto o quadrado superior também é um pullback, ou seja, cada $\kappa_{i}$ é o pullback de $\iota_{i}$ ao longo de $f_{\kappa}^{\prime}$. Finalmente, pois, usando que $f^{\star}$ preserva colimites, $\left(p,\left(\kappa_{i}\right)_{i \in I}\right)$ também será um coproduto, de forma que $p \cong \coprod_{i \in I} a_{i}$ e temos o diagrama de pullback:

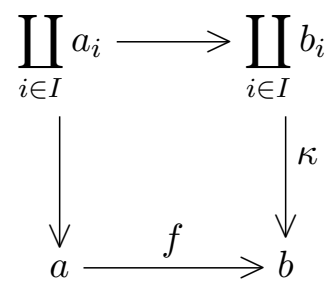

Teorema 3.4.8. Toda flecha num topos possui fatoração epi-mono.

Demonstração. Sejam $\mathcal{E}$ um topos e $f: a \rightarrow b$ em $\mathcal{E}_{1}$. Considere $\left(q, i_{1}, i_{2}\right)$ o cokernel par de $f$. Defina a flecha $\operatorname{im}(f): f(a) \rightarrow b$ como (a flecha do) equalizador de $i_{1}$ e $i_{2}$ (logo, um mono por 1.5.3). Como $i_{1} \circ f=i_{2} \circ f$, existe uma única $f^{\triangleright}: a \rightarrow f(a)$ tal que o diagrama abaixo comuta:

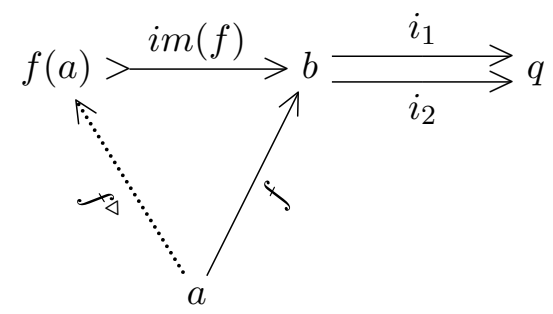

Agora, sejam $g: c \rightarrow b$ mono e $h: a \rightarrow c$ qualquer em $\mathcal{E}_{1}$ tais que $f=g \circ h$. Como monos em $\mathcal{E}$ são regulares (proposição 3.2.3), $(c, g)$ é o equalizador de um par de flechas $\delta_{1}, \delta_{2}: b \rightarrow d$ em $\mathcal{E}_{1}$, donde $\delta_{1} \circ f=\delta_{1} \circ g \circ h=\delta_{2} \circ g \circ h=\delta_{2} \circ f$. Assim, pelo pushout existe um único $\kappa: q \rightarrow d$ tornando o diagrama abaixo comutativo:

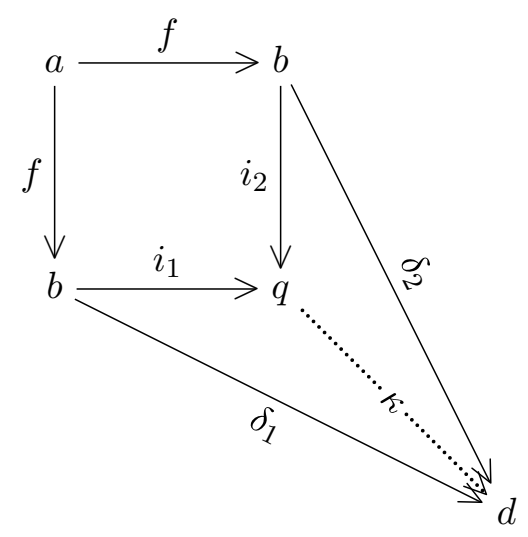

Com isso, e usando a definição de $i m(f)$ como o equalizador, temos $\delta_{1} \circ i m(f)=\kappa \circ i_{1} \circ i m(f)=$ 
$\kappa \circ i_{2} \circ i m(f)=\delta_{2} \circ i m(f)$. Usando agora que $g$ é equalizador, temos que existe uma única $\rho: f(a) \rightarrow c$ tal que o diagrama abaixo é comutativo:

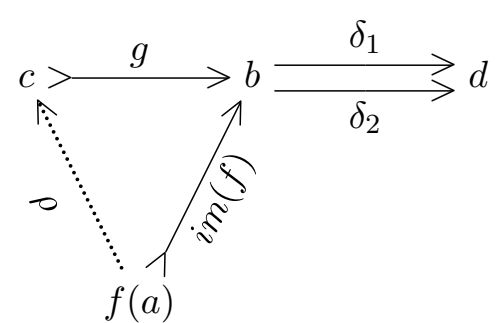

e $g \circ \rho=i m(f)$. Em outras palavras, para todo mono $g$ tal que existe $h$ com $f=g \circ h$, vale $[i m(f)] \leq[g]$. Esse fato, que pode ser compreendido como $[i m(f)]$ ser o "menor subobjeto de $b$ pelo qual $f$ se fatora", será útil em outras demonstrações mais adiante.

Além disso, $g \circ \kappa \circ f^{\triangleright}=i m(f) \circ f^{\triangleright}=f=g \circ h$, e como $g$ é mono, chegamos a $\kappa \circ f^{\triangleright}=h$, e temos o diagrama comutativo:

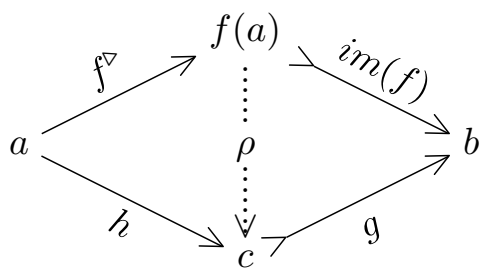

Agora, decompondo $f^{\triangleright}$ com a imagem como fizemos para $f$ acima, temos $f=i m(f) \circ f^{\triangleright}=$

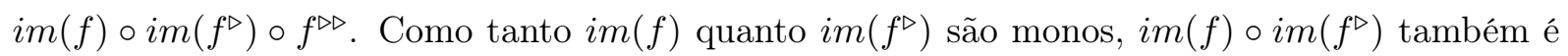
mono, e (como vimos acima) $[i m(f)] \leq\left[i m(f) \circ i m\left(f^{\triangleright}\right)\right]$.

Por outro lado, é imediato da definição que $\left[i m(f) \circ i m\left(f^{\triangleright}\right)\right] \leq[i m(f)]$. Logo, $[i m(f)]=$ $\left[i m(f) \circ i m\left(f^{\triangleright}\right)\right]$, e existe um iso $\xi: f^{\triangleright}(a) \rightarrow f(a)$ tal que $i m(f) \circ \xi=i m(f) \circ i m\left(f^{\triangleright}\right)$. Mas usando novamente que $i m(f)$ é mono, daí tiramos que $\xi=i m\left(f^{\triangleright}\right)$, e $i m\left(f^{\triangleright}\right)$ é um iso.

Dessa forma, chamando de $\left(p, j_{1}, j_{2}\right)$ o cokernel par de $f^{\triangleright}$, por definição de $i m\left(f^{\triangleright}\right)$ como equalizador temos $j_{1} \circ i m\left(f^{\triangleright}\right)=j_{2} \circ i m\left(f^{\triangleright}\right)$, $\log \circ j_{1}=j_{2}$ pois $i m\left(f^{\triangleright}\right)$ é epi (por ser um iso). Portanto, usando o dual da proposição 1.5.8, concluímos que $f^{\triangleright}$ é um epi.

Finalmente, a flecha $\rho: f(a) \rightarrow c$ é um mono pois $i m(f)$ é mono e $g \circ \rho=i m(f)$, e se $h$ for epi $\rho$ também será epi pois $\rho \circ f^{\triangleright}=h$; e como todo topos é balanceado (proposição 3.2.3 novamente), $\rho$ será um iso. Ou seja, se $f$ possui outra decomposição epi-mono $f=g \circ h$, existirá 
um único iso $\rho: f(a) \rightarrow c$ tornando o diagrama comutativo:

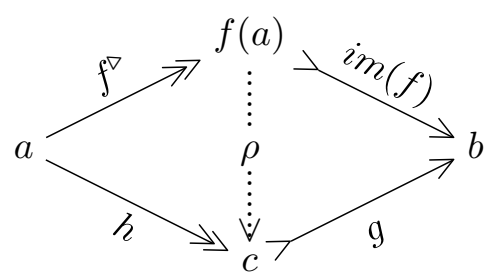

e $f$ possui fatoração epi-mono $f=i m(f) \circ f^{\triangleright}$.

Para finalizar essa seção, um resultado relacionando morfismos geométricos e funtores lógicos:

Proposição 3.4.9. Um funtor lógico $F$ é a imagem direta de um morfismo geométrico se, e somente se, F for uma equivalência de categorias.

Note que, assim, a natureza do que é preservado por funtores lógicos e morfismos geométricos é, de fato, bem distinta, ao ponto de ambas as noções coincidirem apenas no caso de uma equivalência de categorias. Para a demonstração, ver escólio A2.3.9 de [Joh02a].

\subsection{Exemplos de topoi}

Exemplo 3.5.1. O exemplo inicial de topos é, naturalmente, Set, e boa parte da estrutura de topos já foi indicada em exemplos anteriores: Set possui produtos e equalizadores, logo é completa (proposição 1.5.11) ${ }^{1}$; é cartesianamente fechada com exponenciação $b^{a}$ o conjunto das funções $a \rightarrow b$ (exemplo 3.1.2); e o classificador de subobjeto é $T:\{*\} \rightarrow\{0,1\}$, com flechas características simplesmente as funções características de conjuntos (exemplo 3.2.2).

Alguns exemplos mais elementares de topoi decorrem facilmente do fato de Set ser topos, por exemplo Set $_{f i n}$ (a categoria dos conjuntos finitos) e Set $\times$ Set.

Exemplo 3.5.2. Seja $\mathcal{C}$ uma categoria pequena. Então, Set $^{\mathcal{C}^{o p}}$, a categoria dos prefeixes, é um topos. Ser finitamente completa vem do corolário 1.5.17, usando que Set é completa.

\footnotetext{
${ }^{1}$ Também é possível construir os limites em Set de forma explícita: para $\mathcal{C}$ uma categoria pequena e $F: \mathcal{C} \rightarrow$ Set um funtor, pode-se verificar que o limite $\left(l,\left(\psi_{c}\right)_{c \in \mathcal{C}_{0}}\right)$ de $F$ será dado por:

$$
\begin{gathered}
l=\left\{\left(x_{c}\right)_{c \in \mathcal{C}_{0}} \in \prod_{c \in \mathcal{C}_{0}} F_{0}(c) \mid F_{1}(f)\left(x_{c}\right)=x_{c^{\prime}} \text {, para toda } f: c \rightarrow c^{\prime} \text { em } \mathcal{C}_{1}\right\} \\
\psi_{d}: l \rightarrow F_{0}(d), \text { tal que } \psi_{d}\left(\left(x_{c}\right)_{c \in \mathcal{C}_{0}}\right)=x_{d}
\end{gathered}
$$

para toda $d \in \mathcal{C}_{0}$ e todo $\left(x_{c}\right)_{c \in \mathcal{C}_{0}} \in l$. Aliás, essa construção é semelhante à construção necessária para mostrar que podemos construir limites quaisquer com produtos e equalizadores.
} 
Agora, sejam $F, G$ prefeixes. Se existir o prefeixe exponenciação $G^{F}: \mathcal{C}^{o p} \rightarrow$ Set, então, pelo Lema de Yoneda e usando que a exponenciação é adjunta do produto, para todo $C \in \mathcal{C}_{0}$ teríamos:

$$
\left(G^{F}\right)_{0}(c) \cong \operatorname{Set}^{\mathcal{C}^{o p}}\left(\mathcal{C}^{o p}(c,-), G^{F}\right) \cong \operatorname{Set}^{\mathcal{C}^{o p}}(\mathcal{C}(-, c) \times F, G)
$$

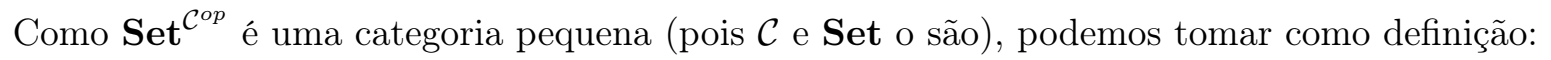

$$
\left(G^{F}\right)_{0}(c):=\operatorname{Set}^{\mathcal{C}^{o p}}(\mathcal{C}(-, c) \times F, G), \text { para todo } c \in \mathcal{C}_{0}
$$

$$
\begin{aligned}
\left(G^{F}\right)_{1}(f): & \left(G^{F}\right)_{0}(b) \rightarrow\left(G^{F}\right)_{0}(a), \text { para todo } f: b \rightarrow a \text { em } \mathcal{C}_{1}^{o p}, \text { tal que } \\
& \left(G^{F}\right)_{1}(f)(\eta):=\left(\mathcal{C}(-, f) \times i d_{F}\right) \circ \eta \text {, para toda } \eta: \mathcal{C}(-, b) \times F \Rightarrow G \text { em }\left(G^{F}\right)_{0}(b)
\end{aligned}
$$

onde $\mathcal{C}(-, f): \mathcal{C}(-, a) \Rightarrow \mathcal{C}(-, b)$ é a transformação natural tal que $\mathcal{C}(-, f)_{c}:=\mathcal{C}\left(c, f^{o p}\right)$ : $\mathcal{C}(c, a) \rightarrow \mathcal{C}(c, b)$, para todo $c \in \mathcal{C}_{0}$.

Agora, usando o Lema de Yoneda, que o funtor hom contravariante leva limites em colimites (e vice-versa), que o funtor $-\times G: \mathbf{S e t}^{\mathcal{C}^{o p}} \rightarrow \mathbf{S e t}^{\mathcal{C}^{o p}}$ preserva colimites ${ }^{2}$, e que todo prefeixe pode ser escrito como um colimite de funtores representáveis, obtemos as seguintes bijeções naturais:

$$
\begin{aligned}
\operatorname{Set}^{\mathcal{C o p}}(F \times G, H) & \cong \operatorname{Set}^{\mathcal{C}^{o p}}\left(\left(\operatorname{colim}_{i \in I} \mathcal{C}\left(-, c_{i}\right)\right) \times G, H\right) \\
& \cong \operatorname{Set}^{\mathcal{C}^{o p}}\left(\underset{i \in I}{\operatorname{colim}}\left(\mathcal{C}\left(-, c_{i}\right) \times G\right), H\right) \\
& \cong \lim _{i \in I} \operatorname{Set}^{\mathcal{C o p}}\left(\mathcal{C}\left(-, c_{i}\right) \times G, H\right) \\
& \cong \lim _{i \in I}\left(H^{G}\right)_{0}\left(c_{i}\right) \\
& \cong \lim _{i \in I} \operatorname{Set}^{\mathcal{C o p}}\left(\mathcal{C}\left(-, c_{i}\right), H^{G}\right) \\
& \cong \operatorname{Set}^{\mathcal{C}^{o p}}\left({ }_{i \in I} \mathcal{C o l i m}\left(-, c_{i}\right), H^{G}\right) \\
& \cong \operatorname{Set}^{\mathcal{C}^{o p}}\left(F, H^{G}\right)
\end{aligned}
$$

para todos prefeixes $F, G, H$.

Para o classificador de subobjeto, mais uma vez usamos o Lema de Yoneda: se existir o prefeixe $\Omega: \mathcal{C}^{o p} \rightarrow$ Set, então, usando o isomorfismo natural da proposição 3.2 .5 e a bijeção

\footnotetext{
${ }^{2}$ Isso porque, para cada $c \in \mathcal{C}_{0}$, o funtor $-\times G_{0}(c):$ Set $\rightarrow$ Set preserva colimites por ter um adjunto à direita (usando que Set é cartesianamente fechada), e colimites em Set $^{\mathcal{C}^{o p}}$ são computados ponto a ponto.
} 
entre subfuntores de $\mathcal{C}(-, c)$ e crivos em $c$ (proposição 2.3.5), para todo $c \in \mathcal{C}_{0}$ teríamos:

$$
\Omega_{0}(c) \cong \operatorname{Set}^{\mathcal{C}^{o p}}\left(\mathcal{C}^{o p}(c,-), \Omega\right) \cong \operatorname{Sub}(\mathcal{C}(-, c)) \cong\{\operatorname{crivos} \text { em } c\}
$$

Portanto, novamente usando que Set $^{\mathcal{C}^{o p}}$ é pequena, podemos definir (tomando a liberdade de omitir a bijeção entre os subfuntores e os crivos):

$$
\Omega_{0}(c):=\{\text { crivos em } c\}, \text { para todo } c \in \mathcal{C}_{0}
$$

$\Omega_{1}(f): \Omega_{0}(b) \rightarrow \Omega_{0}(a)$, para todo $f: b \rightarrow a$ em $\mathcal{C}_{1}^{o p}$, com

$$
\Omega_{1}(f)(S):=f^{*} S, \text { para todo } S \in \Omega_{0}(b)
$$

O prefeixe terminal 1 é dado por $\mathbf{1}_{0}(c)=\{*\}$, para todo $c \in \mathcal{C}_{0}$, então definimos o classificador de subobjeto $\top: \mathbf{1} \Rightarrow \Omega$ fazendo, para todo $c \in \mathcal{C}, \top_{c}(*)=S_{\mathcal{C}(-, c)}$, ou seja, o crivo formado por todas as flechas com codomínio $c$.

Dessa forma, seja $\mu: F \Rightarrow G$ um mono em Set $^{{ }^{\text {cop }}}$. Definimos sua flecha característica $\chi_{\mu}: G \Rightarrow \Omega$ fazendo, para todo $c \in \mathcal{C}_{0},\left(\chi_{\mu}\right)_{c}: G_{0}(c) \rightarrow \Omega_{0}(c)$, tal que:

$$
\left(\chi_{\mu}\right)_{c}(x)=\left\{f: c^{\prime} \rightarrow c \text { em } \mathcal{C}_{1} \mid G_{1}\left(f^{o p}\right)(x) \in F_{0}\left(c^{\prime}\right)\right\}
$$

para todo $x \in G_{0}(c)$, ou seja, vale $\left(\chi_{\mu}\right)_{c}(x)=\top_{c}(*)$ se, e somente se, $x \in F_{0}(c)$ (basta pensar no mono $\mu_{c^{\prime}}: F_{0}\left(c^{\prime}\right) \rightarrow G_{0}\left(c^{\prime}\right)$ como inclusão, pois estamos em Set). Portanto, temos o pullback:

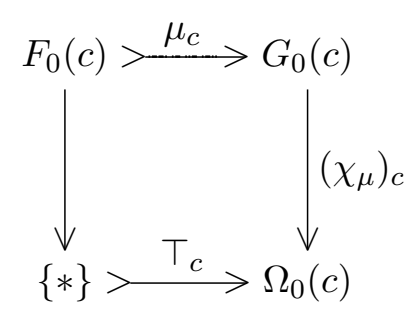

Observe que pela definição de crivo, para toda $f: c \rightarrow c^{\prime}$ em $\mathcal{C}_{1}$ e todo crivo $S$ em $c^{\prime}$, temos $f \in S$ se, e somente se, $f^{*} S=\top_{c}$. Assim, se $\eta: G \Rightarrow \Omega$ também satisfaz o pullback da flecha característica, para todo $x \in G_{0}(c)$ vale:

$$
f \in \eta_{c^{\prime}}(x) \Leftrightarrow \eta_{c}\left(F_{1}(f)(x)\right)=\top_{a} \Leftrightarrow G_{1}(f)(x) \in F_{0}(c)
$$

de forma que $\eta=\chi_{\mu}$, e a flecha característica é única. 
Exemplo 3.5.3. Seja $(L, \leq)$ um locale. Então, a categoria $\mathbf{S h}(L)$ dos feixes sobre $L$ é um topos. Dada uma categoria pequena $\mathcal{C}, \operatorname{como} \mathbf{S h}(L)$ é uma subcategoria de $\mathbf{S e t}^{L^{o p}}$ e os limites aí existem e são computados ponto a ponto (corolário 1.5.17), o limite de um funtor $\mathbf{F}: \mathcal{C} \rightarrow \mathbf{S h}(L)$ em cada $a \in L$ será dado pelo limite dos feixes $\mathbf{F}_{0}(c)$ calculados em $a$ (para cada $c \in \mathcal{C}_{0}$ ). Assim, uma família compatível para o limite define famílias compatíveis nos feixes $\mathbf{F}_{0}(c)$, e cada uma dessas admite, portanto, uma única colagem. A partir dessas colagens, podemos definir uma colagem única para a família compatível do limite, de forma que o limite também é um feixe e $\mathbf{S h}(L)$ é completa.

A exponenciação definida no exemplo anterior também serve aqui, isto é, se $F, G$ são feixes, então $G^{F}$ também é feixe. Porém, usando propriedades de locales pode-se obter uma descrição um pouco mais sucinta para a exponenciação: como as flechas $f: a \rightarrow b$ em $L_{1}$ (considerado como uma categoria) correspondem a $a \leq b$ e o produto corresponde ao ínfimo, dado $F$ um feixe o funtor $L(-, a) \times F$ corresponde ao funtor $F \uparrow_{a}$ dado por:

$$
\left(F \uparrow_{a}\right)_{0}(b)= \begin{cases}F_{0}(b) & , \text { se } b \leq a \\ \emptyset & , \text { caso contrário }\end{cases}
$$

para todo $b \in L$, e é fácil verificar que $F \uparrow_{a}$ também é um feixe. Ademais, dado outro feixe $G$, toda transformação natural $F \uparrow_{a} \Rightarrow G$ corresponde a uma transformação natural $F \uparrow_{a} \Rightarrow G \uparrow_{a}$, pois para todo $b \in L$, as componentes $\left(F \uparrow_{a}\right)_{0}(b) \rightarrow G_{0}(b)$ serão a função vazia se $b \not \leq a$. Dessa forma, a exponenciação pode ser dada por:

$$
\begin{gathered}
\left(G^{F}\right)_{0}(a)=N a t\left(F \uparrow_{a}, G \uparrow_{a}\right), \text { para todo } a \in L \\
\left(G^{F}\right)_{1} \text { é tal que } \eta \mapsto \eta \uparrow_{b}
\end{gathered}
$$

onde, se $b \leq a$ e dada uma transformação natural $\eta: F \uparrow_{a} \Rightarrow G \uparrow_{a}$, para todo $c \leq b$ as componentes $\eta_{c}$ constituem uma transformação natural $\eta \uparrow_{b}: F \uparrow_{b} \rightarrow G \uparrow_{b}$. Assim, é fácil verificar direto da definição de feixe que $G^{F}$ é um feixe quando $F, G$ o são.

Agora, dados $F, G, H$ feixes e $\eta: F \times G \Rightarrow H$ uma transformação natural, definimos uma transformação natural $\bar{\eta}: F \Rightarrow H^{G}$ fazendo, para todo $a \in L, \bar{\eta}_{a}: F_{0}(a) \rightarrow N a t\left(G \uparrow_{a}, H \uparrow_{a}\right)$ é a flecha tal que:

$$
\bar{\eta}_{a}(s): G \uparrow_{a} \Rightarrow H \uparrow_{a}, \text { para todo } s \in F_{0}(a),
$$




$$
\begin{aligned}
& \operatorname{com}\left(\bar{\eta}_{a}(s)\right)_{b}: G_{0}(b) \rightarrow H_{0}(b) \text { para todo } b \leq a, \\
& \text { onde }\left(\bar{\eta}_{a}(s)\right)_{b}\left(s^{\prime}\right)=\eta_{b}\left(\left.s\right|_{b} ^{a}, s^{\prime}\right) \text { para todo } s^{\prime} \in G_{0}(b)
\end{aligned}
$$

Reversamente, dada uma transformação natural $\theta: F \Rightarrow H^{G}$, definimos uma transformação natural $\breve{\theta}: F \times G \Rightarrow H$ fazendo, para todo $a \in L, \breve{\theta}_{a}: F_{0}(a) \times G_{0}(a) \rightarrow H_{0}(a)$ é a flecha tal que:

$$
\breve{\theta}_{a}\left(s, s^{\prime}\right)=\left(\theta_{a}(s)\right)_{a}\left(s^{\prime}\right)
$$

Essas construções $\eta \mapsto \bar{\eta}$ e $\theta \mapsto \breve{\theta}$ definem isomorfismos naturais inversos, de forma que:

$$
\operatorname{Nat}(F \times G, H) \cong N a t\left(F, H^{G}\right)
$$

e $\mathbf{S h}(L)$ é uma categoria cartesianamente fechada.

O classificador de subobjeto também pode ser obtido de Set $^{L^{o p}}$, e mais uma vez pode ser escrito de maneira mais simples. Como os crivos em um locale correspondem aos subconjuntos hereditários, podemos definir $\Omega: L^{o p} \rightarrow$ Set fazendo:

$$
\Omega_{0}(a)=\downarrow a, \text { para todo } a \in L
$$

$$
\text { se } b \leq a \text {, então } \Omega_{0}(a) \rightarrow \Omega_{0}(b) \text { é tal que } c \mapsto c \wedge b
$$

Assim definido, $\Omega$ é um feixe. Além disso, chamando de $\mathbf{1}$ o feixe terminal, definimos o mono $\top: \mathbf{1} \Rightarrow \Omega$ fazendo, para cada $a \in L$,

$$
\top_{a}: \mathbf{1}_{0}(a) \rightarrow \Omega_{0}(a), \operatorname{com}_{a}(*)=a
$$

Isso de fato define um classificador: para cada mono $\mu: S \Rightarrow F$ em $\mathbf{S h}(L)$, a flecha característica $\chi_{\mu}: F \Rightarrow \Omega$ será dada por:

$$
\left(\chi_{\mu}\right)_{a}: F_{0}(a) \rightarrow \Omega_{0}(a) \text {, para todo } a \in L, \text { com }
$$

$$
\left(\chi_{\mu}\right)_{a}(s)=\bigvee\left\{b \leq a \mid \text { existe } t \in S_{0}(b) \text { tal que } s \uparrow_{b}^{a}=\mu_{b}(t)\right\} \text {, para todo } s \in F_{0}(a)
$$

Exemplo 3.5.4. Seja $(\mathcal{C}, J)$ um sítio. Então, a categoria $\operatorname{Sh}(\mathcal{C}, J)$ dos feixes sobre $(\mathcal{C}, J)$ é um topos. O argumento que essa categoria é (finitamente) completa é semelhante ao exemplo anterior e novamente decorre do fato de ser subcategoria de $\mathbf{S e t}^{\mathcal{C}^{o p}}$, onde os limites são computados ponto a ponto. 
Além disso, para qualquer prefeixe $F$ e qualquer feixe $G$ sobre $(\mathcal{C}, J)$, a exponencial $G^{F}$ como definida para prefeixes é também um feixe. Isso é obtido usando a definição equivalente de feixes da proposição 2.3.15: dada uma transformação natural $\theta: \tilde{S} \Rightarrow G^{F}(\operatorname{com} S \in J(c)$ ), estender $\theta$ a uma transformação natural $\tilde{\theta}: \mathcal{C}(-, c) \Rightarrow G^{F}$ corresponde a estender a transformação natural $\breve{\theta}=\eta: \tilde{S} \times F \Rightarrow G$ a uma transformação natural $\tilde{\eta}: \mathcal{C}(-, c) \times F \Rightarrow G$. Usando que $G$ é feixe, essa segunda extensão será única, de forma que $G^{F}$ também será um feixe.

Para o classificador de subobjeto, usamos os crivos fechados. Dado $c \in \mathcal{C}_{0}$ e $S$ um crivo em $c$, dizemos que $S$ é $J$-fechado se, para toda $f: c^{\prime} \rightarrow c, f^{*} S \in J\left(c^{\prime}\right)$ implica $f^{*} S=S_{\mathcal{C}(-, c)}$. Com isso, podemos definir o subfuntor $\Omega_{J}$ de $\Omega$ tomando $\Omega_{J}(c)=\{\operatorname{crivos} J$-fechados em $c\}$. Todo crivo possui um $J$-fechamento (a intersecção dos crivos $J$-fechados que o contém), portanto pode-se definir uma colagem para os crivos fechados, de forma que $\Omega_{J}$ é um feixe. Além disso, se $G$ é um feixe, um subfuntor $F$ de $G$ é um feixe se, e somente se, a flecha característica de $F \longmapsto F$ se fatora por $\Omega_{J} \longmapsto \Omega$. Assim, $\Omega_{J}$ define um classificador de subobjeto em $\mathbf{S h}(\mathcal{C}, J)$.

Exemplo 3.5.5. Seja $(M, \cdot, e)$ um monoide. Para qualquer conjunto $X$, definimos uma ação de $M$ sobre $X$ como uma função $\lambda: M \times X \rightarrow X$ tal que

$$
\begin{gathered}
\lambda(e, x)=x, \text { para todo } x \in X \\
\lambda(m, \lambda(n, x))=\lambda(m \cdot n, x), \text { para todos } m, n \in M, x \in X
\end{gathered}
$$

Um $M$-set é definido como um par $(X, \lambda)$ onde $X$ é um conjunto e $\lambda$ é uma ação de $M$ sobre $X$ (observe que $(M, \cdot)$ também forma um $M$-set). Definimos morfismos de $M$-sets como flechas $f$ : $(X, \lambda) \rightarrow(Y, \mu)$ tais que $f: X \rightarrow Y$ é uma função equivariante, isto é, $f(\lambda(m, x))=\mu(m, f(x))$, para todo $(m, x) \in M \times X$. Assim, definimos a categoria $M$-Set $\operatorname{dos} M$-sets e seus morfismos.

Por outro lado, qualquer monoide $(M, \cdot, e)$ pode ser visto como uma categoria com um objeto $*$ e com flechas os elementos de $M$, definido a composição $m \circ n:=m \cdot n$ e a unidade como o elemento neutro e. Assim, podemos considerar a categoria de funtores $\mathbf{S e t}^{M}$.

Essas duas categorias são isomorfas, e os isomorfismos $\Phi: \operatorname{Set}^{M} \rightarrow M$-Set e $\Psi: M$-Set $\rightarrow$ Set $^{M}$ são dados por:

$\Phi_{0}(F):=\left(F_{0}(*), \lambda_{F}\right)$, para todo funtor $F: M \rightarrow$ Set, onde $\lambda$ é a ação dada por $\lambda_{F}(m, x):=F_{1}(m)(x)$, para todo $(m, x) \in M \times F_{0}(*)$ 
$\Phi_{1}(\eta):\left(F_{0}(*), \lambda_{F}\right) \rightarrow\left(G_{0}(*), \lambda_{G}\right)$, para toda transformação natural $\eta: F \Rightarrow G$, $\operatorname{com} \Phi_{1}(\eta):=\eta_{*}: F_{0}(*) \rightarrow G_{0}(*)$ (a única componente da transformação natural)

$\Psi_{0}(X, \lambda): M \rightarrow$ Set, para todo $M$-set $(X, \lambda)$, o funtor dado por:

$\left(\Psi_{0}(X, \lambda)\right)_{0}(*):=X$ e, para todo $m \in M,\left(\Psi_{0}(X, \lambda)\right)_{1}(m): X \rightarrow X$

é a flecha tal que $\left(\Psi_{0}(X, \lambda)\right)_{1}(m)(x):=\lambda(m, x)$, para todo $x \in X$

$\Psi_{1}(f): \Psi_{0}(X, \lambda) \Rightarrow \Psi_{0}(Y, \mu)$, para toda $f:(X, \lambda) \rightarrow(Y, \mu)$ em $M$-Set Se $_{1}$, onde a única componente da transformação natural é $\left(\Psi_{1}(f)\right)_{*}:=f: X \rightarrow Y$

Como já mostramos que $\operatorname{Set}^{\mathcal{C}^{o p}}$ é um topos para qualquer categoria pequena $\mathcal{C}$, em particular Set $^{M} \cong M$-Set também serão topoi.

Explicitamente, poderíamos também especificar a estrutura de topos de $M$-Set. Para todos $M$-sets $(X, \lambda)$ e $(Y, \mu)$, chamando $(E, \sigma):=(Y, \mu)^{(X, \lambda)}$, definimos a exponenciação fazendo:

$$
E:=M-\operatorname{Set}((M, \cdot) \times(X, \lambda),(Y, \mu))
$$

$$
\begin{aligned}
\sigma(m, f):(M, \cdot) \times(X, \lambda) \rightarrow(Y, \mu), & \text { para todo }(m, f) \in M \times E, \text { com } \\
& \sigma(m, f)(n, x):=f(n \cdot m, x) \text {, para todo }(n, x) \in M \times X
\end{aligned}
$$

Para o classificador, definimos $\Omega=\left(L_{M}, \omega\right)$, onde $L_{M}$ é o conjunto dos ideais à esquerda de $M$ e a ação $\omega: M \times L_{M} \rightarrow L_{M}$ é dada por $\omega(m, S)=\{n \in M \mid n \cdot m \in S\}$. Assim, o classificador de subobjeto será $\top: \mathbf{1} \rightarrow \Omega$ com $\top:\{*\} \rightarrow L_{M}$ a função equivariante $\top(*)=M$; e, dado um mono $f:(X, \lambda) \rightarrow(Y, \mu)$, visto como uma inclusão sua flecha característica $\chi_{f}:(Y, \mu) \rightarrow \Omega$ será dada pela função equivariante $\chi_{f}: Y \rightarrow L_{M}$ tal que:

$$
\chi_{f}(y)=\{m \in M \mid \mu(m, y) \in X\}, \text { para todo } y \in Y
$$

Para esses e outros exemplos, pode-se consultar a seção 5.2 de [Bor08c] e a seção A2.1 de [Joh02a]; para o exemplo do topos $M$-Set em particular, recomendamos [EM01]. 


\subsection{Tipos de topoi}

Façamos aqui um breve resumo sobre diferentes tipos de topoi; uma apresentação mais detalhada é feita em [Gol79], principalmente nas seções 5.4, 7.3 e 7.4.

Definição 3.6.1. Seja $\mathcal{C}$ uma categoria cartesianamente fechada. Dizemos que $\mathcal{C}$ é degenerada se $\mathcal{C} \simeq \mathbb{I}$, com $\mathbb{I}$ a categoria unitária (com um objeto e uma flecha). Observe que, nesse caso, $\mathcal{C}$ é um topos.

Definição 3.6.2. Definimos a flecha $\perp: \mathbf{1} \longmapsto \Omega$ (que é mono pois seu domínio é $\mathbf{1}$ ) como a flecha característica de $\mathrm{O}_{\mathbf{1}}=\mathrm{I}_{\mathbf{0}}: \mathbf{0} \rightarrow \mathbf{1}$ :

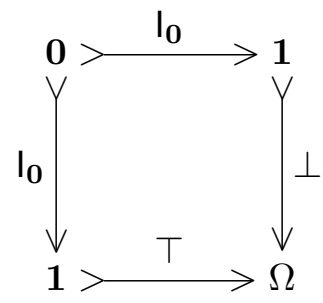

Observe que $\mathrm{I}_{\mathbf{0}}$ é, de fato, um mono, pois $\mathbf{0}$ é estrito (proposição 3.1.4): se existe $f: a \rightarrow \mathbf{0}$, então $\mathrm{O}_{a}: \mathbf{0} \rightarrow a$ é um iso. Nesse caso, para toda flecha $g: a \rightarrow \mathbf{0}$, como $\mathbf{0}$ é inicial, $g \circ \mathrm{O}_{a}=i d_{\mathbf{0}}$, ou $g=\mathrm{O}_{a}^{-1}$. Em outras palavras existe no máximo uma flecha $g: a \rightarrow \mathbf{0}$.

Proposição 3.6.3. Seja $\mathcal{C}$ uma categoria cartesianamente fechada com objeto terminal 1 e objeto inicial 0. Então, são equivalentes:

1. $\mathcal{C}$ é não degenerada;

2. $1 \neq 0$.

Proposição 3.6.4. Seja $\mathcal{E}$ um topos. Então, são equivalentes:

1. $\mathcal{E}$ é não degenerado;

2. $\top \neq \perp$.

Definição 3.6.5. Seja $\mathcal{E}$ um topos. Dizemos que $\mathcal{E}$ é bem pontuado quando $G=\{\mathbf{1}\}$ é gerador, isto é, para todos $f, g: a \rightarrow b$ em $\mathcal{E}_{1}, f \neq g$ se, e somente se, existe $p: \mathbf{1} \rightarrow a$ tal que $f \circ p \neq g \circ p$.

Exemplo 3.6.6. Set é bem pontuado, mas Set $\times$ Set não; por exemplo, não existe $p: 1 \rightarrow$ $(\emptyset,\{*\})$ que distingue o par de flechas $\left(i d_{\emptyset}, \top\right),\left(i d_{\emptyset}, \perp\right):(\emptyset,\{*\}) \rightarrow(\emptyset,\{0,1\})$. 
Proposição 3.6.7. Se $\mathcal{E}$ é um topos bem pontuado, então todo objeto não nulo a (isto é, a $¥ \mathbf{0}$ ) é não vazio (isto é, existe $p: \mathbf{1} \rightarrow a$ ).

Definição 3.6.8. Seja $\mathcal{E}$ um topos. Dizemos que $\mathcal{E}$ é bivalente se for não degenerado e $S u b(\mathbf{1}) \cong$ $\mathcal{E}(\mathbf{1}, \Omega) \cong\{\top, \perp\}$.

Exemplo 3.6.9. Novamente, Set é bivalente e Set $\times$ Set não, pois aí temos

$$
\operatorname{Sub}(\mathbf{1}) \cong\{(\top, \top),(\top, \perp),(\perp, \top),(\perp, \perp)\}
$$

Definição 3.6.10. Seja $\mathcal{E}$ um topos. Dizemos que $\mathcal{E}$ é clássico quando $[\top, \perp]: \mathbf{1}+\mathbf{1} \rightarrow \Omega$ é iso.

Exemplo 3.6.11. Set $\times$ Set é clássico, pois temos o iso:

$$
[(\perp, \perp),(\top, \top)]:\left(\left\{*, *^{\prime}\right\},\left\{*, *^{\prime}\right\}\right) \rightarrow(\{0,1\},\{0,1\})
$$

Exemplo 3.6.12. $M$-Set é um topos clássico se, e somente se, $\Omega=(\{\emptyset, M\}, \omega)$. Ou seja, exatamente quando os únicos ideais à esquerda são $\{\emptyset, M\}$, o que é equivalente a $M$ ser um grupo.

Definição 3.6.13. Seja $\mathcal{E}$ um topos. Dizemos que $\mathcal{E}$ é booleano se, para todo $a \in \mathcal{E}_{0},(\operatorname{Sub}(a), \leq)$ é uma álgebra de Boole.

Aliás, veremos no próximo capítulo que $(S u b(a), \leq)$ é sempre uma álgebra de Heyting. Por enquanto, listemos algumas equivalências da definição de um topos ser booleano.

Proposição 3.6.14. Seja $\mathcal{E}$ um topos. Então, são equivalentes:

1. $\mathcal{E}$ é booleano;

2. [T] possui complemento;

3. $[\perp]$ é complemento de $[\top]$;

4. $[\top] \cup[\perp] \cong\left[i d_{\Omega}\right]$ (o máximo em $\operatorname{Sub}(\Omega)$ );

5. $\mathcal{E}$ é clássico;

6. a injeção canônica $\iota_{1}: \mathbf{1} \rightarrow \mathbf{1}+\mathbf{1}$ é um classificador de subobjeto.

Proposição 3.6.15. Um topos $\mathcal{E}$ é bem pontuado se, e somente se, todo objeto não nulo é não vazio e $\mathcal{E}$ é clássico. 


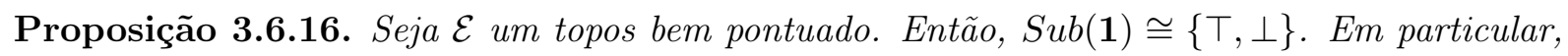
se $\mathcal{E}$ também for não degenerado, $\mathcal{E}$ será bivalente.

As demonstrações dos resultados dessa seção tornam-se mais naturais tendo disponível a lógica de topos.

\subsection{Topologias e feixes num topos}

Tal como definimos topologias e feixes no contexto de topoi de Grothendieck, também podese definir topologias e feixes no caso mais geral de um topos elementar. Apresentamos, a seguir, algumas definições e resultados básicos sobre essas noções. Para aprofundar-se nesse tópico, recomenda-se consultar o capítulo 9 de [Bor08c], as seções A4.4 e A4.5 de [Joh02a] ou o capítulo 21 de [Mc195].

Primeiramente, definamos a flecha conjunção $\wedge: \Omega \times \Omega \rightarrow \Omega$, que é a flecha característica da flecha $\langle\top, \top\rangle: \mathbf{1} \rightarrow \Omega$, obtida do produto. A razão para ser chamada dessa maneira ficará mais clara no próximo capítulo, quando corresponderá à interseção na álgebra de Heyting $(\operatorname{Sub}(A), \leq)$, mas precisamos dela aqui para definir a topologia.

Definição 3.7.1. Seja $\mathcal{E}$ um topos. Uma topologia (de Lawvere-Tierney) em $\mathcal{E}$ é uma flecha $j$ : $\Omega \rightarrow \Omega$ satisfazendo: $j \circ \top=\top$ (inflacionário), $j \circ j=j$ (idempotente) e $j \circ \wedge=\wedge \circ(j \times j)$ (preserva ou comuta com conjunção, e em particular é crescente), como nos diagramas comutativos abaixo:
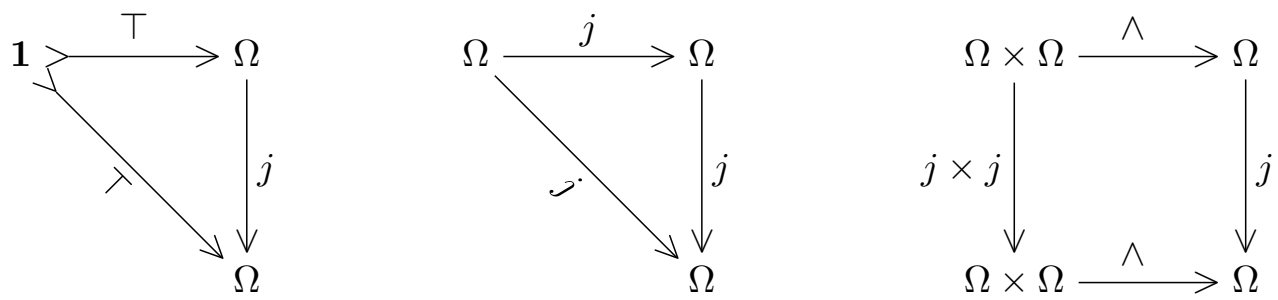

Nessa definição, é necessário fazer uma escolha do classificador de subobjeto. Porém, isso não é um problema, pois são sempre isomorfos (proposição 3.2.3).

Exemplo 3.7.2. Dois exemplos imediatos de topologias são $i d_{\Omega}: \Omega \rightarrow \Omega$ (chamada de topologia discreta) e $\top_{\Omega}=\top_{\circ} \circ \mathrm{I}_{\Omega}: \Omega \rightarrow \Omega$ (chamada de topologia caótica). Para um exemplo menos trivial, consideremos a negação $\neg=\chi_{\perp}$, a flecha característica de $\perp: 1 \longmapsto \Omega$ (novamente, essa definição está relacionada à estrutura de álgebra de Heyting de $S u b(A))$. Assim, a flecha da dupla negação $\neg \neg=\neg \circ \neg: \Omega \rightarrow \Omega$ é uma topologia. 
Proposição 3.7.3. Seja $\mathcal{C}$ uma categoria pequena. Então, existe uma bijeção entre as topologias de Grothendieck em $\mathcal{C}$ e as topologias $j: \Omega \rightarrow \Omega$ em Set $^{\mathcal{C}^{o p}}$.

Lembrando do exemplo 3.5.2 que o classificador de subobjeto em $\mathbf{S e t}^{\mathcal{C}^{o p}}$ é dado por $\Omega_{0}(c)=$ \{crivos em $c\}$ e $T_{c}(*)$ é o crivo maximal, as bijeções inversas são definidas da seguinte forma: dada uma topologia de Grothendieck $J$ em $\mathcal{C}$, defina $j^{J}: \Omega \rightarrow \Omega$ em $\operatorname{Set}^{\mathcal{C}^{o p}}$ como

$$
j_{c}^{J}(S):=\left\{g: a \rightarrow b \text { em } \mathcal{C}_{1} \mid g^{*} S \in J(a)\right\}
$$

(para todo $c \in \mathcal{C}_{0}$ e todo crivo $S$ em $c$ ). Por outro lado, a partir de uma topologia $j: \Omega \rightarrow \Omega \mathrm{em}$ Set $^{\mathcal{C}^{o p}}$, podemos definir uma topologia de Grothendieck $J_{j}$ em $\mathcal{C}$ fazendo, para cada $c \in \mathcal{C}_{0}$ :

$$
J_{j}(c):=\left\{S \in \Omega_{0}(c) \mid j_{c}(S)=\top_{c}(*)\right\}
$$

Definição 3.7.4. Sejam $\mathcal{E}$ um topos, $j$ uma topologia em $\mathcal{E}$ e $A \in \mathcal{E}_{0}$. Definimos o operador estável de fecho $\overline{(-)}^{j}: S u b(A) \rightarrow S u b(A)$ fazendo, para cada mono $m: a \longmapsto A, \bar{m}^{j}: \bar{a}^{j} \longmapsto \mathrm{A}$ é a flecha tal que $j \circ \chi_{m}=\chi_{\bar{m}^{j}}$.

É claro que isso está bem definido pois $f \sim g \Leftrightarrow \chi_{f}=\chi_{g}$ (proposição 3.2.3). Como seu nome sugere, esse operador possui algumas propriedades de fechamento:

Proposição 3.7.5. Sejam $\mathcal{E}$ um topos, $j$ uma topologia em $\mathcal{E}$ e $A \in \mathcal{E}_{0}$. Então, para todos $m: a \longmapsto A, n: b \longmapsto A$ e $g: c \rightarrow A$ em $\mathcal{E}_{1}$, vale:

1. $[m] \leq\left[\bar{m}^{j}\right]$ (inflacionário);

2. $\left[\bar{m}^{j}\right]=\left[\overline{\bar{m}}^{j}\right]$ (idempotente);

3. se $[m] \leq[n]$, então $\left[\bar{m}^{j}\right] \leq\left[\bar{n}^{j}\right]$ (crescente);

4. $\left(\bar{m}^{j}\right)^{\prime}={\overline{m^{\prime}}}^{j}$ (estabilidade por pullback), onde $\left(\bar{m}^{j}\right)^{\prime}$ é o pullback de $\bar{m}^{j}$ ao longo de g, e $m^{\prime}$ é o pullback de $m$ ao longo de $g$;

5. $\left[\overline{m \cup n}^{j}\right]=\left[\bar{m}^{j}\right] \cup\left[\bar{n}^{j}\right]$;

6. $\left[{\overline{i d_{A}}}^{j}\right]=\left[i d_{A}\right]$.

Os quatro primeiros itens dessa proposição, aliás, são a definição de um operador de fecho universal (e os dois últimos são consequência dos anteriores). 
Definição 3.7.6. Sejam $\mathcal{E}$ um topos, $j$ uma topologia em $\mathcal{E}, A \in \mathcal{E}_{0}$ e $m: a \longmapsto A$ em $\mathcal{E}_{1}$. Dizemos que $m$ é $j$-fechado se $\left[\bar{m}^{j}\right]=[m]$. Dizemos que $m$ é $j$-denso se $\left[\bar{m}^{j}\right]=\left[i d_{A}\right]$.

Proposição 3.7.7. Sejam $\mathcal{E}$ um topos, $j$ uma topologia em $\mathcal{E}, A \in \mathcal{E}_{0}$ e $m: a \longmapsto A$ em $\mathcal{E}_{1}$. Então,

1. $m$ é $j$-fechado se, e somente se, existe $\kappa: A \rightarrow j(\Omega)$ tal que $i m(j) \circ \kappa=\chi_{m}$;

2. $m$ é $j$-denso se, e somente se, existe $\rho: A \rightarrow p$ tal que $\check{j} \circ \rho=\chi_{m}$, onde $\check{j}: p \rightarrow \Omega$ é uma flecha correspondente a j pela bijeção $\operatorname{Sub}(\Omega) \cong \mathcal{E}(\Omega, \Omega)$ (lema 3.2.4).

Definição 3.7.8. Sejam $\mathcal{E}$ um topos, $j$ uma topologia em $\mathcal{E}$ e $F \in \mathcal{E}_{0}$. Dizemos que $F$ é $j$ separado se, para todo mono $j$-denso $m: a \longmapsto A$ em $\mathcal{E}_{1}, \mathcal{E}(m, F): \mathcal{E}(A, F) \rightarrow \mathcal{E}(a, F)$ é injetor. Dizemos que $F$ é um $j$-feixe se, para todo mono $j$-denso $m: a \longmapsto A$ em $\mathcal{E}_{1}, \mathcal{E}(m, F): \mathcal{E}(A, F) \rightarrow$ $\mathcal{E}(a, F)$ é bijetor.

Em outras palavras, $F$ é um $j$-feixe se para todos $m: a \longmapsto A j$-denso e $f: a \rightarrow F$, existe uma única $g: A \rightarrow F$ tal que $g \circ m=f$ (e $j$-separado se exigirmos apenas a unicidade, não a existência). Com isso, podemos definir a subcategoria $\operatorname{Sh}(\mathcal{E}, j)$ dos $j$-feixes de um topos $\mathcal{E}$.

Usando a proposição 3.7.3, pode-se mostrar que a noção de $j$-feixe para uma topologia $j: \Omega \rightarrow \Omega$ em $\mathbf{S e t}^{\mathcal{C}^{o p}}$ corresponde à noção de feixe sobre o sítio $\left(\mathcal{C}, J_{j}\right)$.

Exemplo 3.7.9. O objeto $j(\Omega)$ (obtido da imagem de $j$ ) é um feixe. Além disso, como $i d_{\mathbf{1}}$ : $\mathbf{1} \rightarrow \mathbf{1}$ é $j$-fechado, existe uma única $\top_{j}: \mathbf{1} \rightarrow j(\Omega)$ tal que $i m(j) \circ \top_{j}=\chi_{\mathbf{1}}=\top$, e tal $\top_{j}$ define um classificador de subobjeto em $\mathbf{S h}(\mathcal{E}, j)$.

Proposição 3.7.10. Sejam $\mathcal{E}$ um topos, $j$ uma topologia em $\mathcal{E}, F$ um $j$-feixe e $G \in \mathcal{E}_{0}$. Então:

1. se existe $s: G \longmapsto F$, então $G$ é feixe se, e somente se, s é $j$-fechado;

2. $F^{G}$ é um j-feixe.

Com isso, $\mathbf{S h}(\mathcal{E}, j)$ possui classificador de subobjeto e exponenciação, logo também é um topos.

Proposição 3.7.11. Seja $\mathcal{E}$ um topos. Então, $\mathbf{S h}(\mathcal{E}, \neg \neg)$ é sempre um topos booleano.

Em particular, quando $\mathcal{E}=\mathbf{S h}(L)$, para um locale $L, \mathbf{S h}(\mathcal{E}, \neg \neg) \simeq \mathbf{S h}(\operatorname{Reg}(L))$, onde $\operatorname{Reg}(L)=\{a \in L \mid a=\neg \neg a\}$, o conjunto dos elementos regulares de $L$, que é sempre uma álgebra de Boole completa. 


\section{Capítulo 4}

\section{Lógica de topos}

O objetivo deste capítulo será apresentar a lógica de topos. Começamos descrevendo a estrutura de $S u b(A)$, para qualquer objeto $A$ no topos, como uma álgebra de Heyting (e, como pode ser visto no apêndice A, álgebras de Heyting são modelos algébricos para lógica intuicionista proposicional). Em particular, descrevemos a correspondência entre as operações lógicas em $S u b(A)$ e as operações em $\Omega^{A}$, que será, portanto, um "objeto álgebra de Heyting" no topos. Desenvolvemos essa parte em detalhes pois, na bibliografia, essa correspondência geralmente não é feita de forma explícita ou minuciosa.

Além da estrutura de álgebra de Heyting dos subobjetos, também veremos que podemos definir quantificadores usando a adjunção $\Sigma_{f} \dashv f^{\star} \dashv \Pi_{f}$. Isso nos permitirá, pois, considerar uma "lógica interna" do topos. Formalizamos tal lógica interna através da linguagem de MitchellBénabou, e, para finalizar, introduzimos a semântica de Kripke-Joyal, que serve de interface semântica entre a descrição (sintática) interna de objetos usando a linguagem de Mitchell-Bénabou e a descrição externa.

As principais referências para este capítulo são [Bor08c] (capítulo 6), [Gol79] (capítulos 5 a 7), [LS86] (parte II) e [Mcl95] (capítulos 14 a 16).

Primeiramente, observe que um topos satisfaz ambas as condições para obter as descrições concretas da intersecção e da união, portanto vale:

Teorema 4.0.1. Sejam $\mathcal{E}$ um topos com classificador de subobjeto $\top: \mathbf{1} \rightarrow \Omega$ e $A \in \mathcal{E}_{0}$. Então, $(\operatorname{Sub}(A), \leq)$ é um reticulado limitado, com unidade $\left[i d_{A}\right]$ e zero $\left[\mathrm{O}_{A}\right]$.

Demonstração. Um topos é finitamente completo por definição (logo possui pullbacks), e a exis- 
tência da fatoração epi-mono foi feita no teorema 3.4.8, portanto pelos teoremas 1.6.6 e 1.6.5 existe o supremo (a união) e o ínfimo (a intersecção). Agora, seja $\alpha: a \longmapsto A$ em $\mathcal{E}_{1}$. Pela definição da identidade $i d_{A}$, temos $i d_{A} \circ \alpha=\alpha, \log \alpha \preceq \preceq i d_{A}$. E pela unicidade da flecha $\mathrm{O}_{A}$, temos $\mathrm{O}_{A}=\alpha \circ \mathrm{O}_{A}, \operatorname{logo} \mathrm{O}_{A} \preceq \alpha$.

Nas próximas duas seções, definiremos operações de conjunção e união em um classificador de objetos $\Omega$ e mostraremos como elas se relacionam com essas operações definidas acima para subobjetos.

No restante deste capítulo, trataremos sempre de um topos $\mathcal{E}$ com classificador de subobjeto $\top: \mathbf{1} \rightarrow \Omega$ e de um objeto $A \in \mathcal{E}_{0}$. O objeto inicial de $\mathcal{E}$ será denotado por $\mathbf{0}$ (e o inicial por $\mathbf{1}$, como já foi sugerido na notação do classificador).

\subsection{Conjunção}

Definição 4.1.1. Definimos a conjunção $\wedge: \Omega \times \Omega \rightarrow \Omega$ como a flecha característica de $\langle\top, \top\rangle: \mathbf{1} \longmapsto \Omega \times \Omega$ (que é naturalmente um mono pois seu domínio é $\mathbf{1}$ ):

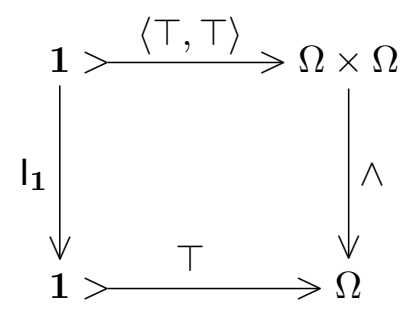

Definição 4.1.2. Dados $\alpha: a \longmapsto A$ e $\beta: b \longmapsto A$ em $\mathcal{E}_{1}$, o mono $\alpha$ ก $\beta$ é definido como o pullback do mono $\top$ ao longo de $\chi_{\alpha} \wedge \chi_{\beta}:=\wedge \circ\left\langle\chi_{\alpha}, \chi_{\beta}\right\rangle$ :

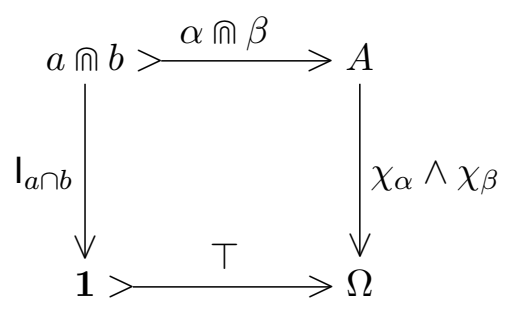

Ou seja, $\chi_{\alpha} \wedge \chi_{\beta}=\chi_{\alpha \boxplus \beta}$.

Exemplo 4.1.3. Em Set, $\wedge:\{0,1\} \times\{0,1\} \rightarrow\{0,1\}$ é a função tal que $\wedge(x, y)=1$ se, e somente se, $x=y=1$. Assim, dados $a, b \subseteq A$ e $z \in A,\left(\chi_{a} \wedge \chi_{b}\right)(z)=1$ se, e só se, $\chi_{a}(z)=\chi_{b}(z)=1$, isto é, $z \in a \cap b$; ou seja, $\chi_{a} \wedge \chi_{b}$ corresponde à função característica de $a \cap b \subseteq A$. 
Teorema 4.1.4. Sejam $\alpha: a \longmapsto A$ e $\beta: b \longmapsto A$ em $\mathcal{E}_{0}$ com pullback:

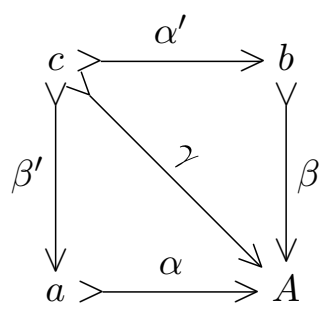

como no teorema 1.6.5. Então, $\chi_{\gamma}=\chi_{\alpha \circledast \beta}$, de forma que $\gamma \sim \alpha \pitchfork \beta$ e temos o pullback:

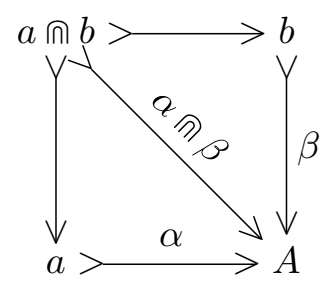

Em outras palavras, $f \cap g=[f \cap g]$.

Demonstração. Nosso objetivo será mostrar que

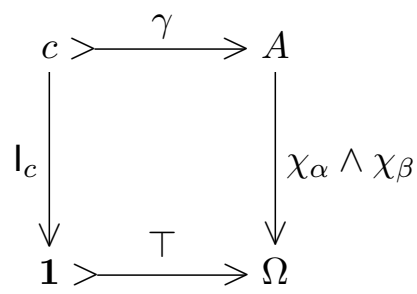

é um pullback, de modo que, pela unicidade na definição do classificador de subobjeto, tenhamos

$$
\chi_{\alpha} \wedge \chi_{\beta}=\chi_{\alpha \cap \beta}=\chi_{\gamma}
$$

Para tanto, usaremos o lema do pullback em:

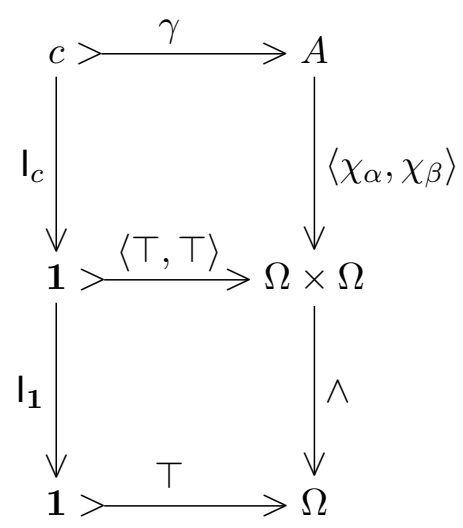

O quadrado inferior é um pullback pela própria definição de $\wedge$. Quanto ao quadrado superior, 
sejam $\delta: d \rightarrow A$ e $\mathrm{I}_{d}: d \rightarrow \mathbf{1}$ tais que $\langle\top, \top\rangle \circ \mathrm{I}_{d}=\left\langle\chi_{\alpha}, \chi_{\beta}\right\rangle \circ \delta$. Devemos achar uma flecha única $\kappa: d \rightarrow c$ tal que o diagrama abaixo fique comutativo:

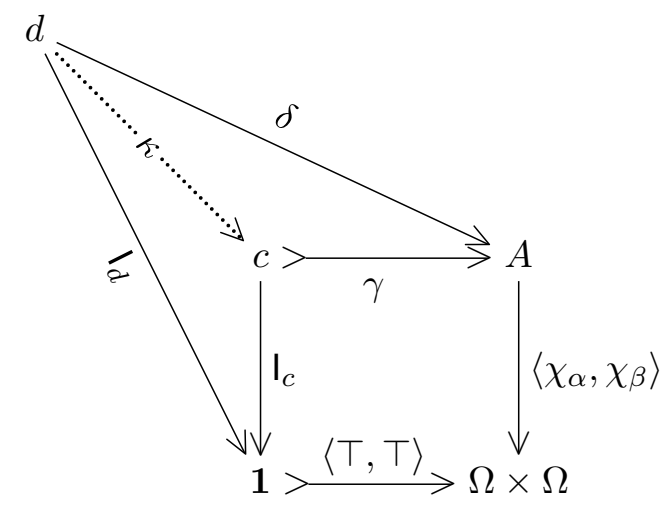

Primeiramente, note que $\langle T, \top\rangle \circ \mathbf{I}_{d}=\left\langle\chi_{\alpha}, \chi_{\beta}\right\rangle \circ \delta$ implica $T \circ \mathbf{I}_{d}=\chi_{\alpha} \circ \delta \mathrm{e} \top \circ \mathbf{I}_{d}=\chi_{\beta} \circ \delta$. Logo, usando a definição das funções características pelo pullback, temos as flechas únicas $\kappa_{a}, \kappa_{b}$ nos diagramas:
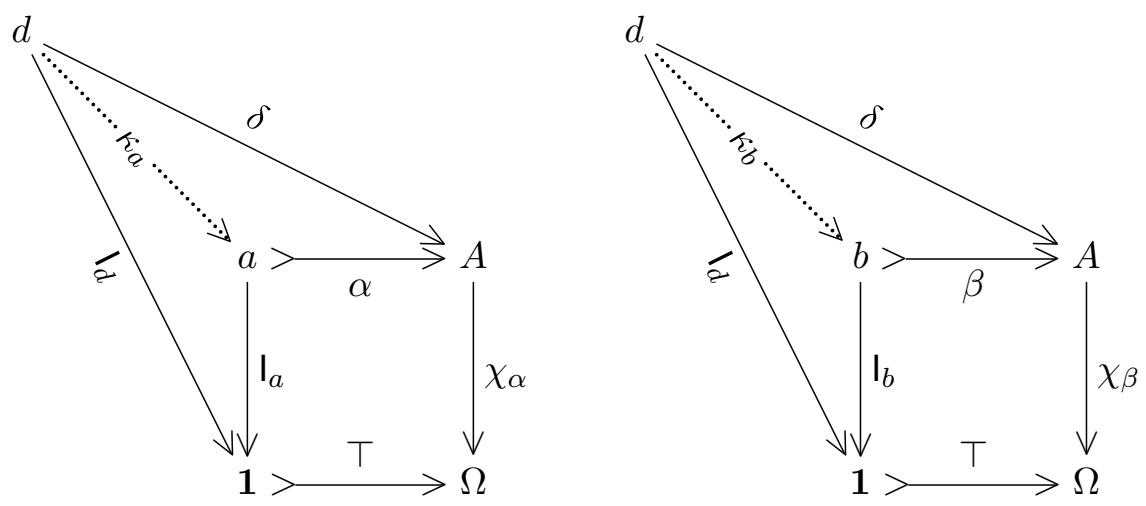

ou seja, $\alpha \circ \kappa_{a}=\delta=\beta \circ \kappa_{b}$. Agora, pelo pullback da hipótese, temos que existe uma única flecha $\kappa$ tal que fica comutativo:

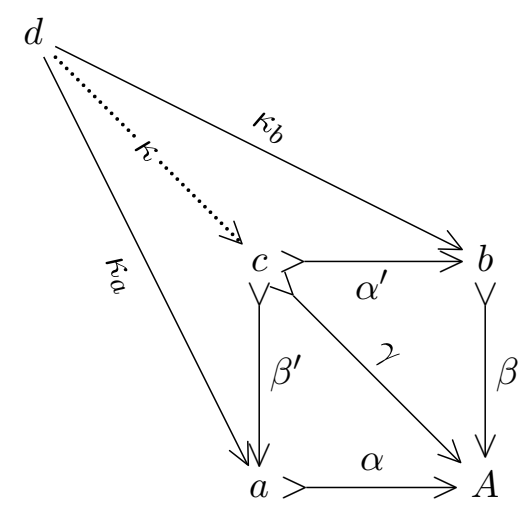

Com isso, $\gamma \circ \kappa=\alpha \circ \kappa_{a}=\delta$, e é claro que $\mathrm{I}_{d}=\mathrm{I}_{c} \circ \kappa$ pois o codomínio é $\mathbf{1}$. Logo, tal $\kappa$ satisfaz as hipóteses que queríamos. Falta mostrar unicidade. Seja $\rho$ tal que $\mathrm{I}_{c} \circ \rho=\mathrm{I}_{d}$ e $\gamma \circ \rho=\delta$. Observe que $\gamma$ é mono, por ser composição de monos, então $\gamma \circ \rho=\delta=\gamma \circ \kappa$ implica em $\rho=\kappa$. 


\subsection{Disjunção}

Primeiramente, para todo $a \in \mathcal{E}_{0}$, denotamos por $\top_{a}$ a flecha $\top_{a}:=\top \circ \mathrm{I}_{a}$

Definição 4.2.1. Definimos a disjunção $\vee: \Omega \times \Omega \rightarrow \Omega$ como a flecha característica da imagem (portanto um mono) de $\varphi=\left[\left\langle\top_{\Omega}, i d_{\Omega}\right\rangle,\left\langle i d_{\Omega}, \top_{\Omega}\right\rangle\right]: \Omega+\Omega \rightarrow \Omega \times \Omega$ :
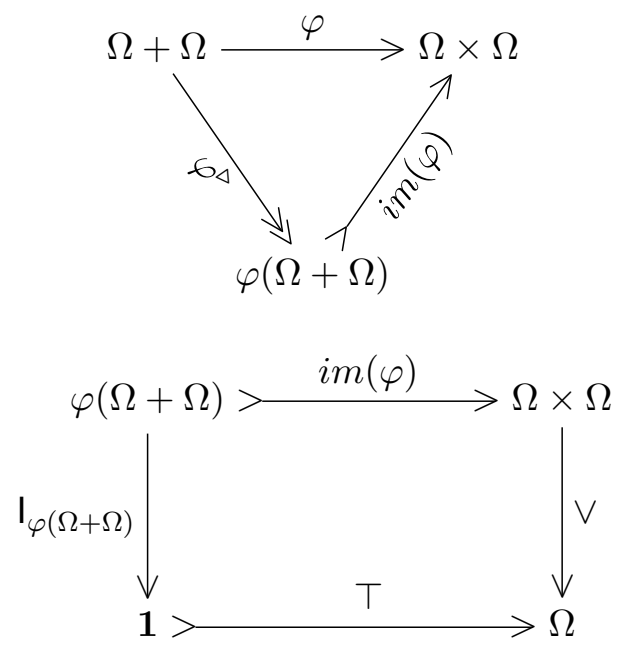

Definição 4.2.2. Dados $\alpha: a \longmapsto A$ e $\beta: b \longmapsto A$ em $\mathcal{E}_{1}$, o mono $\alpha \uplus \beta$ é definido como o pullback do mono $\top$ ao longo de $\chi_{\alpha} \vee \chi_{\beta}:=\vee \circ\left\langle\chi_{\alpha}, \chi_{\beta}\right\rangle$ :

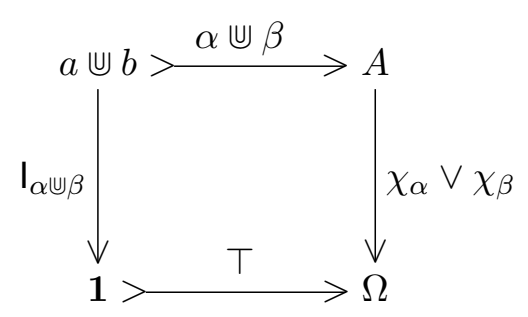

Ou seja, $\chi_{\alpha} \vee \chi_{\beta}=\chi_{\alpha \uplus \beta}$.

Exemplo 4.2.3. Em Set, $\vee:\{0,1\} \times\{0,1\} \rightarrow\{0,1\}$ é a função tal que $\vee(x, y)=1$ se, e somente se, $(x, y) \in\{(1,1),(1,0),(0,1)\}$. Assim, dados $a, b \subseteq A$ e $z \in A,\left(\chi_{a} \vee \chi_{b}\right)(z)=1$ se, e só se, $z \in a \cap b$ ou $(z \in a$ e $z \notin b)$ ou $(z \notin a$ e $z \in b)$, isto é, $z \in a \cup b$; ou seja, $\chi_{a} \vee \chi_{b}$ corresponde à função característica de $a \cup b \subseteq A$.

Ao contrário da conjunção, a correspondência não será tão imediata, e requer alguns resultados adicionais.

Proposição 4.2.4. 1. $\top_{\Omega} \circ \top=\top$;

2. Para qualquer $\alpha: a \longmapsto A$ em $\mathcal{E}_{1}$, vale:

(a) $\chi_{\alpha} \circ \alpha=\top_{\Omega} \circ \chi_{\alpha} \circ \alpha$ 
(b) $\top_{\Omega} \circ \chi_{\alpha}=\top \circ \mathbf{I}_{A}$, em particular $\top_{\Omega} \circ \chi_{\alpha}=\top_{\Omega} \circ \chi_{\beta}$ para todo $\beta: b \longmapsto A$ em $\mathcal{E}_{1}$.

Demonstração. 1. Verifiquemos que o diagrama abaixo é comutativo.

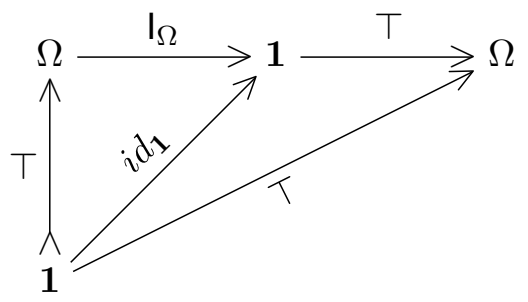

Como 1 é terminal, $\mathrm{I}_{\Omega} \circ \top=i d_{\mathbf{1}}$, e pela definição da flecha identidade, $T \circ i d_{\mathbf{1}}=\top$. Logo, $\top \circ\left(\mathrm{I}_{\Omega} \circ \top\right)=\top \circ i d_{\mathbf{1}}=\top$, donde obtemos $\top \circ \top_{\Omega}=\top$ usando a definição de $T_{\Omega}$.

2. Colaremos o diagrama do item anterior na definição da flecha característica $\chi_{\alpha}$ :

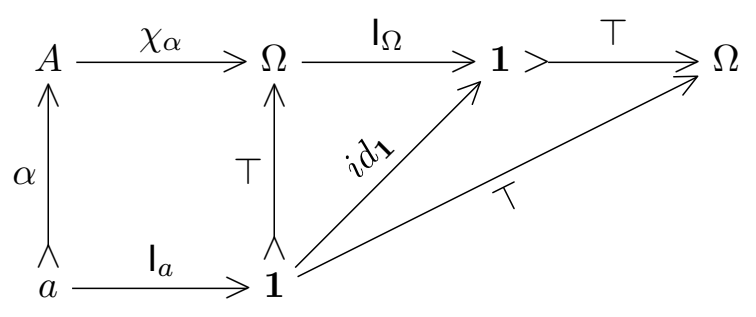

Explicitamente:

$$
\chi_{\alpha} \circ \alpha=\top \circ \mathbf{I}_{a}=\left(\top_{\Omega} \circ \top\right) \circ \mathbf{I}_{a}=T_{\Omega} \circ\left(\top \circ \mathbf{I}_{a}\right)=T_{\Omega} \circ \chi_{\alpha} \circ \alpha
$$

donde sai o item (a). Para o item (b), olhamos a linha superior do diagrama: como 1 é terminal, $\mathrm{I}_{\Omega} \circ \chi_{\alpha}=\mathrm{I}_{A}$, logo

$$
\top \circ \mathrm{I}_{A}=\top \circ\left(\mathrm{I}_{\Omega} \circ \chi_{\alpha}\right)=\left(\top \circ \mathrm{I}_{\Omega}\right) \circ \chi_{\alpha}=\top_{\Omega} \circ \chi_{\alpha}
$$

Como isso vale para todo mono com codomínio $A$, em particular temos $\top_{\Omega} \circ \chi_{\beta}=\top \circ \mathrm{I}_{A}=$ $\top_{\Omega} \circ \chi_{\alpha}$.

Proposição 4.2.5. Seja $\alpha: a \longmapsto A$ em $\mathcal{E}_{1}$. Então, $\alpha$ é um equalizador de $\chi_{\alpha} e \top_{A}$.

Essa proposição é resultado da demonstração da proposição 3.2.3.

Corolário 4.2.6. Seja $\alpha: a \longmapsto A$ em $\mathcal{E}_{1}$. Então, $\alpha$ é um equalizador de $\chi_{\alpha} e \top_{\Omega} \circ \chi_{\beta}$, para todo $\beta: b \longmapsto A$ em $\mathcal{E}_{1}$. 
Demonstração. Consequência imediata das duas proposições anteriores, pois

$$
\top_{\Omega} \circ \chi_{\beta}=\top \circ \mathrm{I}_{A}=\top_{A}
$$

Lema 4.2.7. Suponha que o diagrama

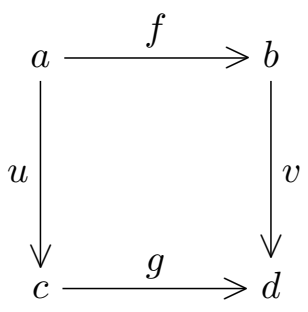

seja um pullback. Então, existe uma flecha $h: f(a) \rightarrow g(c)$ tal que o quadrado da direita abaixo é um pullback:

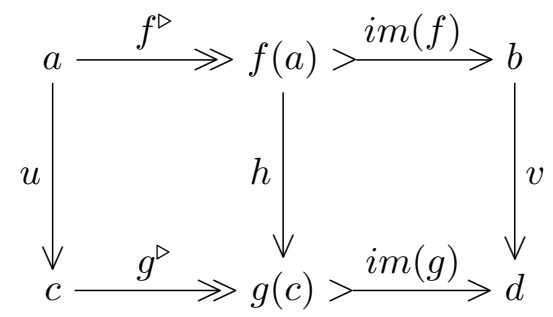

Demonstração. Primeiramente, façamos o pullback de $i m(g)$ e $v$ :

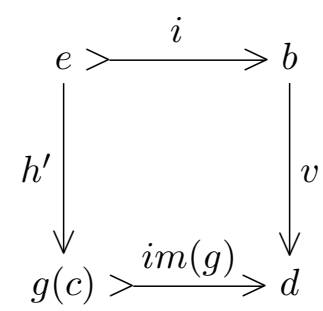

$i$ é mono pois $i m(g)$ é mono. Como, por hipótese, $v \circ f=g \circ u=i m(g) \circ g^{\triangleright} \circ u$, existe uma única $f^{\prime}$ tal que fica comutativo o diagrama:

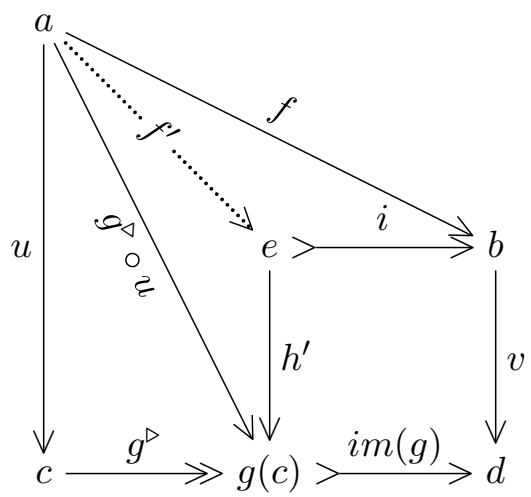


Agora, por hipótese, o retângulo de vértices $a, b, c, d$ é um pullback, e o quadrado de vértices $e, b, d, g(c)$ é pullback por definição. Logo, pelo lema do pullback:

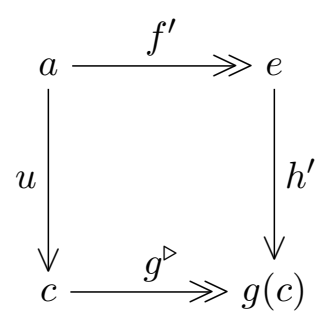

também é um pullback, e $f^{\prime}$ é epi pois $g^{\triangleright}$ também é (corolário 3.4.6). Portanto, $i \circ f^{\prime}$ é uma fatoração epi-mono de $f$, e existe um único iso $k: e \rightarrow f(a)$ tal que o diagrama abaixo fica comutativo:

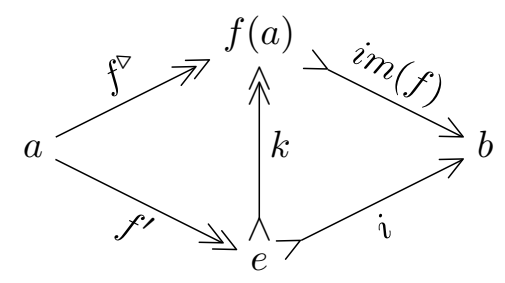

Assim, podemos definir $h=h^{\prime} \circ k^{-1}$, que satisfaz o desejado. De fato,

$$
i m(g) \circ h=i m(g) \circ h^{\prime} \circ k^{-1}=v \circ i \circ k^{-1}=v \circ i m(f)
$$

Além disso, para todas $\xi_{1}: x \rightarrow g(c)$ e $\xi_{2}: x \rightarrow b$ tais que $i m(g) \circ \xi_{1}=v \circ \xi_{2}$, existe uma única $\rho: x \rightarrow e$ tal que $h^{\prime} \circ \rho=\xi_{1}$ e $i \circ \rho=\xi_{2}$. Isto é, $h \circ(k \circ \rho)=\xi_{1}$ e $i m(f) \circ(k \circ \rho)=\xi_{2}$, como no diagrama:

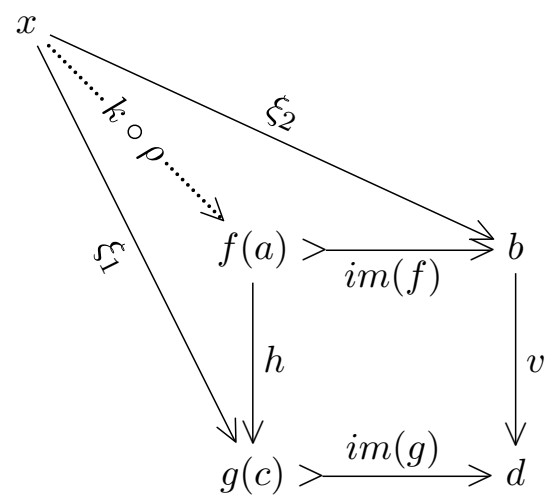

Para a unicidade: se existe $\rho^{\prime}: x \rightarrow f(a)$ tornando o diagrama comutativo, então

$$
i m(f) \circ(k \circ \rho)=\xi_{2}=i m(f) \circ \rho^{\prime}
$$

Como $i m(f)$ é mono, concluímos que $\rho^{\prime}=k \circ \rho$ 
Teorema 4.2.8. Sejam $\alpha: a \longmapsto A$ e $\beta: b \longmapsto A$ em $\mathcal{E}_{1}$ e $\gamma$ a flecha imagem de $[\alpha, \beta]: a+b \rightarrow A$ :

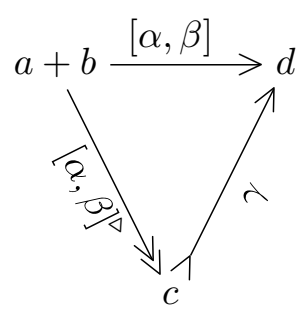

como no teorema 1.6.6. Então, $\gamma$ tem $\chi_{\alpha} \vee \chi_{\beta}$ como flecha característica, de forma que $\gamma \sim \alpha \uplus \beta$ e temos a fatoração epi-mono:

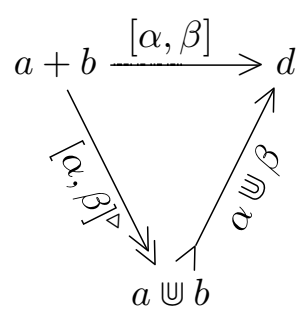

Em outras palavras, $f \cup g=[f \uplus g]$.

Demonstração. Comecemos mostrando que o diagrama abaixo é um pullback:

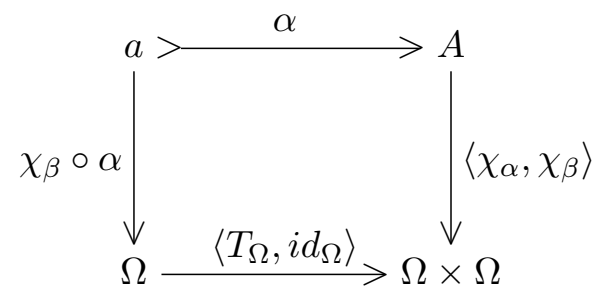

Para comutatividade, primeiro usamos a proposição 4.2 .4 para obter $\left(T_{\Omega} \circ \chi_{\beta}\right) \circ \alpha=\left(T_{\Omega} \circ\right.$ $\left.\chi_{\alpha}\right) \circ \alpha=\chi_{\alpha} \circ \alpha$, enquanto $\chi_{\beta} \circ \alpha=i d_{\Omega} \circ \chi_{\beta} \circ \alpha$ vale pela própria definição de $i d_{\Omega}$. Portanto, $\left\langle\chi_{\alpha}, \chi_{\beta}\right\rangle \circ \alpha=\left\langle T_{\Omega}, i d_{\Omega}\right\rangle \circ \chi_{\beta} \circ \alpha$.

Agora, sejam $\delta: d \rightarrow A$ e $\xi: d \rightarrow \Omega$ tais que $\left\langle\chi_{\alpha}, \chi_{\beta}\right\rangle \circ \delta=\left\langle T_{\Omega}, i d_{\Omega}\right\rangle \circ \xi$. Devemos achar $\kappa: d \rightarrow a$ que torne o diagrama comutativo:

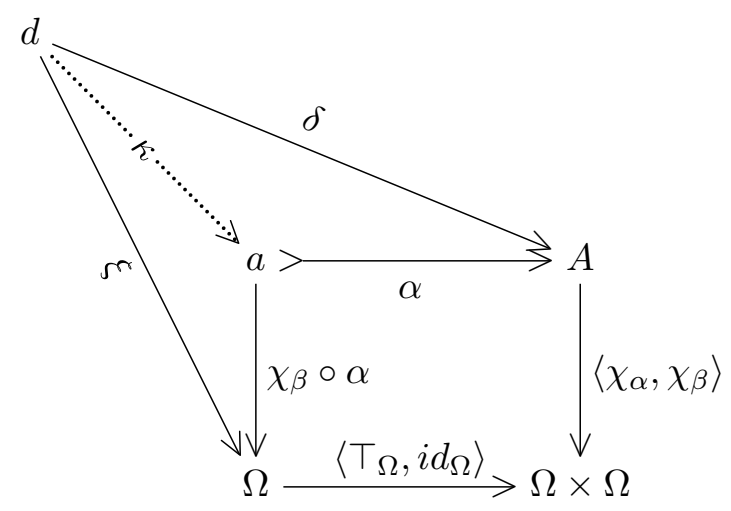


Note que de $\left\langle\chi_{\alpha}, \chi_{\beta}\right\rangle \circ \delta=\left\langle T_{\Omega}, i d_{\Omega}\right\rangle \circ \xi$ obtemos $\chi_{\alpha} \circ \delta=\top_{\Omega} \circ \xi$ e $\chi_{\beta} \circ \delta=\xi$, portanto $\chi_{\alpha} \circ \delta=\top_{\Omega} \circ \chi_{\beta} \circ \delta$. Daí, como sabemos do corolário 4.2.6 que $\alpha$ equaliza $\chi_{\alpha} \mathrm{e} T_{\Omega} \circ \chi_{\beta}$, obtemos a flecha única $\kappa: d \rightarrow a:$

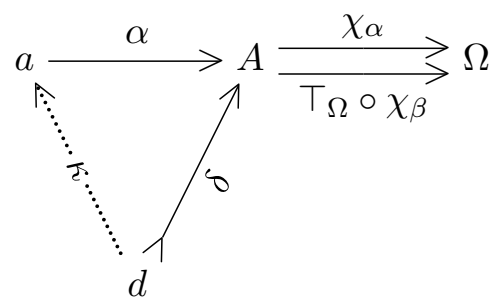

de forma que $\delta=\alpha \circ \kappa$. Ademais, $\chi_{\beta} \circ \alpha \circ \kappa=\chi_{\beta} \circ \delta=\xi$, como queríamos. A unicidade decorre facilmente de $\alpha$ ser mono: seja $\rho: d \rightarrow a$ tal que $\alpha \circ \rho=\delta=\alpha \circ \kappa$, então $\rho=\kappa$.

De forma análoga, pode-se mostrar que o quadrado da direita do diagrama abaixo também é um pullback:

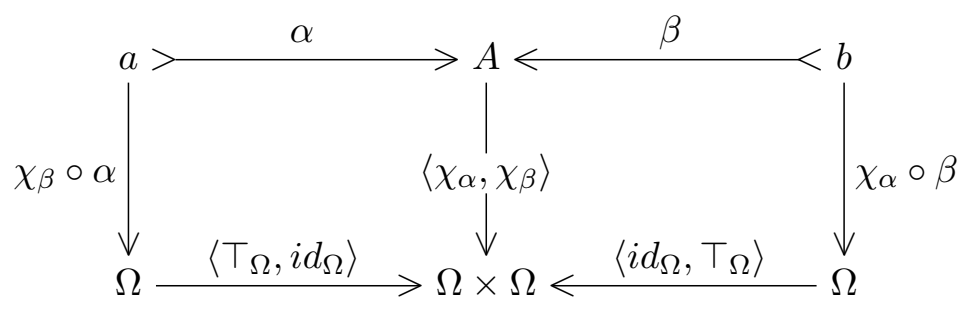

Usando que coprodutos são estáveis sob pullbacks num topos (corolário 3.4.7), obtemos o pullback:

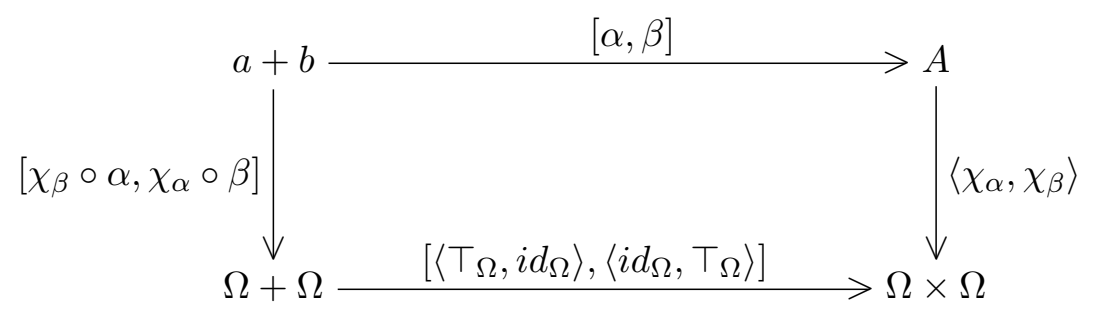

Com isso, pelo lema anterior, chamando $\left[\left\langle\top_{\Omega}, i d_{\Omega}\right\rangle,\left\langle i d_{\Omega}, \top_{\Omega}\right\rangle\right]$ de $\varphi$, e $[\alpha, \beta](a+b)$ de $c$ e $i m([\alpha, \beta])$ de $\gamma$ como no enunciado, existe $h: c \rightarrow \varphi(\Omega+\Omega)$ tal que o quadrado abaixo é um pullback:

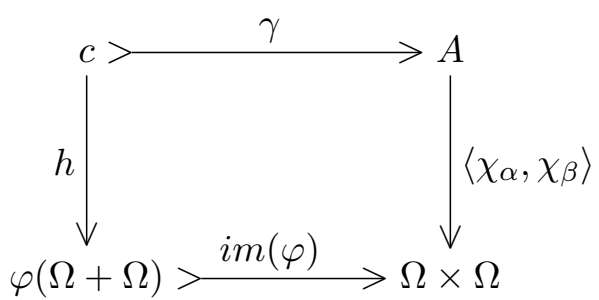


Por outro lado, como $\vee: \Omega \times \Omega \rightarrow \Omega$ é a flecha característica de $i m(\varphi)$, temos o pullback:

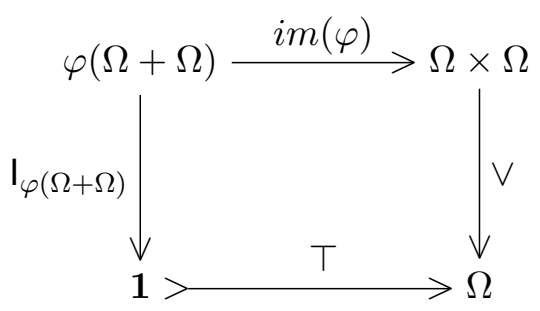

Assim, juntando ambos esses quadrados, o lema do pullback garante que o diagrama abaixo também seja um pullback:

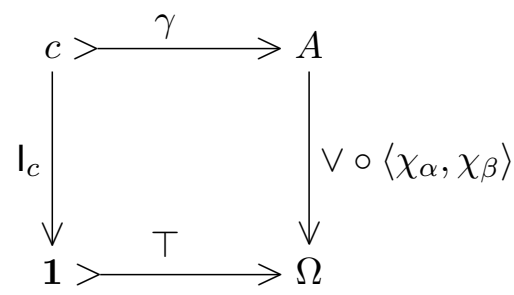

de forma que, pela unicidade da função característica, $\chi_{\gamma}=\vee \circ\left\langle\chi_{\alpha}, \chi_{\beta}\right\rangle$, como desejado.

\subsection{Implicação}

Definição 4.3.1. Definimos a implicação $\Leftrightarrow: \Omega \times \Omega \rightarrow \Omega$ como a flecha característica de $e: \preccurlyeq \rightarrow \Omega \times \Omega$, o equalizador de $\wedge: \Omega \times \Omega \rightarrow \Omega$ e $\pi_{1}: \Omega \times \Omega \rightarrow \Omega$ (a projeção na primeira coordenada):

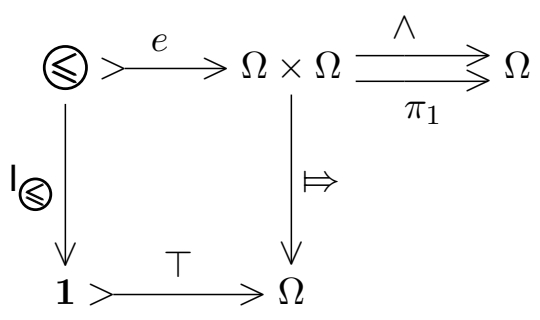

Definição 4.3.2. Dados $\alpha: a \longmapsto A$ e $\beta: b \longmapsto A$ em $\mathcal{E}_{1}$, o mono $\alpha \Rightarrow \beta$ é definido como o pullback do mono $\top$ ao longo de $\chi_{\alpha} \Leftrightarrow \chi_{\beta}:=\models \circ\left\langle\chi_{\alpha}, \chi_{\beta}\right\rangle$ :

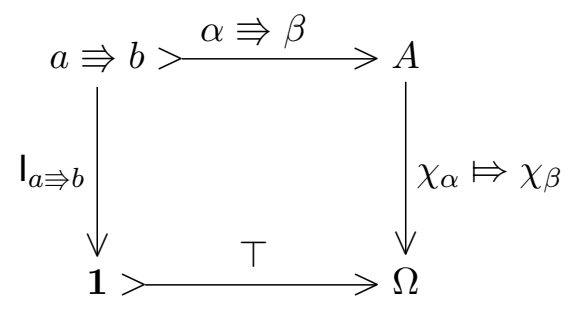

Ou seja, $\chi_{\alpha} \Leftrightarrow \chi_{\beta}=\chi_{\alpha \Rightarrow \beta}$ 
Exemplo 4.3.3. Em Set, $\mapsto:\{0,1\} \times\{0,1\} \rightarrow\{0,1\}$ é a função característica de $\preccurlyeq=$ $\{(0,0),(0,1),(1,1)\} \subseteq\{0,1\} \times\{0,1\}$. Assim, dados $a, b \subseteq A$ e $z \in A,\left(\chi_{a} \mapsto \chi_{b}\right)(z)=1$ se, e só se, $z \in a$ implica em $z \in b$.

Lema 4.3.4. Sejam $\alpha: a \longmapsto A, \beta: b \longmapsto A$ e $\gamma: c \longmapsto A$ em $\mathcal{E}_{1}$. Então:

1. $\alpha \cap \gamma=\beta \cap \gamma$ se, e somente se, $\chi_{\alpha} \circ \gamma=\chi_{\beta} \circ \gamma$;

2. $\chi_{\alpha} \wedge \chi_{\gamma} \sim \chi_{\beta} \wedge \chi_{\beta}$ se, e somente se, $\chi_{\alpha} \circ \gamma=\chi_{\beta} \circ \gamma$;

3. $\alpha \cap \gamma \leq[\beta]$ se, e somente se, $\chi_{\alpha \cap \beta} \circ \gamma=\chi_{\alpha} \circ \gamma$.

Demonstração. $\quad$ 1. Considere os diagramas:
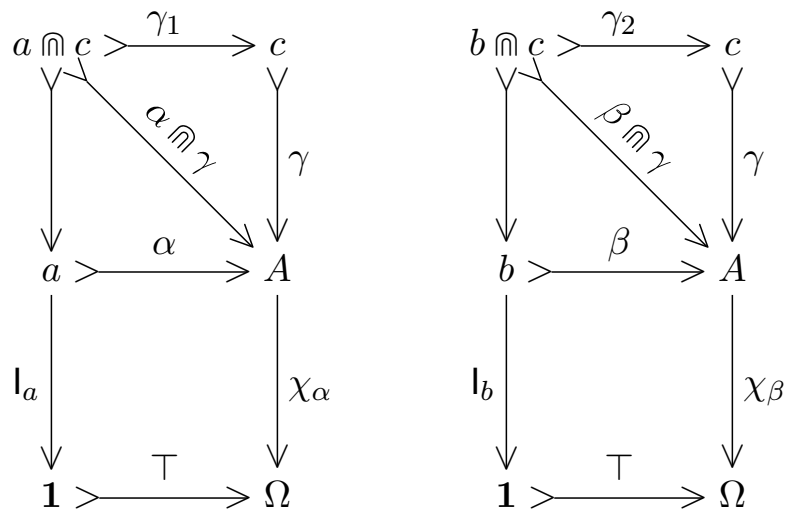

Os quadrados inferiores de ambos são pullbacks pela definição das funções características, enquanto o fato dos quadrados superiores serem pullbacks decorre do teorema 4.1.4. Pelo lema do pullback, pois, temos que os diagramas
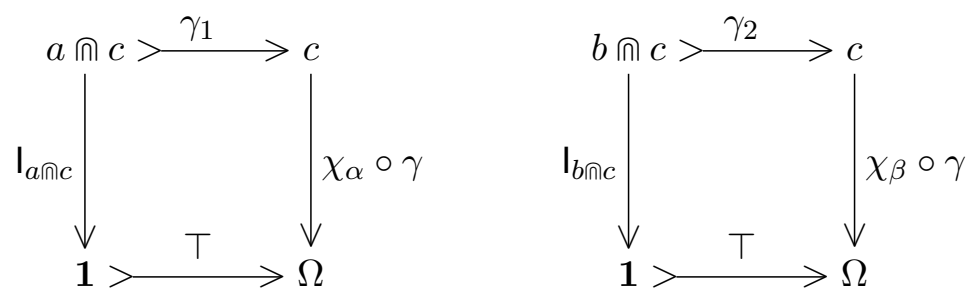

também são pullbacks. Logo, $\chi_{\alpha} \circ \gamma=\chi_{\gamma_{1}}$ e $\chi_{\beta} \circ \gamma=\chi_{\gamma_{2}}$, por causa da unicidade na definição do classificador de subobjeto.

Com isso, $\alpha \cap \gamma \sim \beta \cap \gamma \Leftrightarrow$ existe $\kappa$ iso tal que $(\alpha \cap \gamma) \circ \kappa=\beta \cap \gamma$, isto é, $\gamma \circ \gamma_{1} \circ \kappa=\gamma \circ \gamma_{2}$. Como $\gamma$ é mono, isso ocorre $\Leftrightarrow \gamma_{1} \circ \kappa=\gamma_{2}$, ou seja, $\gamma_{1} \sim \gamma_{2}$. Isso, por sua vez, é equivalente a $\chi_{\gamma_{1}}=\chi_{\alpha} \circ \gamma=\chi_{\beta} \circ \gamma=\chi_{\gamma_{2}}$.

2. Imediata da anterior 
3. Temos:

$$
\begin{aligned}
\alpha \cap \gamma \leq[\beta] & \Leftrightarrow(\alpha \cap \gamma) \cap \beta=\alpha \cap \gamma & \\
& \Leftrightarrow(\alpha \cap \beta) \cap \gamma=\alpha \cap \gamma & \text { (usando que } \operatorname{Sub}(A) \text { é reticulado) } \\
& \Leftrightarrow \chi_{\alpha \cap \beta} \circ \gamma=\chi_{\alpha} \circ \gamma & \text { (pelo primeiro item) }
\end{aligned}
$$

Teorema 4.3.5. Sejam $\alpha: a \longmapsto A, \beta: b \longmapsto A$ e $\gamma: c \longmapsto A$ em $\mathcal{E}_{1}$. Então:

1. $\gamma \preceq(\alpha \Rightarrow \beta)$ se, e somente se, $\alpha \cap \gamma \leq[\beta]$;

2. $\alpha \preceq \beta$ se, e somente se, $(\alpha \Rightarrow \beta) \sim i d_{A}$;

3. $\alpha \preceq \beta$ se, e somente se, $\left(\chi_{\alpha} \mapsto \chi_{\beta}\right)=\top_{A}$.

Demonstração. 1. Primeiramente, da definição de $\alpha \Rightarrow \beta$, temos que $\left(\chi_{\alpha} \Leftrightarrow \chi_{\beta}\right) \circ(\alpha \Rightarrow \beta)=$ $\top \circ \mathbf{I}_{\alpha \Rightarrow \beta}$. Assim, usando o pullback da definição de $\Leftrightarrow$, existe uma única $\iota:(a \Rightarrow b) \rightarrow \preccurlyeq$ que torna o diagrama comutativo:

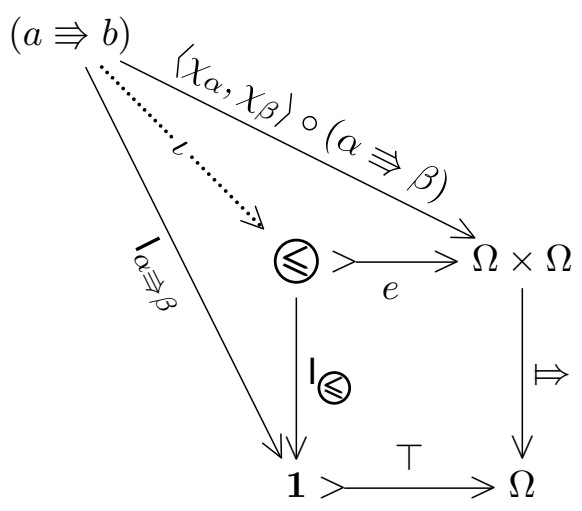

Com isso, consideremos o diagrama:

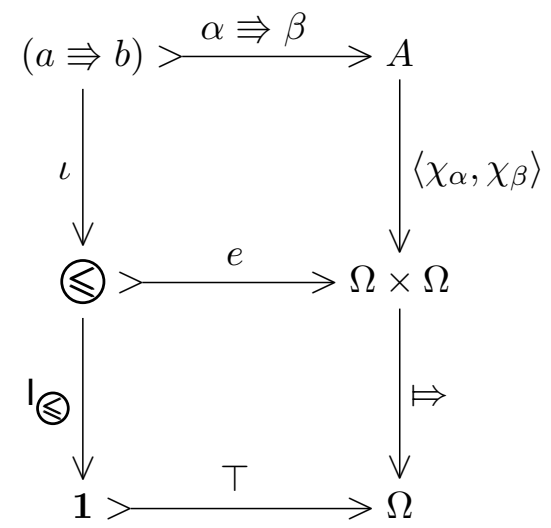


O quadrado inferior é um pullback pela definição de $\Leftrightarrow$, e o retângulo é um pullback pela definição de $\alpha \Rightarrow \beta$. Portanto, pelo lema do pullback, o quadrado superior também é um pullback.

Agora, suponha que $\gamma \preceq(\alpha \Rightarrow \beta)$, isto é, existe $\kappa: c \rightarrow(a \Rightarrow b)$ tal que $(\alpha \Rightarrow \beta) \circ \kappa=\gamma$. Nesse caso, como temos $\left\langle\chi_{a}, \chi_{\beta}\right\rangle \circ(\alpha \Rightarrow \beta)=e \circ \iota$ (pela comutatividade do quadrado de pullback $)$, podemos fazer $\left\langle\chi_{a}, \chi_{\beta}\right\rangle \circ(\alpha \Rightarrow \beta) \circ \kappa=e \circ \iota \circ \kappa$, donde $\left\langle\chi_{a}, \chi_{\beta}\right\rangle \circ \gamma=e \circ(\iota \circ \kappa)$. Com isso, usando que e é o equalizador de $\wedge$ e $\pi_{1}$, temos

$$
\begin{aligned}
\chi_{\alpha} \circ \gamma=\pi_{1} \circ\left\langle\chi_{a}, \chi_{\beta}\right\rangle \circ \gamma=\pi_{1} \circ e \circ(\iota \circ \kappa) & = \\
= & \wedge \circ e \circ(\iota \circ \kappa)=\wedge \circ\left\langle\chi_{a}, \chi_{\beta}\right\rangle \circ \gamma=\chi_{\alpha \circledast \beta} \circ \gamma
\end{aligned}
$$

E, pelo último item do lema anterior, $\chi_{\alpha} \circ \gamma=\chi_{\alpha \circledast \beta} \circ \gamma$ se, e somente se, $\alpha \cap \gamma \leq[\beta]$.

Reciprocamente, suponha que $\alpha \cap \gamma \leq[\beta]$. Usando mais uma vez o lema, isso é equivalente a $\chi_{\alpha} \circ \gamma=\chi_{\alpha \circledast \beta} \circ \gamma$. Assim,

$$
\pi_{1} \circ\left\langle\chi_{a}, \chi_{\beta}\right\rangle \circ \gamma=\chi_{\alpha} \circ \gamma=\chi_{\alpha ® \beta} \circ \gamma=\wedge \circ\left\langle\chi_{a}, \chi_{\beta}\right\rangle \circ \gamma
$$

Como $e$ é o equalizador de $\wedge$ e $\pi_{1}$, existe uma única $\rho: c \rightarrow \S$ tal que $\left\langle\chi_{a}, \chi_{\beta}\right\rangle \circ \gamma=e \circ \rho$. Então, pelo pullback, temos que existe uma única flecha $\xi: c \rightarrow(a \Rightarrow b)$ tal que o diagrama abaixo fica comutativo:

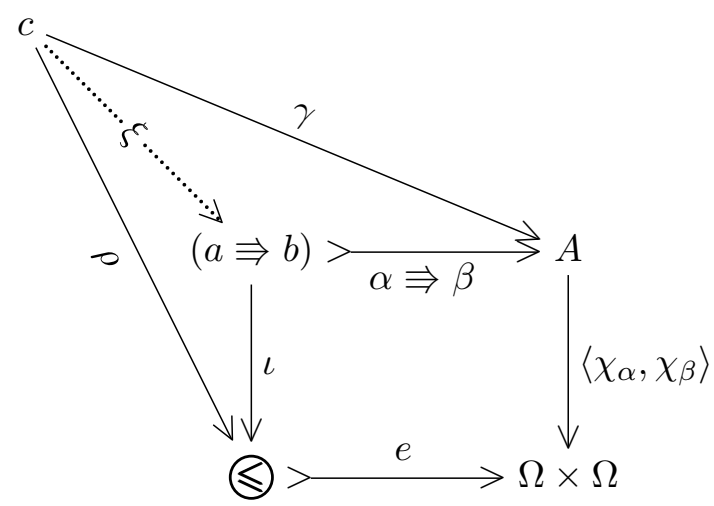

Logo, $(\alpha \Rightarrow \beta) \circ \xi=\gamma$, isto é, $\gamma \preceq(\alpha \Rightarrow \beta)$, como queríamos.

2. Suponha que $\alpha \preceq \beta$. Então, para qualquer $\delta: d \longmapsto A, \alpha \cap \delta \leq[\alpha] \leq[\beta]$. Mas pelo item anterior, isso é equivalente a $\delta \preceq(\alpha \Rightarrow \beta)$. Tomando $\delta=i d_{A}$, como [id $]$ ] é a unidade de $\operatorname{Sub}(a)$, temos $[\alpha \Rightarrow \beta]=\left[i d_{A}\right]$, e $(\alpha \Rightarrow \beta) \sim i d_{A}$.

Agora suponha que $(\alpha \Rightarrow \beta) \sim i d_{A}$. Então, em particular, $\alpha \preceq(\alpha \Rightarrow \beta)$. Novamente usando o item anterior, isso é equivalente a $\alpha \cap \alpha \leq[\beta]$, isto é, $\alpha \preceq \beta$. 
3. Por definição, $\left(\chi_{\alpha} \boxminus \chi_{\beta}\right)=\chi_{(\alpha \equiv \beta)}$, e é fácil ver que o diagrama abaixo é um pullback:

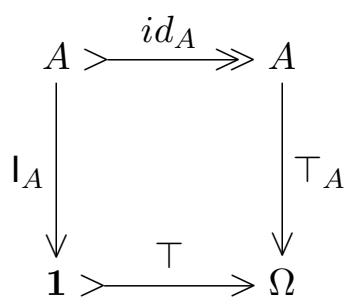

Pela unicidade da flecha característica, $\chi_{i d_{A}}=\top_{A}$. Logo, do item anterior, temos:

$$
\alpha \preceq \beta \quad \Leftrightarrow \quad(\alpha \Rightarrow \beta) \sim i d_{A} \quad \Leftrightarrow \chi_{(\alpha \Rightarrow \beta)}=\chi_{i d_{A}} \quad \Leftrightarrow\left(\chi_{\alpha} \Leftrightarrow \chi_{\beta}\right)=\top_{A}
$$

Com esse teorema, portanto, mostramos que $[\alpha \Rightarrow \beta]$ cumpre o papel da implicação $[\alpha] \rightarrow$ $[\beta]$, e $S u b(A)$ é uma álgebra de Heyting.

\subsection{Negação}

Primeiramente, recordemos que definimos $\perp: \mathbf{1} \rightarrow \Omega$ no capítulo anterior como a flecha característica de $\mathrm{I}_{\mathbf{0}}: \mathbf{0} \rightarrow \mathbf{1}$.

Definição 4.4.1. Definimos a negação $\neg: \Omega \rightarrow \Omega$ como a flecha característica de $\perp: 1 \longmapsto \Omega$ :

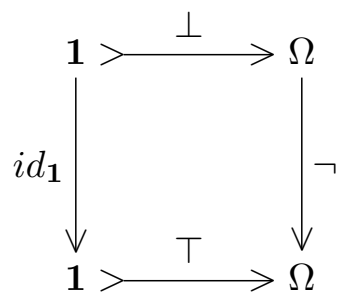

Definição 4.4.2. Dado $\alpha: a \longmapsto A$ em $\mathcal{E}_{1}$, o mono $-\alpha$ é definido como o pullback do mono $\top$ ao longo de $\neg \chi_{\alpha}=\neg 0 \chi_{\alpha}$ :

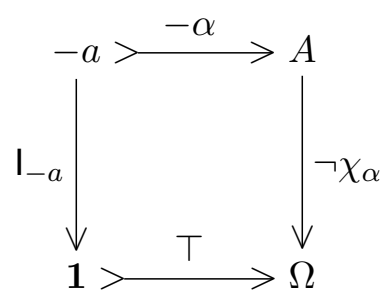

Ou seja, $\neg \chi_{\alpha}=\chi_{-\alpha}$. 
Exemplo 4.4.3. Em Set, $\perp:\{*\} \rightarrow\{0,1\}$ é simplesmente $\perp(*)=0, \log 0 \neg:\{0,1\} \rightarrow\{0,1\}$ é a função tal que $\neg(0)=1$ e $\neg(1)=0$. Assim, dados $a \subseteq A$ e $z \in A,\left(\neg \chi_{a}\right)(z)=1$ se, e só se, $z \notin a$; ou seja, $\neg \chi_{a}$ corresponde à função característica de $(A \backslash a) \subseteq A$.

Teorema 4.4.4. Seja $\alpha: a \rightarrow A$ em $\mathcal{E}_{1}$. Então, $\alpha \cap-\alpha=\left[\mathrm{O}_{A}\right]$.

Demonstração. Considere o diagrama:

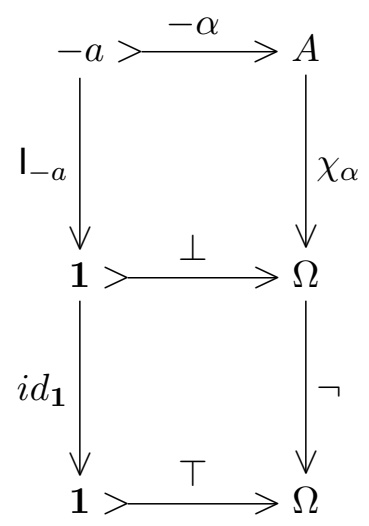

O quadrado inferior é um pullback pela definição de $\neg$, e o retângulo é um pullback pela definição de $-\alpha$. Assim, o lema do pullback garante que o quadrado superior também seja um pullback, e $\chi_{\alpha} \circ-\alpha=\perp \circ \mathrm{I}_{-a}$.

Considere, então, o pullback como no teorema 4.1.4:

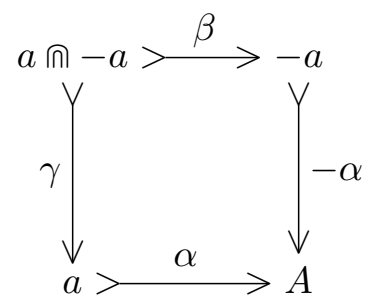

de forma que $-\alpha \circ \beta=\alpha \circ \gamma$.

Portanto, $\chi_{\alpha} \circ-\alpha \circ \beta=\perp \circ \mathbf{I}_{-a} \circ \beta$, e, usando que 1 é o objeto terminal, $\chi_{\alpha} \circ \alpha \circ \gamma=\perp \circ \mathbf{I}_{a \text { ก }-a}$. Mas, pela definição da flecha característica, $\chi_{\alpha} \circ \alpha=\top_{a}$, logo $\chi_{\alpha} \circ \alpha \circ \gamma=T_{a} \circ \gamma$. Novamente usando que 1 é terminal, obtemos:

$$
\perp \circ \mathbf{I}_{a \cap-a}=\chi_{\alpha} \circ \alpha \circ \gamma=T_{a} \circ \gamma=\top \circ \mathbf{I}_{a} \circ \gamma=\top \circ \mathbf{I}_{a 円-a}
$$

Assim, chegamos à igualdade $\perp \circ \mathrm{I}_{a \mathrm{\cap}-a}=\top \circ \mathrm{I}_{a \mathrm{\cap}-a}$, e podemos usar a flecha $\mathrm{I}_{a \mathrm{\cap}-a}$ no da definição de $\perp$ para obter uma flecha única $\kappa: a$ ก $-a \rightarrow \mathbf{0}$ que torna o diagrama a seguir 
comutativo:

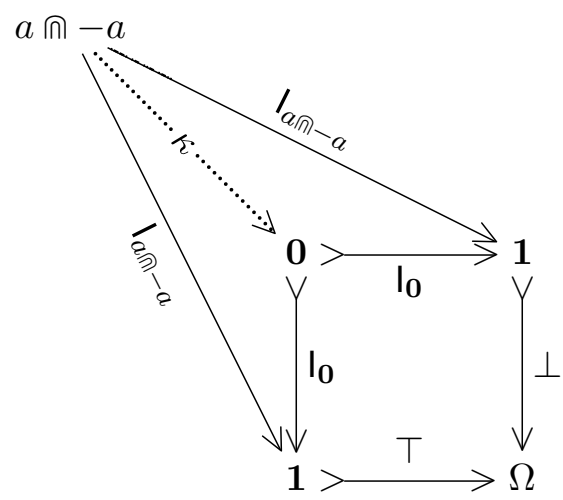

Como 0 é objeto inicial estrito (ver 3.1.4), o fato de existir $\kappa: a$ ก $-a \rightarrow \mathbf{0}$ implica em ( $a$ ก $-a) \cong \mathbf{0}$, e por unicidade $\mathrm{O}_{a \cap-a}: \mathbf{0} \rightarrow(a \cap-a)$ é iso. Além disso, novamente por unicidade, $\alpha$ ก $-\alpha \circ \mathrm{O}_{a \mathrm{\cap}-a}=\mathrm{O}_{A}, \operatorname{logo}[\alpha \cap-\alpha]=\alpha \cap-\alpha=\left[\mathrm{O}_{A}\right]$, como queríamos.

Proposição 4.4.5. Seja $\alpha: a \longmapsto A$ em $\mathcal{E}_{1}$. Então, $[-\alpha]$ é o pseudo-complemento de $\alpha$ em $\operatorname{Sub}(A)$.

Demonstração. Para tando, precisamos mostrar que $-\alpha=\left(\alpha \Rightarrow \mathrm{O}_{A}\right)$. Pelo teorema 4.3.5, temos que, para todo $\beta: b \longmapsto A, \beta \preceq\left(\alpha \Rightarrow \mathrm{O}_{A}\right)$ se, e somente se, $\alpha \cap \beta \leq\left[\mathrm{O}_{A}\right]$. Ou seja, precisamos mostrar que $\beta \preceq-\alpha$ se, e somente se, $\alpha \cap \beta=\left[\mathrm{O}_{A}\right]$ (já usando que $\left[\mathrm{O}_{A}\right]$ é o menor elemento).

Se $[\beta] \leq[-\alpha]$, então, usando que $S u b(A)$ é um reticulado, $\alpha \cap \beta \leq \alpha \cap-\alpha$, e pelo teorema anterior $\alpha \cap-\alpha=\left[\mathrm{O}_{A}\right]$.

Reciprocamente, suponha que $\alpha \cap \beta=\left[\mathrm{O}_{A}\right]$. Então, pelo teorema 4.1.4, o quadrado superior abaixo é um pullback; enquanto o quadrado inferior é um pullback pela definição da flecha característica:

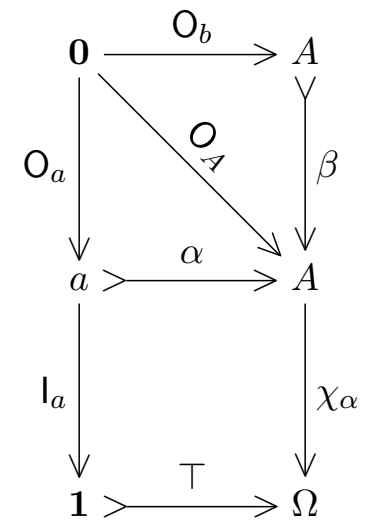

Logo, pelo lema do pullback o retângulo também é um pullback, e temos $\chi_{\alpha} \circ \beta=\chi_{\mathrm{O}_{b}}$. 
Além disso, no diagrama a seguir, o quadrado inferior é um pullback pela definição de $\perp$, e o quadrado superior é um pullback pois objetos iniciais são estritos (então se existem $f, g$ tais que $\mathrm{I}_{\mathbf{0}} \circ g=\mathrm{I}_{b} \circ f$, existe uma flecha $g: x \rightarrow \mathbf{0}$, e $\left.x \cong \mathbf{0}\right)$. Assim, o retângulo também será um pullback, $\mathrm{e} \perp \circ \mathrm{I}_{b}=\chi_{\mathrm{O}_{b}}$ :

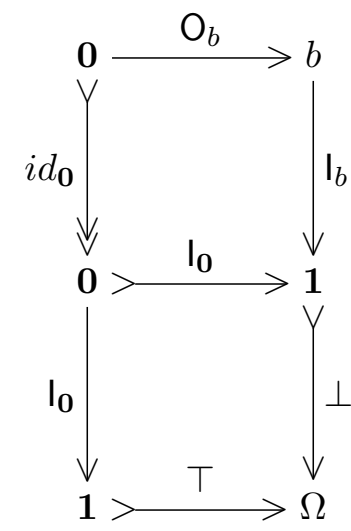

Com isso, vale $\chi_{\alpha} \circ \beta=\chi_{\mathrm{O}_{b}}=\perp \circ \mathrm{I}_{b}$; portanto, usando as definições de $\neg$ e $\chi_{\beta}$, e que $\neg \chi_{\alpha}=\chi_{-\alpha}$, obtemos:

$$
\chi_{-\alpha} \circ \beta=\neg \chi_{\alpha} \circ \beta=\neg \perp \circ \mathbf{I}_{b}=\top \circ \mathbf{I}_{b}=\chi_{\beta} \circ \beta
$$

De forma que, pelo lema 4.3.4, $-\alpha \cap \beta=\beta \cap \beta$. Por fim, $\operatorname{como} S u b(A)$ é reticulado, $[\beta]=\beta \cap \beta=$ $-\alpha \cap \beta \leq[-\alpha]$.

Proposição 4.4.6. $[-\top]=[\perp]$.

Demonstração. Isso decorre facilmente das definições, e usando que $[-T]=[\perp] \Leftrightarrow \chi_{(-\top)}=\chi_{\perp}$ :

$$
\chi_{\perp}=\neg=\neg \circ i d_{\Omega}=\neg \circ \chi_{\top}=\chi_{(-\top)}
$$

Para finalizar essa parte dos conectivos, um breve comentário sobre uma forma indireta de mostrar a conexão: dada uma operação definida em $\Omega \times \Omega \rightarrow \Omega$, temos a correspondência pelo Lema de Yoneda:

$$
\Omega \times \Omega \rightarrow \Omega \quad \leadsto \quad \mathcal{E}(-, \Omega) \times \mathcal{E}(-, \Omega) \rightarrow \mathcal{E}(-, \Omega)
$$

Assim, para cada $A \in \mathcal{E}_{0}$, teríamos uma operação $\mathcal{E}(A, \Omega) \times \mathcal{E}(A, \Omega) \rightarrow \mathcal{E}(A, \Omega)$. Mas já vimos no teorema 3.2.5 que temos a bijeção nautal $\mathcal{E}(A, \Omega) \cong S u b(A)$. Ou seja, isso corresponde a definir 
as operações em $\operatorname{Sub}(A)$.

\subsection{Quantificadores}

Para os quantificadores, usamos fortemente a proposição 3.4.5.

Proposição 4.5.1. Sejam $\mathcal{E}$ um topos e $f: a \rightarrow b$ em $\mathcal{E}_{1}$. Então, o funtor $f^{\star}: \mathcal{E} \downarrow b \rightarrow \mathcal{E} \downarrow a$ restringe-se a um funtor nos subobjetos $f_{\mid}^{\star}:(S u b(b), \leq) \rightarrow(S u b(a), \leq)$, e esse funtor possui adjuntos $\exists_{f} \dashv f_{\mid}^{\star} \dashv \forall_{f}$.

Demonstração. Primeiramente, note que como monos $g: c \longmapsto b$ em $\mathcal{E}_{1}$ correspondem a monos $(c, g)$ em $(\mathcal{E} \downarrow b)_{0}$ e $\mathbf{1}_{b}=\left(b, i d_{b}\right)$ é objeto terminal nessa categoria, podemos definir um isomorfismo entre $\operatorname{Sub}(b)$ e $\operatorname{Sub}\left(\mathbf{1}_{b}\right)$ fazendo $g \mapsto \mathrm{I}_{(c, g)}:(c, g) \rightarrow\left(b, i d_{b}\right)$. Além disso, $\operatorname{Sub}\left(\mathbf{1}_{b}\right)$ é equivalente a $M o n o(b)$, que é subcategoria plena de $\mathcal{E} \downarrow b$ (pois se $\alpha:(c, f) \rightarrow\left(c^{\prime}, f^{\prime}\right)$ é uma flecha em $\mathcal{E} \downarrow b$ com $f$ e $f^{\prime}$ monos, então $f^{\prime} \circ \alpha=f$, donde $\alpha$ é mono). Assim, a ideia será usar a adjunção $\Sigma_{f} \dashv f^{\star} \dashv \Pi_{f}$ para obter $\exists_{f} \dashv f_{\mid}^{\star} \dashv \forall_{f}$ :

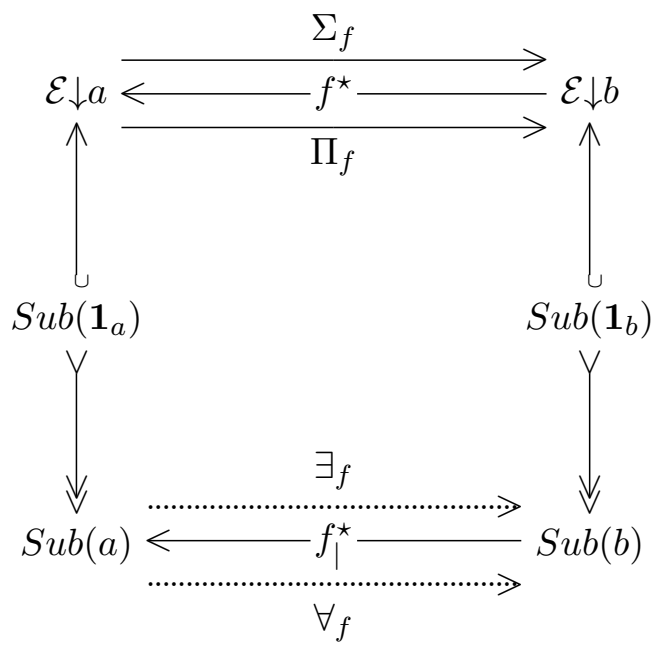

Como $f^{\star}$ e $\Pi_{f}$ possuem adjuntos à esquerda, ambos preservam objeto terminal e pullbacks, logo também monos (pois todo mono pode ser obtido do kernel par, proposição 1.5.8). Dessa forma, já fazendo a identificação $S u b(b) \cong S u b\left(\mathbf{1}_{b}\right)$, ambos restringem-se a funtores $f_{\mid}^{\star}:(\operatorname{Sub}(b), \leq) \rightarrow(\operatorname{Sub}(a), \leq)$ e $\forall_{f}:(\operatorname{Sub}(a), \leq) \rightarrow(S u b(b), \leq)$, e a adjunção $f^{\star} \dashv \Pi_{f}$ induz uma adjunção $f_{\mid}^{\star} \dashv \forall_{f}{ }^{1}$

O adjunto à esquerda $\exists_{f}:(S u b(a), \leq) \rightarrow(S u b(b), \leq)$ também pode ser obtido usando $\Sigma_{f}$,

\footnotetext{
${ }^{1}$ Mais precisamente, a adjunção ser induzida para os subobjetos decorre de um argumento semelhante àquele da existência de $\Pi_{f}$ usando que o funtor partes é monádico.
} 
mas com pequenas alterações pois monos não são preservados. Assim, para cada $[h] \in S u b(a)$, tome $\exists_{f}([h])=[i m(f \circ h)]$, e é fácil verificar que está bem definido por causa da unicidade a menos de isomorfismo da fatoração epi-mono.

Agora, verifiquemos a adjunção, lembrando o exemplo 1.7.3 de adjunções em conjuntos parcialmente ordenados. Sejam $g: c \longmapsto b$ e $h: d \longmapsto a$ em $\mathcal{E}_{1}$. Se $[h] \leq f_{\mid}^{\star}([g])$, então, chamando de $\left(p, f_{g}^{\prime}, g^{\prime}\right)$ o pullback de $f$ e $g$, existe $k: d \rightarrow c$ tal que $g^{\prime} \circ k=h$. Assim, $f \circ g^{\prime} \circ k=g \circ f_{g}^{\prime} \circ k=f \circ h$, e $f \circ h$ se fatora por um mono $g$; logo, pela demonstração do teorema 3.4.8, $\exists_{f}(h)=[i m(f \circ h)] \leq[g]$. Reversamente, se $\exists_{f}(h) \leq[g]$, então existe $t:(f \circ h)(d) \rightarrow b$ tal que $g \circ t=i m(f \circ h)$. Com isso, $f \circ h=i m(f \circ h) \circ(f \circ h)^{\triangleright}=g \circ t \circ(f \circ h)^{\triangleright}$, e usando o pullback de $f$ e $g$, existe única $\kappa: d \rightarrow p$ tornando o diagrama comutativo:

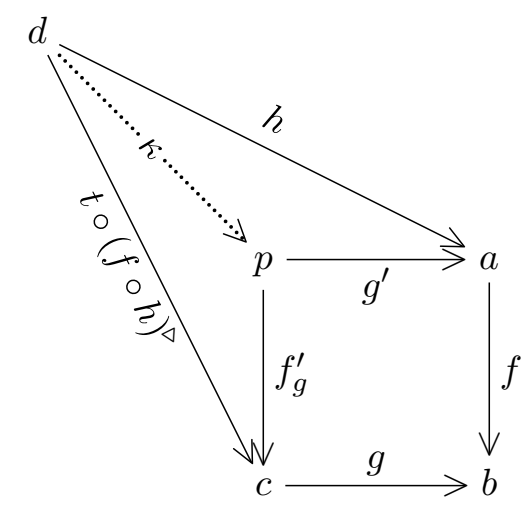

donde $h=g^{\prime} \circ \kappa$ e $[h] \leq\left[g^{\prime}\right]=f_{\mid}^{\star}([g])$.

No futuro, quando não houver risco de ambiguidade, será comum denotar a "restrição" $f_{\mid}^{\star}$ simplesmente por $f^{\star}$ (para simplificar a notação).

Para deixar mais clara a motivação para os quantificadores serem definidos dessa forma, façamos rapidamente como os quantificadores atuam em Set.

Exemplo 4.5.2. Seja $f: A \rightarrow B$ uma função em Set $_{1}$. Dado um subconjunto $S \subseteq A$, identifiquemo-lo com sua inclusão $i_{S}: S \hookrightarrow A$ (para facilitar a escrita), de forma que:

$$
\exists_{f}(S)=f(S)=\{f(a) \mid a \in S\}=\{b \in B \mid \exists a \in S(f(a)=b)\}
$$

Agora, para o quantificador universal, vejamos as categorias slice em Set como conjuntos indexados (exemplo 1.8.4). Assim, dada uma função $f: A \rightarrow B$, o funtor $f^{\star}: \operatorname{Set} \downarrow B \rightarrow \operatorname{Set} \downarrow A$ será dado por $f^{\star}\left(\left\{Y_{b} \mid b \in B\right\}\right)=\left\{Y_{f(a)} \mid a \in A\right\}$. Com isso, pode-se verificar que o adjunto à 
esquerda desse funtor será dado por:

$$
\Sigma_{f}\left(\left\{X_{a} \mid a \in A\right\}\right)=\left\{\coprod_{f(a)=b} X_{a} \mid b \in B\right\}
$$

e o adjunto à direita será dado por:

$$
\Pi_{f}\left(\left\{X_{a} \mid a \in A\right\}\right)=\left\{\prod_{f(a)=b} X_{a} \mid b \in B\right\}
$$

para toda família $\left\{X_{a} \mid a \in A\right\}^{2}$. Assim, dado um subconjunto $S \subseteq B$, definimos a família $X_{S}=\left\{X_{a} \mid a \in A\right\}$ tomando $X_{a}=\{a\}$ se $a \in S$ e $X_{a}=\emptyset$ caso contrário. Dessa forma, o conjunto $\prod_{f(a)=b} X_{a}$ será unitário se todos os $X_{a}$ forem unitários (e isso ocorre quando $a \in S$ para todo $a \in A$ tal que $f(a)=b$ ) e vazio se pelo menos um deles for vazio. Como subobjeto de $B$, isso significa:

$$
\forall_{f}(S)=\{b \in B \mid \forall a \in A(f(a)=b \Rightarrow a \in S)\}
$$

Para o caso especial que será importante mais à frente em que $f$ é a projeção $\pi_{2}: A \times B \rightarrow B$, dado um subconjunto $S \subseteq A \times B$, obtemos:

$$
\begin{aligned}
& \exists_{\pi_{2}}(S)=\{b \in B \mid \exists a \in A((a, b) \in S)\} \\
& \forall_{\pi_{2}}(S)=\{b \in B \mid \forall a \in A((a, b) \in S)\}
\end{aligned}
$$

\subsection{Linguagem de Mitchell-Bénabou}

Para podermos agora trabalhar com a lógica, precisamos primeiramente definir uma linguagem na qual poderemos estabelecer as noções de fórmulas e validade. A linguagem de MitchellBénabou cumpre exatamente esse papel para a lógica interna do topos.

Definição 4.6.1. A linguagem de Mitchell-Bénabou $\mathcal{L}$ consiste de:

- uma classe de tipos. Essa classe inclui (mas não está necessariamente limitada) aos tipos básicos 1 e $\Omega$, e se $A, B$ são tipos, $A \times B$ e $\Omega^{A}$ também são tipos;

- uma classe de termos, definidos indutivamente. Cada termo $t$ tem um tipo $A$, e denotamos $t: A$. Uma fórmula é um termo de tipo $\Omega$;

\footnotetext{
${ }^{2}$ Observe, aliás, como isso justifica a notação de $\Sigma_{f}$ e $\Pi_{f}$ para tais funtores: quando $A$ é objeto terminal, levam uma família indexada exatamente à sua união disjunta e ao seu produto, respectivamente.
} 
- uma classe de símbolos formais:

- para cada tipo $A$, um conjunto enumerável de variáveis de tipo $A$. Como para termos, se $x$ é uma variável de tipo $A$, denotamos $x: A$;

- para cada tipo $A$, uma classe de constantes de tipo $A$. Mais uma vez, se $\bar{a}$ é uma constante de tipo $A$, denotamos $\bar{a}: A$;

- para tipos $A, B$, uma classe de transformações de $A$ em $B$. Denotamos uma transformação $f$ por $f: A \rightarrow B$.

Com isso, os termos são construídos de forma indutiva:

(C1) se $x: A$ é uma variável, então $x: A$ é um termo com variável livre $x: A$;

(C2) se $t: A$ é um termo com variáveis livres $x_{1}: X_{1}, \ldots, x_{n}: X_{n}$, então a expressão formal $t_{\left(x_{1}, \ldots, x_{n+m}\right)}: A$ é um termo com variáveis livres $x_{1}: X_{1}, \ldots, x_{n}: X_{n}, x_{n+1}$ : $X_{n+1}, \ldots, x_{n+m}: X_{n+m}$;

(C3) se $\bar{a}: A$ é uma constante, então $\bar{a}: A$ é um termo sem variáveis livres;

(C4) se $t: A$ é um termo com variáveis livres $x_{1}: X_{1}, \ldots, x_{n}: X_{n}$ e $f: A \rightarrow B$ é uma transformação de $A$ em $B$, então a expressão formal $f(t): B$ é um termo com variáveis livres $x_{1}: X_{1}, \ldots, x_{n}: X_{n}$;

(C5) se $t: A$ e $s: A$ são termos com variáveis livres $x_{1}: X_{1}, \ldots, x_{n}: X_{n}$, então a expressão formal $(t, s): A \times B$ é um termo com variáveis livres $x_{1}: X_{1}, \ldots, x_{n}: X_{n}$;

(C6) se $t: A$ é um termo com variáveis livres $x_{1}: X_{1}, \ldots, x_{n}: X_{n}$ e, para cada $i \in\{1, \ldots, n\}$, $s_{i}: X_{i}$ é um termo com variáveis livres $y_{1}: Y_{1}, \ldots, y_{m}: Y_{m}$, então a expressão formal $t\left(s_{1}, \ldots, s_{n}\right): A$ é um termo com variáveis livres $y_{1}: Y_{1}, \ldots, y_{m}: Y_{m}$;

(C7) se $\varphi: \Omega$ é uma fórmula com variáveis livres $x_{1}: X_{1}, \ldots, x_{n}: X_{n}, y_{1}: Y_{1}, \ldots, y_{m}: Y_{m}$ (com $x_{i}$ distintos de $y_{j}$, para todos $i, j$ ), então a expressão formal $\left\{x_{1}, \ldots, x_{n} \mid \varphi\right\}$ : $\Omega^{X_{1} \times \ldots \times X_{n}}$ é um termo com variáveis livres $y_{1}: Y_{1}, \ldots, y_{m}: Y_{m}$. Nesse caso, dizemos que $x_{1}: X_{1}, \ldots, x_{n}: X_{n}$ são variáveis ligadas de $\left\{x_{1}, \ldots, x_{n} \mid \varphi\right\}$

(C8) se $t: A$ e $s: A$ são termos com variáveis livres $x_{1}: X_{1}, \ldots, x_{n}: X_{n}$, então a expressão formal $t=s: \Omega$ é uma fórmula com variáveis livres $x_{1}: X_{1}, \ldots, x_{n}: X_{n}$;

(C9) se $t: A$ e $\Sigma: \Omega^{A}$ são termos com variáveis livres $x_{1}: X_{1}, \ldots, x_{n}: X_{n}$, então a expressão formal $t \in \Sigma: \Omega$ é uma fórmula com variáveis livres $x_{1}: X_{1}, \ldots, x_{n}: X_{n}$; 
(C10) se $\varphi: \Omega$ é uma fórmula com variáveis livres $x_{1}: X_{1}, \ldots, x_{n}: X_{n}$, então a expressão formal $\neg \varphi: \Omega$ é uma fórmula com variáveis livres $x_{1}: X_{1}, \ldots, x_{n}: X_{n}$;

(C11) se $\varphi: \Omega$ e $\psi: \Omega$ são fórmulas com variáveis livres $x_{1}: X_{1}, \ldots, x_{n}: X_{n}$, então as expressões formais $\varphi \vee \psi: \Omega, \varphi \wedge \psi: \Omega, \varphi \Rightarrow \psi: \Omega$ são fórmulas com variáveis livres $x_{1}: X_{1}, \ldots, x_{n}: X_{n}$

(C12) se $\varphi: \Omega$ é uma fórmula com variáveis livres $x: X, y_{1}: Y_{1}, \ldots, y_{n}: Y_{n}\left(\operatorname{com} x \neq y_{i}\right.$, para todo $i \in\{1, \ldots, n\})$, então as expressões formais $\exists x \varphi: \Omega$ e $\forall x \varphi: \Omega$ são fórmulas com variáveis livres $y_{1}: Y_{1}, \ldots, y_{n}: Y_{n}$. Nesse caso, dizemos que $x: X$ é uma variável ligada de $\exists x \varphi$ e de $\forall x \varphi$.

Definição 4.6.2. Seja $\mathcal{E}$ um topos. A linguagem de Mitchell-Bénabou $\mathcal{L}_{\mathcal{E}}$ do topos $\mathcal{E}$ consiste em especificar:

- para cada tipo $A$ de $\mathcal{L}$ um objeto $A \in \mathcal{E}_{0}$. Para os tipos 1 e $\Omega$, escolhemos um dos objetos terminais e um dos classificadores de subobjeto, respectivamente;

- para cada termo $t: A$ com variáveis livres $x_{1}: X_{1}, \ldots, x_{n}: X_{n}$ em $\mathcal{L}$, uma flecha $\ulcorner t\urcorner$ : $X_{1} \times \ldots \times X_{n} \rightarrow A$ em $\mathcal{E}_{1}$, chamada de interpretação de $t$

- para cada constante $\bar{a}: A$, uma flecha $a: \mathbf{1} \rightarrow A$;

- para cada transformação $f: A \rightarrow B$ em $\mathcal{L}$, uma flecha $f: A \rightarrow B$ em $\mathcal{E}_{1}$.

A interpretação dos termos é, mais uma vez, definida indutivamente, seguindo as regras de construção de termos:

(C1) se $x: A$ é uma varíavel, então $\ulcorner x\urcorner:=i d_{A}: A \rightarrow A$;

(C2) se $t: A$ é um termo com variáveis livres $x_{1}: X_{1}, \ldots, x_{n}: X_{n}$, então $\left\ulcorner t_{\left(x_{1}, \ldots, x_{n+m}\right)}\right\urcorner:=$ $\ulcorner t\urcorner \circ \pi: X_{1} \times \ldots \times X_{n+m} \rightarrow A$, onde $\pi: X_{1} \times \ldots \times X_{n+m} \rightarrow X_{1} \times \ldots \times X_{n}$ é a projeção nas $n$ primeiras coordenadas (ou seja, pensamos em $t_{\left(x_{1}, \ldots, x_{n+m}\right)}$ como o termo $t$ mas com variáveis $\left.x_{1}, \ldots, x_{n+m}\right)^{3}$;

(C3) se $\bar{a}: A$ é uma constante, então $\ulcorner\bar{a}\urcorner:=a: \mathbf{1} \rightarrow A$;

(C4) se $t: A$ é um termo com variáveis livres $x_{1}: X_{1}, \ldots, x_{n}: X_{n}$ e $f: A \rightarrow B$ é uma flecha em $\mathcal{E}_{1}$, então $\ulcorner f(t)\urcorner:=f \circ\ulcorner t\urcorner: X_{1} \times \ldots \times X_{n} \rightarrow B ;$

\footnotetext{
${ }^{3}$ Por motivos técnicos (para que os domínios das flechas sejam compatíveis), em alguns momentos teremos que fazer a "expansão vazia de variáveis", isto é, consideraremos o termo $t_{(\text {) }}$ com interpretação $\left.\left\ulcorner t_{(}\right)\right\urcorner=\ulcorner t\urcorner \circ \pi_{\bar{X}}$ : $1 \times X_{1} \times \ldots \times X_{n} \rightarrow A$, para $\pi_{\bar{X}}: \mathbf{1} \times X_{1} \times \ldots \times X_{n} \rightarrow X_{1} \times \ldots \times X_{n}$ a projeção nas $n$ últimas coordenadas; o que não é um problema pois tal projeção é um iso (aliás, mais adiante veremos que basta que a projeção seja um epi para que a validade da fórmula seja preservada).
} 
(C5) se $t: A$ e $s: A$ são termos com variáveis livres $x_{1}: X_{1}, \ldots, x_{n}: X_{n}$, então $\ulcorner(t, s)\urcorner:=$ $\langle\ulcorner t\urcorner,\ulcorner s\urcorner\rangle: X_{1} \times \ldots \times X_{n} \rightarrow A \times B ;$

(C6) se $t: A$ é um termo com variáveis livres $x_{1}: X_{1}, \ldots, x_{n}: X_{n}$ e, para cada $i \in\{1, \ldots, n\}$, $s_{i}: X_{i}$ é um termo com variáveis livres $y_{1}: Y_{1}, \ldots, y_{m}: Y_{m}$, então $\left\ulcorner t\left(s_{1}, \ldots, s_{n}\right)\right\urcorner:=$ $\ulcorner t\urcorner \circ\left\langle\left\ulcorner s_{1}\right\urcorner, \ldots,\left\ulcorner s_{n}\right\urcorner\right\rangle: Y_{1} \times \ldots \times Y_{m} \rightarrow A ;$

(C7) se $\varphi: \Omega$ é uma fórmula com variáveis livres $x_{1}: X_{1}, \ldots, x_{n}: X_{n}, y_{1}: Y_{1}, \ldots, y_{m}: Y_{m}$ (com $x_{i}$ distintos de $y_{j}$, para todos $i, j$ ), então $\left\ulcorner\left\{x_{1}, \ldots, x_{n} \mid \varphi\right\}\right\urcorner:=\exp (\ulcorner\varphi\urcorner)$ : $Y_{1} \times \ldots \times Y_{m} \rightarrow \Omega^{X_{1} \times \ldots \times X_{n}}$

(C8) se $t: A$ e $s: A$ são termos com variáveis livres $x_{1}: X_{1}, \ldots, x_{n}: X_{n}$, então $\ulcorner t=$ $s\urcorner:=\ominus_{A} \circ\langle\ulcorner t\urcorner,\ulcorner s\urcorner\rangle: X_{1} \times \ldots \times X_{n} \rightarrow \Omega$, onde $\ominus_{A}:=\chi_{\left\langle i d_{A}, i d_{A}\right\rangle}: A \times A \rightarrow \Omega(\mathrm{a}$ característica da diagonal $\left.\Delta_{A}:=\left\langle i d_{A}, i d_{A}\right\rangle: A \longmapsto A \times A\right)$;

(C9) se $t: A$ e $\Sigma: \Omega^{A}$ são termos com variáveis livres $x_{1}: X_{1}, \ldots, x_{n}: X_{n}$, então $\ulcorner t \in$ $\Sigma\urcorner:=e v_{\Omega} \circ\langle\ulcorner\Sigma\urcorner,\ulcorner t\urcorner\rangle: X_{1} \times \ldots \times X_{n} \rightarrow \Omega ;$

(C10) se $\varphi: \Omega$ é uma fórmula com variáveis livres $x_{1}: X_{1}, \ldots, x_{n}: X_{n}$, então $\ulcorner\neg \varphi\urcorner:=$ $\neg \circ\ulcorner\varphi\urcorner: X_{1} \times \ldots \times X_{n} \rightarrow \Omega ;$

(C11) se $\varphi: \Omega$ e $\psi: \Omega$ são fórmulas com variáveis livres $x_{1}: X_{1}, \ldots, x_{n}: X_{n}$, então:

- $\ulcorner\varphi \vee \psi\urcorner:=\vee \circ\langle\ulcorner\varphi\urcorner,\ulcorner\psi\urcorner\rangle: X_{1} \times \ldots \times X_{n} \rightarrow \Omega$;

- $\ulcorner\varphi \wedge \psi\urcorner:=\wedge \circ\langle\ulcorner\varphi\urcorner,\ulcorner\psi\urcorner\rangle: X_{1} \times \ldots \times X_{n} \rightarrow \Omega$;

- $\ulcorner\varphi \Rightarrow \psi\urcorner:=\Leftrightarrow \circ\langle\ulcorner\varphi\urcorner,\ulcorner\psi\urcorner\rangle: X_{1} \times \ldots \times X_{n} \rightarrow \Omega$;

(C12) se $\varphi: \Omega$ é uma fórmula com variáveis livres $x: X, y_{1}: Y_{1}, \ldots, y_{n}: Y_{n}\left(\operatorname{com} x \neq y_{i}\right.$, para todo $i \in\{1, \ldots, n\})$ e $m: A \longmapsto X \times Y_{1} \times \ldots \times Y_{n}$ é tal que $\chi_{m}=\ulcorner\varphi\urcorner$, então:

- $\ulcorner\exists x \varphi\urcorner:=\chi_{f}: Y_{1} \times \ldots \times Y_{n} \rightarrow \Omega$, para algum $f \in \exists_{\pi}([m])$;

- $\ulcorner\forall x \varphi\urcorner:=\chi_{g}: Y_{1} \times \ldots \times Y_{n} \rightarrow \Omega$, para algum $g \in \forall_{\pi}([m])$;

onde $\pi: X \times Y_{1} \times \ldots \times Y_{n} \rightarrow Y_{1} \times \ldots \times Y_{n}$ é a projeção nas $n$ últimas coordenadas.

Aqui definimos a validade especificamente para a linguagem $\mathcal{L}_{\mathcal{E}}$, porém é também possível defini-la para linguagens mais gerais; ver seção II.1 de [LS86] para mais detalhes.

Definição 4.6.3. Validade de fórmulas em $\mathcal{L}_{\mathcal{E}}$ : dada uma fórmula $\varphi: \Omega$ na linguagem $\mathcal{L}_{\mathcal{E}}$ com variáveis livres $x_{1}: X_{1}, \ldots, x_{n}: X_{n}$, dizemos que $\varphi$ é satisfeita em $\mathcal{E}$ se $\ulcorner\varphi\urcorner=\top_{X_{1} \times \ldots \times X_{n}}$ : $X_{1} \times \ldots \times X_{n} \rightarrow \Omega$. Nesse caso, denotamos $\mathcal{E} \models \varphi$. 
Antes de enunciarmos alguns teoremas importantes, façamos dois resultados técnicos sobre expansão de variáveis.

Lema 4.6.4. Sejam $\mathcal{E}$ um topos e $\varphi$ uma fórmula em $\mathcal{L}_{\mathcal{E}}$ com variáveis livres $x_{1}: X_{1}, \ldots, x_{n}: X_{n}$. Então:

1. se $\mathcal{E}=\varphi$, então $\mathcal{E}=\varphi_{\left(x_{1}, \ldots, x_{n+m}\right)}$;

2. se $\mathcal{E} \models \varphi_{\left(x_{1}, \ldots, x_{n+m}\right)}$ e a projeção nas $n$ primeiras coordenadas $\pi: X_{1} \times \ldots \times X_{n+m} \rightarrow$ $X_{1} \times \ldots \times X_{n}$ é epi, então $\mathcal{E} \models \varphi$.

Demonstração. 1. Como $\left\ulcorner\varphi_{\left(x_{1}, \ldots, x_{n+m}\right)}\right\urcorner=\ulcorner\varphi\urcorner \circ \pi$ por definição e $\ulcorner\varphi\urcorner=\top_{X_{1} \times \ldots \times X_{n}}=$ $\mathrm{I}_{X_{1} \times \ldots \times X_{n}} \circ \top$ por hipótese, $\left\ulcorner\varphi_{\left(x_{1}, \ldots, x_{n+m}\right)}\right\urcorner=\top_{x_{1}, \ldots, x_{n+m}}$ decorre simplesmente da propriedade do objeto terminal.

2. Se $\ulcorner\varphi\urcorner \circ \pi=\left\ulcorner\varphi_{\left(x_{1}, \ldots, x_{n+m}\right)}\right\urcorner=\top_{x_{1}, \ldots, x_{n+m}}$, então $\pi$ ser a projeção implica em $\ulcorner\varphi\urcorner \circ \pi=$ $\top_{X_{1} \times \ldots \times X_{n}} \circ \pi$. Como supusemos que $\pi$ é epi, obtemos $\ulcorner\varphi\urcorner=\top_{X_{1} \times \ldots \times X_{n}}$.

Proposição 4.6.5. Sejam $\mathcal{E}$ um topos e $\varphi$ uma fórmula em $\mathcal{L}_{\mathcal{E}}$ com variáveis livres $x_{1}$ : $X_{1}, \ldots, x_{n}: X_{n}$. Se para todo $i \in\{1, \ldots, n+m\}$ existe $j \in\{1, \ldots, n\}$ tal que $X_{i}=X_{j}$ (ou seja, se expandirmos a fórmula apenas com variáveis com tipos já presentes na fórmula original), então $\mathcal{E} \models \varphi$ se, e somente se, $\mathcal{E} \models \varphi_{\left(x_{1}, \ldots, x_{n+m}\right)}$.

Demonstração. Para cada $i \in\{n, \ldots, n+m\}$, escolha $\varepsilon(i) \in\{1, \ldots, n\}$ tal que $X_{i}=X_{\varepsilon(i)}$, e para cada $i \in\{1, \ldots, n\}$ coloque $\varepsilon(i)=i$. Consideremos, assim, as projeções $\pi_{\varepsilon(i)}: X_{1} \times \ldots \times X_{n} \rightarrow X_{i}$ para cada $i \in\{1, . ., n+m\}$, e a flecha única $\kappa:=\left\langle\pi_{1}, \ldots, \pi_{n+m}\right\rangle$ obtida do produto:

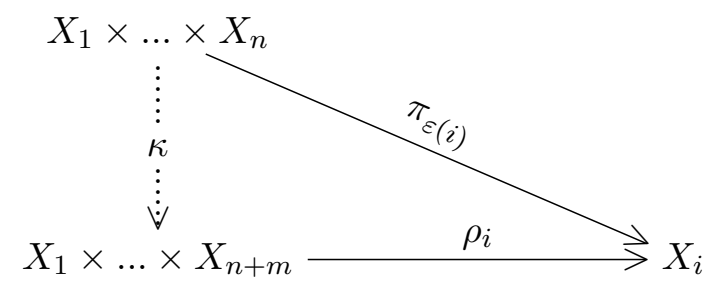

Note que a projeção nas $n$ primeiras coordenadas $\pi: X_{1} \times \ldots \times X_{n+m} \rightarrow X_{1} \times \ldots \times X_{n}$ satisfaz $\pi_{j} \circ \pi=\rho_{j}$, para todo $j \in\{1, \ldots, n\}$, portanto:

$$
\pi_{j} \circ \pi \circ \kappa=\rho_{j} \circ \kappa=\pi_{\varepsilon(j)}=\pi_{j}
$$


donde $\pi \circ \kappa$ é a identidade (pela unicidade da flecha do produto $X_{1} \times \ldots \times X_{n} \rightarrow X_{1} \times \ldots \times X_{n}$ ), e $\pi$ é um epi. Assim, o resultado da proposição segue do lema anterior.

Proposição 4.6.6. Funtores lógicos preservam a validade de fórmulas.

Demonstração. Primeiramente, funtores lógicos preservam a exponenciação, e todas as construções usadas para definir os conectivos são preservadas por funtores lógicos. De fato, como preservam limites e colimites finitos, também preservam monos e epis, logo preservam a fatoração epi-mono. Além disso, flechas características são preservadas, pois funtores lógicos preservam pullbacks, monos e o classificador de subobjeto. Dessa forma, é fácil verificar que funtores lógicos preservam os itens $(\mathbf{C} 1)$ a (C11) da definição indutiva de fórmulas.

Em relação aos quantificadores, o adjunto à esquerda $\exists_{f}$ é definido usando a imagem, logo é preservado por funtores lógicos. Já o adjunto à direita $\forall_{f}$ é a restrição do funtor $\Pi_{f}$, que pode ser obtido usando que o funtor partes é monádico. Tal construção é preservada por funtores lógicos, pois preservam partes e preservam também $f^{\star}$ (que é apenas o pullback) e $\Sigma_{f}$ (que é simplesmente a precomposição), logo $\Pi_{f}$ e $\forall_{f}$ serão igualmente preservados.

No próximo lema, e nos resultados seguintes, dada uma fórmula $\varphi$ com variáveis livres $x_{1}: X_{1}, \ldots, x_{n}: X_{n}$, chamando $\bar{X}=X_{1} \times \ldots \times X_{n}$, denotamos por $\llbracket \varphi \rrbracket \in S u b(\bar{X})$ o subobjeto associado a $\ulcorner\varphi\urcorner: \bar{X} \rightarrow \Omega$ pelo isomorfismo $\operatorname{Sub}(\bar{X}) \cong \mathcal{E}(\bar{X}, \Omega)$ (ver lema 3.2.4). Em particular, $\chi_{m}=\ulcorner\varphi\urcorner$, para todo $m \in \llbracket \varphi \rrbracket$.

Lema 4.6.7. Sejam $\mathcal{E}$ um topos e $\varphi, \psi$ fórmulas em $\mathcal{L}_{\mathcal{E}}$ com variáveis livres $x_{1}: X_{1}, \ldots, x_{n}: X_{n}$. Então, $\mathcal{E} \models \varphi \Rightarrow \psi$ se, e somente se, $\llbracket \varphi \rrbracket \leq \llbracket \psi \rrbracket$.

Demonstração. Pelas definições de interpretação e validade, temos $\ulcorner\varphi \Rightarrow \psi\urcorner=\ulcorner\varphi\urcorner \Leftrightarrow\ulcorner\psi\urcorner=$ $\top_{\bar{X}}$. Mas usando o teorema 4.3 .5 e a definição de $\llbracket \varphi \rrbracket, \llbracket \psi \rrbracket$, isso é equivalente a $\llbracket \varphi \rrbracket \leq \llbracket \psi \rrbracket$.

Teorema 4.6.8. Teorema da correção: $\operatorname{se}_{I} \varphi$ (isto é, se $\varphi$ é um teorema do cálculo de predicados intuicionista), então $\mathcal{E} \models \varphi$, para todo topos $\mathcal{E}$.

Demonstração. A ideia da demonstração é mostrar que os axiomas do cálculo de predicados intuicionista são válidos num topos, depois fazer indução no comprimento da prova do teorema. Ou seja, num topos, dadas $\varphi, \psi, \theta$ fórmulas, $x: X$ uma variável e $t: X$ um termo, vale:

(a) $\mathcal{E} \models \varphi \Rightarrow(\psi \Rightarrow \varphi)$; 
(b) $\mathcal{E}=(\varphi \Rightarrow(\psi \Rightarrow \theta)) \Rightarrow((\varphi \Rightarrow \psi) \Rightarrow(\varphi \Rightarrow \theta))$;

(c) $\mathcal{E}=\varphi \Rightarrow(\psi \Rightarrow(\varphi \wedge \psi))$;

(d) $\mathcal{E} \models(\varphi \wedge \psi) \Rightarrow \varphi$;

(e) $\mathcal{E} \models(\varphi \wedge \psi) \Rightarrow \psi$;

(f) $\mathcal{E}=\varphi \Rightarrow(\varphi \vee \psi)$;

(g) $\mathcal{E}=\psi \Rightarrow(\varphi \vee \psi)$;

(h) $\mathcal{E}=(\varphi \Rightarrow \theta) \Rightarrow((\psi \Rightarrow \theta) \Rightarrow((\varphi \vee \psi) \Rightarrow \theta))$;

(i) $\mathcal{E} \mid=(\varphi \Rightarrow \psi) \Rightarrow((\varphi \Rightarrow \neg \psi) \Rightarrow \neg \varphi)$;

(j) $\mathcal{E} \models \neg \varphi \Rightarrow(\varphi \Rightarrow \psi)$;

(k) Modus ponens: se $\mathcal{E}=\varphi$ e $\mathcal{E}=\varphi \Rightarrow \psi$, então $\mathcal{E}=\psi$;

(l) $\mathcal{E}=(\forall x(\varphi \Rightarrow \psi)) \Rightarrow((\forall x \varphi) \Rightarrow(\forall x \psi))$;

(m) $\mathcal{E} \models(\exists x(\varphi \Rightarrow \psi)) \Rightarrow((\exists x \varphi) \Rightarrow(\exists x \psi)) ;$

(n) se $x$ não é livre em $\varphi$, então $\mathcal{E} \models \varphi \Rightarrow(\forall x \varphi)$;

(o) se $x$ não é livre em $\varphi$, então $\mathcal{E} \models(\exists x \varphi) \Rightarrow \varphi$;

(p) se $t$ não contém nenhuma variável ligada de $\varphi$, então $\mathcal{E} \models(\forall x \varphi) \Rightarrow \varphi(t \mid x)$;

(q) se $t$ não contém nenhuma variável ligada de $\varphi$, então $\mathcal{E} \models \varphi(t \mid x) \Rightarrow(\exists x \varphi)$;

(r) Generalização universal: se $\mathcal{E} \models \varphi$, então $\mathcal{E} \models(\forall x \varphi)$;

onde $\varphi(t \mid x)$ é a fórmula obtida substituindo-se $x$ por $t$ em $\varphi$.

Com pequenas modificações técnicas (referentes principalmente à expansão de variáveis), a satisfação dos axiomas proposicionais (itens (a) a (k) acima) decorre do fato dos subobjetos possuírem uma estrutura de álgebra de Heyting. Apenas modus ponens não é tão imediata, pois tem um aspecto um pouco diferente (sendo uma regra de inferência), mas decorre facilmente do lema acima. Façamos o argumento para modus ponens abaixo, como ilustração.

Suponha que $\mathcal{E}=\varphi$ e $\mathcal{E} \models \varphi \Rightarrow \psi$. É imediato da definição de flecha característica que $\llbracket \top_{\bar{X}} \rrbracket=\left[i d_{\bar{X}}\right]=1$, o maior elemento de $\operatorname{Sub}(\bar{X})$ (para $\llbracket \top_{\bar{X}} \rrbracket$ o subobjeto associado a $\top_{\bar{X}}$ ). Então, as hipóteses significam que $\llbracket \varphi \rrbracket=1 \mathrm{e} \llbracket \varphi \rrbracket \leq \llbracket \psi \rrbracket$, donde $\llbracket \psi \rrbracket=1$. Ou seja, $\mathcal{E} \models \psi$, como desejado. 
Conquanto não tão imediatos quanto os proposicionais, os axiomas com quantificadores decorrem essencialmente da definição dos quantificadores como adjuntos e da estrutura de álgebra de Heyting dos subobjetos (novamente a menos de pequenas questões técnicas envolvendo as variáveis). Como exemplo, façamos o item (l). Sejam $\varphi, \psi$ fórmulas com variáveis livres $x$ : $X, y_{1}: Y_{1}, \ldots, y_{n}: Y_{n}\left(\operatorname{com} x \neq y_{i}\right.$, para todo $\left.i \in\{1, \ldots, n\}\right)$ e $\pi: X \times Y_{1} \times \ldots \times Y_{n} \rightarrow Y_{1} \times \ldots \times Y_{n}$ a projeção nas $n$ últimas coordenadas.

Primeiramente, note que, para todo subobjeto $S, \forall_{\pi}(S) \leq \forall_{\pi}(S)$, portanto $\pi^{\star}\left(\forall_{\pi}(S)\right) \leq S$. Dessa forma, usando também a estrutura de álgebra de Heyting, vale:

$$
\pi^{\star}(\llbracket \forall x(\varphi \Rightarrow \psi) \rrbracket) \wedge \pi^{\star}(\llbracket \forall x \varphi \rrbracket) \leq \llbracket \varphi \Rightarrow \psi \rrbracket \wedge \llbracket \varphi \rrbracket=(\llbracket \varphi \rrbracket \rightarrow \llbracket \psi \rrbracket) \wedge \llbracket \varphi \rrbracket=\llbracket \varphi \rrbracket \wedge \llbracket \psi \rrbracket \leq \llbracket \psi \rrbracket
$$

Mas, por outro lado, temos:

$$
\begin{array}{rr}
\llbracket \forall x(\varphi \Rightarrow \psi) \rrbracket \leq \llbracket \forall x \varphi \Rightarrow \forall x \psi \rrbracket=\llbracket \forall x \varphi \rrbracket \rightarrow \llbracket \forall x \psi \rrbracket & \text { (o que queremos provar) } \\
\Leftrightarrow \llbracket \forall x(\varphi \Rightarrow \psi) \rrbracket \wedge \llbracket \forall x \varphi \rrbracket \leq \llbracket \forall x \psi \rrbracket=\forall \pi(\llbracket \psi \rrbracket) & \text { (propriedade da implicação) } \\
\Leftrightarrow \pi^{\star}(\llbracket \forall x(\varphi \Rightarrow \psi) \rrbracket \wedge \llbracket \forall x \varphi \rrbracket) \leq \llbracket \psi \rrbracket & \text { (pois } \pi^{\star} \dashv \forall_{\pi} \text { ) } \\
\Leftrightarrow \pi^{\star}(\llbracket \forall x(\varphi \Rightarrow \psi) \rrbracket) \wedge \pi^{\star}(\llbracket \forall x \varphi \rrbracket) \leq \llbracket \psi \rrbracket & \text { (pois } \pi^{\star} \text { preserva limites finitos) }
\end{array}
$$

Assim, usando o lema, obtemos o axioma (1).

Demonstrações mais detalhadas e completas podem ser consultadas em [Bor08c] (teoremas 6.7.1 e 6.8.1) ou [Mcl95] (seção 15.2).

Existe também a recíproca do teorema da correção:

Teorema 4.6.9. Teorema da completude: se, para todo topos $\mathcal{E}, \mathcal{E} \models \varphi$ (para $\varphi$ uma fórmula em $\left.\mathcal{L}_{\mathcal{E}}\right)$, então $\vdash_{I} \varphi$.

Essencialmente, a ideia é que, se uma fórmula não é um teorema intuicionista, então é possível construir um topos que não a valida. Em certo sentido, poderíamos compreender esse resultado como "a 'intersecção' das lógicas de todos os topoi é exatamente a lógica de predicados intuicionista".

Apesar da importância conceitual desse teorema, note que a lógica de topos não é completa para cada topos individualmente (por exemplo, num topos booleano a regra do terceiro excluído é satisfeita, mas não é demonstrável na lógica intuicionista); por isso esse resultado é menos usado 
no estudo da lógica de topos. Assim, apenas recomendemos referências para sua demonstração: seção D1.4 de [Joh02b] (mais especificamente o teorema D1.4.11), ou seções II.17 e II.19 de [LS86].

Façamos agora alguns resultados para ilustrar a lógica de topos em funcionamento, começando com como ela pode ser usada para descrever propriedades de flechas numa linguagem mais familiar.

Proposição 4.6.10. Sejam $\mathcal{E}$ um topos, $f, g: a \rightarrow b$ em $\mathcal{E}_{1}$ e $x: A, x^{\prime}: A, y: B$ variáveis. Então:

1. $f=g$ se, e somente se, $\mathcal{E}=\forall x(f(x)=g(x))$;

2. $f$ é um mono se, e somente se, $\mathcal{E} \models \forall x \forall x^{\prime}\left(f(x)=f\left(x^{\prime}\right) \Rightarrow x=x^{\prime}\right)$;

3. $f$ é um epi se, e somente se, $\mathcal{E} \models \forall y \exists x(f(x)=y)$.

Demonstração. Primeiramente, note que se uma variável $x$ não aparece ligada numa fórmula $\varphi$ (como nas fórmulas que aparecem após os quantificadores acima), então podemos substituir a variável $x$ pelo termo $x$ e obter, pelo axioma (p) no teorema 4.6.8, $\mathcal{E} \models \forall x \varphi \Rightarrow \varphi$. Com isso, se $\mathcal{E} \models \forall x \varphi$, por modus ponens obtemos $\mathcal{E} \models \varphi$.

Reciprocamente, se $\mathcal{E} \models \varphi$, por generalização universal (novamente ver teorema 4.6.8), $\mathcal{E} \models \forall x \varphi$. Ou seja, nesse caso mostrar que $\mathcal{E} \models \varphi$ é equivalente a mostrar que $\mathcal{E} \models \forall x \varphi$.

1. Seja $(e, \varepsilon)$ o equalizador de $f$ e $g$. Assim, temos que o diagrama abaixo é um pullback:

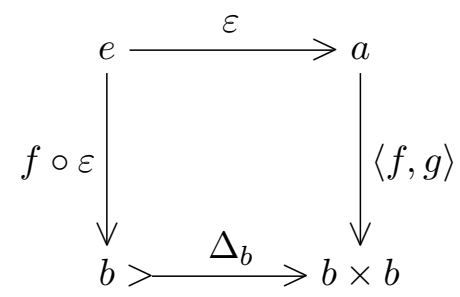

pois, se $\gamma_{1}: c \rightarrow a$ e $\gamma_{2}: c \rightarrow b$ são flechas tais que $\langle f, g\rangle \circ \gamma_{1}=\Delta_{b} \circ \gamma_{2}$, então $\left\langle f \circ \gamma_{1}, g \circ \gamma_{1}\right\rangle=$ $\left\langle\gamma_{2}, \gamma_{2}\right\rangle$, isto é, $f \circ \gamma_{1}=g \circ \gamma_{1}$. Dessa forma, como $(e, \varepsilon)$ é o equalizador, existe uma única $\kappa: c \rightarrow e$ tal que $\varepsilon \circ \kappa=\gamma_{1}$, donde também obtemos $f \circ \varepsilon \circ \kappa=f \circ \gamma_{1}=\gamma_{2}$.

Por outro lado, o quadrado inferior do diagrama abaixo é um pullback pela definição de 
$\bigoplus_{b}$, logo o retângulo também é um pullback:

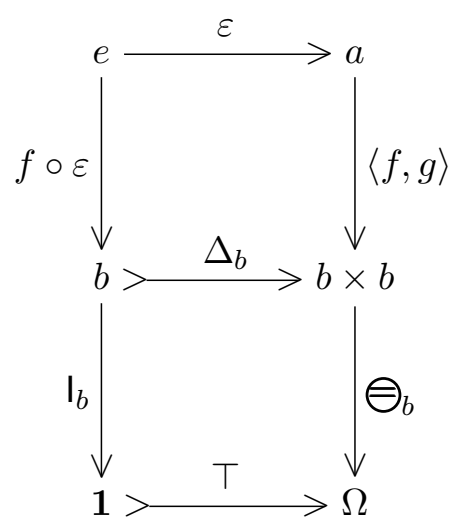

Agora, é imediato da definição de flecha característica que $T_{a}=\chi_{i d_{a}}$. Portanto, como por definição temos $\ulcorner f(x)=g(x)\urcorner=\bigoplus_{b} \circ\left\langle f \circ i d_{a}, g \circ i d_{a}\right\rangle$, pelo pullback acima temos as seguintes equivalências:

$$
\begin{array}{rlrl}
\mathcal{E} F f(x)=g(x) & \Leftrightarrow\ulcorner f(x)=g(x)\urcorner=\top_{a} & \text { (pela definição da validade) } \\
& \Leftrightarrow \chi_{\varepsilon}=\chi_{i d_{a}} & \text { (pelas contas acima) } \\
& \Leftrightarrow \varepsilon \sim i d_{a} & \text { (pela proposição 3.2.3) } \\
& \Leftrightarrow \varepsilon \text { é iso } & \text { (imediato da definição de } \sim \text { ) } \\
& \Leftrightarrow f=g
\end{array}
$$

Essa última decorre do fato de $(e, \varepsilon)$ ser o equalizador: se $f \circ \varepsilon=g \circ \varepsilon$ e $\varepsilon$ é iso, então $f=g$; reciprocamente, se $f=g$, então $\left(a, i d_{a}\right)$ é equalizador. Logo, pelos comentários do início da demonstração, $f=g \Leftrightarrow \mathcal{E}=\forall x(f(x)=g(x))$.

2. Primeiramente, note que tomamos uma certa liberdade de notação no enunciado: a igualdade está definida apenas para termos com mesmas variáveis livres, então na verdade as igualdades são $f(x)_{\left(x, x^{\prime}\right)}=f\left(x^{\prime}\right)_{\left(x, x^{\prime}\right)}$ e $x_{\left(x, x^{\prime}\right)}=x_{\left(x, x^{\prime}\right)}^{\prime}$. Dessa forma, usando a definição de interpretação, obtemos:

$$
\begin{aligned}
& \left\ulcorner f(x)_{\left(x, x^{\prime}\right)}=f\left(x^{\prime}\right)_{\left(x, x^{\prime}\right)}\right\urcorner=\bigoplus_{b} \circ\left\langle f \circ \pi_{1}, f \circ \pi_{2}\right\rangle=\ominus_{b} \circ(f \times f) \\
& \left\ulcorner x_{\left(x, x^{\prime}\right)}=x_{\left(x, x^{\prime}\right)}^{\prime}\right\urcorner=\bigoplus_{a} \circ\left\langle i d_{a} \circ \pi_{1}, i d_{a} \circ \pi_{2}\right\rangle=\ominus_{a} \circ i d_{a \times a}=\ominus_{a}
\end{aligned}
$$

(para $\pi_{1}, \pi_{2}$ as projeções do produto $a \times a$ )

Agora, seja $(p, \alpha, \beta)$ o kernel par de $f$. Mostremos que o quiadrado do diagrama abaixo é um pullback: sejam $\gamma_{1}: c \rightarrow b$ e $\gamma_{2}: c \rightarrow a \times a$ tais que $\Delta_{b} \circ \gamma_{1}=(f \times f) \circ \gamma_{2}$. Queremos 
achar uma única $\kappa: d \rightarrow p$ tornando o diagrama abaixo comutativo:

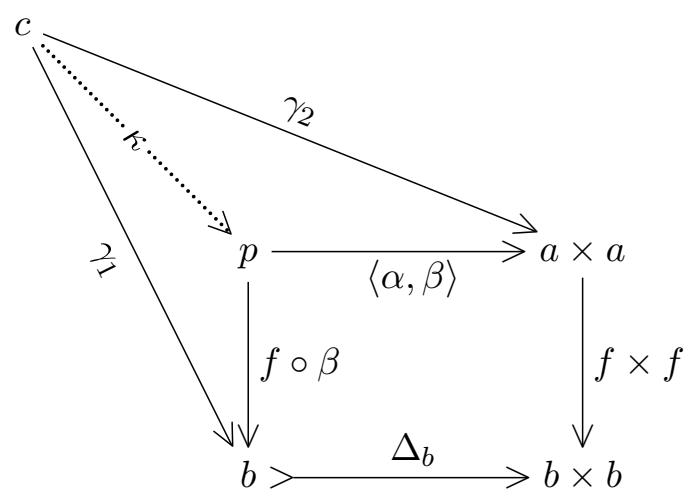

Por hipótese, temos $\left\langle\gamma_{1}, \gamma_{1}\right\rangle=\left\langle f \circ \pi_{1} \circ \gamma_{2}, f \circ \pi_{2} \circ \gamma_{2}\right\rangle$, ou seja, $f \circ \pi_{1} \circ \gamma_{2}=f \circ \pi_{2} \circ \gamma_{2}$. Assim, pelo pullback $(p, \alpha, \beta)$, existe uma única $\kappa: c \rightarrow p$ tal que $\beta \circ \kappa=\pi_{1} \circ \gamma_{2}$ e $\alpha \circ \kappa=\pi_{2} \circ \gamma_{2}$. Logo, $f \circ \beta \circ \kappa=f \circ \pi_{1} \circ \kappa=\gamma_{1}$, e $\langle\alpha, \beta\rangle \circ \kappa=\langle\alpha \circ \kappa, \beta \circ \kappa\rangle=\left\langle\pi_{1} \circ \gamma_{2}, \pi_{2} \circ \gamma_{2}\right\rangle=\gamma_{2}$, como queríamos.

Como no item anterior, o quadrado inferior do diagrama a seguir é um pullback:

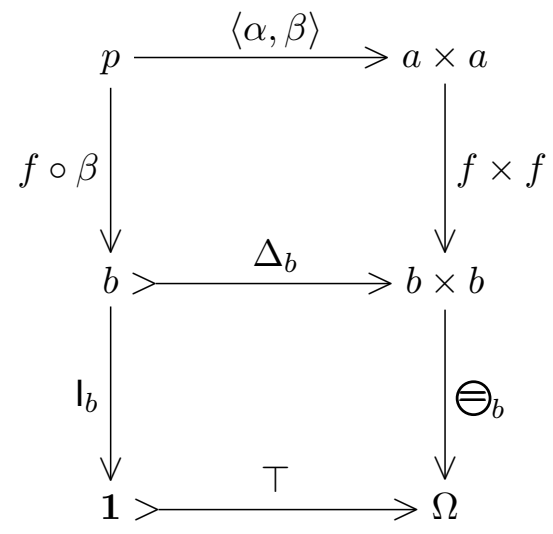

e o lema do pullback garante que o retângulo também o seja. Isto é, mostramos que $[\langle\alpha, \beta\rangle]=\llbracket f(x)_{\left(x, x^{\prime}\right)}=f\left(x^{\prime}\right)_{\left(x, x^{\prime}\right)} \rrbracket$.

É imediato da definição de $\bigoplus_{a}$ que $\left[\Delta_{a}\right]=\llbracket x_{\left(x, x^{\prime}\right)}=x_{\left(x, x^{\prime}\right)}^{\prime} \rrbracket$, e sempre vale que $\left[\Delta_{a}\right] \leq$ $[\langle\alpha, \beta\rangle]$ (basta usar as identidades no pullback que define o kernel par para obter uma flecha única $a \rightarrow p)$. Com isso, temos:

$$
\begin{aligned}
\mathcal{E} \models & \left(f(x)_{\left(x, x^{\prime}\right)}=f\left(x^{\prime}\right)_{\left(x, x^{\prime}\right)}\right) \Rightarrow\left(x_{\left(x, x^{\prime}\right)}=x_{\left(x, x^{\prime}\right)}^{\prime}\right) \\
& \Leftrightarrow \llbracket f(x)_{\left(x, x^{\prime}\right)}=f\left(x^{\prime}\right)_{\left(x, x^{\prime}\right)} \rrbracket \leq \llbracket x_{\left(x, x^{\prime}\right)}=x_{\left(x, x^{\prime}\right)}^{\prime} \rrbracket \\
& \Leftrightarrow[\langle\alpha, \beta\rangle] \leq\left[\Delta_{a}\right] \\
& \Leftrightarrow[\langle\alpha, \beta\rangle]=\left[\Delta_{a}\right] \\
& \Leftrightarrow f \text { é mono }
\end{aligned}
$$
(pelas contas do último parágrafo) (novamente pelo comentário acima) (pela proposição 1.5.8) 
Assim, o resultado do enunciado segue dos comentários do início da demonstração.

3. Como no item anterior, a igualdade é entre os termos com variáveis expandidas, e, chamando de $\pi_{1}$ e $\pi_{2}$ as projeções do produto $a \times b$, temos:

$$
\left\ulcorner f(x)_{(x, y)}=b_{(x, y)}\right\urcorner=\bigoplus_{b} \circ\left\langle f \circ \pi_{1}, i d_{b} \circ \pi_{2}\right\rangle=\ominus_{b} \circ\left(f \times i d_{b}\right)
$$

Agora, considere o diagrama:

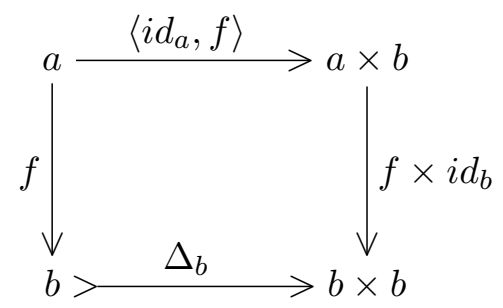

e mostremos que é um pullback. Sejam $\gamma_{1}: c \rightarrow b$ e $\gamma_{2}: c \rightarrow a \times b$ tais que $\Delta_{b} \circ \gamma_{1}=$ $\left(f \times i d_{b}\right) \circ \gamma_{2}$. Isso significa:

$$
\Delta_{b} \circ \gamma_{1}=\left\langle\gamma_{1}, \gamma_{1}\right\rangle=\left\langle f \circ \pi_{1} \circ \gamma_{2}, \pi_{2} \circ \gamma_{2}\right\rangle=\left\langle f \circ \pi_{1}, i d_{b} \circ \pi_{2}\right\rangle \circ \gamma_{2}=\left(f \times i d_{b}\right) \circ \gamma_{2}
$$

Assim, defina $\kappa:=\pi_{1} \circ \gamma_{2}: c \rightarrow a$, de forma que, pelas igualdades acima, $f \circ \kappa=\gamma_{1} \mathrm{e}$ $\left\langle i d_{a}, f\right\rangle \circ \kappa=\left\langle\pi_{1} \circ \gamma_{2}, \gamma_{1}\right\rangle=\left\langle\pi_{1} \circ \gamma_{2}, \pi_{2} \circ \gamma_{2}\right\rangle=\gamma_{2}$. É imediato que tal $\kappa$ é única, pois se existe $\rho: c \rightarrow a$ tal que $\left\langle i d_{a}, f\right\rangle \circ \rho=\gamma_{2}=\left\langle i d_{a}, f\right\rangle \circ \kappa$, então $\kappa=\rho$.

Mais uma vez usando a definição de $\bigoplus_{b}$ e o lema do pullback, obtemos que o retângulo abaixo é um pullback:

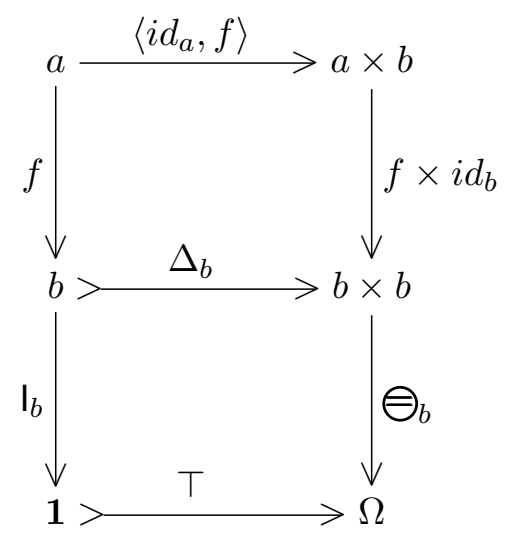

donde $\llbracket f(x)_{(x, y)}=b_{(x, y)} \rrbracket=\left\langle i d_{a}, f\right\rangle$.

Com isso, seja $\pi_{b}: a \times b \rightarrow b$ e projeção na segunda coordenada, e então:

$$
\exists_{\pi_{b}}\left(\llbracket f(x)_{(x, y)}=b_{(x, y)} \rrbracket\right)=\left[i m\left(\pi_{b} \circ\left\langle i d_{a}, f\right\rangle\right)\right]=[i m(f)]
$$


de forma que $\left\ulcorner\exists x\left(f(x)_{(x, y)}=b_{(x, y)}\right)\right\urcorner=\chi_{i m(f)}$.

Finalmente, pois, lembrando do item (1) que $\top_{b}=\chi_{i d_{b}}$, temos:

$$
\begin{aligned}
\mathcal{E} \models \exists x\left(f(x)_{(x, y)}=b_{(x, y)}\right) & \Leftrightarrow\left\ulcorner\exists x\left(f(x)_{(x, y)}=b_{(x, y)}\right)\right\urcorner=\top_{b} \\
& \Leftrightarrow \chi_{i m(f)}=\chi_{i d_{b}} \\
& \Leftrightarrow i m(f) \sim i d_{b} \\
& \Leftrightarrow f \text { é epi }
\end{aligned}
$$

sendo essa última equivalência decorrente da unicidade a menos de isomorfismo da fatoração epi-mono.

Lema 4.6.11. Sejam $\mathcal{E}$ um topos e $\varphi$ uma fórmula em $\mathcal{L}_{\mathcal{E}}$ com variável livre $x: X$. Então, $\ulcorner x \in\{x \mid \varphi\}\urcorner=\ulcorner\varphi\urcorner$.

Demonstração. Novamente o enunciado possui um abuso notação: $x \in\{x \mid \varphi\}$ na verdade significa $x_{()} \in\left\{x \mid \varphi_{()}\right\}_{(x)}$, para que os termos tenham mesmas variáveis e possamos definir a flecha que interpreta $\{x \mid \varphi\}$. Assim, sejam $\pi_{1}, \pi_{2}$ as projeções do produto $1 \times X$. Usando a definição de interpretação, temos:

$$
\begin{aligned}
\left\ulcorner x_{()} \in\left\{x \mid \varphi_{()}\right\}_{(x)}\right\urcorner & =e v_{\Omega} \circ\left\langle\left\ulcorner\left\{x \mid \varphi_{()}\right\}_{(x)}\right\urcorner,\left\ulcorner x_{()}\right\urcorner\right\rangle= \\
& \left.\left.=e v_{\Omega} \circ\left\langle\exp \left(\left\ulcorner\varphi_{()}\right\urcorner\right) \circ \pi_{1}, i d_{X} \circ \pi_{2}\right\rangle=e v_{\Omega} \circ\left(\exp \left(\left\ulcorner\varphi_{(}\right)\right\urcorner\right) \times i d_{X}\right)=\left\ulcorner\varphi_{(}\right)\right\urcorner
\end{aligned}
$$

sendo a última igualdade exatamente a definição da transposta exponencial.

No restante do capítulo, será comum denotarmos $\left\{x \mid \varphi_{()}\right\}$simplesmente por $\{x \mid \varphi\}$, para evitar sobrecarregar a notação.

Lema 4.6.12. Sejam $\mathcal{E}$ um topos e $\varphi$ uma fórmula em $\mathcal{L}_{\mathcal{E}}$ com variáveis livres $x_{1}: X_{1}, \ldots, x_{n}$ : $X_{n}$. Então, chamando $\bar{X}=X_{1} \times \ldots \times X_{n}$, os isomorfismos

$$
\operatorname{Sub}(\bar{X}) \cong \mathcal{E}(\bar{X}, \Omega) \cong \mathcal{E}\left(\mathbf{1}, \Omega^{\bar{X}}\right)
$$

fornecem uma correspondência entre:

$$
\llbracket \varphi \rrbracket \leftrightarrow\ulcorner\varphi\urcorner \quad \leadsto \quad\left\ulcorner\left\{x_{1}, \ldots, x_{n} \mid \varphi\right\}\right\urcorner
$$


Demonstração. A correspondência entre $\llbracket \varphi \rrbracket$ e $\ulcorner\varphi\urcorner$ é simplesmente a definição. A outra correspondência também é imediata, pois $\left\ulcorner\left\{x_{1}, \ldots, x_{n} \mid \varphi\right\}\right\urcorner:=\exp (\ulcorner\varphi\urcorner): \mathbf{1} \rightarrow \Omega^{\bar{X}}$, e $h \mapsto \exp (h)$ é exatamente o isomorfismo $\mathcal{E}(\bar{X}, \Omega) \cong \mathcal{E}\left(\mathbf{1}, \Omega^{\bar{X}}\right)$.

Proposição 4.6.13. Sejam $\mathcal{E}$ um topos e $\varphi, \psi$ fórmulas em $\mathcal{L}_{\mathcal{E}}$ com variável livre $x: X$. Então, pelo isomorfismo $\mathcal{E}(X, \Omega) \cong \mathcal{E}\left(\mathbf{1}, \Omega^{X}\right)$, temos as correspondências:

$$
\begin{aligned}
& \text { 1. }\ulcorner\varphi \wedge \psi\urcorner \leadsto\ulcorner\{x \mid(x \in\{x \mid \varphi\}) \wedge(x \in\{x \mid \psi\})\}\urcorner ; \\
& \text { 2. }\ulcorner\varphi \vee \psi\urcorner \rightsquigarrow\ulcorner\{x \mid(x \in\{x \mid \varphi\}) \vee(x \in\{x \mid \psi\})\}\ulcorner\text {; } \\
& \text { 3. }\ulcorner\varphi \Rightarrow \psi\urcorner \leadsto\ulcorner\{x \mid(x \in\{a \mid \varphi\}) \Rightarrow(x \in\{x \mid \psi\})\}\urcorner \text {; } \\
& \text { 4. }\ulcorner\neg \varphi\urcorner \leadsto\ulcorner\{x \mid \neg(x \in\{x \mid \varphi\})\}\urcorner \text {. }
\end{aligned}
$$

Demonstração. Todos são imediatos dos dois lemas anteriores. Por exemplo, no primeiro item, pela definição de interpretação e pelo lema 4.6.11 (e com o abuso de notação nele citado), temos:

$$
\ulcorner\varphi \wedge \psi\urcorner=\ulcorner\varphi\urcorner \wedge\ulcorner\psi\urcorner=\ulcorner x \in\{x \mid \varphi\}\urcorner \wedge\ulcorner x \in\{x \mid \psi\}\urcorner=\ulcorner x \in\{x \mid \varphi\} \wedge x \in\{x \mid \psi\}\urcorner
$$

Logo, pelo lema 4.6.12, $\ulcorner\varphi \wedge \psi\urcorner$ corresponde a $\ulcorner\{x \mid(x \in\{x \mid \varphi\}) \wedge(x \in\{x \mid \psi\})\}\urcorner$.

Por causa das correspondências do lema anterior, alguns autores costumam denotar todos esses elementos correspondentes por um mesmo símbolo e substituir a correspondência por igualdade, o que deixa essa proposição com um aspecto mais familiar (embora ao mesmo tempo contribua para obscurecer seu significado preciso). Por exemplo, denotando dois subobjetos por $S_{1}$ e $S_{2}$, o primeiro item pode ser reescrito como:

$$
S_{1} \wedge S_{2}=\left\{x \mid\left(x \in S_{1}\right) \wedge\left(x \in S_{2}\right)\right\}
$$

Para finalizar a seção, façamos alguns comentários sobre abordagens alternativas e outros desenvolvimentos da lógica de topos.

Uma dessas abordagens, feita em [Bel88], usa teorias de conjuntos locais. No cerne dessa abordagem está o fato que topoi servem de modelo para teorias de conjuntos locais, e que todo topos é equivalente a uma categoria linguística (que é uma categoria determinada por uma teoria de conjuntos local, tal como a teoria dos conjuntos clássica determina Set). 
Em [MR77] é apresentada uma abordagem um pouco mais próxima do que foi feito no presente trabalho, mas com um foco em relacionar teoria dos modelos de primeira ordem com teoria de categorias, especialmente com topoi de Grothendieck. Em particular, os autores mostram como algumas propriedades fundamentais de noções da teoria de topos podem ser obtidas puramente através da lógica.

Essa abordagem está intimamente relacionada com a teoria dos topoi classificantes. Primeiro, considere uma noção de "estrutura" matemática (por exemplo, grupos, anéis, etc.) tal que todo topos $\mathcal{E}$ possui uma categoria dessas estruturas em $\mathcal{E}$ (como a categoria dos "objetos de grupo" ou "objetos de anel" num topos). Um topos $\mathcal{T}$ é um topos classificante para essa estrutura se existe uma equivalência natural entre os morfismos geométricos $\mathcal{E} \rightarrow \mathcal{T}$ e os objetos dessa estrutura em $\mathcal{E}$. Um exemplo de topos classificante é o Topos de Zariski, que classifica os objetos de anel local num topos.

Um resultado importante sobre topoi classificantes é que toda estrutura que pode ser axiomatizada por uma teoria geométrica ${ }^{4}$ possui um topos classificante. Recomenda-se consultar o capítulo D3 de [Joh02b], os capítulos VIII e X de [LM94] ou o capítulo 9 de [MR77] para uma apresentação mais completa sobre topoi classificantes.

\subsection{Semântica de Kripke-Joyal}

Com inspiração no "caráter local" das propriedades dos feixes, a ideia da semântica de KripkeJoyal é que a satisfação de uma fórmula seja determinada pela sua satisfação local. Escrevamos isso de forma precisa, usando estágios e elementos, para então mostrar como isso se relaciona com a validade de fórmulas definida acima.

Definição 4.7.1. Seja $\mathcal{E}$ um topos com subclasse geradora $\mathcal{G} \subseteq \mathcal{E}_{0}$. Chamamos os elementos de $\mathcal{G}$ de estágios. Uma $\mathcal{G}$-cobertura de um estágio $u \in \mathcal{G}$ é uma família epimórfica $\left\{h_{i}: u_{i} \rightarrow u \mid\right.$ $\left.i \in I, u_{i} \in \mathcal{G}\right\}$.

Definição 4.7.2. Seja $\mathcal{E}$ um topos com subclasse geradora $\mathcal{G} \subseteq \mathcal{E}_{0}$. Um elemento de um objeto $A \in \mathcal{E}_{0}$ num estágio $u \in \mathcal{G}$ é uma flecha $a: u \rightarrow A$ em $\mathcal{E}_{1}$. Nesse caso, denotamos $a \in_{u} A$.

Definição 4.7.3. Sejam $\mathcal{E}$ um topos com subclasse geradora $\mathcal{G} \subseteq \mathcal{E}_{0}$ e $t: A$ um termo em $\mathcal{L}_{\mathcal{E}}$ com variáveis livres $x_{1}: X_{1}, \ldots, x_{n}: X_{n}$. Para cada estágio $u \in \mathcal{G}$ e elementos $a_{1} \in_{u} X_{1}, \ldots, a_{n} \in_{u} X_{n}$,

\footnotetext{
${ }^{4}$ Uma fórmula de primeira ordem é dita geométrica se é construída usando apenas conjunção, disjunção e o quantificador existencial. Uma teoria geométrica é uma teoria cujos axiomas são da forma $\forall x(\varphi(x) \Rightarrow \psi(x))$, para $\varphi, \psi$ fórmulas geométricas.
} 
definimos o elemento $t\left(a_{1}, \ldots, a_{n}\right) \in_{u} A$ como $t\left(a_{1}, \ldots, a_{n}\right):=\ulcorner t\urcorner \circ\left\langle a_{1}, \ldots, a_{n}\right\rangle$.

Definição 4.7.4. Sejam $\mathcal{E}$ um topos com subclasse geradora $\mathcal{G} \subseteq \mathcal{E}_{0}$ e $\varphi: \Omega$ uma fórmula em $\mathcal{L}_{\mathcal{E}}$ com variáveis livres $x_{1}: X_{1}, \ldots, x_{n}: X_{n}$. Para cada estágio $u \in \mathcal{G}$ e elementos $a_{1} \in_{u} X_{1}, \ldots, a_{n} \in_{u}$ $X_{n}$, dizemos que $\left(a_{1}, \ldots, a_{n}\right)$ satisfaz (ou força) $\varphi$ se $\varphi\left(a_{1}, \ldots, a_{n}\right)=\top_{u}$, e nesse caso denotaremos $u \Vdash \varphi\left(a_{1}, \ldots, a_{n}\right)$.

A ideia da relação $\Vdash$, portanto, é definir a "satisfação de fórmulas através de elementos de estágios". O teorema a seguir garante que essa satisfação corresponde à validade de fórmulas na linguagem de Mitchell-Bénabou.

Teorema 4.7.5. Sejam $\mathcal{E}$ um topos com subclasse geradora $\mathcal{G} \subseteq \mathcal{E}_{0}$ e $\varphi: \Omega$ uma fórmula com variáveis livres $x_{1}: X_{1}, \ldots, x_{n}: X_{n}$. Então, são equivalentes:

1. $\mathcal{E}=\varphi ;$

2. para todo $u \in \mathcal{G}$, para todos elementos $a_{1} \in_{u} X_{1}, \ldots, a_{n} \in_{u} X_{n}, u \Vdash \varphi\left(a_{1}, \ldots, a_{n}\right)$.

Demonstração. $(1 \Rightarrow 2) \mathcal{E} \models \varphi$ significa $\ulcorner\varphi\urcorner=\top_{X_{1} \times \ldots \times X_{n}}$. Para todo $u \in \mathcal{G}$, para todos elemen$\operatorname{tos} a_{1} \in_{u} X_{1}, \ldots, a_{n} \in_{u} X_{n}$, definimos $\varphi\left(a_{1}, \ldots, a_{n}\right)=\ulcorner\varphi\urcorner \circ\left\langle a_{1}, \ldots, a_{n}\right\rangle$, portanto $\varphi\left(a_{1}, \ldots, a_{n}\right)=\top_{u}$ e, por definição, $u \Vdash \varphi\left(a_{1}, \ldots, a_{n}\right)$.

$(2 \Rightarrow 1)$ Considere os morfismos $\ulcorner\varphi\urcorner, \top_{X_{1} \times \ldots \times X_{n}}: X_{1} \times \ldots \times X_{n} \rightarrow \Omega$. Para cada estágio $u \in \mathcal{G}$ e toda flecha $a: u \rightarrow X_{1} \times \ldots \times X_{n}$, defina $a_{i}=\pi_{i} \circ a$, para cada $i \in\{1, \ldots, n\}\left(\operatorname{com} \pi_{i}\right.$ a $i$-ésima projeção do produto $\left.X_{1} \times \ldots \times X_{n}\right)$. Assim, a hipótese nos fornece:

$$
\ulcorner\varphi\urcorner \circ a=\ulcorner\varphi\urcorner \circ\left\langle\pi_{1}, \ldots, \pi_{n}\right\rangle \circ a=\ulcorner\varphi\urcorner \circ\left\langle a_{1}, \ldots, a_{n}\right\rangle=\top_{u}=\top_{X_{1} \times \ldots \times X_{n}} \circ a
$$

Como $\mathcal{G}$ é subclasse geradora, isso implica em $\ulcorner\varphi\urcorner=\top_{X_{1} \times \ldots \times X_{n}}, \log \mathcal{E} \models \varphi$.

Finalmente, desenvolvendo as propriedades de $u \Vdash \varphi\left(a_{1}, \ldots, a_{n}\right)$ usando a construção indutiva da fórmula $\varphi$, obtemos uma coleção de regras semânticas, conhecidas, em conjunto, como a semântica de Kripke-Joyal. Abaixo, apresentamos essas regras:

Proposição 4.7.6. Sejam $\mathcal{E}$ um topos com subclasse geradora $\mathcal{G} \subseteq \mathcal{E}_{0}, \varphi: \Omega$ e $\psi: \Omega$ fórmulas com variáveis livres $x_{1}: X_{1}, \ldots, x_{n}: X_{n}$, e $t: A, s: A$ e $\Sigma: \Omega^{A}$ termos com variáveis livres $x_{1}: X_{1}, \ldots, x_{n}: X_{n}$. Então, para todos $u, v \in \mathcal{G}$ e para todos elementos $a_{1} \in_{u} X_{1}, \ldots, a_{n} \in_{u} X_{n}$, vale: 
1. (monotonicidade) para toda $f: v \rightarrow u$ em $\mathcal{E}_{1}$, se $u \Vdash \varphi\left(a_{1}, \ldots, a_{n}\right)$, então $v \Vdash \varphi\left(a_{1} \circ\right.$ $\left.f, \ldots, a_{n} \circ f\right)$;

2. (caráter local) para toda $f: v \rightarrow u$ em $\mathcal{E}_{1}$, se $v \Vdash \varphi\left(a_{1} \circ f, \ldots, a_{n} \circ f\right)$ e $f$ é epi, então $u \Vdash \varphi\left(a_{1}, \ldots, a_{n}\right) ;$

3. $s e\ulcorner\varphi\urcorner=\top$, então $u \Vdash \varphi\left(a_{1}, \ldots, a_{n}\right)$;

4. se $\ulcorner\varphi\urcorner=\perp$, então $u \Vdash \varphi\left(a_{1}, \ldots, a_{n}\right)$ se, e somente se, $u \cong \mathbf{0}$;

5. $u \Vdash(t=s)\left(a_{1}, \ldots, a_{n}\right)$ se, e somente se, $t\left(a_{1}, \ldots, a_{n}\right)=s\left(a_{1}, \ldots, a_{n}\right)$;

6. para todos elementos $a_{n+1} \in_{u} X_{n+1}, \ldots, a_{n+m} \in_{u} X_{n+m}, u \Vdash \varphi_{\left(x_{1}, \ldots, x_{n+m}\right)}\left(a_{1}, \ldots, a_{n+m}\right) s e$, e somente se, $u \Vdash \varphi\left(a_{1}, \ldots, a_{n}\right)$;

7. para cada $i \in\{1, \ldots, n\}$, seja $t_{1}: X_{i}$ um termo com variáveis livres $y_{1}: Y_{1}, \ldots, y_{m}: Y_{m}$. Então, para todos elementos $b_{1} \in_{u} Y_{1}, \ldots, b_{m} \in_{u} Y_{m}$, temos $u \Vdash\left(\varphi\left(t_{1}, \ldots, t_{n}\right)\right)\left(b_{1}, \ldots, b_{m}\right)$ se, e somente se, $u \Vdash \varphi\left(t_{1}\left(b_{1}, \ldots, b_{m}\right), \ldots, t_{n}\left(b_{1}, \ldots, b_{m}\right)\right)$;

8. chamando de $\sigma: M \longmapsto u \times A$ uma flecha correspondente a $\Sigma\left(a_{1}, \ldots, a_{n}\right): u \rightarrow \Omega^{A}$ (pelas bijeções $\left.S u b(u \times A) \cong \mathcal{E}(u \times A, \Omega) \cong \mathcal{E}\left(u, \Omega^{A}\right)\right)$, temos $u \Vdash(t \in \Sigma)\left(a_{1}, \ldots, a_{n}\right)$ se, e somente se, existe $f: u \rightarrow M$ em $\mathcal{E}_{1}$ tal que $\sigma \circ f=\left\langle i d_{U}, t\left(a_{1}, \ldots, a_{n}\right)\right\rangle$ (ou, equivalentemente, $\left.\left(\Sigma\left(a_{1}, \ldots, a_{n}\right) \times i d_{A}\right) \circ \sigma \circ f=\left\langle\Sigma\left(a_{1}, \ldots, a_{n}\right), t\left(a_{1}, \ldots, a_{n}\right)\right\rangle\right) ;$

9. $u \Vdash(\varphi \wedge \psi)\left(a_{1}, \ldots, a_{n}\right)$ se, e somente se, $u \Vdash \varphi\left(a_{1}, \ldots, a_{n}\right)$ e $u \Vdash \psi\left(a_{1}, \ldots, a_{n}\right)$;

10. $u \Vdash(\varphi \Rightarrow \psi)\left(a_{1}, \ldots, a_{n}\right)$ se, e somente se, para todo $w \in \mathcal{G}$ e toda $f: w \rightarrow u$ em $\mathcal{E}_{1}$ vale: se $w \Vdash \varphi\left(a_{1} \circ f, \ldots, a_{n} \circ f\right)$ então $w \Vdash \psi\left(a_{1} \circ f, \ldots, a_{n} \circ f\right)$;

11. $u \Vdash \neg \varphi\left(a_{1}, \ldots, a_{n}\right)$ se, e somente se, $u \Vdash(\varphi \Rightarrow \perp)\left(a_{1}, \ldots, a_{n}\right)$ (ou, equivalentemente, usando itens anteriores, para toda $w \in \mathcal{G}$ e toda $f: w \rightarrow u$ em $\mathcal{E}_{1}$ vale: $w \Vdash \varphi\left(a_{1} \circ f, \ldots, a_{n} \circ f\right)$ se, e somente se, $w \cong \mathbf{0}$ );

12. $u \Vdash(\varphi \vee \psi)\left(a_{1}, \ldots, a_{n}\right)$ se, e somente se, existe uma $\mathcal{G}$-cobertura $\left\{h_{i}: u_{i} \rightarrow u \mid i \in I, u_{i} \in \mathcal{G}\right\}$ de u tal que $u_{i} \Vdash \varphi\left(a_{1} \circ h_{i}, \ldots, a_{n} \circ h_{i}\right)$ ou $u_{i} \Vdash \psi\left(a_{1} \circ h_{i}, \ldots, a_{n} \circ h_{i}\right)$ para todo $i \in I$;

13. seja $\phi: \Omega$ uma fórmula com variáveis livres $x: X, x_{1}: X_{1}, \ldots, x_{n}: X_{n}$ (com $x \neq x_{i}$, para todo $i \in\{1, \ldots, n\})$. Então:

(a) $u \Vdash(\exists x \phi)\left(a_{1}, \ldots, a_{n}\right)$ se, e somente se, existe uma $\mathcal{G}$-cobertura $\left\{h_{i}: u_{i} \rightarrow u \mid i \in\right.$ $\left.I, u_{i} \in \mathcal{G}\right\}$ de u tal que para todo $i \in I$ existe $b_{i} \in_{u} X$ tal que $u_{i} \Vdash \phi\left(b_{i}, a_{1} \circ h_{i}, \ldots, a_{n} \circ\right.$ $\left.h_{i}\right)$; 
(b) $u \Vdash(\forall x \phi)\left(a_{1}, \ldots, a_{n}\right)$ se, e somente se, para todo $w \in \mathcal{G}$, toda $f: w \rightarrow u$ em $\mathcal{E}_{1}$ e todo $b \in_{u} X$ temos $w \Vdash \phi\left(b, a_{1} \circ f, \ldots, a_{n} \circ f\right)$.

Para a verificação desses resultados, recomendamos ver a seção 6.6 de [Bor08c] ou a seção VI.6 de [LM94]. Façamos aqui apenas um deles como exemplo.

Demonstração. 12. Primeiramente, chamando $\varphi(\vec{a}):=\varphi\left(a_{1}, \ldots, a_{n}\right)$ e $\psi(\vec{a}):=\psi\left(a_{1}, \ldots, a_{n}\right)$, façamos os pullbacks de $\left\langle\top, i d_{\Omega}\right\rangle$ e $\left\langle i d_{\Omega}, T\right\rangle$ ao longo de $\langle\varphi(\vec{a}), \psi(\vec{a})\rangle$ como nos quadrados do diagrama abaixo:

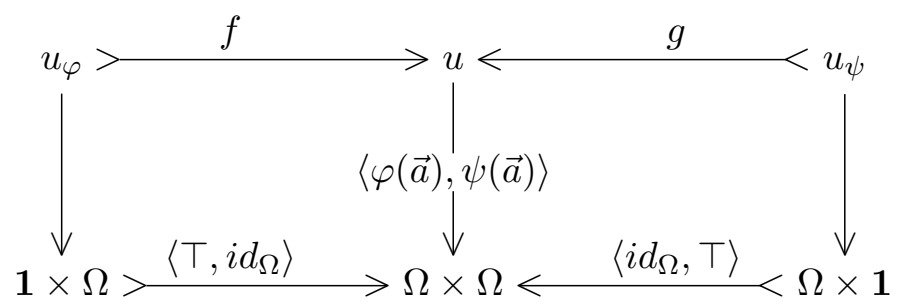

Usando que coprodutos são estáveis sob pullbacks (ver 3.4.7), obtemos o pullback:

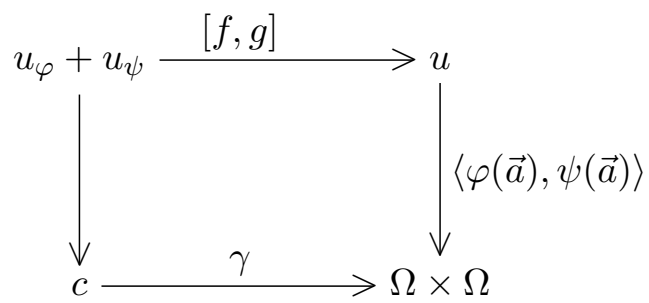

onde chamamos $c:=(\mathbf{1} \times \Omega)+(\Omega \times \mathbf{1})$ e $\gamma:=\left[\left\langle\top, i d_{\Omega}\right\rangle,\left\langle i d_{\Omega}, \top\right\rangle\right]$. Com isso, usando o lema 4.2.7, existe uma única flecha $\kappa:[f, g]\left(u_{\varphi}+u_{\psi}\right) \rightarrow \gamma(c)$ que torna o quadrado superior do diagrama abaixo um pullback:

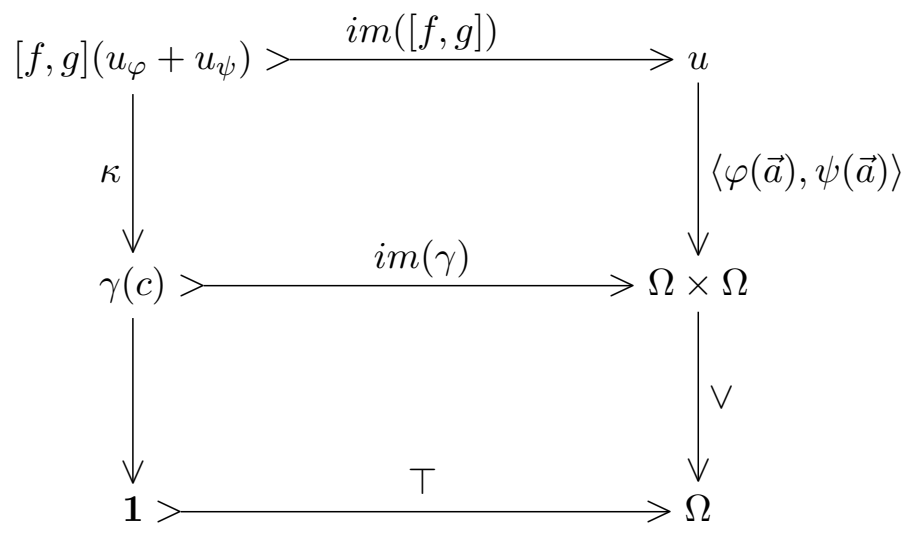

O quadrado inferior é um pullback pela própria definição da disjunção, logo, pelo lema do pullback, o retângulo também é um pullback. Feito esse preâmbulo, mostremos as implicações do enunciado. 
$(\Rightarrow)$ Abrindo as definições, temos as igualdades:

$$
(\varphi \vee \psi)(\vec{a})=\ulcorner\varphi \vee \psi\urcorner \circ\left\langle a_{1}, \ldots, a_{n}\right\rangle=\vee \circ\langle\ulcorner\varphi\urcorner,\ulcorner\psi\urcorner\rangle \circ\left\langle a_{1}, \ldots, a_{n}\right\rangle=\vee \circ\langle\varphi(\vec{a}), \psi(\vec{a})\rangle
$$

logo, a hipótese $u \Vdash(\varphi \vee \psi)\left(a_{1}, \ldots, a_{n}\right)$ implica em $\vee \circ\langle\varphi(\vec{a}), \psi(\vec{a})\rangle=(\varphi \vee \psi)(\vec{a})=\top_{u}$.

Como o retângulo do diagrama (2) é um pullback e $\top_{u}=\chi_{i d_{u}}$, temos que $i m([f, g]) \sim i d_{u}$. Logo, $[f, g]=i m([f, g]) \circ[f, g]^{\triangleright}$ é um epi. Com isso, mostremos que $\{f, g\}$ é uma família epimórfica. Sejam $h, k: u \rightarrow b$ tais que $h \circ f=k \circ f$ e $h \circ g=k \circ g$. Considere o diagrama de coproduto, onde denotamos $h \circ f=\tilde{f}$ e $h \circ g=\tilde{g}$

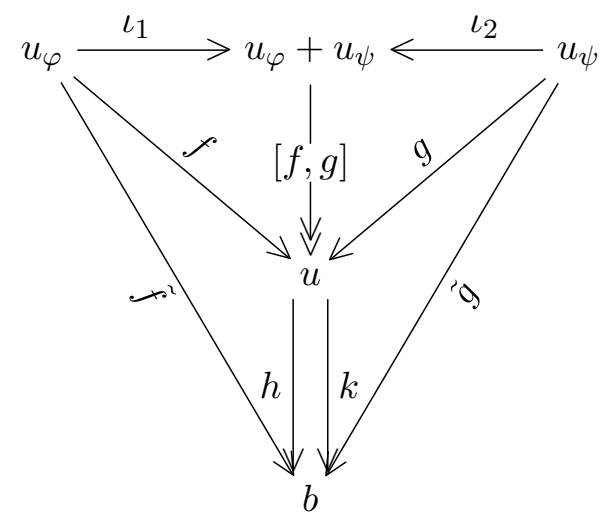

Pela unicidade da flecha do coproduto, obtemos $h \circ[f, g]=[\tilde{f}, \tilde{g}]=k \circ[f, g]$, e usando que $[f, g]$ é epi, conlcluímos que $h=k$; ou seja, $\{f, g\}$ é epimórfica.

Além disso, como $\mathcal{G}$ é subclasse geradora, as famílias $R=\left\{r: v \rightarrow u_{\varphi} \mid v \in \mathcal{G}, r \in \mathcal{E}_{1}\right\} \mathrm{e}$ $S=\left\{s: w \rightarrow u_{\psi} \mid w \in \mathcal{G}, s \in \mathcal{E}_{1}\right\}$ também são epimórficas, logo a família

$$
C=\{f \circ r \mid r \in R\} \cup\{g \circ s \mid s \in S\}
$$

é uma $\mathcal{G}$-cobertura de $u$. Assim, para cada $t \in C$, temos dois casos: $t=f \circ r: v \rightarrow u$ (para algum $r \in R$ ) ou $t=g \circ s: w \rightarrow u$ (para algum $s \in S$ ). Usando a comutatividade do diagrama (1), no primeiro caso temos:

$$
\varphi\left(a_{1} \circ t, \ldots, a_{n} \circ t\right)=\ulcorner\varphi\urcorner \circ\left\langle a_{1}, \ldots, a_{n}\right\rangle \circ f \circ r=\top \circ \mathrm{I}_{u_{\varphi}} \circ r=\top_{v}
$$

donde $v \Vdash \varphi\left(a_{1} \circ t, \ldots, a_{n} \circ t\right)$. Similarmente, no segundo caso temos:

$$
\psi\left(a_{1} \circ t, \ldots, a_{n} \circ t\right)=\ulcorner\psi\urcorner \circ\left\langle a_{1}, \ldots, a_{n}\right\rangle \circ g \circ s=\top \circ \mathrm{I}_{u_{\psi}} \circ s=\top_{w}
$$

ou seja, $w \Vdash \psi\left(a_{1} \circ t, \ldots, a_{n} \circ t\right)$, como queríamos. 
$(\Leftarrow)$ Para cada $i \in I$, considere $p$ o (objeto do) pullback de $f$ ao longo de $h_{i}$, e $q$ o (objeto do) pullback de $g$ ao longo de $h_{i}$, como nos quadrados superiores dos diagramas abaixo:
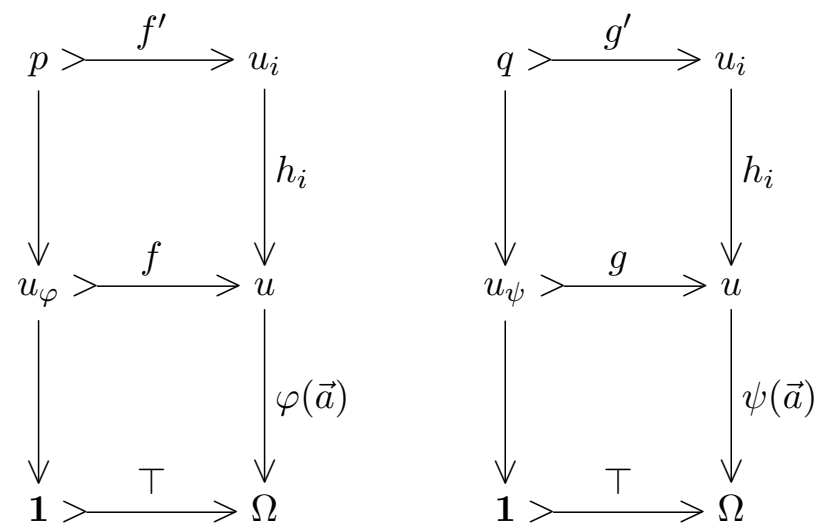

Por definição, os quadrados inferiores também são pullbacks (ver diagrama (1)), logo os retângulos também serão pullbacks. Assim, se $u_{i} \Vdash \varphi\left(a_{1} \circ h_{i}, \ldots, a_{n} \circ h_{i}\right)$, então:

$$
\varphi(\vec{a}) \circ h_{i}=\ulcorner\varphi\urcorner \circ\left\langle a_{1}, \ldots, a_{n}\right\rangle \circ h_{i}=\varphi\left(a_{1} \circ h_{i}, \ldots, a_{n} \circ h_{i}\right)=\top_{u_{i}}
$$

e, usando que o retângulo da esquerda é um pullback e que $\top_{u_{i}}=\chi_{i d_{u_{i}}}$, obtemos $f^{\prime} \sim i d_{u_{i}}$. Note que isso implica em $\left[f^{\prime}, g^{\prime}\right]: p+q \rightarrow u_{i}$ ser epi. De fato, chamando de $\iota_{1}^{\prime}: p \rightarrow$ $p+q$ a primeira injeção canônica, se $h, k \in \mathcal{E}_{1}$ satisfazem $h \circ\left[f^{\prime}, g^{\prime}\right]=k \circ\left[f^{\prime}, g^{\prime}\right]$, então $h \circ\left[f^{\prime}, g^{\prime}\right] \circ \iota_{1}^{\prime}=k \circ\left[f^{\prime}, g^{\prime}\right] \circ \iota_{1}^{\prime}$, que por definição significa $h \circ f^{\prime}=k \circ f^{\prime}$. Logo, como $f^{\prime}$ é iso, concluímos $h=k$.

Agora, fazendo uma construção semelhante à implicação $(\Rightarrow)$, obtemos que o quadrado superior do diagrama abaixo é um pullback:

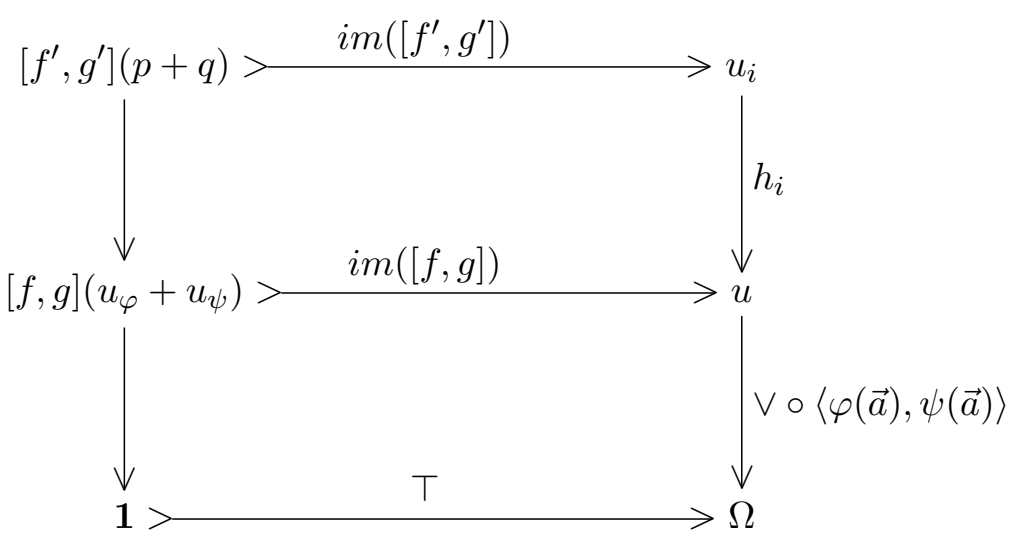

O quadrado inferior é simplesmente o diagrama (2), que já mostramos ser um pullback; e novamente o lema do pullback garante que o retângulo seja um pullback.

Finalmente, como $\left[f^{\prime}, g^{\prime}\right]$ é epi, $i m\left(\left[f^{\prime}, g^{\prime}\right]\right)$ é iso, isto é, $i m\left(\left[f^{\prime}, g^{\prime}\right]\right) \sim i d_{u_{i}}$. Portanto:

$$
(\varphi \vee \psi)(\vec{a}) \circ h_{i}=\vee \circ\langle\varphi(\vec{a}), \psi(\vec{a})\rangle h_{i}=\chi_{i d_{u_{i}}}=\top_{u_{i}}=\top_{u} \circ h_{i}
$$


Assim, como isso vale para todo $i \in I$ e os $h_{i}$ formam uma família epimórfica, obtemos $(\varphi \vee \psi)(\vec{a})=\top_{u}$; isto é, $u \Vdash(\varphi \vee \psi)(\vec{a})$.

O caso para $u_{i} \Vdash \psi\left(a_{1} \circ h_{i}, \ldots, a_{n} \circ h_{i}\right)$ é obviamente análogo.

Exemplo 4.7.7. Num topos $\mathcal{E}=\operatorname{Sh}(\mathcal{C}, J)$, com $(\mathcal{C}, J)$ um sítio pequeno, pode-se mostrar, usando que todo prefeixe é colimite de funtores representáveis (teorema 1.5.19), que $\left\{a_{0}(\mathcal{C}(-, c)) \mid\right.$ $\left.c \in \mathcal{C}_{0}\right\}$ forma um conjunto gerador (para $a=(-)^{++}$, definido no exemplo 2.3.19). Agora, para um feixe $F$ sobre $(\mathcal{C}, J)$, os elementos de $F$ num estágio $a_{0}(\mathcal{C}(-, c))$ são transformações naturais $\eta: a_{0}(\mathcal{C}(-, c)) \Rightarrow F$. Assim, usando o Lema de Yoneda e que $a \dashv i$, temos:

$$
\mathcal{E}\left(a_{0}(\mathcal{C}(-, c)), F\right) \cong \mathcal{E}\left(\mathcal{C}(-, c), i_{0}(F)\right) \cong \mathcal{E}(\mathcal{C}(-, c), F) \cong F_{0}(c)
$$

ou seja, elementos de $F$ num estágio $a_{0}(\mathcal{C}(-, c))$ correspondem a elementos de $F_{0}(c)$.

No caso de $\mathbf{S h}(L)$, a categoria de feixes sobre um locale, as coberturas de locales formam uma topologia canônica, logo os próprios funtores $L(-, c)$ formam uma subclasse geradora. Por isso, dada uma fórmula $\varphi$ com variáveis livres $x_{1}: F_{1}, \ldots, x_{n}: F_{n}$ e elementos $b_{1} \in\left(F_{1}\right)_{0}(c), \ldots, b_{n} \in$ $\left(F_{n}\right)_{0}(c)$, é comum denotarmos $L(-, c) \Vdash \varphi\left(b_{1}, \ldots, b_{n}\right)$ apenas por $c \Vdash \varphi\left(b_{1}, \ldots, b_{n}\right)$. Além disso, $c \Vdash \varphi\left(b_{1}, \ldots, b_{n}\right)$ significa que $\ulcorner\varphi\urcorner_{c}\left(b_{1}, \ldots, b_{n}\right)=c$, ou seja, que $\left(b_{1}, \ldots, b_{n}\right) \in \llbracket \varphi \rrbracket_{0}(c)$, para $\llbracket \rrbracket$ o subfuntor de $F_{1} \times \ldots \times F_{n}$ correspondente a $\ulcorner\varphi\urcorner$.

\subsection{Objeto números naturais num topos}

Tendo disponível agora a lógica de topos, pode-se mostrar que, num topos, os objetos números naturais correspondem à axiomática de Peano.

Definição 4.8.1. Seja $\mathcal{E}$ um topos. Um modelo da aritmética de Peano em $\mathcal{E}$ é uma tripla $\left(\mathbb{N}_{\mathcal{C}}, 0_{\mathcal{C}}, s\right)$, onde $\mathbb{N}_{\mathcal{C}} \in \mathcal{C}_{0}$ e $0_{\mathcal{C}}: \mathbf{1} \rightarrow \mathbb{N}_{\mathcal{C}}$ e $\mathbb{N}_{\mathcal{E}}$ e $s: \mathbb{N}_{\mathcal{C}} \rightarrow \mathbb{N}_{\mathcal{C}}$ são flechas em $\mathcal{E}_{1}$, tal que:

(P1) $\mathcal{E} \models\left(n=\overline{0_{\mathcal{C}}}\right) \vee \exists m(m=s(n)) ;$

(P2) $\mathcal{E} \models \neg\left(s(n)=\overline{0_{\mathcal{C}}}\right)$;

(P3) $\mathcal{E} \models(s(n)=s(m)) \Rightarrow(n=m)$;

(P4) se $\mathcal{E} \models\left(\overline{0_{\mathcal{C}}} \in\{n \mid \varphi\}\right) \wedge \forall n((n \in\{n \mid \varphi\}) \Rightarrow(s(n) \in\{n \mid \varphi\}))$, então $\llbracket \varphi \rrbracket=\left[i d_{\mathbb{N}_{\mathcal{E}}}\right]$; 
para $n: \mathbb{N}_{\mathcal{E}}$ e $m: \mathbb{N}_{\mathcal{E}}$ variáveis, e $\varphi$ uma fórmula com variável livre $n$.

Como nos comentários após a proposição 4.6.13, alguns autores adotam um abuso de notação e escrevem essa última condição de forma mais familiar (para $P$ um subobjeto):

se $\mathcal{E}=\left(\overline{0_{\mathcal{C}}} \in P\right) \wedge \forall n((n \in P) \Rightarrow(s(n) \in P))$, então $P=\mathbb{N}_{\mathcal{E}}$

Proposição 4.8.2. Funtores lógicos preservam modelos da aritmética de Peano.

Demonstração. Como funtores lógicos preservam a validade de fórmulas (ver proposição 4.6.6), as propriedades $(\mathbf{P} 1)$ a $(\mathbf{P} 4)$ também são preservadas. Só falta mostrar que a flecha $0_{\mathcal{E}}: \mathbf{1} \rightarrow \mathbb{N}_{\mathcal{E}}$ é levada a uma flecha que também tem um objeto terminal como domínio, mas esse é o caso pois, por definição, funtores lógicos preservam limites finitos.

Para não nos alongarmos demasiadamente no assunto, não incluiremos aqui a demonstração da correspondência entre objetos números naturais e modelos de aritmética de Peano (como referência, ver proposições 8.1.10 e 8.1.12 de [Bor08c]). Porém, como ilustração do uso da lógica no contexto dos números naturais, façamos o princípio da indução.

Proposição 4.8.3. Princípio da indução. Sejam $\mathcal{E}$ um topos com modelo da aritmética de Peano $\left(\mathbb{N}_{\mathcal{E}}, 0_{\mathcal{E}}, s\right)$ e $\varphi: \Omega$ uma fórmula com variável livre $n: \mathbb{N}_{\mathcal{E}}$. Então, são equivalentes:

1. $\mathcal{E} \models \varphi$;

2. $\mathcal{E} \models \varphi\left(\overline{0_{\mathcal{E}}}\right)$ e $\mathcal{E} \models \varphi(n) \Rightarrow \varphi(s(n))$.

Demonstração. $(\Rightarrow)$ Por generalização universal (ver teorema 4.6.8), se $\mathcal{E} \models \varphi$, então $\mathcal{E} \models \forall m \varphi$, para $m: \mathbb{N}_{\mathcal{E}}$ uma variável. Agora pelo item (p) do mesmo teorema, $\mathcal{E}=(\forall m \varphi) \Rightarrow \varphi\left(\overline{0_{\mathcal{E}}}\right)$ e $\mathcal{E} \models(\forall m \varphi) \Rightarrow \varphi(s(n))$, e por modus ponens obtemos $\mathcal{E} \models \varphi\left(\overline{0_{\mathcal{E}}}\right)$ e $\mathcal{E} \models \varphi(s(n))$.

Agora, $\mathcal{E} \models \varphi(s(n))$ significa $\ulcorner\varphi(s(n))\urcorner=\top_{\mathbb{N}_{\mathcal{E}}}=\chi_{i d_{\mathbb{N}_{\mathcal{E}}}}$, ou seja, $\llbracket \varphi(s(n)) \rrbracket=\left[i d_{\mathbb{N}_{\mathcal{E}}}\right]$, que é o maior elemento de $S u b\left(\mathbb{N}_{\mathcal{E}}\right)$. Assim, $\llbracket \varphi(n) \rrbracket \leq \llbracket \varphi(s(n)) \rrbracket$, e usando o lema 4.6 .7 concluímos que $\mathcal{E}=\varphi(n) \Rightarrow \varphi(s(n))$.

$(\Leftarrow)$ Primeiramente, $\mathcal{E}=\varphi\left(\overline{0_{\mathcal{E}}}\right)$ significa que $\left\ulcorner\varphi\left(\overline{0_{\mathcal{E}}}\right)\right\urcorner=\ulcorner\varphi\urcorner \circ 0_{\mathcal{E}}=\top$. Com isso, lembrando o comentário sobre notação do lema 4.6.12, considere o seguinte diagrama, que é comutativo por 
definição das flechas:

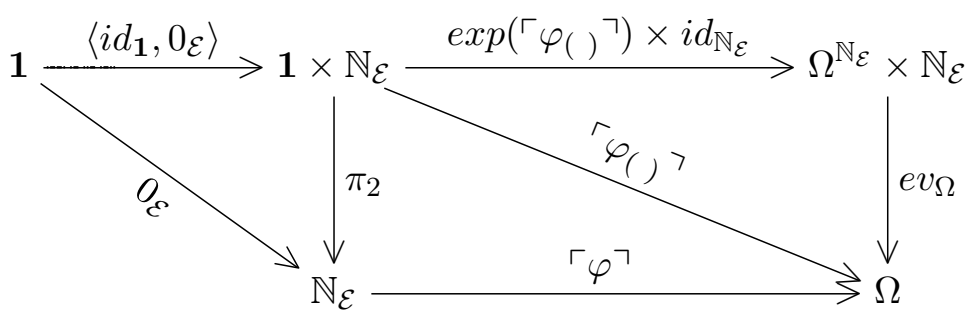

para $\pi_{1}, \pi_{2}$ as projeções do produto $1 \times \mathbb{N}_{\mathcal{E}}$. Agora, considerando a primeira linha do diagrama, note que:

$$
\begin{aligned}
\left.\left\langle\exp \left(\left\ulcorner\varphi_{(}\right)\right\urcorner\right) \circ \pi_{1}, i d_{\mathbb{N}_{\mathcal{E}}} \circ \pi_{2}\right\rangle \circ\left\langle i d_{\mathbf{1}}, 0_{\mathcal{E}}\right\rangle= & \left.\left\langle\exp \left(\left\ulcorner\varphi_{(}\right)\right\urcorner\right) \circ \pi_{1} \circ\left\langle i d_{\mathbf{1}}, 0_{\mathcal{E}}\right\rangle, i d_{\mathbb{N}_{\mathcal{E}}} \circ \pi_{2} \circ\left\langle i d_{\mathbf{1}}, 0_{\mathcal{E}}\right\rangle\right\rangle= \\
& \left.\left.=\left\langle\exp \left(\left\ulcorner\varphi_{(}\right)\right\urcorner\right) \circ i d_{\mathbf{1}}, i d_{\mathbb{N}_{\mathcal{E}}} \circ 0_{\mathcal{E}}\right\rangle=\left\langle\exp \left(\left\ulcorner\varphi_{(}\right)\right\urcorner\right), 0_{\mathcal{E}}\right\rangle
\end{aligned}
$$

Dessa forma, a comutatividade do diagrama garante que:

$$
\left.\left.\left\ulcorner\overline{0_{\mathcal{C}}} \in\left\{n \mid \varphi_{(}\right)\right\}\right\urcorner=\operatorname{ev} \Omega \circ\left\langle\exp \left(\left\ulcorner\varphi_{(}\right)\right\urcorner\right), 0_{\mathcal{E}}\right\rangle=\ulcorner\varphi\urcorner \circ 0_{\mathcal{E}}=\top
$$

donde $\mathcal{E} \models \overline{0_{\mathcal{C}}} \in\{n \mid \varphi\}$ (já simplificando a notação). Um argumento semelhante mostra que a hipótese $\mathcal{E} \models \varphi(n) \Rightarrow \varphi(s(n))$ implica em $\mathcal{E} \models(n \in\{n \mid \varphi\}) \Rightarrow(s(n) \in\{n \mid \varphi\})$, e por generalização universal e modus ponens obtemos $\mathcal{E}=\forall n((n \in\{n \mid \varphi\}) \Rightarrow s(n) \in(\{n \mid \varphi\}))$.

Com isso, a lógica intuicionista também nos fornece $\mathcal{E} \models\left(\overline{0_{\mathcal{C}}} \in\{n \mid \varphi\}\right) \wedge \forall n((n \in\{n \mid$ $\varphi\}) \Rightarrow(s(n) \in\{n \mid \varphi\}))^{5}$. Portanto, como $\left(\mathbb{N}_{\mathcal{C}}, 0_{\mathcal{C}}, s\right)$ é um modelo da aritmética de Peano, de (P4) concluímos que $\llbracket \varphi \rrbracket=\left[i d_{\mathbb{N}_{\mathcal{E}}}\right]$; ou seja, $\ulcorner\varphi\urcorner=\top_{\mathbb{N}_{\mathcal{E}}}$, e $\mathcal{E} \models \varphi$, como queríamos.

Teorema 4.8.4. Sejam $\mathcal{E}$ um topos, $\mathbb{N}_{\mathcal{E}} \in \mathcal{E}_{0}$ e $\mathbb{N}_{\mathcal{E}}, s: \mathbb{N}_{\mathcal{E}} \rightarrow \mathbb{N}_{\mathcal{E}}$ flechas em $\mathcal{E}_{1}$. Então, são equivalentes:

1. $\left(\mathbb{N}_{\mathcal{E}}, 0_{\mathcal{E}}, s\right)$ é um objeto números naturais em $\mathcal{E}$;

2. $\left(\mathbb{N}_{\mathcal{E}}, 0_{\mathcal{E}}, s\right)$ é um modelo da aritmética de Peano em $\mathcal{E}$.

\footnotetext{
${ }^{5}$ Basta usar o axiona (c) e modus ponens duas vezes para mostrar que se $\mathcal{E} \models \psi_{1}$ e $\mathcal{E} \models \psi_{2}$, então $\mathcal{E} \models \psi_{1} \wedge \psi_{2}$.
} 


\section{Capítulo 5}

\section{Modelos Heyting-valorados e topoi}

\section{locálicos}

Nesse capítulo, estudamos a relação entre topoi locálicos e modelos a valores numa álgebra de Heyting. Assim, iniciamos definindo os topoi locálicos e descrevendo algumas de suas propriedades. Em seguida, introduzimos modelos Heyting-valorados, em especial o modelo $V^{(H)}$, e mostramos, então, como podemos obter um topos locálico a partir desse modelo. Feito isso, passamos ao caso booleano para explorar a relação entre o modelo $V^{(B)}$, a relação de forcing da teoria dos conjuntos, e topoi booleanos. Para finalizar, apresentaremos alguns resultados iniciais de um trabalho original em andamento (que pretendemos concluir em [ACM19]) que busca investigar como morfismos entre álgebras de Heyting $H \rightarrow H^{\prime}$ dão origem a morfismos entre os modelos $V^{(H)} \rightarrow V^{\left(H^{\prime}\right)}$ e os topoi correspondentes $\mathbf{S e t}^{(H)} \rightarrow \mathbf{S e t}^{\left(H^{\prime}\right)}$.

No decorrer do capítulo, citamos quais partes específicas das referências foram usadas para cada seção, porém registremos aqui qual foi a bibliografia para o capítulo: [Bel88], [Bel05], [Bor08c], [Gra79b], [Jec03] e [LM94].

\subsection{Topos locálico}

Como o nome sugere, um topos locálico é um topos equivalente ao topos dos feixes sobre um locale. Focamos nesse tipo de topos neste capítulo pois veremos mais adiante que, dada uma álgebra de Heyting completa $H$, conseguimos uma equivalência de categorias entre o modelo $V^{(H)}$ (ou, mais precisamente, um quociente de $V^{(H)}$ ) e a categoria $\mathbf{S h}(H)$ dos feixes sobre 
$H$ (lembrando da proposição 2.2.3 que ser um locale é equivalente a ser uma álgebra de Heyting completa). Nessa seção, apresentamos alguns resultados sobre topoi locálicos, como sua caracterização e algumas propriedades.

Definição 5.1.1. Seja $\mathcal{E}$ um topos. Dizemos que $\mathcal{E}$ é um topos locálico se $\mathcal{E} \simeq \mathbf{S h}(L)$, para algum locale $(L, \leq)$.

Teorema 5.1.2. Seja $\mathcal{E}$ um topos de Grothendieck. Então, são equivalentes:

1. E é um topos locálico;

2. Sub(1) constitui uma família de geradores de $\mathcal{E}$.

Demonstração. A forma mais comum de mostrar a implicação $(2 \Rightarrow 1)$ usa o teorema de Giraud, que não fizemos aqui. Portanto, façamos apenas a implicação $(1 \Rightarrow 2)$; para a recíproca, pode-se consultar o corolário 3.6.4 de [Bor08c] ou o teorema IX.5.1 de [LM94].

$(1 \Rightarrow 2)$ Seja $(L, \leq)$ um locale tal que $\mathcal{E} \simeq \operatorname{Sh}(L)$. Como já foi observado no exemplo 4.7.7, os funtores $L(-, a)$ (para $a \in L$ ) formam uma família geradora. Mostremos que tais funtores correspondem exatamente aos subobjetos de $\mathbf{1}$.

Sejam $F$ um feixe sobre $L$ e $\eta: F \Rightarrow \mathbf{1}$ um mono. Assim, para cada $a \in L, \eta_{a}: F_{0}(a) \longmapsto \mathbf{1}_{0}(a)$ é um mono; e como $\mathbf{1}_{0}(a)=\{*\}$, isso significa que, se $F_{0}(a) \neq \emptyset$, então $F_{0}(a)$ também é unitário. Agora, para todos $a, b \in L$ tais que $a \leq b$, temos:

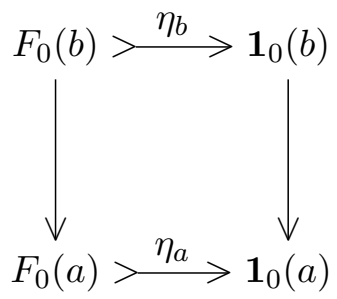

de forma que se $F_{0}(a)=\emptyset$ então $F_{0}(b)=\emptyset$. Isso é exatamente a definição do funtor representável: explicitamente, tomando $a=\bigwedge\left\{b \in L \mid F_{0}(b) \neq \emptyset\right\}, F=L(-, a)$.

Proposição 5.1.3. Seja $\mathcal{E}$ um topos de Grothendieck. Então, são equivalentes:

1. $\mathcal{E}$ é um topos locálico e booleano;

2. $\mathcal{E}$ satisfaz o axioma da escolha; 
3. $\mathcal{E} \simeq \mathbf{S h}(B)$, para alguma álgebra de Boole completa $(B, \leq)$.

Ver corolário 6.10 de [Bel88] ou proposição 7.5.7 de [Bor08c] para a demonstração desse resultado.

Como anunciado inicialmente, façamos agora a demonstração do teorema 2.2.28, agora com uma pequena modificação: consideremos o mesmo funtor Sh : Loc $\rightarrow$ Topos $_{g e o}$ agora com codomínio a categoria dos topoi com morfismos geométricos.

Teorema 5.1.4. O funtor $\mathbf{S h}: \mathbf{L o c} \rightarrow$ Topos $_{g e o}$ é pleno e fiel.

Demonstração. Sejam $(L, \leq),\left(L^{\prime}, \leq^{\prime}\right)$ locales e $\left(\varphi_{*}, \varphi^{*}\right): \mathbf{S h}(L) \rightarrow \mathbf{S h}\left(L^{\prime}\right)$ um morfismo geométrico (ou seja, com $\varphi_{*}: \mathbf{S h}(L) \rightarrow \mathbf{S h}\left(L^{\prime}\right)$ ). Vimos acima que os subobjetos do terminal correspondem aos funtores representáveis $L(-, a)$, que são isomorfos ao próprio $L$. De fato, o isomorfismo é dado por $a \mapsto L(-, a)$, e por Yoneda $N a t(L(-, a), L(-, b)) \cong L(a, b)$. Agora, $\varphi^{*}$ preserva limites finitos por hipótese, logo preserva o objeto terminal e seus subobjetos, e podemos definir a restrição $\varphi_{\mid}^{*}: \operatorname{Sub}\left(\mathbf{1}^{\prime}\right) \rightarrow S u b(\mathbf{1})$, que define uma flecha $f^{*}: L^{\prime} \rightarrow L$; mais especificamente, $f^{*}$ é tal que $\varphi^{*}: L^{\prime}\left(-, a^{\prime}\right) \mapsto L\left(-, f^{*}\left(a^{\prime}\right)\right)$. Além disso, $\varphi^{*}$ preserva colimites arbitrários por possuir adjunto à direita, logo $f^{*}$ também preserva supremos arbitrários (coprodutos em $L$ ) e ínfimos finitos (produtos em $L$ ). Ou seja, seu adjunto à direita $f_{*}: L \rightarrow L^{\prime}$ é um morfismo de locales.

Falta mostrar que, para todo feixe $F$ sobre $L, \mathbf{S h}_{1}\left(f_{*}\right)(F)=F \circ f^{*}:\left(L^{\prime}\right)^{o p} \rightarrow$ Set corresponde a $\varphi_{*}(F)$. Seja $a^{\prime} \in L^{\prime}$, então temos as bijeções naturais:

$$
\begin{aligned}
& \mathbf{S h}_{1}\left(f_{*}\right)(F)\left(a^{\prime}\right)=F\left(f^{*}\left(a^{\prime}\right)\right) \cong \operatorname{Nat}\left(L\left(-, f^{*}\left(a^{\prime}\right)\right), F\right) \\
& \text { (por Yoneda) } \\
& =\operatorname{Nat}\left(\varphi^{*}\left(L\left(-, a^{\prime}\right)\right), F\right) \\
& \text { (pela definição de } f^{*} \text { ) } \\
& \cong \operatorname{Nat}^{\prime}\left(L^{\prime}\left(-, a^{\prime}\right), \varphi_{*}(F)\right) \quad \text { (pois } \varphi^{*} \dashv \varphi_{*} \text { por hipótese) } \\
& \cong \varphi_{*}(F)\left(a^{\prime}\right) \\
& \text { (novamente por Yoneda) }
\end{aligned}
$$

como queríamos.

\subsection{Modelos Heyting-valorados e IZF}

Embora introduzido por D. Scott, R. M. Solovay e P. Vopěnka na década de 1960 para ajudar a compreender o método de forcing de Cohen, o conceito de um modelo a valores booleanos 
é atualmente um conceito mais geral da teoria dos modelos: é uma generalização da noção de estrutura onde os valores verdade são elementos de uma álgebra de Boole completa, não apenas "verdadeiro" e "falso"; analogamente, num modelo Heyting-valorado os valores verdade são elementos de uma álgebra de Heyting completa.

O objetivo dessa seção será fazer uma breve apresentação de modelos a valores numa álgebra de Heyting, em particular do modelo $V^{(H)}$, e indicar como se relacionam com IZF, um modelo intuicionista da teoria dos conjuntos Zermelo-Fraenkel. Uma exposição mais detalhada do caso booleano é feita no capítulo 1 de [Bel05] e no capítulo 14 de [Jec03]; as adaptações para o caso intuicionista são indicadas no capítulo 8 de [Bel05] e em [Gra79b]. Já para uma apresentação detalhada de teoria de conjuntos intuicionista, pode-se consultar [Bel14].

Definição 5.2.1. Seja $\mathcal{L}$ uma linguagem de primeira ordem com igualdade e cujo único símbolo não lógico é $R$, um símbolo relacional binário. Uma $\mathcal{L}$-estrutura Heyting-valorada é uma quádrupla $\mathcal{M}=(M, e q, r, H)$, onde $M$ é uma classe, $(H, \leq)$ uma álgebra de Heyting completa, e $e q, r: M \times M \rightarrow H$ são funções satisfazendo, para todos $m, m^{\prime}, n, n^{\prime} \in M$ :

$$
\begin{array}{ll}
e q(m, m)=1 \quad e q(m, n)=e q(n, m) & e q(m, n) \wedge e q\left(n, n^{\prime}\right) \leq e q\left(m, n^{\prime}\right) \\
r(m, n) \wedge e q\left(m, m^{\prime}\right) \leq r\left(m^{\prime}, n\right) & r(m, n) \wedge e q\left(n, n^{\prime}\right) \leq r\left(m, n^{\prime}\right)
\end{array}
$$

Para toda fórmula $\varphi$ em $\mathcal{L}$ cujas variáveis estão contidas na sequência $\left(x_{1}, \ldots, x_{n}\right)$, definimos a sua $\mathcal{M}$-valoração $\llbracket \varphi \rrbracket^{\mathcal{M}}: M^{n} \rightarrow H$ recursivamente:

- se $\varphi$ é $x_{p}=x_{q}$, então $\llbracket \varphi \rrbracket^{\mathcal{M}}:\left(m_{1}, \ldots, m_{n}\right) \mapsto e q\left(m_{p}, m_{q}\right)$;

- se $\varphi$ é $x_{p} R x_{q}$, então $\llbracket \varphi \rrbracket^{\mathcal{M}}:\left(m_{1}, \ldots, m_{n}\right) \mapsto r\left(m_{p}, m_{q}\right)$;

- se $\varphi$ é $\phi \diamond \psi$, para $\diamond \in\{\wedge, \vee, \rightarrow\}$, então $\llbracket \varphi \rrbracket^{\mathcal{M}}:=\llbracket \phi \rrbracket^{\mathcal{M}} \diamond \llbracket \psi \rrbracket^{\mathcal{M}} ;$

- se $\varphi$ é $\exists y \psi$, então $\llbracket \varphi \rrbracket^{\mathcal{M}}:\left(m_{1}, \ldots, m_{n}\right) \mapsto \bigvee_{m \in M} \llbracket \psi(y \mid t) \rrbracket^{\mathcal{M}}\left(m, m_{1}, \ldots, m_{n}\right)$;

- se $\varphi$ é $\forall y \psi$, então $\llbracket \varphi \rrbracket^{\mathcal{M}}:\left(m_{1}, \ldots, m_{n}\right) \mapsto \bigwedge_{m \in M} \llbracket \psi(y \mid t) \rrbracket^{\mathcal{M}}\left(m, m_{1}, \ldots, m_{n}\right)$.

Com isso, construamos o modelo $V^{(H)}$ da teoria dos conjuntos ${ }^{1}$. Seja $\mathcal{L}_{\text {set }}$ a linguagem da teoria dos conjuntos, uma linguagem de primeira ordem com igualdade e com o símbolo relacional binário $\in$. Abreviamos " $f$ é função" por $F u n(f)$, e, como para flechas em geral, $\operatorname{dom}(f)$ e $\operatorname{cod}(f)$

\footnotetext{
${ }^{1}$ Tecnicamente, $V^{(H)}$ é a classe da definição do modelo $\mathcal{M}=\left(V^{(H)}, e q, r, H\right)$, porém seguimos a notação usual na bibliografia e denotamos o modelo apenas por sua classe.
} 
denotam o domínio e o codomínio de uma função $f$, respectivamente. Além disso, denotamos o universo dos conjuntos por $V$ e a classe dos ordinais por $O r d$.

Definição 5.2.2. Seja $(H, \leq)$ uma álgebra de Heyting completa. Para cada ordinal sucessor $\alpha+1$ e para cada ordinal limite $\lambda$, defina:

$$
\begin{gathered}
V_{0}^{(H)}:=\emptyset \\
V_{\alpha+1}^{(H)}:=\left\{f \mid F u n(f) \text { e } \operatorname{dom}(f) \subseteq V_{\alpha}^{(H)} \text { e } \operatorname{cod}(f) \subseteq H\right\} \\
V_{\lambda}^{(H)}:=\bigcup_{\beta<\lambda} V_{\beta}^{(H)}
\end{gathered}
$$

e, com isso, definimos $V^{(H)}:=\bigcup_{\alpha \in O r d} V_{\alpha}^{(H)}$. Além disso, para cada $x \in V^{(H)}$, definimos a função rank como $\varrho(x):=\min \left\{\alpha \in \operatorname{Ord} \mid \operatorname{dom}(x) \subseteq V_{\alpha}^{(H)}\right\}$. Pode-se verificar, por indução, que para todos $\alpha, \beta \in$ Ord, se $\alpha \leq \beta$ então $V_{\alpha}^{(H)} \subseteq V_{\beta}^{(H)}$.

Agora, definimos a linguagem $\mathcal{L}_{\text {set }}^{(H)}$ adicionando a $\mathcal{L}_{\text {set }}$ uma constante para cada elemento $x \in V^{(H)}$ (que ainda será denotado por $x$ ), e essa nova linguagem nos permite fazer afirmações sobre $V^{(H)}$. Finalmente, então, fazemos recursão (simultânea) na relação bem fundada:

$$
(f, g) \prec(x, y) \Longleftrightarrow(f=x \text { e } g \in \operatorname{dom}(y)) \text { ou }(f \in \operatorname{dom}(x) \text { e } g=y)
$$

para definir o modelo $V^{(H)}$.

Definição 5.2.3. Seja $(H, \leq)$ um álgebra de Heyting completa. Para cada sentença $\sigma$ em $\mathcal{L}_{\text {set }}^{(H)}$, definimos sua $H$-valoração $\llbracket \sigma \rrbracket^{H} \in H$ fazendo:

- sentenças atômicas: sejam $f, g \in V^{(H)}$, então definimos recursivamente:

$$
\begin{gathered}
\llbracket f \in g \rrbracket^{H}:=\bigvee_{y \in \operatorname{dom}(g)}\left(g(y) \wedge \llbracket f=y \rrbracket^{H}\right) \\
\llbracket f=g \rrbracket^{H}:=\bigwedge_{x \in \operatorname{dom}(f)}\left(f(x) \rightarrow \llbracket x \in g \rrbracket^{H}\right) \wedge \bigwedge_{y \in \operatorname{dom}(g)}\left(g(y) \rightarrow \llbracket y \in f \rrbracket^{H}\right)
\end{gathered}
$$

- conectivos: sejam $t, s$ sentenças em $\mathcal{L}_{\text {set }}^{(H)}$, então definimos como nos modelos em geral:

$$
\begin{aligned}
& \llbracket t \wedge s \rrbracket^{H}:=\llbracket t \rrbracket^{H} \wedge \llbracket s \rrbracket^{H} \\
& \llbracket t \vee s \rrbracket^{H}:=\llbracket t \rrbracket^{H} \vee \llbracket s \rrbracket^{H}
\end{aligned}
$$




$$
\llbracket t \rightarrow s \rrbracket^{H}:=\llbracket t \rrbracket^{H} \rightarrow \llbracket s \rrbracket^{H}
$$

- quantificadores: seja $\varphi$ uma fórmula em $\mathcal{L}_{\text {set }}^{(H)}$ com uma variável livre $x$, então novamente definimos como no caso geral:

$$
\begin{aligned}
& \llbracket \exists x \varphi \rrbracket^{H}:=\bigvee_{f \in V^{(H)}} \llbracket \varphi(x \mid f) \rrbracket^{H} \\
& \llbracket \forall x \varphi \rrbracket^{H}:=\bigwedge_{f \in V^{(H)}} \llbracket \varphi(x \mid f) \rrbracket^{H}
\end{aligned}
$$

Definição 5.2.4. Sejam $(H, \leq)$ uma álgebra de Heyting completa e $\sigma$ uma sentença em $\mathcal{L}_{\text {set }}^{(H)}$. Dizemos que $\sigma$ é válida em $V^{(H)}$ se $\llbracket \sigma \rrbracket^{H}=1$. Nesse caso, denotamos $V^{(H)} \models \sigma$.

Teorema 5.2.5. Seja $(H, \leq)$ uma álgebra de Heyting completa. Então, os axiomas da lógica intuicionista de primeira ordem com igualdade são válidos em $V^{(H)}$. Em particular, para todos $u, v, w \in V^{(H)}$ vale:

1. $\llbracket u=u \rrbracket^{H}=1$;

2. se $x \in \operatorname{dom}(u)$, ent $\tilde{a} o \quad u(x) \leq \llbracket x \in u \rrbracket^{H}$;

3. $\llbracket u=v \rrbracket^{H}=\llbracket v=u \rrbracket^{H}$;

4. $\llbracket u=v \rrbracket^{H} \wedge \llbracket v=w \rrbracket^{H} \leq \llbracket u=w \rrbracket^{H}$;

5. $\llbracket u=v \rrbracket^{H} \wedge \llbracket u \in w \rrbracket^{H} \leq \llbracket v \in w \rrbracket^{H} ;$

6. $\llbracket v=w \rrbracket^{H} \wedge \llbracket u \in v \rrbracket^{H} \leq \llbracket u \in w \rrbracket^{H} ;$

7. se $\varphi(x)$ é uma fórmula em $\mathcal{L}_{\text {set }}^{(H)}$, então $\llbracket u=v \rrbracket^{H} \wedge \llbracket \varphi(u) \rrbracket^{H} \leq \llbracket \varphi(v) \rrbracket^{H}$.

Teorema 5.2.6. Seja $(H, \leq)$ uma álgebra de Heyting completa. Então, os axiomas de $\mathbf{I Z \boldsymbol { F }}$ são válidos em $V^{(H)}$ :

1. extensionalidade: $V^{(H)} \models \forall x \forall y(\forall z(z \in x \wedge z \in y) \rightarrow x=y)$;

2. separação: $V^{(H)} \models \forall u \exists v \forall x(x \in v \leftrightarrow(x \in u \wedge \varphi(x)))$, com $v$ não livre na fórmula $\varphi(x)$;

3. coleção: $V^{(H)} \models \forall u(\forall x \in u \exists y \varphi(x, y) \rightarrow \exists v \forall x \in u \exists y \in v \varphi(x, y))$, com $v$ não livre na fórmula $\varphi(x, y)$;

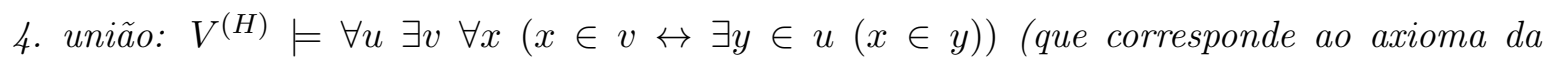
substituição no caso clássico); 
5. conjunto partes: $V^{(H)}=\forall u \exists v \forall x(x \in v \leftrightarrow \forall y \in x(y \in u))$;

6. infinito: $V^{(H)}=\exists u(\emptyset \in u \wedge \forall x \in u \exists y \in u(x \in y))$;

7. regularidade: $V^{(H)} \models \forall x(\forall y \in x \varphi(y) \rightarrow \varphi(x)) \rightarrow \forall x \varphi(x)$, com y não livre em $\varphi(x)$;

8. par: $V^{(H)} \models \forall x \forall y \exists z \forall x(w \in z \leftrightarrow w=x \vee w=y)$.

Teorema 5.2.7. Seja $(B, \leq)$ uma álgebra de Boole completa. Então, os axiomas da lógica clássica de primeira ordem com igualdade são válidos $\mathrm{em} V^{(B)}$ (em particular, as propriedades enunciadas no teorema análogo para álgebras de Heyting novamente valem).

Teorema 5.2.8. Seja $(B, \leq)$ uma álgebra de Boole completa. Então, os axiomas de $\boldsymbol{Z F C}$ são válidos em $V^{(B)}$. Os axiomas são os mesmos de IZF (embora possamos omitir o axioma do par), e adicionamos o axioma da escolha (por exemplo, na forma do lema de Zorn).

Pode-se consultar os teoremas 1.17 e 1.33 de [Bel05] para a demonstração desses resultados no caso booleano e a seção 2.2 de [Gra79b] para o caso intuicionista.

\section{$5.3 V^{(H)}$ e descrições equivalentes de $\operatorname{Sh}(H)$}

Para o restante desta seção, fixamos $(H, \leq)$ uma álgebra de Heyting completa.

Definição 5.3.1. Considere a relação de equivalência em $V^{(H)}$ dada por $f \equiv g$ se, e somente se, $\llbracket f=g \rrbracket^{H}=1$. Assim, definimos a categoria Set $^{(H)}$ fazendo:

$$
\begin{gathered}
\left(\operatorname{Set}^{(H)}\right)_{0}:=\frac{V^{(H)}}{\equiv} \\
\operatorname{Set}^{(H)}([x],[y]):=\left\{[\phi] \in\left(\operatorname{Set}^{(H)}\right)_{0} \mid \llbracket \phi: x \rightarrow y \text { é função } \rrbracket^{(H)}=1\right\}
\end{gathered}
$$

As flechas não dependem da escolha dos representantes de $[x]$ e $[y]$. A composição e a identidade são dadas como em Set.

Definição 5.3.2. Um $H$-set é um par $(X, \delta)$ onde $X$ é um conjunto e $\delta: X \times X \rightarrow H$ satisfazendo, para todos $x, y, z \in X$ :

1. $\delta(x, y)=\delta(y, x)$;

2. $\delta(x, y) \wedge \delta(y, z) \leq \delta(x, z)$. 
Definição 5.3.3. Seja $(X, \delta),\left(X^{\prime}, \delta^{\prime}\right) H$-sets. Um morfismo de $H$-sets $\phi:(X, \delta) \rightarrow\left(X^{\prime}, \delta^{\prime}\right)$ é uma função $\phi: X \times X^{\prime} \rightarrow H$ satisfazendo, para todos $x, y \in X$ e $x^{\prime}, y^{\prime} \in X^{\prime}$ :

1. $\delta^{\prime}\left(x^{\prime}, y^{\prime}\right) \wedge \phi\left(x, y^{\prime}\right) \leq \phi\left(x, x^{\prime}\right)$

2. $\delta(x, y) \wedge \phi\left(x, y^{\prime}\right) \leq \phi\left(y, y^{\prime}\right)$

3. $\phi\left(x, x^{\prime}\right) \wedge \phi\left(x, y^{\prime}\right) \leq \delta^{\prime}\left(x^{\prime}, y^{\prime}\right)$;

4. $\bigvee_{z^{\prime} \in X^{\prime}} \phi\left(x, z^{\prime}\right)=\delta(x, x)$.

Ou seja, um morfismo pode ser entendido como uma "relação funcional com valores em $H$ ".

Agora, dados morfismos de $H$-sets $\phi:(X, \delta) \rightarrow\left(X^{\prime}, \delta^{\prime}\right)$ e $\psi:\left(X^{\prime}, \delta^{\prime}\right) \rightarrow\left(X^{\prime \prime}, \delta^{\prime \prime}\right)$, podemos definir sua composição $\psi \circ \phi:(X, \delta) \rightarrow\left(X^{\prime \prime}, \delta^{\prime \prime}\right)$ fazendo, para todos $x \in X, x^{\prime \prime} \in X^{\prime \prime}$ :

$$
(\psi \circ \phi)\left(x, x^{\prime \prime}\right)=\bigvee_{x^{\prime} \in X^{\prime}} \phi\left(x, x^{\prime}\right) \wedge \psi\left(x^{\prime}, x^{\prime \prime}\right)
$$

e a identidade de $i d_{(X, \delta)}:(X, \delta) \rightarrow(X, \delta)$ é a função $i d_{(X, \delta)}: X \times X \rightarrow H$ tal que

$$
i d_{(X, \delta)}(x, y)=\delta(x, y), \text { para todos } x, y \in X
$$

Portanto, podemos definir a categoria $H$-Set dos $H$-sets e seus morfismos.

Um resultado sobre morfismos de $H$-sets que será importante mais adiante é:

Proposição 5.3.4. Sejam $\phi, \psi:(X, \delta) \rightarrow\left(X^{\prime}, \delta^{\prime}\right)$ morfismos de $H$-sets. Então, são equivalentes:

1. $\phi=\psi ;$

2. $\phi\left(x, x^{\prime}\right) \leq \psi\left(x, x^{\prime}\right)$, para todos $x \in X$ e $x^{\prime} \in X^{\prime}$.

Ver lema 2.8.6 de [Bor08c] para a demonstração.

Definição 5.3.5. Seja $(X, \delta)$ um $H$-set. Um unitário de $(X, \delta)$ é uma função $\sigma: X \rightarrow H$ satisfazendo, para todos $x, y \in X$ :

1. $\sigma(x) \wedge \sigma(y) \leq \delta(x, y)$;

2. $\sigma(x) \wedge \delta(x, y) \leq \sigma(y)$. 
Note que, dado $x \in X$, a função $\sigma_{x}: X \rightarrow H$ tal que $\sigma_{x}(y)=\delta(x, y)$, para todo $y \in H$, define um unitário.

Definição 5.3.6. Seja $(X, \delta)$ um $H$-set. Considere $\sigma(X)$ o conjunto dos unitários de $(X, \delta)$. Dizemos que $(X, \delta)$ é completo se a função $\Upsilon: X \rightarrow \sigma(X)$, dada por $\Upsilon(x)=\sigma_{x}$, para todo $x \in X$, é bijetora. Denotamos por $\mathbf{c} H$-Set $\subseteq H$-Set a subcategoria (plena) dos $H$-sets completos.

Existe também uma descrição alternativa dos $H$-sets completos:

Proposição 5.3.7. A categoria $\mathbf{c} H$-Set é isomorfa à categoria que tem como objetos $H$-sets completos e como flechas $f:(X, \delta) \rightarrow\left(X^{\prime}, \delta^{\prime}\right)$ as funções $f: X \rightarrow X^{\prime}$ satisfazendo, para todos $x, y \in X:$

1. $\delta(x, y) \leq \delta^{\prime}(f(x), f(y))$;

2. $\delta(x, x)=\delta^{\prime}(f(x), f(x))$.

A composição é dada simplesmente pela composição de funções (e a identidade é a função identidade).

Para obter um morfismo de $H$-sets $\phi:(X, \delta) \rightarrow\left(X^{\prime}, \delta^{\prime}\right)$ a partir de $f: X \rightarrow X^{\prime}$ acima, basta definir $\phi: X \times X^{\prime} \rightarrow H$ fazendo $\phi\left(x, x^{\prime}\right)=\delta^{\prime}\left(f(x), x^{\prime}\right)$, para todos $x \in X, x^{\prime} \in X^{\prime}$.

Teorema 5.3.8. Seja $(X, \delta)$ um $H$-set. Podemos definir o $H$-set $(\sigma(X), \sigma(\delta)) \operatorname{com} \sigma(\delta): \sigma(X) \times$ $\sigma(X) \rightarrow H$ dada por:

$$
\sigma(\delta)(\rho, \tau)=\bigvee_{x \in X} \rho(x) \wedge \tau(x), \text { para todos }(\rho, \tau) \in \sigma(X) \times \sigma(X)
$$

Então, $(\sigma(X), \sigma(\delta))$ é completo e é isomorfo a $(X, \delta)$.

Os isomorfismos inversos $\phi:(X, \delta) \rightarrow(\sigma(X), \sigma(\delta))$ e $\psi:((\sigma(X), \sigma(\delta)) \rightarrow(X, \delta)$ são funções $\phi: X \times \sigma(X) \rightarrow H, \psi: \sigma(X) \times X \rightarrow H$ dadas por:

$$
\begin{aligned}
& \phi(x, \rho)=\rho(x), \text { para todos }(x, \rho) \in X \times \sigma(X) \\
& \psi(\rho, x)=\rho(x), \text { para todos }(\rho, x) \in \sigma(X) \times X
\end{aligned}
$$

Pode-se consultar a demonstração completa no teorema 2.9.5 de [Bor08c].

Corolário 5.3.9. Temos a equivalência de categorias $H$-Set $\simeq \mathbf{c H}$-Set. 
Com isso, podemos definir o funtor $\Gamma: \mathbf{S h}(H) \rightarrow \mathbf{c} H$-Set fazendo:

$$
\begin{aligned}
& \Gamma_{0}(F)=\left(X_{F}, \delta_{F}\right) \text {, para todo feixe } F \text { sobre } H, \operatorname{com} X_{F}:=\coprod_{a \in H} F_{0}(a) \text { e } \\
& \qquad \delta_{F}: X_{F} \times X_{F} \rightarrow H \text { é dada por }((s, b),(t, c)) \mapsto \bigvee\left\{d \leq b \wedge c|s|_{d}^{b}=\left.t\right|_{d} ^{c}\right\}
\end{aligned}
$$

$\Gamma_{1}(\eta): \Gamma_{0}(F) \rightarrow \Gamma_{0}(G)$, para toda $\eta \in \mathbf{S h}(H)_{1}$, onde

$$
\Gamma_{1}(\eta)(s, b)=\left(\eta_{b}(s), b\right), \text { para todo }(s, b) \in \Gamma_{0}(F)
$$

(usando a descrição equivalente de morfismo de $H$-sets completos)

Teorema 5.3.10. O funtor $\Gamma: \mathbf{S h}(H) \rightarrow \mathbf{c H}$-Set definido acima é pleno e fiel, e para todo $H$-set completo $(X, \delta)$ existe um feixe $F$ em $\mathbf{S h}(H)$ tal que $(X, \delta) \cong \Gamma_{0}(F)$. Ou seja, $\Gamma$ define uma equivalência de categorias $\mathbf{S h}(H) \simeq \mathbf{c} H$-Set.

A demonstração desse resultado é feita detalhadamente no teorema 2.9.8 de [Bor08c].

Finalmente, para mostrar a equivalência entre $H$-Set e Set $^{(H)}$, precisamos de duas noções em $V^{(H)}$. Primeiramente, dado $x \in V$, definimos $\hat{x}$ um "representante natural" de $x$ em $V^{(H)}$ através de recursão na relação bem fundada $y \in X: \hat{x}:=\{(\hat{y}, 1) \mid y \in x\}$. Além disso, definimos o par ordenado em $V^{(H)}$ fazendo, para todos $u, v \in V^{(H)}$,

$$
\begin{gathered}
\{u\}^{(H)}:=\{(u, 1)\} \\
\{u, v\}^{(H)}:=\{u\}^{(H)} \cup\{v\}^{(H)} \\
(u, v)^{(H)}=\left\{\{u\}^{(H)},\{u, v\}^{(H)}\right\}^{(H)}
\end{gathered}
$$

Agora, seja $(X, \delta)$ um $H$-set. Para cada $x \in X$, defina $\dot{x} \in V^{(H)}$ fazendo

$$
\operatorname{dom}(\dot{x}):=\{\hat{z} \mid z \in X\} \quad \text { e } \quad \dot{x}(\hat{z}):=\delta(x, z)
$$

para todo $z \in X$. Com isso, seja $X^{\dagger} \in V^{(H)}$ dado por, para todo $x \in X$,

$$
\operatorname{dom}\left(X^{\dagger}\right):=\{\dot{x} \mid x \in X\} \quad \text { e } \quad X^{\dagger}(\dot{x}):=\delta(x, x)
$$

Dado um morfismo de $H$-sets $\phi:(X, \delta) \rightarrow\left(X^{\prime}, \delta^{\prime}\right)$, defina $\phi^{\dagger} \in V^{(H)}$ tomando

$$
\operatorname{dom}\left(\phi^{\dagger}\right):=\left\{\left(\dot{x}, \dot{x}^{\prime}\right)^{(H)} \mid x \in X, x^{\prime} \in X^{\prime}\right\}
$$




$$
\phi^{\dagger}\left(\left(\dot{x}, \dot{x}^{\prime}\right)^{(H)}\right)=\phi\left(x, x^{\prime}\right), \text { para todos } x \in X, x^{\prime} \in X^{\prime}
$$

Como $V^{(H)} \models F u n\left(\phi^{\dagger}\right)$, podemos definir um funtor $\Phi: H$-Set $\rightarrow \operatorname{Set}^{(H)}$ fazendo $\Phi_{0}(X, \delta)=$ $\left[X^{\dagger}\right]$, para todo $H$-set $(X, \delta)$, e $\Phi_{1}(\phi)=\phi^{\dagger}$, para toda $\phi \in H$-Set . $_{1}$.

Por outro lado, dado $u \in V^{(H)}$, defina $X_{u}:=\operatorname{dom}(u)$ e $\delta_{u}: X_{u} \times X_{u} \rightarrow H$ como

$$
\delta_{u}(x, y):=\llbracket x \in u \rrbracket^{H} \wedge \llbracket x=y \rrbracket^{H} \wedge \llbracket y \in u \rrbracket^{H}, \text { para todos } x, y \in X_{u}
$$

Note, porém, que $\llbracket u=u^{\prime} \rrbracket^{H}=1$ não implica em $X_{u}=\operatorname{dom}(u)=\operatorname{dom}\left(u^{\prime}\right)=X_{u^{\prime}}$, e que não podemos simplesmente definir um $H$-set usando $[\operatorname{dom}(u)]$ pois essa classe não é conjunto (veremos, mais adiante, que $\left\{u^{\prime} \in V^{(H)} \mid \llbracket u=u^{\prime} \rrbracket^{H}=1\right\}$ é classe própria). Assim, para definir um funtor $\Psi:$ Set $^{(H)} \rightarrow H$-Set, usaremos um truque de Scott.

Primeiramente, se $\llbracket u=u^{\prime} \rrbracket^{H}=1$, então $\left(X_{u}, \delta_{u}\right) \cong\left(X_{u^{\prime}}, \delta_{u^{\prime}}\right)$. De fato, defina $\lambda_{u, u^{\prime}}$ : $\left(X_{u}, \delta_{u}\right) \rightarrow\left(X_{u^{\prime}}, \delta_{u^{\prime}}\right)$ como a função $\lambda_{u, u^{\prime}}: X_{u} \times X_{u^{\prime}} \rightarrow H$ tal que

$$
\lambda_{u, u^{\prime}}\left(x, x^{\prime}\right):=\llbracket x \in u \rrbracket^{H} \wedge \llbracket x=x^{\prime} \rrbracket^{H} \wedge \llbracket x^{\prime} \in u^{\prime} \rrbracket^{H}, \operatorname{para} \operatorname{todos} x \in \operatorname{dom}(u), x^{\prime} \in \operatorname{dom}\left(u^{\prime}\right)
$$

Verifiquemos que isso define um morfismo de $H$-sets. Sejam $x, y \in X_{u}$ e $x^{\prime}, y^{\prime} \in X_{u^{\prime}}$.

1. $\delta_{u^{\prime}}\left(x^{\prime}, y^{\prime}\right) \wedge \lambda_{u, u^{\prime}}\left(x, y^{\prime}\right) \leq \lambda_{u, u^{\prime}}\left(x, x^{\prime}\right)$. De fato,

$$
\begin{aligned}
\delta_{u^{\prime}}\left(x^{\prime}, y^{\prime}\right) & \wedge \lambda_{u, u^{\prime}}\left(x, y^{\prime}\right)= \\
& =\llbracket x^{\prime} \in u^{\prime} \rrbracket^{H} \wedge \llbracket x^{\prime}=y^{\prime} \rrbracket^{H} \wedge \llbracket y^{\prime} \in u^{\prime} \rrbracket^{H} \wedge \llbracket x \in u \rrbracket^{H} \wedge \llbracket x=y^{\prime} \rrbracket^{H} \wedge \llbracket y^{\prime} \in u^{\prime} \rrbracket^{H} \\
& \leq \llbracket x^{\prime} \in u^{\prime} \rrbracket^{H} \wedge \llbracket x^{\prime}=y^{\prime} \rrbracket^{H} \wedge \llbracket x \in u \rrbracket^{H} \wedge \llbracket x=y^{\prime} \rrbracket^{H} \\
& \leq \llbracket x \in u \rrbracket^{H} \wedge \llbracket x=x^{\prime} \rrbracket^{H} \wedge \llbracket x^{\prime} \in u^{\prime} \rrbracket^{H} \\
& =\lambda_{u, u^{\prime}}\left(x, x^{\prime}\right)
\end{aligned}
$$

2. $\delta_{u}(x, y) \wedge \lambda_{u, u^{\prime}}\left(x, y^{\prime}\right) \leq \lambda_{u, u^{\prime}}\left(y, y^{\prime}\right)$. De fato,

$$
\begin{aligned}
\delta_{u}(x, y) & \wedge \lambda_{u, u^{\prime}}\left(x, y^{\prime}\right)= \\
& =\llbracket x \in u \rrbracket^{H} \wedge \llbracket x=y \rrbracket^{H} \wedge \llbracket y \in u \rrbracket^{H} \wedge \llbracket x \in u \rrbracket^{H} \wedge \llbracket x=y^{\prime} \rrbracket^{H} \wedge \llbracket y^{\prime} \in u^{\prime} \rrbracket^{H} \\
& \leq \llbracket x=y \rrbracket^{H} \wedge \llbracket y \in u \rrbracket^{H} \wedge \llbracket x=y^{\prime} \rrbracket^{H} \wedge \llbracket y^{\prime} \in u^{\prime} \rrbracket^{H} \\
& \leq \llbracket y \in u \rrbracket^{H} \wedge \llbracket y=y^{\prime} \rrbracket^{H} \wedge \llbracket y^{\prime} \in u^{\prime} \rrbracket^{H} \\
& =\lambda_{u, u^{\prime}}\left(y, y^{\prime}\right)
\end{aligned}
$$


3. $\lambda_{u, u^{\prime}}\left(x, x^{\prime}\right) \wedge \lambda_{u, u^{\prime}}\left(x, y^{\prime}\right) \leq \delta_{u^{\prime}}\left(x^{\prime}, y^{\prime}\right)$. De fato,

$$
\begin{aligned}
\lambda_{u, u^{\prime}}\left(x, x^{\prime}\right) & \wedge \lambda_{u, u^{\prime}}\left(x, y^{\prime}\right)= \\
& =\llbracket x \in u \rrbracket^{H} \wedge \llbracket x=x^{\prime} \rrbracket^{H} \wedge \llbracket x^{\prime} \in u^{\prime} \rrbracket^{H} \wedge \llbracket x \in u \rrbracket^{H} \wedge \llbracket x=y^{\prime} \rrbracket^{H} \wedge \llbracket y^{\prime} \in u^{\prime} \rrbracket^{H} \\
& \leq \llbracket x=x^{\prime} \rrbracket^{H} \wedge \llbracket x^{\prime} \in u^{\prime} \rrbracket^{H} \wedge \llbracket x=y^{\prime} \rrbracket^{H} \wedge \llbracket y^{\prime} \in u^{\prime} \rrbracket^{H} \\
& \leq \llbracket x^{\prime} \in u^{\prime} \rrbracket^{H} \wedge \llbracket x^{\prime}=y^{\prime} \rrbracket^{H} \wedge \llbracket y^{\prime} \in u^{\prime} \rrbracket^{H} \\
& =\delta_{u^{\prime}}\left(x^{\prime}, y^{\prime}\right)
\end{aligned}
$$

4. $\bigvee_{z^{\prime} \in X_{u^{\prime}}} \lambda_{u, u^{\prime}}\left(x, z^{\prime}\right)=\delta_{u}(x, x)$. De fato, usando que $\delta_{u}(x, x)=\llbracket x \in u \rrbracket^{H}$,

- por um lado, para todo $z^{\prime} \in X_{u^{\prime}}$ temos:

$$
\lambda_{u, u^{\prime}}\left(x, z^{\prime}\right)=\llbracket x \in u \rrbracket^{H} \wedge \llbracket x=z^{\prime} \rrbracket^{H} \wedge \llbracket z^{\prime} \in u^{\prime} \rrbracket^{H} \leq \llbracket x \in u \rrbracket^{H}
$$

Logo, $\bigvee_{z^{\prime} \in X_{u^{\prime}}} \lambda_{u, u^{\prime}}\left(x, z^{\prime}\right) \leq \delta_{u}(x, x)$;

- por outro lado, para todo $z \in X_{u^{\prime}}$ vale

$$
\begin{aligned}
u^{\prime}\left(z^{\prime}\right) \wedge \llbracket z^{\prime}= & x \rrbracket^{H}=\llbracket x=z^{\prime} \rrbracket^{H} \wedge u^{\prime}\left(z^{\prime}\right) \wedge \llbracket z^{\prime}=z^{\prime} \rrbracket^{H} \leq \\
& \leq \llbracket x=z^{\prime} \rrbracket^{H} \wedge\left(\bigvee_{t^{\prime} \in X_{u^{\prime}}} u^{\prime}\left(t^{\prime}\right) \wedge \llbracket t^{\prime}=z^{\prime} \rrbracket^{H}\right)=\llbracket x=z^{\prime} \rrbracket^{H} \wedge \llbracket z^{\prime} \in u^{\prime} \rrbracket^{H}
\end{aligned}
$$

Portanto,

$$
\llbracket x \in u^{\prime} \rrbracket^{H}=\bigvee_{z^{\prime} \in X_{u^{\prime}}} u^{\prime}\left(z^{\prime}\right) \wedge \llbracket z^{\prime}=x \rrbracket^{H} \leq \bigvee_{z^{\prime} \in X_{u^{\prime}}} \llbracket x=z^{\prime} \rrbracket^{H} \wedge \llbracket z^{\prime} \in u^{\prime} \rrbracket^{H}
$$

Mas observe que $\llbracket u=u^{\prime} \rrbracket^{H}=1$ implica em $\llbracket x \in u \rrbracket^{H}=\llbracket x \in u^{\prime} \rrbracket^{H}$, logo:

$$
\begin{aligned}
\llbracket x \in u \rrbracket^{H} & =\llbracket x \in u \rrbracket^{H} \wedge \llbracket x \in u^{\prime} \rrbracket^{H} \\
& \leq \llbracket x \in u \rrbracket^{H} \wedge\left(\bigvee_{z^{\prime} \in X_{u^{\prime}}} \llbracket x=z^{\prime} \rrbracket^{H} \wedge \llbracket z^{\prime} \in u^{\prime} \rrbracket^{H}\right) \\
& =\bigvee_{z^{\prime} \in X_{u^{\prime}}} \llbracket x \in u \rrbracket^{H} \wedge \llbracket x=z^{\prime} \rrbracket^{H} \wedge \llbracket z^{\prime} \in u^{\prime} \rrbracket^{H}
\end{aligned}
$$

Ou seja, $\delta_{u}(x, x) \leq \bigvee_{z^{\prime} \in X_{u^{\prime}}} \lambda_{u, u^{\prime}}\left(x, z^{\prime}\right)$

Finalmente, verifiquemos que $\lambda_{u, u^{\prime}}$ é um isomorfismo, com morfismo inverso $\lambda_{u, u^{\prime}}^{-1}=\lambda_{u^{\prime}, u}$ : 
$\left(X_{u^{\prime}}, \delta_{u^{\prime}}\right) \rightarrow\left(X_{u}, \delta_{u}\right)$ (onde, naturalmente, $\left.\lambda_{u^{\prime}, u}\left(x^{\prime}, x\right)=\lambda_{u, u^{\prime}}\left(x, x^{\prime}\right)\right)$. Para todos $x, y \in X_{u}$, temos:

$$
\begin{aligned}
& \left(\lambda_{u^{\prime}, u} \circ \lambda_{u, u^{\prime}}\right)(x, y)=\bigvee_{x^{\prime} \in X_{u^{\prime}}} \lambda_{u, u^{\prime}}\left(x, x^{\prime}\right) \wedge \lambda_{u^{\prime}, u}\left(x^{\prime}, y\right)= \\
& =\bigvee_{x^{\prime} \in X_{u^{\prime}}} \llbracket x \in u \rrbracket^{H} \wedge \llbracket x=x^{\prime} \rrbracket^{H} \wedge \llbracket x^{\prime} \in u^{\prime} \rrbracket^{H} \wedge \llbracket y \in u \rrbracket^{H} \wedge \llbracket y=x^{\prime} \rrbracket^{H} \wedge \llbracket x^{\prime} \in u^{\prime} \rrbracket^{H} \leq \\
& \quad \leq \llbracket x \in u \rrbracket^{H} \wedge \llbracket x=y \rrbracket^{H} \wedge \llbracket y \in u \rrbracket^{H}=\delta_{u}(x, y)
\end{aligned}
$$

Portanto, usando a proposição 5.3.4, concluímos que $\lambda_{u^{\prime}, u} \circ \lambda_{u, u^{\prime}}=i d_{(X, \delta)}$. De forma análoga, pode-se verificar a igualdade $\lambda_{u, u^{\prime}} \circ \lambda_{u^{\prime}, u}=i d_{\left(X^{\prime}, \delta^{\prime}\right)}$.

Agora, para cada $[u] \in \operatorname{Set}^{(H)}$, seja $I^{[u]}$ a categoria com

$$
I_{0}^{[u]}:=[u]_{m} \quad I_{1}^{[u]}:=[u]_{m} \times[u]_{m}
$$

onde $[u]_{m}$ é a classe de equivalência dos elementos com rank mínimo. Defina o funtor $F^{[u]}$ : $I^{[u]} \rightarrow H$-Set fazendo:

$$
\begin{gathered}
F_{0}^{[u]}\left(u^{\prime}\right):=\left(X_{u}, \delta_{u}\right), \text { para todo } u^{\prime} \in[u]_{m} \\
F_{1}^{[u]}\left(u^{\prime}, u^{\prime \prime}\right):=\lambda_{u^{\prime}, u^{\prime \prime}}:\left(X_{u^{\prime}}, \delta_{u^{\prime}}\right) \rightarrow\left(X_{u^{\prime \prime}}, \delta_{u^{\prime \prime}}\right), \text { para todos } u^{\prime}, u^{\prime \prime} \in[u]_{m}
\end{gathered}
$$

Enfim, podemos definir o funtor $\Psi$ : Set $^{(H)} \rightarrow H$-Set fazendo $\Psi_{0}([u])=\lim _{u^{\prime} \in[u]_{m}} F^{[u]}\left(u^{\prime}\right)$.

Podemos obter uma descrição mais explícita desse funtor. O produto de uma família $\left\{\left(X_{i}, \delta_{i}\right) \mid i \in I\right\}$ em $H$-Set é dado por $(P, \delta)$, sendo o conjunto apenas o produto cartesiano $P=\prod_{i \in I} X_{i}$ e $\delta: P \times P \rightarrow H$ dada por:

$$
\delta\left(\left(x_{i}\right)_{i \in I},\left(x_{i}^{\prime}\right)_{i \in I}\right)=\bigwedge_{i \in I} \delta\left(x, x^{\prime}\right)
$$

As projeções $\pi_{j}: P \times X_{j} \rightarrow H$ do produto, por sua vez, são dadas por:

$$
\pi_{j}\left(\left(x_{i}\right)_{i \in I}, x_{j}^{\prime}\right)=\delta_{j}\left(x_{j}, x_{j}^{\prime}\right)
$$

para cada $j \in I$ (ver exercício 2.13.15 de [Bor08c]). Ademais, o equalizador de dois morfismos $\phi, \psi:(X, \delta) \rightarrow\left(X^{\prime}, \delta^{\prime}\right)$ de $H$-sets é $(X, \tau)$, onde

$$
\tau(x, y)=\bigvee_{x^{\prime} \in X^{\prime}} \phi\left(x, x^{\prime}\right) \wedge \psi\left(y, x^{\prime}\right)
$$


(ver exercício 2.13.16 de [Bor08c]).

Finalmente, podemos usar a construção de um limite a partir de produtos e equalizadores (enunciada em 1.5.11, mas feita com detalhes no teorema 2.8.1 de [Bor08a]), onde denotamos $\Psi([u])$ por $\lim F^{[u]}$ :

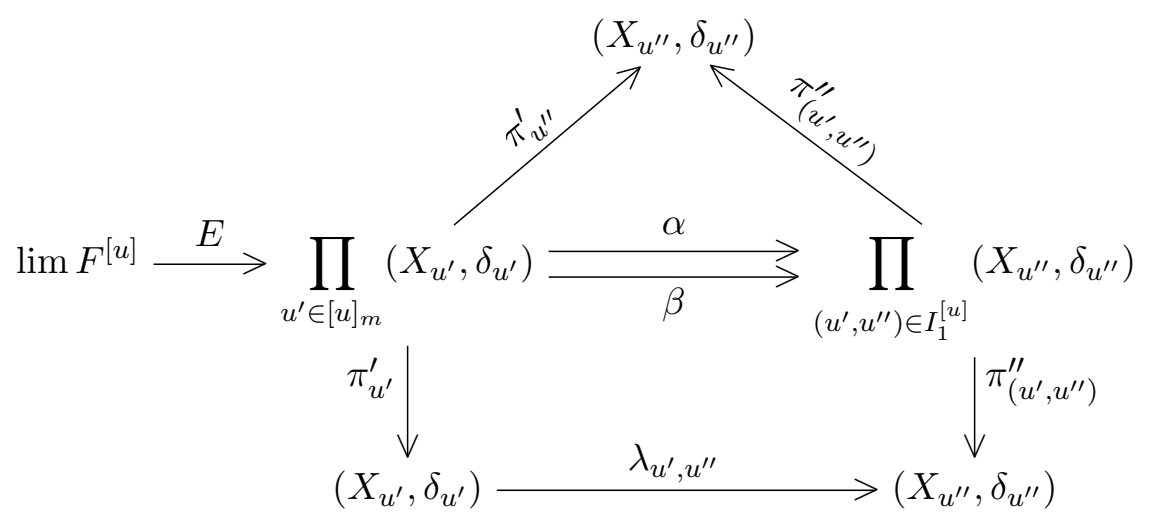

onde $\pi^{\prime}, \pi^{\prime \prime}$ denotam as projeções dos produtos e $(\lim F, E)$ é o equalizador de $\alpha$ e $\beta$, que são os morfismos que tornam o diagrama comutativo, isto é:

$$
\pi_{\left(u^{\prime}, u^{\prime \prime}\right)}^{\prime \prime} \circ \alpha=\pi_{u^{\prime \prime}}^{\prime} \quad \pi_{\left(u^{\prime}, u^{\prime \prime}\right)}^{\prime \prime} \circ \beta=\lambda_{u^{\prime}, u^{\prime \prime}} \circ \pi_{u^{\prime}}^{\prime}
$$

Explicitamente, $\alpha$ e $\beta$ satisfazem, para todos $\vec{x}=\left(x_{u^{\prime}}\right)_{u^{\prime} \in[u]_{m}}\left(\operatorname{com} x_{u^{\prime}} \in X_{u^{\prime}}\right)$ e $x^{\prime \prime} \in X_{u^{\prime \prime}}$ :

$$
\begin{aligned}
\bigvee_{\vec{y} \in \operatorname{cod}(\alpha)} \alpha(\vec{x}, \vec{y}) \wedge \delta_{u^{\prime \prime}}\left(y_{\left(u^{\prime}, u^{\prime \prime}\right)}, x^{\prime \prime}\right) & =\left(\pi_{\left(u^{\prime}, u^{\prime \prime}\right)}^{\prime \prime} \circ \alpha\right)\left(\vec{x}, x^{\prime \prime}\right)=\pi_{u^{\prime \prime}}^{\prime}\left(\vec{x}, x^{\prime \prime}\right)=\delta_{u^{\prime \prime}}\left(x_{u^{\prime \prime}}, x^{\prime \prime}\right) \\
\bigvee_{\vec{y} \in \operatorname{cod}(\alpha)} \beta(\vec{x}, \vec{y}) \wedge \delta_{u^{\prime \prime}}\left(y_{\left(u^{\prime}, u^{\prime \prime}\right)}, x^{\prime \prime}\right) & =\left(\pi_{\left(u^{\prime}, u^{\prime \prime}\right)}^{\prime \prime} \circ \beta\right)\left(\vec{x}, x^{\prime \prime}\right)= \\
= & \left(\lambda_{u^{\prime}, u^{\prime \prime}} \circ \pi_{u^{\prime}}^{\prime}\right)\left(\vec{x}, x^{\prime \prime}\right)=\bigvee_{x^{\prime} \in X_{u^{\prime}}} \delta_{u^{\prime}}\left(x_{u^{\prime}}, x^{\prime}\right) \wedge \lambda_{u^{\prime}, u^{\prime \prime}}\left(x^{\prime}, x^{\prime \prime}\right)
\end{aligned}
$$

Pode-se proceder de forma semelhante para as flechas. Para cada $f \in V^{(H)}$ tal que $\llbracket f: u \rightarrow$ $v$ é função $\rrbracket^{H}=1$, defina $\lambda_{f}:\left(X_{u}, \delta_{u}\right) \rightarrow\left(X_{v}, \delta_{v}\right)$ como:

$$
\lambda_{f}(x, y)=\llbracket x \in u \rrbracket^{H} \wedge \llbracket(x, y) \in f \rrbracket^{H} \wedge \llbracket y \in v \rrbracket^{H}
$$

Agora, dada $f^{\prime} \in V^{(H)}$ tal que $\llbracket f^{\prime}: u^{\prime} \rightarrow v^{\prime}$ é função $\rrbracket^{H}=1$ e $\llbracket f=f^{\prime} \rrbracket^{H}=1$ (o que já implica 
em $u \equiv u^{\prime}$ e $\left.v \equiv v^{\prime}\right)$, obtemos o seguinte diagrama comutativo:

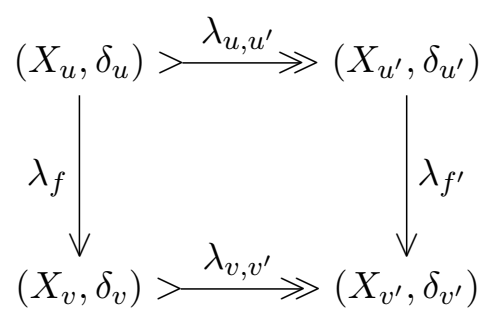

Portanto, podemos construir uma flecha $\Psi_{1}([f]): \lim _{u^{\prime} \in[u]_{m}} F\left(u^{\prime}\right) \rightarrow \lim _{v^{\prime} \in[v]_{m}} F\left(v^{\prime}\right)$.

Teorema 5.3.11. Os funtores $\Phi, \Psi$ definidos acima constituem uma equivalência de categorias $H-\operatorname{Set} \simeq \operatorname{Set}^{(H)}$.

Essas construções, com algumas modificações, são feitas no final do apêndice de [Bel05].

\subsection{Forcing, modelos a valores booleanos e topoi booleanos}

Tendo agora as definições iniciais de modelos a valores booleanos, mostremos como a semântica em $V^{(B)}$ se relaciona com o forcing de Cohen e com a topologia da dupla negação num topos.

Definição 5.4.1. Seja $(P, \leq)$ um conjunto parcialmente ordenado. Para cada $p \in P$, defina:

$$
\mathcal{O}_{p}:=\{q \in P \mid q \leq p\}
$$

Com isso, defina $\mathcal{O}(P)$ como a topologia sobre $P$ gerada por $\left\{\mathcal{O}_{p} \mid p \in P\right\}$ e considere a álgebra de Heyting dada por $(H, \leq):=(\mathcal{O}(P), \subseteq)$. Então, definimos a relação de forcing intuicionista $\| \vdash$ entre um elemento $p \in P$ e uma sentença $\sigma$ de $\mathcal{L}_{\text {set }}^{(H)}$ fazendo:

$$
p \| \vdash \sigma \quad \text { se, e somente se } \quad \mathcal{O}_{p} \leq \llbracket \sigma \rrbracket^{H}
$$

Observação. Dados $a_{1}, \ldots, a_{n}, b_{1}, \ldots, b_{n} \in V^{(H)}$, denote $\bar{a}:=\left(a_{1}, \ldots, a_{n}\right), \bar{b}:=\left(b_{1}, \ldots, b_{n}\right) \mathrm{e}$

$$
\llbracket \bar{a}=\bar{b} \rrbracket^{H}:=\llbracket a_{1}=b_{1} \rrbracket^{H} \wedge \ldots \wedge \llbracket a_{n}=b_{n} \rrbracket^{H}
$$

Assim, suponha que $p \| \vdash \varphi(\bar{a})$, isto é, $\mathcal{O}_{p} \leq \llbracket \varphi(\bar{a}) \rrbracket^{H}$. Note que faz sentido considerar a relação de forcing no quociente: $p \| \Vdash \varphi([\bar{a}])$, pois independe da escolha do representante. De fato, como $\llbracket \bar{a}=\bar{b} \rrbracket^{H} \wedge \llbracket \varphi(\bar{a}) \rrbracket^{H}=\llbracket \bar{a}=\bar{b} \rrbracket^{H} \wedge \llbracket \varphi(\bar{b}) \rrbracket^{H}$, se $\llbracket \bar{a}=\bar{b} \rrbracket^{H}=1$, então $\llbracket \varphi(\bar{a}) \rrbracket^{H}=\llbracket \varphi(\bar{b}) \rrbracket^{H}$. 
Proposição 5.4.2. Sejam $(P, \leq)$ um conjunto parcialmente ordenado, $p \in P$ e $(H, \leq)$ como na definição acima. Então, para todas sentenças $\sigma, \tau$ de $\mathcal{L}_{\text {set }}^{(H)}$ e toda fórmula $\varphi$ em $\mathcal{L}_{\text {set }}^{(H)}$ com uma variável livre $x$, temos:

1. $V^{(H)} \models \sigma$ se, e somente se, para todo $q \in P, q \Vdash \sigma$;

2. se $p \| \Vdash \sigma$, então para todo $q \in \mathcal{O}_{p}$ vale $q \| \vdash \sigma$;

3. $p \| \vdash \sigma \wedge \tau$ se, e somente se, $p \| \vdash \sigma$ e $p \| \vdash \tau$;

4. $p \| \vdash \sigma \vee \tau$ se, e somente se, $p \| \vdash \sigma$ ou $p \| \vdash \tau$;

5. $p \Vdash \Vdash \sigma \rightarrow \tau$ se, e somente se, para todo $q \in \mathcal{O}_{p}$ vale que $q \Vdash \vdash \sigma$ implica em $q \Vdash \leftarrow$;

6. $p \| \vdash \neg \sigma$ se, e somente se, para todo $q \in \mathcal{O}_{p}$ não vale $q \| \vdash \sigma$;

7. $p \Vdash \Vdash \exists x \varphi$ se, e somente se, $p \| \vdash \varphi(u)$, para algum $u \in V^{(H)}$;

8. $p \| \Vdash \forall x \varphi$ se, e somente se, $p \| \vdash \varphi(u)$, para todo $u \in V^{(H)}$.

Para o caso booleano, usa-se a noção de completamento booleano. Pode-se mostrar, usando os abertos regulares da topologia $\mathcal{O}(P)$ (que formam uma álgebra de Boole), que todo conjunto parcialmente ordenado $(P, \leq)$ possui um completamento booleano $(B, e)$ (único a menos de isomorfismo), com $(B, \leq)$ uma álgebra de Boole e $e: P \rightarrow D$ sobrejetora, onde $D \subseteq B$ é um subconjunto denso, satisfazendo:

$$
\text { existe } r \in P \text { tal que } r \leq p \text { e } r \leq q \quad \text { se, e somente se } \quad e(p) \wedge e(q) \neq 0
$$

para todos $p, q \in P .^{2}$

Definição 5.4.3. Seja $(P, \leq)$ um conjunto parcialmente ordenado. Considere seu completamento booleano $(B, e)$. Então, definimos a relação de forcing fraca $\Vdash_{w}$ entre um elemento $p \in P$ e uma sentença $\sigma$ de $\mathcal{L}_{\text {set }}^{(H)}$ fazendo:

$$
p \Vdash_{w} \sigma \quad \text { se, e somente se } \quad e(p) \leq \llbracket \sigma \rrbracket^{B}
$$

Proposição 5.4.4. Sejam $(P, \leq)$ um conjunto parcialmente ordenado, $p \in P e(B, \leq)$ como na definição acima. Então, para todas sentenças $\sigma, \tau$ de $\mathcal{L}_{\text {set }}^{(B)}$ e toda fórmula $\varphi$ em $\mathcal{L}_{\text {set }}^{(H)}$ com uma variável livre $x$, temos:

\footnotetext{
${ }^{2}$ Em particular, mostra-se que um conjunto parcialmente ordenado $(P, \leq)$ é refinado se, e somente se, $\mathcal{O}_{p}$ é aberto regular, para todo $p \in P$, e que o mapa $e: p \mapsto \mathcal{O}_{p}$ é um isomorfismo e satisfaz o desejado. Essas construções são feitas detalhadamente na primeira seção do capítulo 2 de [Bel05] e no capítulo 14 de [Jec03].
} 
1. $V^{(B)} \models \sigma$ se, e somente se, para todo $q \in P, q \Vdash_{w} \sigma$;

2. se $p \Vdash_{w} \sigma$, então para todo $q \in \mathcal{O}_{p}$ vale $q \Vdash_{w} \sigma$;

3. se $p \Vdash_{w} \sigma$, então não vale $p \Vdash_{w} \neg \sigma$;

4. existe $q \in \mathcal{O}_{p}$ tal que $q \Vdash_{w} \sigma$ ou $q \Vdash_{w} \neg \sigma$;

5. $p \Vdash_{w} \sigma \wedge \tau$ se, e somente se, $p \Vdash_{w} \sigma$ e $p \Vdash_{w} \tau$;

6. $p \Vdash_{w} \sigma \vee \tau$ se, e somente se, para todo $q \in \mathcal{O}_{p}$, existe $r \in \mathcal{O}_{q}$ tal que $r \Vdash_{w} \sigma$ ou $r \Vdash_{w} \tau$;

7. $p \Vdash_{w} \sigma \rightarrow \tau$ se, e somente se, para todo $q \in \mathcal{O}_{p}$ vale que $q \Vdash_{w} \sigma$ implica em $q \Vdash_{w} \tau$;

8. $p \Vdash_{w} \neg \sigma$ se, e somente se, para todo $q \in \mathcal{O}_{p}$ não vale $q \Vdash_{w} \sigma$;

9. $p \Vdash_{w} \exists x \varphi$ se, e somente se, para todo $q \in \mathcal{O}_{p}$, existem $r \in \mathcal{O}_{q}$ e $u \in V^{(B)}$ tais que $r \Vdash_{w} \varphi(u)$;

10. $p \Vdash_{w} \forall x \varphi$ se, e somente se, $p \Vdash_{w} \varphi(u)$, para todo $u \in V^{(B)}$.

A noção original de forcing, a relação de forcing forte (ou de Cohen), denotada $\Vdash_{s}$, está relacionada com o forcing fraco acima através da equivalência:

$$
p \Vdash_{w} \sigma \quad \text { se, e somente se } \quad p \Vdash_{s} \neg \neg \sigma
$$

A diferença principal entre as noções de forcing fraca e forte é que a primeira satisfaz as leis da lógica clássica enquanto a segunda satisfaz as leis da lógica intuicionista, e a relação acima entre $\Vdash_{w}$ e $\Vdash_{s}$ corresponde à tradução de Glivenko-Gödel-Gentzen entre as lógicas clássica e intuicionista. Aliás, a única diferença entre o forcing forte e o intuicionista é que, na proposição 5.4.2, o último item deve ser substituído por:

$$
p \Vdash_{s} \forall x \varphi \quad \text { se, e somente se, } \quad p \Vdash_{s} \neg \exists x \neg \varphi
$$

Como é amplamente conhecido, a noção de forcing foi primeiramente introduzida e usada por P. Cohen para mostrar a independência da hipótese do contínuo da teoria de conjuntos $\mathbf{Z F}$. A ideia do método é estender um modelo $M$ de $\mathbf{Z F}$ a um modelo $M[G]$ de $\mathbf{Z F}$ juntando a $M$ um conjunto "genérico" $G$ (mais precisamente, um subconjunto de um conjunto parcialmente ordenado satisfazendo certas condições); pode-se mostrar, então, que dada uma sentença $\sigma$, $M[G] \models \sigma$ se, e somente se, existe $p \in G$ tal que $p \Vdash_{w} \sigma$. 
Para mostrar a independência da hipótese do contínuo, em particular, a extensão do modelo é construída usando genéricos do conjunto parcialmente ordenado $\left(P_{c}, \leq_{c}\right)$ dado por

$$
P_{c}=\left\{p: a \rightarrow b \mid a \subseteq \omega_{2} \times \omega \text { finito e } b \subseteq\{0,1\}\right\}
$$

$p \leq_{c} q \quad$ se, e somente se, $\quad q \subseteq p$ (como conjuntos de pares ordenados)

de forma que o novo modelo possui uma função injetora $\omega_{2} \longmapsto\{0,1\}^{\omega}$. Ou seja, nesse modelo a hipótese do contínuo falha. ${ }^{3}$

Uma construção semelhante pode ser feita num topos, como exposto na seção VI.2 de [LM94]. Primeiro, considera-se o conjunto parcialmente ordenado $\left(P_{c}, \leq_{c}\right)$ munido da topologia densa $J_{d}$ (na qual os crivos são densos), que é uma topologia de Grothendieck. Então, chamando de $\mathbf{P}$ o topos de Grothendieck $\mathbf{P}:=\mathbf{S h}\left(P_{c}, J_{d}\right)$, usa-se a topologia da dupla negação para definir o topos de Cohen $\mathcal{C}:=\mathbf{S h}(\mathbf{P}, \neg \neg)$. Assim, pode-se mostrar que no topos de Cohen, existem um objeto $K$ e monos $\mathbb{N}_{\mathcal{C}} \longmapsto K \longmapsto \Omega^{\mathbb{N}_{\mathcal{C}}}$ (com $\mathbb{N}_{\mathcal{C}}$ o objeto números naturais e $\Omega$ o classificador de subobjetos de $\mathcal{C}$ ), e não existem epis $\mathbb{N}_{\mathcal{C}} \rightarrow K$ e $K \rightarrow \Omega^{\mathbb{N}_{\mathcal{C}}}$.

\subsection{Morfismos induzidos em modelos Heyting-valorados}

Apesar de termos a conexão "horizontal" entre modelos e topoi:

$$
H \rightsquigarrow V^{(H)} \rightsquigarrow \operatorname{Set}^{(H)} \simeq H \text {-Set }
$$

a conexão "vertical" entre flechas $H \rightarrow H^{\prime}, V^{(H)} \rightarrow V^{(H)}$, etc. não parece ter sido muito explorada na literatura; o único caso considerado foi o de monomorfismos completos entre álgebras de Boole completas (ver exercício 3.12 de [Bel05]) e retrações associadas a eles (capítulo 3 de [Gui13]). Isso nos motivou a estudar como poderíamos induzir flechas nos modelos a partir de flechas mais gerais entre álgebras de Heyting completas: com inspiração na equivalência dos modelos com topoi, consideramos funções que se comportam como o adjunto à esquerda de um morfismo de locales.

No restante da seção, $(H, \leq),\left(H^{\prime}, \leq^{\prime}\right)$ serão álgebras de Heyting completas e $f: H \rightarrow H^{\prime}$ será uma função que preserva supremos arbitrários e ínfimos finitos.

\footnotetext{
${ }^{3}$ Para mais detalhes dessa demonstração, ver, por exemplo, teorema 14.32 de [Jec03].
} 
Definição 5.5.1. (primeira proposta) Definimos recursivamente uma família

$$
\left\{\tilde{f}_{\alpha}: V_{\alpha}^{(H)} \rightarrow V_{\alpha}^{\left(H^{\prime}\right)} \mid \alpha \in O r d\right\}
$$

onde $\rightarrow$ indica que $\tilde{f}_{\alpha}$ são "semifunções" (isto é, para todo $x \in V_{\alpha}^{(H)}$, existe $x^{\prime} \in V_{\alpha}^{\left(H^{\prime}\right)}$ tal que $\left.\left(x, x^{\prime}\right) \in \tilde{f}_{\alpha}\right)$, da seguinte forma: para cada $\alpha \in \operatorname{Ord}$ e todo $\left(x, x^{\prime}\right) \in V_{\alpha}^{(H)} \times V^{\left(H^{\prime}\right)},\left(x, x^{\prime}\right) \in \tilde{f}_{\alpha}$ se, e somente se, existe $\varepsilon: \operatorname{dom}(x) \rightarrow \operatorname{dom}\left(x^{\prime}\right)$ sobrejetora tal que $(u, \varepsilon(u)) \in \tilde{f}_{\varrho(x)}$ para todo $u \in \operatorname{dom}(x)$, e o diagrama abaixo é comutativo:

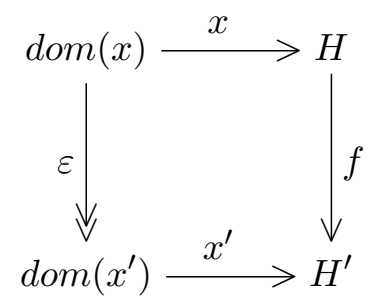

Nessas condições, dizemos que $\varepsilon$ testemunha $\left(x, x^{\prime}\right) \in \tilde{f}_{\alpha}$. Note que, se supusermos (por indução) que $\tilde{f}_{\beta}$ esteja definida para todo ordinal $\beta<\alpha$, então a semifunção $\tilde{f}_{\varrho(x)}: V_{\varrho(x)}^{(H)} \rightarrow V_{\varrho(x)}^{\left(H^{\prime}\right)}$ está definida, $\operatorname{logo} \operatorname{dom}\left(x^{\prime}\right) \subseteq V_{\varrho(x)}^{\left(H^{\prime}\right)}$ e $x^{\prime} \in V_{\alpha}^{\left(H^{\prime}\right)}{ }^{4}$

Com isso, definimos $\tilde{f}:=\bigcup_{\alpha \in O r d} \tilde{f}_{\alpha}$ e

$$
\operatorname{dom}(\tilde{f})=\bigcup_{\alpha \in O r d} \operatorname{dom}\left(\tilde{f}_{\alpha}\right)=\bigcup_{\alpha \in O r d} V_{\alpha}^{(H)}=V^{(H)}
$$

logo $\tilde{f}$ também é semifunção $\tilde{f}: V^{(H)} \rightarrow V^{(H)}$.

Proposição 5.5.2. Se $f$ é injetora, então, para todo $\alpha \in O r d, \tilde{f}_{\alpha}$ é função e é injetora.

Demonstração. Suponha, por indução, que $\tilde{f}_{\beta}$ seja função injetora, para todo $\beta<\alpha$, e seja $\left(x, x^{\prime}\right) \in \tilde{f}_{\alpha}$. Então, $\varepsilon=\tilde{f}_{\varrho(x) \mid}: \operatorname{dom}(x) \rightarrow \operatorname{dom}\left(x^{\prime}\right)$ é uma bijeção, pois já é sobrejetora por definição, e, como $(u, \varepsilon(u)) \in \tilde{f}_{\varrho(x)}$ para todo $u \in \operatorname{dom}(x)$, a hipótese de indução nos fornece que $\varepsilon=\tilde{f}_{\varrho(x) \mid}$ é injetora. Portanto, usando o diagrama comutativo da definição, $x^{\prime}$ está unicamente determinado por $x^{\prime}=f \circ x \circ \varepsilon^{-1}$, ou seja, $\tilde{f}_{\alpha}$ é função. Além disso, se $x \neq y\left(\right.$ em $\left.V_{\alpha}^{(H)}\right)$, então o fato de $f$ ser injetora implica em

$$
\tilde{f}_{\alpha}(x)=f \circ x \circ \varepsilon^{-1} \neq f \circ y \circ \varepsilon^{-1}=\tilde{f}_{\alpha}(y)
$$

\footnotetext{
${ }^{4}$ Observe que, pela definição de $V_{0}^{(H)}, \tilde{f}_{0}$ é a função vazia, logo nas provas por indução omitiremos o caso inicial $\alpha=0$ pois é trivial.
} 
$\operatorname{logo} \tilde{f}_{\alpha}$ é injetora.

Essa função, portanto, cobre o resultado enunciado no exercício 3.12 de [Bel05].

Não obstante, observe que a definição que apresentamos possui um grave problema: não podemos garantir que os domínios das semifunções $\tilde{f}_{\alpha}$ sejam $V_{\alpha}^{(H)}$, para cada $\alpha \in O r d$. Por exemplo, considere as álgebras de Boole $\mathbf{2}=\{0,1\}$ e $\mathbf{4}=\{0, a, \neg a, 1\}(\operatorname{com} 0 \neq a \neq 1)$ e a função $f: \mathbf{4} \rightarrow \mathbf{2}$ dada por $f(a)=0$ e $f(\neg a)=1$. Primeiramente, note que:

$$
\begin{array}{cc}
V_{0}^{(\mathbf{4})}=\emptyset & V_{0}^{(\mathbf{2})}=\emptyset \\
V_{1}^{(\mathbf{4})}=\{\emptyset\} & V_{1}^{(\mathbf{2})}=\{\emptyset\} \\
V_{2}^{(\mathbf{4})}=\{\{(\emptyset, 0)\},\{(\emptyset, 1)\},\{(\emptyset, a)\},\{(\emptyset, \neg a)\}\} & V_{2}^{(\mathbf{2})}=\{\{(\emptyset, 0)\},\{(\emptyset, 1)\}\}
\end{array}
$$

Seja $x:=\{((\emptyset, 0), 0)\},((\emptyset, a), 1)\}$ e chame $u:=\{(\emptyset, 0)\}$ e $v:=\{(\emptyset, 1)\}$. É fácil verificar que $\{(\emptyset, 0)\}$ é único elemento de $V_{2}^{(\mathbf{2})}$ tal que $(u,\{(\emptyset, 0)\}),(v,\{(\emptyset, 0)\}) \in \tilde{f}_{2}$.

Com isso, considere $x^{\prime} \in V^{(\mathbf{2})}$ e suponha que exista $\varepsilon: \operatorname{dom}(x) \rightarrow \operatorname{dom}\left(x^{\prime}\right)$ tal que $(u, \varepsilon(u)),(v, \varepsilon(v)) \in \tilde{f}_{2}$, ou seja, $\varepsilon(u)=\varepsilon(v)=\{(\emptyset, 0)\}$. Mas nesse caso, não podemos garantir a comutatividade do diagrama, pois teríamos:

$$
0=\varphi(x(u))=x^{\prime}(\varepsilon(u))=x^{\prime}(\varepsilon(v))=\varphi(x(v))=1
$$

Logo, não existe nenhum $x^{\prime} \in V^{(\mathbf{2})}$ tal que $\left(x, x^{\prime}\right) \in \tilde{f}_{3}$.

Para resolver esse problema, acrescentamos mais elementos à imagem da semifunção, fazendo o fechamento pela relação de equivalência $\equiv$ (outra possibilidade seria fechar as imagens apenas para rank mínimo).

Alternativamente, poderíamos ainda supor que $\left(x, x^{\prime}\right) \in \tilde{f}_{\alpha}$ se, e somente se, existe testemunha $\varepsilon: \operatorname{dom}(x) \rightarrow \operatorname{dom}\left(x^{\prime}\right)$ como na definição original, e, para todo $u \in \operatorname{dom}(x)$, existe $u^{\prime} \in V^{\left(H^{\prime}\right)}$ tal que $\left(u, u^{\prime}\right) \in \tilde{f}_{\varrho(x)}$ e $\llbracket \varepsilon(u)=u^{\prime} \rrbracket^{H^{\prime}}=1^{\prime}$.

Escolhemos a primeira condição que mencionamos acima para a definição final, porém todas são equivalentes se passarmos ao quociente por $\equiv$.

Definição 5.5.1. (complemento) Acrescentando às condições da definição do início da seção, também supomos que se $\left(x, x^{\prime}\right) \in \tilde{f}_{\alpha}$ (ou seja, se existe uma testemunha para isso) e $\llbracket x^{\prime}=$ $y^{\prime} \rrbracket^{H^{\prime}}=1^{\prime}$, então $\left(x, y^{\prime}\right) \in \tilde{f}_{\alpha}$. 
Observação. Note que $V^{(H)}$ é classe própria (para $H \neq\{0\}$ ), pois existe uma injeção $V \longmapsto V^{(H)}$. Assim, pode-se mostrar que, para todo $x \in V^{(H)},\left\{y \in V^{(H)} \mid \llbracket x=y \rrbracket^{H}=1\right\}$ é classe própria. De fato, para todo $\Sigma \subseteq V^{(H)} \operatorname{com} \Sigma \cap \operatorname{dom}(x)=\emptyset$, podemos definir $y_{\Sigma}: \operatorname{dom}(x) \cup \Sigma \rightarrow H$ fazendo:

$$
y_{\Sigma}(u)= \begin{cases}x(u) & , \text { se } u \in \operatorname{dom}(x) \\ 0 & , \text { se } u \in \Sigma\end{cases}
$$

de forma que $\llbracket x=y_{\Sigma} \rrbracket^{H}=1$.

Finalmente, podemos mostrar que $\tilde{f}_{\alpha}$ realmente possui o domínio que queremos, e o argumento é semelhante ao usado para demonstrar o caso injetor que fizemos acima com a proposta inicial de definição.

Proposição 5.5.3. Para todo $\alpha \in O r d, \operatorname{dom}\left(\tilde{f}_{\alpha}\right)=V_{\alpha}^{(H)}$ (e a imagem de $\tilde{f}_{\alpha}$ é fechada por $\left.\equiv\right)$.

Demonstração. Façamos indução: suponha que, para todo $\beta \in \operatorname{Ord} \operatorname{com} \beta<\alpha, \operatorname{dom}\left(\tilde{f}_{\beta}\right)=V_{\beta}^{(H)}$. Seja $x \in V_{\alpha}^{(H)}$, e note que, para todo $u \in \operatorname{dom}(x) \subseteq V_{\varrho(x)}^{(H)}$, a imagem $\tilde{f}_{\varrho(x)}(\{u\})$ é classe própria, pois é não vazia e fechada por $\equiv$ (por definição). Assim, usando o axioma da substituição, podemos definir uma injeção $\varepsilon: \operatorname{dom}(x) \longmapsto V^{\left(H^{\prime}\right)}$ satisfazendo $(u, \varepsilon(u)) \in \tilde{f}_{\varrho(x)}$, que pode ser restringida a uma bijeção $\tau: \operatorname{dom}(x) \rightarrow \varepsilon(\operatorname{dom}(x))$. Com isso, basta tomar $x^{\prime} \in V^{\left(H^{\prime}\right)}$ como $x^{\prime}: \varepsilon(\operatorname{dom}(x)) \rightarrow H^{\prime}$ dada por $x^{\prime}:=f \circ x \circ \tau^{-1}$, de forma que $\left(x, x^{\prime}\right) \in \tilde{f}_{\alpha}$ e, pois, $\operatorname{dom}\left(\tilde{f}_{\alpha}\right)=V_{\alpha}^{(H)}$.

Note, portanto, que com essa nova definição para todo $x \in V_{\alpha}^{(H)}$ existe $x^{\prime} \in V^{\left(H^{\prime}\right)}$ tal que $\left(x, x^{\prime}\right) \in \tilde{f}_{\alpha}$ é testemunhado por uma bijeção $\tau: \operatorname{dom}(x) \rightarrow \operatorname{dom}\left(x^{\prime}\right)$ (não apenas uma sobrejeção). Inclusive, na definição de $\tilde{f}_{\alpha}$ poderíamos supor a existência de testemunha bijetora, e novamente isso seria equivalente às outras possíveis definições ao passar ao quociente.

Teorema 5.5.4. Para todos $\left(x, x^{\prime}\right),\left(y, y^{\prime}\right),\left(z, z^{\prime}\right) \in \tilde{f}$, vale:

$$
f\left(\llbracket y \in x \rrbracket^{H}\right) \leq^{\prime} \llbracket y^{\prime} \in x^{\prime} \rrbracket^{H^{\prime}} \quad e \quad f\left(\llbracket x=z \rrbracket^{H}\right) \leq^{\prime} \llbracket x^{\prime}=z^{\prime} \rrbracket^{H^{\prime}}
$$

Demonstração. A demonstração é feita por indução na relação bem fundada

$$
(u, x) \prec(v, y) \Longleftrightarrow(u=v \text { e } x \in \operatorname{dom}(y)) \text { ou }(u \in \operatorname{dom}(v) \text { e } x=y)
$$


Seja $\varepsilon: \operatorname{dom}(x) \rightarrow \operatorname{dom}\left(x^{\prime}\right)$ satisfazendo as condições da definição 5.5.1. Então:

$$
\begin{array}{rlr}
f\left(\llbracket y \in x \rrbracket^{H}\right) & =f\left(\bigvee_{u \in \operatorname{dom}(x)} x(u) \wedge \llbracket u=y \rrbracket^{H}\right) & \text { (por definição) } \\
& =\bigvee_{u \in d o m(x)}^{\prime} f(x(u)) \wedge^{\prime} f\left(\llbracket u=y \rrbracket^{H}\right) & (\text { pois } f \text { preserva } \wedge, \bigvee) \\
& \leq \bigvee_{u \in d o m(x)}^{\prime} f(x(u)) \wedge^{\prime} \llbracket \varepsilon(u)=y^{\prime} \rrbracket^{H} & (\text { por hipótese de indução e }(u, \varepsilon(u)) \in \tilde{f})) \\
& =\bigvee_{u \in d o m(x)}^{\prime} x^{\prime}(\varepsilon(u)) \wedge^{\prime} \llbracket \varepsilon(u)=y^{\prime} \rrbracket^{H} & \text { (usando que } \left.\left(x, x^{\prime}\right),(u, \varepsilon(u)) \in \tilde{f}\right) \\
& =\bigvee_{u^{\prime} \in \operatorname{dom}\left(x^{\prime}\right)}^{\prime} x^{\prime}\left(u^{\prime}\right) \wedge^{\prime} \llbracket u^{\prime}=y^{\prime} \rrbracket^{H} & \text { (pois } \varepsilon \text { é sobrejetora) } \\
& =\llbracket y^{\prime} \in x^{\prime} \rrbracket^{H^{\prime}} & \text { (por definição) }
\end{array}
$$

Agora, como $(H, \leq)$ é álgebra de Heyting, note que $f$ preservar ínfimos finitos implica em $f$ ser crescente, e também implica em $f(a \rightarrow b) \leq f(a) \rightarrow f(b)$, para todos $a, b \in H$. Com isso, seja $\tau: \operatorname{dom}(z) \rightarrow \operatorname{dom}\left(z^{\prime}\right)$ satisfazendo as condições da definição 5.5.1. Então:

$$
\begin{aligned}
& f\left(\llbracket x=z \rrbracket^{H}\right)= \\
& =f\left(\bigwedge_{\substack{u \in \operatorname{dom}(x) \\
v \in \operatorname{dom}(z)}}\left(x(u) \rightarrow \llbracket u \in z \rrbracket^{H}\right) \wedge\left(z(v) \rightarrow \llbracket v \in x \rrbracket^{H}\right)\right) \\
& \leq \bigwedge_{u \in \operatorname{dom}(x)} f\left(x(u) \rightarrow \llbracket u \in z \rrbracket^{H}\right) \wedge^{\prime} f\left(z(v) \rightarrow \llbracket v \in x \rrbracket^{H}\right) \quad \text { (pois } f \text { é crescente) } \\
& v \in \operatorname{dom}(z) \\
& \leq \bigwedge_{\substack{u \in \operatorname{dom}(x) \\
v \in \operatorname{dom}(z)}}^{\prime}\left(f(x(u)) \rightarrow^{\prime} f\left(\llbracket u \in z \rrbracket^{H}\right)\right) \wedge^{\prime}\left(f(z(v)) \rightarrow^{\prime} f\left(\llbracket v \in x \rrbracket^{H}\right)\right) \quad \text { (comentários acima) } \\
& \leq \bigwedge_{\substack{u \in \operatorname{dom}(x) \\
v \in \operatorname{dom}(z)}}^{\prime}\left(f(x(u)) \rightarrow^{\prime} \llbracket \varepsilon(u) \in z^{\prime} \rrbracket^{H^{\prime}}\right) \wedge^{\prime}\left(f(z(v)) \rightarrow^{\prime} \llbracket \tau(v) \in x^{\prime} \rrbracket^{H^{\prime}}\right) \quad \text { (comentários abaixo) } \\
& \left.=\bigwedge_{\substack{u \in \operatorname{dom}(x) \\
v \in \operatorname{dom}(z)}}^{\prime}\left(x^{\prime}(\varepsilon(u)) \rightarrow^{\prime} \llbracket \varepsilon(u) \in z^{\prime} \rrbracket^{H^{\prime}}\right) \wedge^{\prime}\left(z^{\prime}(\tau(v)) \rightarrow^{\prime} \llbracket \tau(v) \in x^{\prime} \rrbracket^{H^{\prime}}\right) \quad \text { (elementos de } \tilde{f}\right) \\
& =\bigwedge_{\substack{u^{\prime} \in \operatorname{dom}\left(x^{\prime}\right) \\
v^{\prime} \in \operatorname{dom}\left(z^{\prime}\right)}}^{\prime}\left(x^{\prime}\left(u^{\prime}\right) \rightarrow^{\prime} \llbracket u^{\prime} \in z^{\prime} \rrbracket^{H^{\prime}}\right) \wedge^{\prime}\left(z^{\prime}\left(v^{\prime}\right) \rightarrow^{\prime} \llbracket v^{\prime} \in x^{\prime} \rrbracket^{H^{\prime}}\right) \quad \text { (pois } \varepsilon, \tau \text { são sobre) } \\
& =\llbracket x^{\prime}=z^{\prime} \rrbracket^{H^{\prime}} \\
& \text { (por definição) }
\end{aligned}
$$


No quarto passo, usamos que a implicação é crescente na segunda coordenada, e que a hipótese de indução nos fornece as desigualdades:

$$
f\left(\llbracket u \in z \rrbracket^{H}\right) \leq \llbracket \varepsilon(u) \in z^{\prime} \rrbracket^{H^{\prime}} \quad \text { e } \quad f\left(\llbracket v \in x \rrbracket^{H}\right) \leq \llbracket \tau(v) \in x^{\prime} \rrbracket^{H^{\prime}}
$$

Esse resultado se estende facilmente às fórmulas positivas (apenas com $\wedge, \vee$ ) com quantificadores restritos (da forma $\exists u \in x$ e $\forall u \in x$ ), usando que o corolário 1.18 de [Bel05] nos fornece:

$$
\begin{aligned}
& \llbracket \exists u \in x \varphi(u) \rrbracket^{H}=\bigvee_{u \in \operatorname{dom}(x)} x(u) \wedge \llbracket \varphi(u) \rrbracket^{H} \\
& \llbracket \forall u \in x \varphi(u) \rrbracket^{H}=\bigwedge_{u \in \operatorname{dom}(x)} x(u) \rightarrow \llbracket \varphi(u) \rrbracket^{H}
\end{aligned}
$$

Corolário 5.5.5. Seja $\varphi$ uma fórmula positiva com quantificadores restritos. Então, para todos $\left(a_{1}, a_{1}^{\prime}\right), \ldots,\left(a_{n}, a_{n}^{\prime}\right) \in \tilde{f}$, vale:

$$
f\left(\llbracket \varphi\left(a_{1}, \ldots, a_{n}\right) \rrbracket^{H}\right) \leq^{\prime} \llbracket \varphi\left(a_{1}^{\prime}, \ldots, a_{n}^{\prime}\right) \rrbracket^{H^{\prime}}
$$

Demonstração. A prova é por indução na complexidade. O caso das sentenças atômicas é feito no teorema anterior, e os casos com a disjunção e a conjunção são imediatos do fato de $f$ preservar supremos e ínfimos finitos. Para os quantificadores, a demonstração é semelhante ao teorema anterior. Seja $\left(x, x^{\prime}\right) \in \tilde{f} \operatorname{com}$ testemunha $\varepsilon: \operatorname{dom}(x) \rightarrow \operatorname{dom}\left(x^{\prime}\right)$. Então:

$$
\begin{aligned}
& f\left(\llbracket \exists u \in x \varphi(u) \rrbracket^{H}\right)=f\left(\bigvee_{u \in \operatorname{dom}(x)} x(u) \wedge \llbracket \varphi(u) \rrbracket^{H}\right) \\
& =\bigvee_{u \in \operatorname{dom}(x)}^{\prime} f(x(u)) \wedge^{\prime} f\left(\llbracket \varphi(u) \rrbracket^{H}\right) \\
& \left.\leq \bigvee_{u \in \operatorname{dom}(x)}^{\prime} f(x(u)) \wedge^{\prime} \llbracket \varphi(\varepsilon(u)) \rrbracket^{H} \quad(\text { hipótese de indução e }(u, \varepsilon(u)) \in \tilde{f})\right) \\
& =\bigvee_{u \in \operatorname{dom}(x)}^{\prime} x^{\prime}(\varepsilon(u)) \wedge^{\prime} \llbracket \varphi(\varepsilon(u)) \rrbracket^{H} \quad\left(\text { usando que }\left(x, x^{\prime}\right),(u, \varepsilon(u)) \in \tilde{f}\right) \\
& =\bigvee_{u^{\prime} \in \operatorname{dom}\left(x^{\prime}\right)}^{\prime} x^{\prime}\left(u^{\prime}\right) \wedge^{\prime} \llbracket \varphi\left(u^{\prime}\right) \rrbracket^{H} \quad \text { (pois } \varepsilon \text { é sobrejetora) } \\
& =\llbracket \exists u^{\prime} \in x^{\prime} \varphi\left(u^{\prime}\right) \rrbracket^{H^{\prime}} \\
& \text { (por definição) } \\
& \text { (pois } f \text { preserva } \wedge, \bigvee \text { ) } \\
& \text { (por definição) }
\end{aligned}
$$


Similarmente, temos:

$$
\begin{array}{rlrl}
f\left(\llbracket \forall u \in x \varphi(u) \rrbracket^{H}\right) & =f\left(\bigwedge_{u \in \operatorname{dom}(x)} x(u) \rightarrow \llbracket \varphi(u) \rrbracket^{H}\right) & & \text { (por definição) } \\
& \leq \bigwedge_{u \in \operatorname{dom}(x)}^{\prime} f\left(x(u) \rightarrow \llbracket \varphi(u) \rrbracket^{H}\right) & \text { (pois } f \text { é crescente) } \\
& \leq \bigwedge_{u \in \operatorname{dom}(x)}^{\prime} f(x(u)) \rightarrow^{\prime} f\left(\llbracket \varphi(u) \rrbracket^{H}\right) & (\text { pois } f(a \rightarrow b) \leq f(a) \rightarrow f(b)) \\
& \leq \bigwedge_{u \in \operatorname{dom}(x)}^{\prime} f(x(u)) \rightarrow^{\prime} \llbracket \varphi(\varepsilon(u)) \rrbracket^{H^{\prime}} & \text { (por hipótese de indução) } \\
& =\bigwedge_{u \in \operatorname{dom}(x)}^{\prime} x^{\prime}(\varepsilon(u)) \rightarrow^{\prime} \llbracket \varphi(\varepsilon(u)) \rrbracket^{H^{\prime}} & & \text { (usando que } \left.\left(x, x^{\prime}\right) \in \tilde{f}\right) \\
& =\bigwedge_{u^{\prime} \in \operatorname{dom}\left(x^{\prime}\right)}^{\prime} x^{\prime}\left(u^{\prime}\right) \rightarrow^{\prime} \llbracket \varphi\left(u^{\prime}\right) \rrbracket^{H^{\prime}} & \text { (pois } \varepsilon \text { é sobrejetora) } \\
& =\llbracket \forall u^{\prime} \in x^{\prime} \varphi\left(u^{\prime}\right) \rrbracket^{H^{\prime}} & \text { (por definição) }
\end{array}
$$

Outra consequência do teorema é que, se $\llbracket x=z \rrbracket^{H}=1_{H}$, então, como $1_{H}=\bigwedge \emptyset$, obtemos:

$$
f\left(\llbracket x=z \rrbracket^{H}\right)=f\left(1_{H}\right)=1_{H^{\prime}} \leq \llbracket x^{\prime}=z^{\prime} \rrbracket^{H^{\prime}}
$$

ou seja, $\llbracket x^{\prime}=z^{\prime} \rrbracket^{H^{\prime}}=1_{H^{\prime}}$. Assim, ao passarmos ao quociente pela relação $\equiv$, a semifunção $\tilde{f}$ define uma função $\bar{f}: \mathbf{S e t}_{0}^{(H)} \rightarrow \mathbf{S e t}_{0}^{\left(H^{\prime}\right)}$.

Proposição 5.5.6. 1. $\overline{i d_{H}}=i d_{\operatorname{Set}_{0}^{(H)}}: \operatorname{Set}_{0}^{(H)} \rightarrow \operatorname{Set}_{0}^{(H)}$;

2. se $f^{\prime}: H^{\prime} \rightarrow H^{\prime \prime}$ preserva supremos arbitrários e infimos finitos, então $\overline{f^{\prime}} \circ \bar{f}=\overline{f^{\prime} \circ f}$ : $\operatorname{Set}_{0}^{(H)} \rightarrow$ Set $_{0}^{\left(H^{\prime \prime}\right)}$

Demonstração. $\quad$ 1. mostremos que, para todo $\alpha \in \operatorname{Ord}$, se $\left(x, y^{\prime}\right) \in\left(\widetilde{i d_{H}}\right)_{\alpha}$, então $\llbracket x=y^{\prime} \rrbracket^{H}=$ 1. Suponha, por indução, que isso ocorra para todo $\beta \in \operatorname{Ord} \operatorname{com} \beta<\alpha$, e seja $\varepsilon$ : $\operatorname{dom}(x) \rightarrow \operatorname{dom}\left(x^{\prime}\right)$, com $\llbracket x^{\prime}=y^{\prime} \rrbracket^{H}=1$, testemunha de $\left(x, y^{\prime}\right) \in\left(\widetilde{i d_{H}}\right)_{\alpha}$. Então, $x^{\prime} \circ \varepsilon=$ $i d_{H} \circ x=x$, e para todo $u \in \operatorname{dom}(x),(u, \varepsilon(x)) \in\left(\widetilde{i d_{H}}\right)_{\varrho(x)}$, donde, por hipótese de indução, $\llbracket \varepsilon(u)=u \rrbracket^{H}=1$. Com isso, para todo $u \in \operatorname{dom}(x)$, usando que $\varepsilon$ é sobrejetora obtemos:

$$
x(u)=x^{\prime}(\varepsilon(u))=x^{\prime}(\varepsilon(u)) \wedge \llbracket \varepsilon(u)=u \rrbracket^{H} \leq \bigvee_{w^{\prime} \in \operatorname{dom}\left(x^{\prime}\right)} x^{\prime}\left(w^{\prime}\right) \wedge \llbracket w^{\prime}=u \rrbracket^{H}=\llbracket u \in x^{\prime} \rrbracket^{H}
$$


Similarmente, para todo $v^{\prime} \in \operatorname{dom}\left(x^{\prime}\right)$, existe $v \in \operatorname{dom}(x)$ tal que $\varepsilon(v)=v^{\prime}$, e vale:

$$
x^{\prime}\left(v^{\prime}\right)=x^{\prime}(\varepsilon(v))=x(v)=x(v) \wedge \llbracket \varepsilon(v)=v \rrbracket^{H} \leq \bigvee_{w \in \operatorname{dom}(x)} x(w) \wedge \llbracket w=v^{\prime} \rrbracket^{H}=\llbracket v^{\prime} \in x \rrbracket^{H}
$$

Agora, observe que $x(u) \leq \llbracket u \in x^{\prime} \rrbracket^{H}$ se, e somente se, $1 \leq x(u) \rightarrow \llbracket u \in x^{\prime} \rrbracket^{H}$, para todo $u \in \operatorname{dom}(x)$; ou seja, $\bigwedge_{u \in \operatorname{dom}(x)} x(u) \rightarrow \llbracket u \in x^{\prime} \rrbracket^{H}=1$. De forma análoga, $x^{\prime}\left(v^{\prime}\right) \leq \llbracket v^{\prime} \in x^{\prime} \rrbracket^{H}$ para todo $v^{\prime} \in \operatorname{dom}\left(x^{\prime}\right)$ equivale a $\bigwedge_{v^{\prime} \in \operatorname{dom}\left(x^{\prime}\right)} x^{\prime}\left(v^{\prime}\right) \rightarrow \llbracket v^{\prime} \in x \rrbracket^{H}=1$. Portanto:

$$
\llbracket x=x^{\prime} \rrbracket=\bigwedge_{u \in \operatorname{dom}(x)}\left(x(u) \rightarrow \llbracket u \in x^{\prime} \rrbracket^{H}\right) \wedge \bigwedge_{v^{\prime} \in \operatorname{dom}\left(x^{\prime}\right)}\left(x^{\prime}\left(v^{\prime}\right) \rightarrow \llbracket v^{\prime} \in x \rrbracket^{H}\right)=1
$$

Finalmente, como temos que $\llbracket x=x^{\prime} \rrbracket^{H}=1=\llbracket x^{\prime}=y^{\prime} \rrbracket^{H}$, concluímos que $\llbracket x=y^{\prime} \rrbracket^{H}=1$, como queríamos.

Agora, pela definição da relação de equivalência, temos que $\llbracket x=y^{\prime} \rrbracket^{H}=1$ se, e somente se, $[x]=\left[y^{\prime}\right]$. Logo, passando ao quociente, $\left([x],\left[y^{\prime}\right]\right) \in \overline{i d_{H}}$ se, e somente se, $[x]=\left[y^{\prime}\right]$, e $\overline{i d_{H}}$ é a identidade de $\mathbf{S e t}_{0}^{(H)}$.

2. Seja $\left([x],\left[z^{\prime \prime}\right]\right) \in \overline{f^{\prime}} \circ \bar{f}$, isto é, existe $\left[y^{\prime}\right] \in \operatorname{Set}_{0}^{(H)}$ tal que $\left([x],\left[y^{\prime}\right]\right) \in \bar{f}$ e $\left(\left[y^{\prime}\right],\left[z^{\prime \prime}\right]\right) \in \overline{f^{\prime}}$. Considere $\varepsilon: \operatorname{dom}(x) \rightarrow \operatorname{dom}\left(x^{\prime}\right), \operatorname{com} x^{\prime} \in\left[y^{\prime}\right]$, uma testemunha de $\left([x],\left[y^{\prime}\right]\right) \in \bar{f}$, e $\varepsilon^{\prime}: \operatorname{dom}\left(x^{\prime}\right) \rightarrow \operatorname{dom}\left(y^{\prime \prime}\right), \operatorname{com} y^{\prime \prime} \in\left[z^{\prime \prime}\right]$, uma testemunha de $\left(\left[y^{\prime}\right],\left[z^{\prime \prime}\right]\right)=\left(\left[x^{\prime}\right],\left[z^{\prime \prime}\right]\right) \in \overline{f^{\prime}}$. Então, $\varepsilon^{\prime} \circ \varepsilon: \operatorname{dom}(x) \rightarrow \operatorname{dom}\left(y^{\prime \prime}\right)$ é uma testemunha de $\left([x],\left[z^{\prime \prime}\right]\right) \in \overline{f^{\prime} \circ f}$. Ou seja, mostramos que $\overline{f^{\prime}} \circ \bar{f} \subseteq \overline{f^{\prime} \circ f}$, e como ambas são funções, concluímos que $\overline{f^{\prime}} \circ \bar{f}=\overline{f^{\prime} \circ f}$.

Finalmente, usando que $f\left(\llbracket y \in x \rrbracket^{H}\right) \leq^{\prime} \llbracket y^{\prime} \in x^{\prime} \rrbracket^{H^{\prime}}$, não é difícil mostrar que, se $\llbracket h: x \rightarrow$ $y$ é função $\rrbracket^{H}=1_{H}$, então $\llbracket \tilde{f}(h): \tilde{f}(x) \rightarrow \tilde{f}(y)$ é função $\rrbracket^{H^{\prime}}=1_{H^{\prime}}$. Ademais, vale:

$$
\tilde{f}\left(i d_{x}\right)=i d_{\tilde{f}(x)}: \tilde{f}(x) \rightarrow \tilde{f}(x) \quad \text { e } \quad \llbracket \tilde{f}\left(i d_{x}\right): \tilde{f}(x) \rightarrow \tilde{f}(x) \text { é função } \rrbracket^{H^{\prime}}=1_{H^{\prime}}
$$

e, se $\llbracket g: y \rightarrow z$ é função $\rrbracket^{H}=1_{H}$, então:

$$
\tilde{f}(g \circ h)=\tilde{f}(g) \circ \tilde{f}(h): \tilde{f}(x) \rightarrow \tilde{f}(z) \quad \text { e } \quad \llbracket \tilde{f}(g \circ h): \tilde{f}(x) \rightarrow \tilde{f}(z) \text { é função } \rrbracket^{H^{\prime}}=1_{H^{\prime}}
$$

Isto é, ao passarmos ao quociente, $\bar{f}: \mathbf{S e t}^{(H)} \rightarrow \operatorname{Set}^{\left(H^{\prime}\right)}$ define um funtor.

Já vimos em seções anteriores que uma função entre álgebras de Heyting que preserva 
supremos arbitrários e ínfimos finitos induz um funtor entre os topoi de feixes correspondentes que preserva colimites arbitrários e limites finitos (parte esquerda de um morfismo geométrico). Mais precisamente, usando as equivalências naturais $H$-Set $\simeq \mathbf{S h}(H)$ e $H^{\prime}$-Set $\simeq \mathbf{S h}\left(H^{\prime}\right)$, a função $f: H \rightarrow H^{\prime}$ dá origem a uma função $\varphi_{f}: H$-Set $\rightarrow H^{\prime}$-Set dada por:

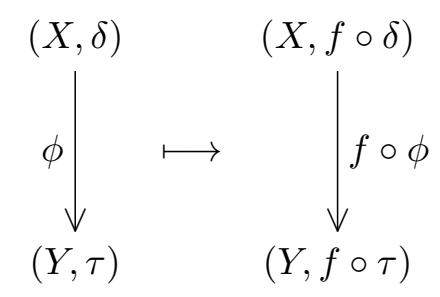

onde $f \circ \phi: X \times Y \rightarrow H^{\prime}$. Com isso, vejamos como $\tilde{f}$ pode induzir um morfismo de $H^{\prime}$-sets.

Proposição 5.5.7. Sejam $\left(x, x^{\prime}\right) \in \tilde{f} e \varepsilon: \operatorname{dom}(x) \rightarrow \operatorname{dom}\left(x^{\prime}\right)$ testemunhando isso. Considere a função $\varepsilon^{H^{\prime}}: \operatorname{dom}(x) \times \operatorname{dom}\left(x^{\prime}\right) \rightarrow H^{\prime}$ dada por:

$\varepsilon^{H^{\prime}}\left(u, v^{\prime}\right):=f\left(\llbracket u \in x \rrbracket^{H}\right) \wedge^{\prime} \llbracket \varepsilon(u)=v^{\prime} \rrbracket^{H^{\prime}} \wedge^{\prime} \llbracket v^{\prime} \in x^{\prime} \rrbracket^{H^{\prime}}$, para todo $\left(u, v^{\prime}\right) \in \operatorname{dom}(x) \times \operatorname{dom}\left(x^{\prime}\right)$

Então, $\varepsilon^{H^{\prime}}$ define um morfismo de $H$-sets $\varepsilon^{H^{\prime}}:\left(\operatorname{dom}(x), f \circ \delta_{x}\right) \rightarrow\left(\operatorname{dom}\left(x^{\prime}\right), \delta_{x^{\prime}}\right)$ que não depende da escolha da testemunha, onde,

$$
\begin{gathered}
\delta_{x}(u, v):=\llbracket u \in x \rrbracket^{H} \wedge \llbracket u=v \rrbracket^{H}, \text { para todos } u, v \in \operatorname{dom}(x) \\
\delta_{x^{\prime}}\left(u^{\prime}, v^{\prime}\right):=\llbracket u^{\prime} \in x^{\prime} \rrbracket^{H^{\prime}} \wedge^{\prime} \llbracket u^{\prime}=v^{\prime} \rrbracket^{H^{\prime}}, \text { para todos } u^{\prime}, v^{\prime} \in \operatorname{dom}\left(x^{\prime}\right)
\end{gathered}
$$

Note que $\delta_{x}$ e $\delta_{x^{\prime}}$ são exatamente as mesmas usadas na equivalência entre $\mathbf{S e t}^{(H)}$ e $H$-Set, pois, como $\llbracket u \in x \rrbracket^{H} \wedge \llbracket u=v \rrbracket^{H} \leq \llbracket v \in x \rrbracket$, temos:

$$
\llbracket u \in x \rrbracket^{H} \wedge \llbracket u=v \rrbracket^{H} \wedge \llbracket v \in x \rrbracket^{H}=\llbracket u \in x \rrbracket^{H} \wedge \llbracket u=v \rrbracket^{H}
$$

Demonstração. Verifiquemos as quatro condições de morfismo de $H$-sets. Sejam $u, v \in \operatorname{dom}(x)$ e $u^{\prime}, v^{\prime} \in \operatorname{dom}\left(x^{\prime}\right)$.

1. $\delta_{x^{\prime}}\left(u^{\prime}, v^{\prime}\right) \wedge^{\prime} \varepsilon^{H^{\prime}}\left(u, v^{\prime}\right) \leq \varepsilon^{H^{\prime}}\left(u, u^{\prime}\right)$. De fato,

$$
\begin{aligned}
& \delta_{x^{\prime}}\left(u^{\prime}, v^{\prime}\right) \wedge^{\prime} \varepsilon^{H^{\prime}}\left(u, v^{\prime}\right)= \\
& \quad=\llbracket u^{\prime} \in x^{\prime} \rrbracket^{H^{\prime}} \wedge^{\prime} \llbracket u^{\prime}=v^{\prime} \rrbracket^{H^{\prime}} \wedge^{\prime} f\left(\llbracket u \in x \rrbracket^{H}\right) \wedge^{\prime} \llbracket \varepsilon(u)=v^{\prime} \rrbracket^{H^{\prime}} \wedge^{\prime} \llbracket v^{\prime} \in x^{\prime} \rrbracket^{H^{\prime}} \\
& \quad \leq f\left(\llbracket u \in x \rrbracket^{H}\right) \wedge^{\prime} \llbracket \varepsilon(u)=u^{\prime} \rrbracket^{H^{\prime}} \wedge^{\prime} \llbracket u^{\prime} \in x^{\prime} \rrbracket^{H^{\prime}}
\end{aligned}
$$




$$
=\varepsilon^{H^{\prime}}\left(u, u^{\prime}\right)
$$

2. $\left(f \circ \delta_{x}\right)(u, v) \wedge^{\prime} \varepsilon^{H^{\prime}}\left(u, v^{\prime}\right) \leq \varepsilon^{H^{\prime}}\left(v, v^{\prime}\right)$. De fato,

$$
\begin{aligned}
& \left(f \circ \delta_{x}\right)(u, v) \wedge^{\prime} \varepsilon^{H^{\prime}}\left(u, v^{\prime}\right)= \\
& \quad=f\left(\llbracket u \in x \rrbracket^{H} \wedge \llbracket u=v \rrbracket^{H}\right) \wedge^{\prime} f\left(\llbracket u \in x \rrbracket^{H}\right) \wedge^{\prime} \llbracket \varepsilon(u)=v^{\prime} \rrbracket^{H^{\prime}} \wedge^{\prime} \llbracket v^{\prime} \in x^{\prime} \rrbracket^{H^{\prime}} \\
& \quad \leq f\left(\llbracket v \in x \rrbracket^{H}\right) \wedge^{\prime} \llbracket \varepsilon(u)=\varepsilon(v) \rrbracket^{H^{\prime}} \wedge^{\prime} \llbracket \varepsilon(u)=v^{\prime} \rrbracket^{H^{\prime}} \wedge^{\prime} \llbracket v^{\prime} \in x^{\prime} \rrbracket^{H^{\prime}} \\
& \quad \leq f\left(\llbracket v \in x \rrbracket^{H}\right) \wedge^{\prime} \llbracket \varepsilon(v) \in v^{\prime} \rrbracket^{H^{\prime}} \wedge^{\prime} \llbracket v^{\prime} \in x^{\prime} \rrbracket^{H^{\prime}} \\
& \quad=\varepsilon^{H^{\prime}}\left(v, v^{\prime}\right)
\end{aligned}
$$

3. $\varepsilon^{H^{\prime}}\left(u, u^{\prime}\right) \wedge \varepsilon^{H^{\prime}}\left(u, v^{\prime}\right) \leq \delta_{x^{\prime}}\left(u^{\prime}, v^{\prime}\right)$. De fato,

$$
\begin{aligned}
& \varepsilon^{H^{\prime}}\left(u, u^{\prime}\right) \wedge \varepsilon^{H^{\prime}}\left(u, v^{\prime}\right)= \\
& \quad=f\left(\llbracket u \in x \rrbracket^{H}\right) \wedge^{\prime} \llbracket \varepsilon(u)=u^{\prime} \rrbracket^{H^{\prime}} \wedge^{\prime} \llbracket u^{\prime} \in x^{\prime} \rrbracket^{H^{\prime}} \wedge^{\prime} f\left(\llbracket u \in x \rrbracket^{H}\right) \wedge^{\prime} \\
& \wedge^{\prime} \llbracket \varepsilon(u)=v^{\prime} \rrbracket^{H^{\prime}} \wedge^{\prime} \llbracket v^{\prime} \in x^{\prime} \rrbracket^{H^{\prime}} \\
& \quad \leq f\left(\llbracket u \in x \rrbracket^{H}\right) \wedge^{\prime} \llbracket u^{\prime}=v^{\prime} \rrbracket^{H^{\prime}} \wedge^{\prime} \llbracket u^{\prime} \in x^{\prime} \rrbracket^{H^{\prime}} \wedge^{\prime} \llbracket v^{\prime} \in x^{\prime} \rrbracket^{H^{\prime}} \\
& \quad \leq \llbracket v^{\prime} \in x^{\prime} \rrbracket^{H^{\prime}} \wedge^{\prime} \llbracket u^{\prime}=v^{\prime} \rrbracket^{H^{\prime}} \\
& \quad=\delta_{x^{\prime}}\left(u^{\prime}, v^{\prime}\right)
\end{aligned}
$$

4. $\bigvee_{w^{\prime} \in \operatorname{dom}\left(x^{\prime}\right)}^{\prime} \varepsilon^{H^{\prime}}\left(u, w^{\prime}\right)=\left(f \circ \delta_{x}\right)(u, u)$. De fato,

- para todo $w^{\prime} \in \operatorname{dom}\left(x^{\prime}\right)$, usando que $f$ preserva $1_{H}$, temos:

$$
\begin{aligned}
\varepsilon^{H^{\prime}}\left(u, w^{\prime}\right) & =f\left(\llbracket u \in x \rrbracket^{H}\right) \wedge^{\prime} \llbracket \varepsilon(u)=w^{\prime} \rrbracket^{H^{\prime}} \wedge^{\prime} \llbracket w^{\prime} \in x^{\prime} \rrbracket^{H^{\prime}} \\
& \leq f\left(\llbracket u \in x \rrbracket^{H}\right) \wedge^{\prime} 1_{H^{\prime}}=f\left(\llbracket u \in x \rrbracket^{H}\right) \wedge^{\prime} f\left(1_{H}\right) \\
& =f\left(\llbracket u \in x \rrbracket^{H}\right) \wedge^{\prime} f\left(\llbracket u \in u \rrbracket^{H}\right)=f\left(\llbracket u \in x \rrbracket^{H} \wedge \llbracket u=u \rrbracket^{H}\right) \\
& =\left(f \circ \delta_{x}\right)(u, u)
\end{aligned}
$$

Logo, $\bigvee_{w^{\prime} \in \operatorname{dom}\left(x^{\prime}\right)}^{\prime} \varepsilon^{H^{\prime}}\left(u, w^{\prime}\right) \leq\left(f \circ \delta_{x}\right)(u, u) ;$

- por outro lado, como $\varepsilon(u) \in \operatorname{dom}\left(x^{\prime}\right)$, temos:

$$
\begin{aligned}
\bigvee_{w^{\prime} \in \operatorname{dom}\left(x^{\prime}\right)}^{\prime} \varepsilon^{H^{\prime}}\left(u, w^{\prime}\right) & \geq \varepsilon^{H^{\prime}}(u, \varepsilon(u))= \\
& =f\left(\llbracket u \in x \rrbracket^{H}\right) \wedge^{\prime} \llbracket \varepsilon(u)=\varepsilon(u) \rrbracket^{H^{\prime}} \wedge^{\prime} \llbracket \varepsilon(u) \in x^{\prime} \rrbracket^{H^{\prime}}
\end{aligned}
$$




$$
\begin{aligned}
& =f\left(\llbracket u \in x \rrbracket^{H}\right) \wedge^{\prime} 1_{H^{\prime}} \wedge^{\prime} \llbracket \varepsilon(u) \in x^{\prime} \rrbracket^{H^{\prime}} \\
& =f\left(\llbracket u \in x \rrbracket^{H}\right) \wedge^{\prime} \llbracket \varepsilon(u) \in x^{\prime} \rrbracket^{H^{\prime}} \\
& \geq f\left(\llbracket u \in x \rrbracket^{H}\right) \wedge^{\prime} f\left(\llbracket u \in x \rrbracket^{H}\right)=f\left(\llbracket u \in x \rrbracket^{H}\right) \\
& =f\left(\llbracket u \in x \rrbracket^{H} \wedge 1_{H}\right)=f\left(\llbracket u \in x \rrbracket^{H} \wedge \llbracket u=u \rrbracket^{H}\right) \\
& =\left(f \circ \delta_{x}\right)(u, u)
\end{aligned}
$$

$$
\text { Logo, } \underset{w^{\prime} \in \operatorname{dom}\left(x^{\prime}\right)}{\bigvee^{\prime}} \varepsilon^{H^{\prime}}\left(u, w^{\prime}\right) \geq\left(f \circ \delta_{x}\right)(u, u) \text {. }
$$

Enfim, observe que o resultado não depende da escolha da testemunha: sejam $\left(u, v^{\prime}\right) \in \operatorname{dom}(x) \times$ $\operatorname{dom}\left(x^{\prime}\right)$ e $\tau: \operatorname{dom}(x) \rightarrow \operatorname{dom}\left(x^{\prime}\right)$ que testemunha $\left(x, x^{\prime}\right) \in \tilde{f}$. Então, como $u \in \operatorname{dom}(x)$, temos $(u, \varepsilon(u)) \in \tilde{f}$ e $(u, \tau(u)) \in \tilde{f}$; e como $1_{H}=\llbracket u=u \rrbracket^{H}$, pelo teorema anterior

$$
1_{H^{\prime}}=f\left(1_{H}\right)=f\left(\llbracket u=u \rrbracket^{H}\right) \leq \llbracket \tau(u)=\varepsilon(u) \rrbracket^{H^{\prime}}
$$

Dessa forma,

$$
\begin{aligned}
\varepsilon^{H^{\prime}}\left(u, v^{\prime}\right) & =f\left(\llbracket u \in x \rrbracket^{H}\right) \wedge^{\prime} \llbracket \varepsilon(u)=v^{\prime} \rrbracket^{H^{\prime}} \wedge^{\prime} \llbracket v^{\prime} \in x^{\prime} \rrbracket^{H^{\prime}}= \\
& =f\left(\llbracket u \in x \rrbracket^{H}\right) \wedge^{\prime} 1_{H^{\prime}} \wedge^{\prime} \llbracket \varepsilon(u)=v^{\prime} \rrbracket^{H^{\prime}} \wedge^{\prime} \llbracket v^{\prime} \in x^{\prime} \rrbracket^{H^{\prime}} \\
& =f\left(\llbracket u \in x \rrbracket^{H}\right) \wedge^{\prime} \llbracket \tau(u)=\varepsilon(u) \rrbracket^{H^{\prime}} \wedge^{\prime} \llbracket \varepsilon(u)=v^{\prime} \rrbracket^{H^{\prime}} \wedge^{\prime} \llbracket v^{\prime} \in x^{\prime} \rrbracket^{H^{\prime}} \\
& \leq f\left(\llbracket u \in x \rrbracket^{H}\right) \wedge^{\prime} \llbracket \tau(u)=v^{\prime} \rrbracket^{H^{\prime}} \wedge^{\prime} \llbracket v^{\prime} \in x^{\prime} \rrbracket^{H^{\prime}} \\
& =\tau^{H^{\prime}}\left(u, v^{\prime}\right)
\end{aligned}
$$

donde $\varepsilon^{H^{\prime}}\left(u, v^{\prime}\right) \leq \tau^{H^{\prime}}\left(u, v^{\prime}\right)$. A verificação que $\tau^{H^{\prime}}\left(u, v^{\prime}\right) \leq \varepsilon^{H^{\prime}}\left(u, v^{\prime}\right)$ é análoga.

Observação. A ideia agora seria mostrar que tais morfismos $\varepsilon^{H^{\prime}}$ são isos, e poderiam ser usados para construir um isomorfismo natural entre $\bar{f}$ e $\varphi_{f}$. Para tanto, uma possibilidade seria usar a caracterização de monos e epis em $H$-Set (ver proposições 2.8.8 e 2.8.7 de [Bor08c]), ou seja, para todos $u, v \in \operatorname{dom}(x)$ e $u^{\prime} \in \operatorname{dom}\left(x^{\prime}\right)$ mostrar que:

- $\varepsilon^{H^{\prime}}\left(u, u^{\prime}\right) \wedge \varepsilon^{H^{\prime}}\left(v, u^{\prime}\right) \leq\left(f \circ \delta_{x}\right)(u, v)$ (o que equivale a $\varepsilon^{H^{\prime}}$ ser mono);

- $\bigvee_{w \in \operatorname{dom}(x)} \varepsilon^{H^{\prime}}\left(w, u^{\prime}\right)=\delta_{x^{\prime}}\left(u^{\prime}, u^{\prime}\right)$ (o que equivale a $\varepsilon^{H^{\prime}}$ ser epi);

e, como $H$-Set é um topos, $\varepsilon^{H^{\prime}}$ seria um iso. 
Agora, abrindo as definições, obtemos:

$$
\begin{aligned}
& \varepsilon^{H^{\prime}}\left(u, u^{\prime}\right) \wedge \varepsilon^{H^{\prime}}\left(v, u^{\prime}\right)= \\
& =f\left(\llbracket u \in x \rrbracket^{H}\right) \wedge^{\prime} \llbracket \varepsilon(u)=u^{\prime} \rrbracket^{H^{\prime}} \wedge^{\prime} \llbracket u^{\prime} \in x^{\prime} \rrbracket^{H^{\prime}} \wedge^{\prime} f\left(\llbracket v \in x \rrbracket^{H}\right) \wedge^{\prime} \llbracket \varepsilon(v)=u^{\prime} \rrbracket^{H^{\prime}} \wedge^{\prime} \llbracket u^{\prime} \in x^{\prime} \rrbracket^{H^{\prime}} \\
& \leq f\left(\llbracket u \in x \rrbracket^{H}\right) \wedge^{\prime} \llbracket \varepsilon(u)=u^{\prime} \rrbracket^{H^{\prime}} \wedge^{\prime} \llbracket \varepsilon(v)=u^{\prime} \rrbracket^{H^{\prime}} \\
& \leq f\left(\llbracket u \in x \rrbracket^{H}\right) \wedge^{\prime} \llbracket \varepsilon(u)=\varepsilon(v) \rrbracket^{H^{\prime}} \\
& \text { e queremos mostrar que } \leq f\left(\llbracket u \in x \rrbracket^{H} \wedge \llbracket u=v \rrbracket^{H}\right)=\left(f \circ \delta_{x}\right)(u . v) \\
& \bigvee_{w \in \operatorname{dom}(x)} \varepsilon^{H^{\prime}}\left(w, u^{\prime}\right)=\bigvee_{w \in \operatorname{dom}(x)} f\left(\llbracket w \in x \rrbracket^{H}\right) \wedge^{\prime} \llbracket \varepsilon(w)=u^{\prime} \rrbracket^{H^{\prime}} \wedge^{\prime} \llbracket u^{\prime} \in x^{\prime} \rrbracket^{H^{\prime}} \\
& =\bigvee_{w \in \operatorname{dom}(x)} f\left(\llbracket w \in x \rrbracket^{H}\right) \wedge^{\prime} \llbracket \varepsilon(w)=\varepsilon(t) \rrbracket^{H^{\prime}} \wedge^{\prime} \llbracket \varepsilon(t) \in x^{\prime} \rrbracket^{H^{\prime}} \text { (pois } \varepsilon \text { é sobre) } \\
& \geq f\left(\llbracket t \in x \rrbracket^{H}\right) \wedge^{\prime} \llbracket \varepsilon(t)=\varepsilon(t) \rrbracket^{H^{\prime}} \wedge^{\prime} \llbracket \varepsilon(t) \in x^{\prime} \rrbracket^{H^{\prime}} \\
& \text { e queremos mostrar que }=\llbracket u^{\prime} \in x^{\prime} \rrbracket^{H^{\prime}}=\delta_{x^{\prime}}\left(u^{\prime}, u^{\prime}\right)
\end{aligned}
$$

(a outra desigualdade para a condição de epi é imediata, por conta do ínfimo). Não parece claro que, com o que fizemos até aqui, consigamos obter as desigualdades desejadas acima, pois ambas dependem não apenas a preservação da $H$-valoração das fórmulas atômicas por $f$ do teorema 5.5.4, mas da preservação estrita, isto é: se $\left(x, x^{\prime}\right),\left(y, y^{\prime}\right),\left(z, z^{\prime}\right) \in \tilde{f}$, então

$$
f\left(\llbracket x \in y \rrbracket^{H}\right)=\llbracket x^{\prime} \in y^{\prime} \rrbracket^{H^{\prime}} \quad f\left(\llbracket x=z \rrbracket^{H}\right)=\llbracket x^{\prime}=z^{\prime \prime} \rrbracket^{H^{\prime}}
$$

Assim, observando a demonstração do teorema 5.5.4, note que podemos obter essas igualdades (e, logo, que $\varepsilon^{H^{\prime}}$ é iso) pelo menos no caso em que $f: H \rightarrow H^{\prime}$ preserva (estritamente) a implicação, e tanto supremos quanto ínfimos arbitrários. Com essa hipótese sobre $f$, também poderíamos adaptar o corolário do teorema para obter a preservação estrita da $H$-valoração de todas as fórmulas com quantificadores restritos.

\subsection{Considerações finais}

No segundo semestre de 2018, dedicamo-nos a descrever aplicações possivelmente novas de lógica de topos em análise funcional, motivados pelos trabalhos de Mulvey e Banachewski ([Mul80], [BM06]); por exemplo, analisar se para uma definição razoável de espaços de Hilbert 
num topos valeria o resultado de reflexividade.

Em [Mul80], são dadas três descrições de feixes de Banach: uma definindo espaços de Banach num topos espacial usando lógica interna, outra usando uma variante de feixe geométrico, e uma última usando uma variante de feixe funtorial. Infelizmente, não localizamos na literatura uma forma razoável de definir espaços de Hilbert num topos, nem como realizar construções adequadas de (variantes de) feixe geométrico e feixe funtorial para o caso Hilbert.

Por outro lado, também tentamos, sem sucesso, chegar a um conceito unificador de estruturas métricas completas num topos que cobrisse os dois casos já feitos (espaços de Banach e $C^{\star}$-álgebras) e um eventual caso Hilbert. Entretanto, consideramos que uma possível lógica contínua num topos seja um bom candidato à investigação futura, em uma linha semelhante à explorada em [AH16].

Já no primeiro semestre de 2019, motivados pela conexão "folclórica" (no sentido de ser frequentemente mencionada, porém não feita de forma explícita) entre forcing sobre um conjunto parcialmente ordenado $(P, \leq)$ e a semântica de Kripke-Joyal em $\mathbf{S h}(\mathcal{O}(P))$, fomos na direção de tentar explicitar a naturalidade (em termos de coerência entre morfismos) dessa correspondência. Os resultados iniciais dessa investigação são o conteúdo da seção anterior: como relacionar morfismos geométricos $\mathbf{S h}(H) \rightarrow \mathbf{S h}\left(H^{\prime}\right)$ com morfismos em $V^{(H)} \rightarrow V^{\left(H^{\prime}\right)}$ obtidos recursivamente de morfismos geométricos $H \rightarrow H^{\prime}$.

Com respeito à correspondência semântica, se considerarmos uma relação de forcing sobre $(P, \leq)$, os elementos $p \in P$ determinam uma família geradora $\left\{H\left(-, \mathcal{O}_{p}\right) \mid p \in P\right\}$ em $\mathbf{S h}(H)$ (para $H=\mathcal{O}(P)$ ). Logo, para essa família podemos considerar a semântica de Kripke-Joyal em $\mathbf{S h}(H) \simeq H$-Set $\simeq \mathbf{S e t}^{(H)}$.

Contudo, não está claro que a relação entre forcing e Kripke-Joyal indicada no final da seção VI.7 de [LM94] pode ser verificada explicitamente levando-se em conta os funtores de equivalência $H$-Set $\simeq \mathbf{S e t}^{(H)}$ apresentado acima ou a equivalência $\mathbf{S h}(H) \simeq \mathbf{S e t}^{(H)}$ feita em [BS89] (lembrando que $p \Perp \vdash \varphi(\bar{a})$ depende apenas de $[\bar{a}]$ em Set $\left.^{(H)}\right)$. A dificuldade central é no nível das sentenças atômicas, pois na semântica de Kripke-Joyal a relação é "on spot" (como na verdade de Tarski) enquanto em $V^{(H)}$ ela é recursiva.

Também gera estranhamento o fato que, apesar da lógica interna do topos ser (em geral) intuicionista, a semântica de Kripke-Joyal se assemelha mais às propriedades do forcing clássico do que ao intuicionista. Uma possibilidade de caminho para esclarecer essa questão é investigar 
uma forma mais geral da semântica de Kripke-Joyal: a semântica stack, como apresentada em [Shu10]. Continuaremos trabalhando sobre o tema em [ACM19].

Outro ponto interessante nessa direção é estudar se a relação entre forcing clássico e forcing intuicionista está relacionada com as funções $\operatorname{Reg}(H) \hookrightarrow H$ e $H \rightarrow \frac{H}{\langle x \leftrightarrow \neg \neg x\rangle}$, e as semânticas de Kripke-Joyal correspondentes.

Indo numa direção diferente, outro aspecto a ser analisado é o dos funtores lógicos. Lembrando da proposição 3.4.9 que funtores lógicos coincidem com morfismos geométricos apenas no caso de equivalências de categorias, essa "ortogonalidade" entre os conceitos já pode ser observada em nível algébrico. Dada uma álgebra de Boole completa não trivial $(B, \leq)$ e um morfismo injetor $2 \hookrightarrow B(\operatorname{com} \mathbf{2}=\{0,1\})$, obtemos três tipos de morfismo $B \rightarrow \mathbf{2}$ :

- $l: B \rightarrow \mathbf{2}$ adjunto à esquerda, dado por $l(x)=0 \Leftrightarrow x=0$, que só preserva supremos;

- $r: B \rightarrow \mathbf{2}$ adjunto à direita, dado por $r(x)=1 \Leftrightarrow x=1$, que só preserva ínfimos;

- $U: B \rightarrow \mathbf{2}$ o quociente por ultrafiltros, que preserva 0,1 , negação, implicação, e supremos e ínfimos finitos.

Por outro lado, observe que um funtor lógico $\mathbf{S h}(H) \rightarrow \mathbf{S h}\left(H^{\prime}\right)$ induz um morfismo de álgebras de Heyting $H \rightarrow H^{\prime}$ (pois $H \cong S u b(\mathbf{1})$ ). Parece natural, assim, tentar estabelecer a correspondência entre outros morfismos $H \rightarrow H^{\prime}$ e funtores lógicos, e investigar como se relacionam com morfismos induzidos em $V^{(H)} \rightarrow V^{\left(H^{\prime}\right)}$. 


\section{Apêndice A}

\section{Teoria dos reticulados}

O objetivo desse apêndice é servir de referência rápida para definições e resultados da teoria de reticulados (importantes no estudo de locales), e em especial de álgebras de Heyting e de Boole (usadas no estudo da lógica). Para uma introdução geral mais completa sobre reticulados pode-se consultar [Rut65], enquanto [Mir06] (nos capítulos 3 a 8) e [Bor08c] (seções 1.1 e 1.2) cobrem com maior riqueza de detalhes aspectos da teoria voltados para o estudo de feixes e sob um ponto de vista mais categorial.

\section{A.1 Reticulados}

Definição A.1.1. Um conjunto parcialmente ordenado $(P, \leq)$ é dito um reticulado se, para todos $a, b \in P$, existe o ínfimo (maior cota inferior) de $\{a, b\}$, denotado $a \wedge b$, e o supremo (menor cota superior) de $\{a, b\}$, denotado $a \vee b$.

Proposição A.1.2. Sejam $(P, \leq)$ um reticulado e $a, b, c \in P$. Então,

1. comutatividade: $a \wedge b=b \wedge a e a \vee b=b \vee a$;

2. associatividade: $a \wedge(b \wedge c)=(a \wedge b) \wedge c e a \vee(b \vee c)=(a \vee b) \vee c$;

3. $\operatorname{absorça\tilde {a}o:~} a \wedge(a \vee b)=a$ e $a \vee(a \wedge b)=a$.

A associatividade nos garante a existência do ínfimo e do supremo para qualquer subconjunto finito não vazio $S \subseteq P$, denotados, respectivamente, por $\bigwedge S$ e $\bigvee S$. 
Esses três pares de equações, aliás, são condição suficiente para $(P, \leq)$ ser um reticulado, logo fornecem uma definição equacional de reticulado (basta tomar uma das equivalências do item (2) abaixo como a definição da ordem).

Proposição A.1.3. Sejam $(P, \leq)$ um reticulado, $S, R \subseteq P$ subconjuntos finitos não vazios $e$ $a, b, c, d \in P$. Então,

1. $a \wedge a=a$ e $a \vee a=a$;

2. $a=a \wedge b \Leftrightarrow a \leq b \Leftrightarrow b=a \vee b$

3. $\bigvee(S \cup R)=(\bigvee S) \vee(\bigvee R)$ e $\bigwedge(S \cap R)=(\bigwedge S) \wedge(\bigwedge R)$;

4. se $S \subseteq R$, então $\bigvee S \leq \bigvee R$ e $\wedge R \leq \bigwedge S$;

5. se $a \leq b$ e $c \leq d$, então $a \wedge c \leq b \wedge d$ e $a \vee c \leq b \vee d$.

Definição A.1.4. Um reticulado $(P, \leq)$ é dito limitado se possui maior elemento (também chamado de unidade, denotado 1) e menor elemento (também chamado de zero, denotado 0 ).

Note que $1=\bigwedge \emptyset$ e $0=\bigvee \emptyset$; assim, equivalentemente, poderíamos definir um reticulado limitado exigindo que todo conjunto finito possua ínfimo e supremo.

Proposição A.1.5. Sejam $(P, \leq)$ um reticulado limitado e $a, b \in P$. Então:

1. $1 \wedge a=a$ e $0 \vee a=a$;

2. $0 \wedge a=0$ e $1 \vee a=1$;

3. se $a \vee b=0$, então $a=b=0$;

4. se $a \wedge b=1$, então $a=b=1$.

Definição A.1.6. Um reticulado $(P, \leq)$ é dito distributivo se satisfaz as leis distributivas ${ }^{1}$, isto é, para todos $a, b, c \in P$ :

$$
a \wedge(b \vee c)=(a \wedge b) \vee(a \wedge c) \quad \text { e } \quad a \vee(b \wedge c)=(a \vee b) \wedge(a \vee c)
$$

Proposição A.1.7. Sejam $(P, \leq)$ um reticulado distributivo e $S \subseteq P$ finito e não vazio. Então:

1. $a \vee(\bigwedge S)=\bigwedge\{a \vee s \mid s \in S\}$, para todo $a \in P$;

\footnotetext{
${ }^{1}$ Seria suficiente satisfazer uma delas, pois isso já implicaria na outra
} 
2. $a \wedge(\bigvee S)=\bigvee\{a \wedge s \mid s \in S\}$, para todo $a \in P$.

Definição A.1.8. Um reticulado $(P, \leq)$ é dito completo se todo subconjunto possui ínfimo e supremo.

\section{A.2 Álgebras de Heyting}

Definição A.2.1. Uma álgebra de Heyting é um reticulado $\operatorname{limitado}^{2}(H, \leq)$ tal que, para todos $a, b \in H$, existe o máximo

$$
(a \rightarrow b):=\operatorname{máx}\{c \in H \mid c \wedge a \leq b\}
$$

Assim, para todos $a, b, c \in H$, vale

$$
c \leq(a \rightarrow b) \quad \text { se, e somente se, } \quad a \wedge c \leq b
$$

Novamente, pode-se dar uma descrição equacional de uma álgebra de Heyting, como um reticulado limitado satisfazendo as identidades dos três primeiros itens da proposição a seguir.

Proposição A.2.2. Seja $(H, \leq)$ uma álgebra de Heyting. Então, para todos $a, b, c \in H$,

1. $a \wedge(a \rightarrow b)=a \wedge b$

2. $a \wedge(b \rightarrow c)=a \wedge((a \wedge b) \rightarrow(a \wedge c))$;

3. $c \wedge((a \wedge b) \rightarrow a)=c$;

4. $a \leq b$ se, e somente se, $a \rightarrow b=1$;

5. $a=(1 \rightarrow a)$;

6. $a \rightarrow(b \wedge c)=(a \rightarrow b) \wedge(a \rightarrow c)$.

Proposição A.2.3. Toda álgebra de Heyting é um reticulado distributivo.

Definição A.2.4. Sejam $(H, \leq)$ uma álgebra de Heyting e $a \in H$. O pseudo-complemento de $a$ é definido como $\neg a:=a \rightarrow 0$.

Proposição A.2.5. Sejam $(H, \leq)$ uma álgebra de Heyting e $a, b \in H$. Então,

\footnotetext{
${ }^{2}$ Aliás, bastaria possuir menor elemento, pois $1=a \rightarrow a$, para qualquer $a \in H$.
} 
1. $\neg 0=1 e \neg 1=0$;

2. se $a \leq b$, então $\neg b \leq \neg a$;

3. $\neg a=\neg \neg \neg a$;

4. $\neg(a \vee b)=\neg a \wedge \neg b$;

5. $(\neg a \vee b) \leq(a \rightarrow b)$

6. $a \leq \neg \neg a$;

7. $\neg \neg(a \wedge b)=(\neg \neg a \wedge \neg \neg b)$;

8. $\neg \neg(a \rightarrow b)=(\neg \neg a \rightarrow \neg \neg b)$.

Tendo definido o pseudo-complemento, pode-se mostrar que álgebras de Heyting servem de modelo algébrico para a lógica proposicional intuicionista. Mais especificamente, podemos definir uma relação de ordem no conjunto das fórmulas dessa lógica fazendo

$$
\varphi \leq \psi \quad \text { se, e somente se, } \quad \vdash \varphi \rightarrow \psi
$$

de forma que toda álgebra de Heyting satisfaz os axiomas do cálculo proposicional intuicionista:

Proposição A.2.6. Sejam $(H, \leq)$ uma álgebra de Heyting e a,b,c $\in$ H. Então:

1. $a \leq(b \rightarrow a)$;

2. $(a \rightarrow(b \rightarrow c)) \leq((a \rightarrow b) \rightarrow(a \rightarrow c))$;

3. $a \leq(b \rightarrow(a \wedge b))$;

4. $(a \wedge b) \leq a$;

5. $(a \wedge b) \leq b$;

6. $a \leq(a \vee b)$;

7. $b \leq(a \vee b)$;

8. $(a \rightarrow c) \leq((b \rightarrow c) \rightarrow((a \wedge b) \rightarrow c))$

9. $(a \rightarrow b) \leq((a \rightarrow \neg b) \rightarrow \neg a)$;

10. $\neg a \leq(a \rightarrow b)$; 
11. Modus ponens: $a \wedge(a \rightarrow b) \leq b$.

Para finalizar essa seção, definamos elementos regulares, que fornecerão uma ligação entre álgebras de Heyting e álgebras de Boole.

Definição A.2.7. Sejam $(H, \leq)$ uma álgebra de Heyting e $a \in H$. Dizemos que $a$ é regular se $a=\neg \neg a$. O conjunto de elementos regulares de $H$ é denotado por $\operatorname{Reg}(H)$.

\section{A.3 Álgebras de Boole}

Definição A.3.1. Uma álgebra de Boole é um reticulado distributivo limitado $(B, \leq)$ onde todo elemento possui complemento, isto é, para todo $a \in B$, existe um $b \in B$ (que será único por distributividade) tal que $a \wedge b=0$ e $a \vee b=1$.

Esse único $b$ será chamado de complemento de $a$ e será denotado por $\neg a$. A coincidência com a notação de pseudo-complementos é intencional:

Definição A.3.2. Sejam $(B, \leq)$ uma álgebra de Boole e $a, b \in B$. Então, definimos o elemento $a \rightarrow b:=\neg a \vee b$.

Proposição A.3.3. Seja $(B, \leq)$ uma álgebra de Boole. Então, para todos $a, b, c \in B, c \leq(a \rightarrow b)$ se, e somente se, $a \wedge c \leq b$. Como consequência disso, também vale $a \leq b$ se, e somente se, $a \rightarrow b=1$, e o complemento de a satisfaz $\neg a=(a \rightarrow 0)$.

Dessa forma, toda álgebra de Boole é uma álgebra de Heyting e o complemento é exatamente o pseudo-complemento.

Lema A.3.4. Seja $(H, \leq)$ uma álgebra de Heyting. Então, Reg $(H)$ é uma álgebra de Boole (onde o supremo $\vee^{\prime}$ de $\operatorname{Reg}(H)$ é obtido do supremo $\vee$ de $H$ por $a \vee^{\prime} b=\neg \neg(a \vee b)$ ).

Proposição A.3.5. Seja $(H, \leq)$ uma álgebra de Heyting. Então, são equivalentes:

1. $(H, \leq)$ é uma álgebra de Boole;

2. para todo $a \in H, a \vee \neg a=1$;

3. para todo $a \in H, a=\neg \neg a$. 
Essa proposição mostra, assim, que álgebras de Boole servem de modelo algébrico para o cálculo proposicional clássico.

Por fim, listemos algumas propriedades das operações em álgebras de Boole.

Proposição A.3.6. Sejam $(B, \leq)$ um álgebra de Boole, $a, b \in B$ e $S \subseteq B$. Então,

1. $a \leq b$ se, e somente se, $\neg b \leq \neg a$;

2. $\neg(a \wedge b)=\neg a \vee \neg b e \neg(a \vee b)=\neg a \wedge \neg b$;

3. se $\bigvee S$ existe (em B), então $\bigwedge_{s \in S} \neg s$ e $\bigvee_{s \in S}(a \wedge s)$ existem e satisfazem:

$$
\neg \bigvee S=\bigwedge_{s \in S} \neg s \quad e \quad a \wedge \bigvee S=\bigvee_{s \in S}(a \wedge s)
$$

4. se $\bigwedge S$ existe (em B), então $\bigvee_{s \in S} \neg s$ e $\bigwedge_{s \in S}(a \vee s)$ existem e satisfazem:

$$
\neg \bigwedge S=\bigvee_{s \in S} \neg s \quad e \quad a \vee \bigwedge S=\bigwedge_{s \in S}(a \vee s)
$$




\section{Bibliografia}

[ACM19] José G. Alvim, Arthur F. S. Cahali e Hugo L. Mariano. "Localic topoi, Heyting-valued models and induced morphisms". Trabalho em andamento. 2019.

[AGV72] Michael Artin, Alexander Grothendieck e Jean-Louis Verdier. Theorie de Topos et Cohomologie Etale des Schemas. Lecture Notes in Mathematics, vol. 269. Heidelberg, Alemanha: Springer-Verlag, 1972.

[AH16] Jean-Martin Albert e Bradd Hart. "Metric logical categories and conceptual completeness for first order continuous logic". Em: arXiv e-prints (2016). arXiv: 1607.03068v1.

[Bel05] John L. Bell. Set theory. Boolean-valued models and independence proofs. $3^{\mathrm{a}}$ ed. Oxford Logic Guides, vol. 47. Oxford, Reino Unido: Clarendon Press, 2005.

[Bel14] John L. Bell. Intuitionistic Set theory. Studies in Logic, vol. 50. Londres, Reino Unido: College Publications, 2014.

[Bel88] John L. Bell. Toposes and local set theories. an introduction. Oxford Logic Guides, vol. 14. Oxford, Reino Unido: Clarendon Press, 1988.

[BL67] Garret Birkhoff e Saunders Mac Lane. Algebra. $1^{\text {a }}$ ed. Nova Iorque, Estados Unidos: Macmillan, 1967.

[BM06] Bernhard Banaschewski e Christopher J. Mulvey. "A globalisation of the Gelfand duality theorem". Em: Annals of Pure and Applied Logic 137 (2006), pp. 62-103. DOI: https://doi.org/10.1016/j.apal.2005.05.018.

[Bor08a] Francis Borceux. Handbook of Categorical Algebra. Vol. 1. Encyclopedia of mathematics and its applications, vol. 50. Cambridge, Reino Unido: Cambridge University Press, 2008.

[Bor08b] Francis Borceux. Handbook of Categorical Algebra. Vol. 2. Encyclopedia of mathematics and its applications, vol. 51. Cambridge, Reino Unido: Cambridge University Press, 2008. 
[Bor08c] Francis Borceux. Handbook of Categorical Algebra. Vol. 3. Encyclopedia of mathematics and its applications, vol. 52. Cambridge, Reino Unido: Cambridge University Press, 2008.

[BS89] Andreas R. Blass e Andre Scedrov. Freyd's Models for the Independence of the Axiom of Choice. Memoirs of the American Mathematical Society, vol. 79, número 404. Providence, Estados Unidos: American Mathematical Society, 1989.

[BW85] Michael Barr e Charles Wells. Toposes, Triples and Theories. Grundlehren der mathematischen Wissenschaften, vol. 278. Nova Iorque, Estados Unidos: Springer-Verlag, 1985.

[EM01] Mehdi Ebrahimi e Mojgan Mahmoudi. "The category of M-sets". Em: Italian Journal of Pure and Applied Mathematics 9 (2001), pp. 123-132.

[Gol79] Robert I. Goldblatt. Topoi. The categorial analysis of logic. Studies in Logic and the Foundations of Mathematics, vol. 98. Amsterdã, Países Baixos: North-Holland Publishing Company, 1979.

[Gra79a] John W. Gray. "Fragments of the history of sheaf theory". Em: Applications of Sheaves. Ed. por Michael P. Fourman, Christopher J. Mulvey e Dana S. Scott. Lecture Notes in Mathematics, vol. 753. Heidelberg: Springer-Verlag, 1979. Cap. 1, pp. 1-79.

[Gra79b] Robin J. Grayson. "Heyting-valued models for intuitionistic set theory". Em: Applications of Sheaves. Ed. por Michael P. Fourman, Christopher J. Mulvey e Dana S. Scott. Lecture Notes in Mathematics, vol. 753. Heidelberg, Alemanha: Springer-Verlag, 1979. Cap. 14, pp. 402-414.

[Gro57] Alexander Grothendieck. "Sur quelques points d'algèbre homologique, I". Em: Tohoku Mathematical Journal 9 (1957), pp. 119-221. DOI: https://doi.org/10.2748/tmj/ 1178244839.

[Gui13] Fiorella Guichardaz. "Limits of Boolean algebras and Boolean valued models". Diss. de mestrado. Universitá degli studi di Torino, 2013.

[Jec03] Thomas J. Jech. Set Theory. The third millennium edition, revised and expanded. Springer Monographs in Mathematics. Heidelberg, Alemanha: Springer-Verlag, 2003.

[Joh02a] Peter T. Johnstone. Sketches of an Elephant. A Topos Theory Compendium. Vol. 1. Oxford Logic Guides, vol. 43. Nova Iorque, Estados Unidos: Oxford University Press, 2002. 
[Joh02b] Peter T. Johnstone. Sketches of an Elephant. A Topos Theory Compendium. Vol. 2. Oxford Logic Guides, vol. 44. Nova Iorque, Estados Unidos: Oxford University Press, 2002 .

[Lan98] Saunders Mac Lane. Categories for the Working Mathematician. $2^{\mathrm{a}}$ ed. Graduate texts in mathematics, vol. 5. Nova Iorque, Estados Unidos: Springer-Verlag, 1998.

[LM94] Saunders Mac Lane e Ieke Moerdijk. Sheaves in Geometry and Logic. A First Introduction to Topos Theory. $2^{\mathrm{a}}$ ed. Universitext. Nova Iorque, Estados Unidos: SpringerVerlag, 1994.

[LS86] Joachin Lambek e Philip J. Scott. Introduction to higher order categorical logic. Cambridge studies in advanced mathematics, vol. 7. Cambridge, Reino Unido: Cambridge University Press, 1986.

[Mc195] Colin Mclarty. Elementary Categories, Elementary Toposes. Oxford Logic Guides, vol. 21. Oxford, Reino Unido: Clarendon Press, 1995.

[Mir06] Francisco Miraglia. An Introduction to Partially Ordered Structures and Sheaves. Milão, Itália: Polimetrica, 2006.

[MR77] Michael Makkai e Gonzalo E. Reyes. First Order Categorical Logic. Model-theoretical Methods in the Theory of Topoi and Related Categories. Lecture Notes in Mathematics, vol. 611. Berlim, Alemanha: Springer-Verlag, 1977.

[Mul80] Christopher J. Mulvey. "Banach Sheaves". Em: Journal of Pure and Applied Algebra 17 (1980), pp. 69-83. DOI: https://doi.org/10.1016/0022-4049(80)90023-7.

[Rut65] Daniel E. Rutherford. Introduction to Lattice Theory. University Mathematical Monographs, vol. 2. Edinburgo, Reino Unido: Oliver \& Boyd, 1965.

[Shu10] Michael A. Shulman. "Stack semantics and the comparison of material and structural set theories". Em: arXiv e-prints (2010). arXiv: 1004.3802v1.

[Ten75] Barry R. Tennison. Sheaf Theory. London Mathematical Society Lecture Note Series, vol. 20. Cambridge, Reino Unido: Cambridge University Press, 1975. 


\section{Lista de símbolos}

$(-)^{b}, 68$

$-\alpha, 117$

$F \Rightarrow G, 15$

$F \downarrow b, 37$

$F(-)(b), 16$

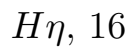

$H$-Set, 154

$J_{\text {cov }}, 60$

$L \dashv R, 33$

Mono(a), 29

$\operatorname{Sub}(-), 33$

$\operatorname{Sub}(a), 29$

$V^{(H)}=\sigma, 152$

$\left[\gamma_{1}, \gamma_{2}\right], 22$

$\Delta_{A}, 126$

$\Leftrightarrow: \Omega \times \Omega \rightarrow \Omega, 113$

$\Sigma_{c}, 38$

$\Vdash_{w}, 162$

$\Vdash \vdash, 161$

$V^{(H)}, 151$

$\alpha$ ก $\beta, 104$

$\alpha \uplus \beta, 107$

$\alpha \Rightarrow \beta, 113$

$\perp: \mathbf{1} \longmapsto \Omega, 98$

$\chi_{m}, 73$

$\eta K, 16$

$\exists_{f}, 121$

$\forall_{f}, 121$

$\left\langle\gamma_{1}, \gamma_{2}\right\rangle, 21$

$\lim _{c \in \mathcal{C}_{0}} F(c), 25$

$\llbracket \sigma \rrbracket^{H}, 151$

$\llbracket \varphi \rrbracket, 128$

0, 15
1, 15

Loc, 48

Set, 7

Set $^{(H)}, 153$

$\operatorname{Sh}(X), 45$

Top, 7

cH-Set, 155

$\mathcal{A} \simeq \mathcal{B}, 17$

$\mathcal{B}^{\mathcal{A}}, 16$

$\mathcal{C} \downarrow c, 38$

$\mathcal{C}(-,-), 12$

$\mathcal{C}(a, b), 7$

$\mathcal{C}(c,-), 12$

$\mathcal{C}^{o p}, 10$

$\mathcal{E}=\varphi, 126$

$\mathcal{L}_{\mathcal{E}}, 125$

$\mathcal{P}(a), 77$

$\mathrm{I}_{c}, 15$

$\mathrm{O}_{c}, 15$

$\neg: \Omega \rightarrow \Omega, 117$

$\ominus_{A}, 126$

$\bar{f}, 170$

$\downarrow a, 49$

$\tilde{f}, 165$

$\top_{a}, 107$

$\ulcorner t\urcorner, 125$

$\varrho(x), 151$

$\vee: \Omega \times \Omega \rightarrow \Omega, 107$

$\wedge: \Omega \times \Omega \rightarrow \Omega, 104$

$\{*\}, 8$

$a \cong b, 8$

$a \in_{u} A, 137$

$a \longmapsto b, 13$ 
$a \rightarrow b, 13$

$b \downarrow F, 37$

$c \downarrow \mathcal{C}, 38$

$c^{\star}, 39$

$e v_{c}, 68$

$\exp (h), 68$

$f+g, 22$

$f \cap g, 30$

$f \cup g, 31$

$f \preceq f^{\prime}, 29$

$f \sim f^{\prime}, 29$

$f \times g, 21$

$f^{\star}, 41$

$f^{\triangleright}, 14$

$f^{*} S, 58$

$f^{o p}, 11$

$i d_{a}, 7$

$i m(f), 14$

$s \uparrow_{V}^{U}, 45$

$t\left(a_{1}, \ldots, a_{n}\right) \in_{u} A, 138$

$u \Vdash \varphi\left(a_{1}, \ldots, a_{n}\right), 138$ 


\section{Índice remissivo}

$H$-set, 153

- completo, 155

Adjunção, 33

Categoria, 6

- balanceada, 74

- bem potenciada, 33

- cartesianamente fechada, 68

- cocompleta, 25

- completa, 25

- coslice, 38

- de funtores, 16

- finitamente cocompleta, 25

- finitamente completa, 25

- localmente pequena, 8

- oposta, 10

- pequena, 8

- produto (binário), 12

- slice, 38

Classificador de subobjetos, 73

Cobertura (de um estágio), 137

Cobertura hereditária, 56

Cocone, 19

Coequalizador, 24

Cokernel par, 26

Colimite, 20

Cone, 19

Conjunção, 104

Coproduto, 22

Counidade (adjunção), 34

Crivo, 58

Diagrama comutativo, 7

Disjunção, 107

Dualidade (princípio da), 11

Epi, 13

$$
\text { - regular, } 26
$$

Epimórfica (família), 21

Equalizador, 24

Equivalência de categorias, 17

Estágio (de uma família geradora), 137

Fatoração epi-mono, 14

Feixe (funtorial)

- sobre um espaço topológico, 45

- sobre um locale, 49

- sobre um sítio, 61

Feixe geométrico
- sobre um espaço topológico, 44

- sobre um locale, 49

Fibra

- de um prefeixe, 46

- de uma função, 44

Flecha característica, 73

Funtor, 8

- de Yoneda, 18

- fiel, 10

- hom, 11

- inclusão, 10

- monádico, 83

- oposto, 11

- pleno, 10

- produto, 12

- representável, 16

bifuntor, 12

preserva limites, 25

Funtor lógico, 80

Gerador, 14

Homeomorfismo local

- de espaços topológicos, 44

- de locales, 49

Implicação, 113

Injeção canônica, 22

Intersecção (de subobjetos), 30

Isomorfismo (ou iso), 8

Isomorfismo de categorias, 10

Isomorfismo natural, 15

Kernel par, 26

Lema de Yoneda, 17

Lema do pullback, 26

Limite, 19

Linguagem de Mitchell-Bénabou, 123 - de um topos, 125

Locale, 47

Mono, 13

$$
\text { - regular, } 26
$$

Morfismo de $H$-sets, 154

Morfismo de locales, 48

Morfismo geométrico

- entre topoi de Grothendieck, 64

- entre topoi elementares, 81

Negação, 117 
Objeto inicial, 14

Objeto números naturais, 41

Objeto partes, 77

Objeto terminal, 14

Prefeixe

- separado, 61

- sobre um espaço topológico, 45

- sobre um locale, 49

- sobre um sítio, 60

Pretopologia de Grothendieck, 58

Produto, 21

Projeção, 21

Pullback, 23

Pullback (de um crivo), 58

Pushout, 23

Relação de forcing

- forte (ou de Cohen), 163

- fraca, 162

- intuicionista, 161

Retração, 14

Sítio, 60

Seção, 14

- contínua, 44

Subcategoria, 10

- plena, 10

Subconjunto hereditário, 56

Subfuntor, 30

Subobjeto, 29

Subprefeixe fechado, 51

Teorema especial do funtor adjunto, 36

Teorema geral do funtor adjunto, 36

Testemunha, 165

Topologia de Grothendieck, 59

- canônica, 62

- subcanônica, 62

Topos

- booleano, 99

- de Grothendieck, 63

- elementar, 77

Transformação natural, 15

Transposta exponencial, 68

União (de subobjetos), 30

Unidade (adjunção), 34

Validade de fórmulas em $\mathcal{L}_{\mathcal{E}}, 126$ 